\title{
Copyright
}

by

Kristy Joy Kormondy

2017 
The Dissertation Committee for Kristy Joy Kormondy Certifies that this is the approved version of the following dissertation:

\section{Oxide Materials at the Two-Dimensional Limit}

\section{Committee:}

\begin{tabular}{l}
\hline Alexander A. Demkov, Supervisor \\
\hline Keji Lai \\
\hline Alejandro L. De Lozanne \\
\hline Maxim Tsoi \\
\hline John G. Ekerdt
\end{tabular}




\title{
Oxide Materials at the Two-Dimensional Limit
}

$$
\text { by }
$$

Kristy Joy Kormondy, B.S.

\author{
Dissertation \\ Presented to the Faculty of the Graduate School of \\ The University of Texas at Austin \\ in Partial Fulfillment \\ of the Requirements \\ for the Degree of \\ Doctor of Philosophy
}

The University of Texas at Austin

May 2017 


\section{Dedication}

For my sisters Michelle and Charlene. 


\section{Acknowledgements}

I am grateful to my advisor, Professor Alex Demkov, for his guidance and support. I would also like to thank Jean Fompeyrine and Stefan Abel, my supervisors at IBM ZRL.

I have learned much thanks to the members of the Demkov Group (Agham, Chungwei, Richard, Chandrima, Lukas, Alex S., Hosung, Miri, Andy, Kurt, Patrick, Donghan, Lingyuan, Elliott, Ali, Tobi, Wei, and Jackie), Professor John Ekerdt and the members of his group (Martin, Thong, Ed, Bryce, Shen, and Annie), and the many talented people at IBM (Heike, Chiara, Marilyne, Daniele, Heinz, Marta, Steffen, Lukas, Vladimir, Veeresh, Emanuele, Felix, Florian, Youri, and Mateusz).

This work would not have been possible without the Air Force Office of Scientific Research (AFOSR) grant FA9550-12-10494 and the National Science Foundation grant IRES- 1358111. 


\title{
Oxide Materials at the Two-Dimensional Limit
}

\author{
Kristy Joy Kormondy, Ph.D. \\ The University of Texas at Austin, 2017
}

Supervisor: Alexander A. Demkov

Emergent phenomena in transition metal oxide films are receiving considerable attention with the development of techniques for the preparation of well-controlled oxide surfaces. On the macroscopic scale, such display novel physics phenomena including superconductivity, magnetism, ferroelectricity, and more. On the nanometer scale, the properties of epitaxial interfaces are further impacted by strain, band alignment, and crystal imperfections that may affect the long-range as well as the short-range order. Furthermore, symmetry lowering at the interface creates entirely new environments that are not accessible in the bulk environment. Thus, thin-film oxide materials are increasingly important in many applications. My work focuses on epitaxial oxides of the perovskite, spinel, and rocksalt structure and covers two main phenomena: (1) the twodimensional electron gas at epitaxial oxide interfaces, and (2) thin epitaxial electro-optic oxides.

Because polar oxides are of prominent interest as a mechanism for the formation of the two-dimensional electron gas, I start with a study of polar semiconductor $\mathrm{Co}_{3} \mathrm{O}_{4}$. Ellipsometry reveals a direct band gap of $0.75 \mathrm{eV}$, and magnetic measurements show the signature of antiferromagnetic ordering at $49 \mathrm{~K}$, higher than the typical bulk value. Next, I look closer at the role of defects by studying the highly conducting layer at the 
crystalline $\gamma$-alumina/ $/ \mathrm{SrTiO}_{3}$ (STO) interface which is attributed to oxygen vacancies. Annealing in oxygen is found to reduce the carrier density and turn a conductive sample into an insulator. Building upon these results, I show that even at room temperature, outdiffusion of oxygen from $\mathrm{SrTiO}_{3}$ during epitaxy of highly spin-split semiconductor $\mathrm{EuO}$ epitaxy creates a highly conductive layer of oxygen vacancies on the $\mathrm{SrTiO}_{3}$ side of the interface. The films are ferromagnetic with a Curie temperature of $70 \mathrm{~K}$ and display giant magnetoresistance below the transition temperature. Leveraging this approach offers an as-yet unexplored route to seamlessly integrate ferromagnetism and the oxide twodimensional electron gas for the development of novel nano-oxide spintronic devices.

The large effective Pockels coefficient for high-quality epitaxial BTO films on Si distinguishes $\mathrm{BaTiO}_{3}$ as a highly promising material for integrated silicon nanophotonics. However, the linear electro-optic effect in $\mathrm{BaTiO}_{3}$ thin films determined in previous experiments clearly shows deteriorated properties compared to bulk BTO crystals. First, I study $\mathrm{BaTiO}_{3}$ films of varied thickness in order to quantify the Pockels coefficient with respect to crystalline orientation. As a next step, I report on the strong dependence of the Pockels effect in $\mathrm{BaTiO}_{3}$ thin films on their microstructure, and provide guidelines on how to engineer thin films with strong electro-optic response. The $25 \times$ enhancement of the Pockels coefficient indicates a promising route to increase the performance of nonlinear oxides in the two-dimensional limit for the development of novel hybrid silicon photonic platform. 


\section{Table of Contents}

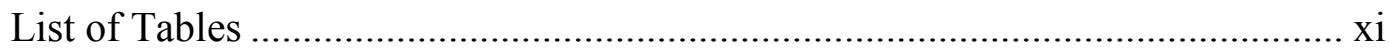

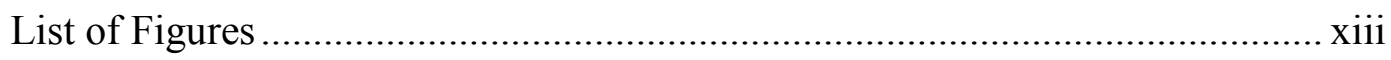

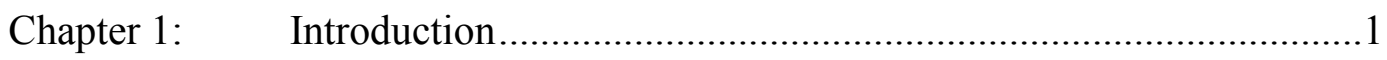

1.1. New Opportunities with Oxide Interfaces ………………………….......

1.2. Two-Dimensional Electron Gas at Oxide Interfaces ..............................2

1.2.1. Oxygen Vacancies in LAO/STO ........................................

1.3. Films of Integrated Nonlinear Oxides..................................................

1.4. Objective and Overview of Chapters ..................................................11

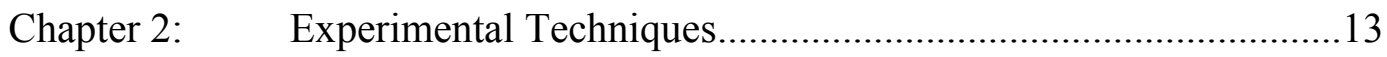

2.1. Molecular Beam Epitaxy ..............................................................13

2.2. X-ray Photoelectron Spectroscopy ……………………………….....16

2.3. Free-space Electro-optic Characterization ............................................17

Chapter 3: $\quad$ Epitaxy of polar semiconductor $\mathrm{Co}_{3} \mathrm{O}_{4}(110) \ldots \ldots \ldots \ldots \ldots \ldots \ldots \ldots \ldots . . . .21$

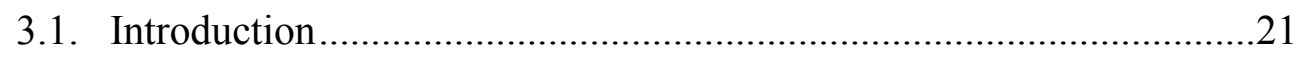

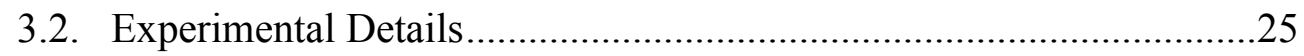

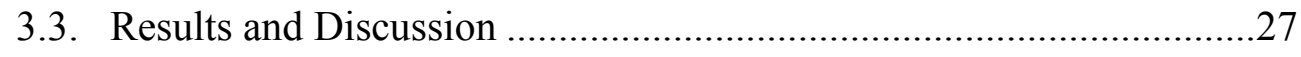

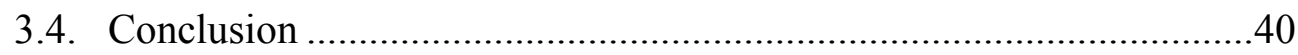

Chapter 4: Quasi-two-dimensional electron gas at the epitaxial alumina/ $\mathrm{SrTiO}_{3}$ interface 42

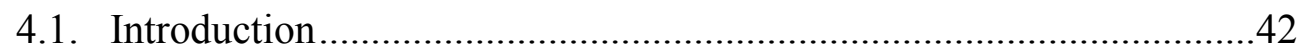

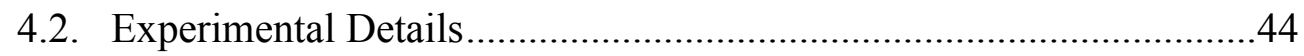

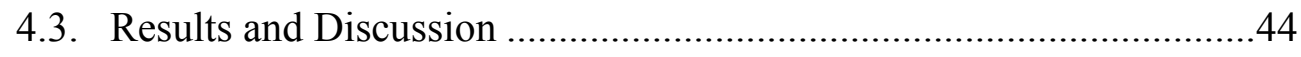

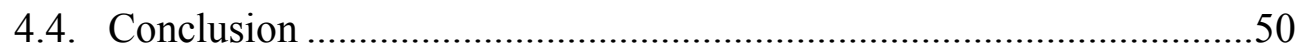

Chapter 5: $\quad$ Positive linear magnetoresistance in the two-dimensional electron

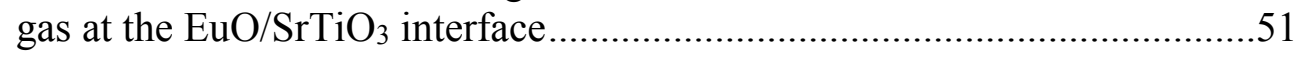

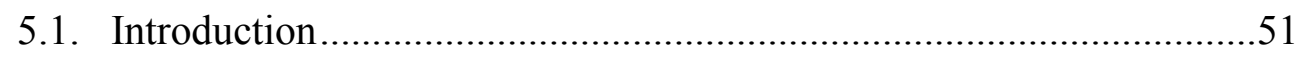

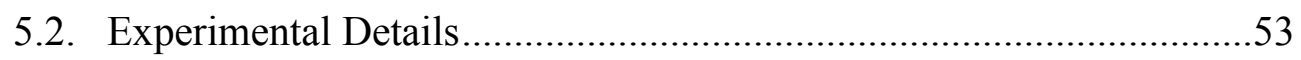


5.2.1. Film deposition ............................................................. 53

5.2.2. Sample Characterization ........................................................

5.2.3. Density functional theory.................................................

5.2.4. Soft-X-ray ARPES experiments ........................................55

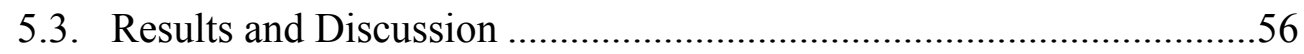

5.3.1. Sample Preparation and Characterization ...........................56

5.3.2. Electrical Characterization.................................................58

5.3.3. First-principles Calculations …………………………......60

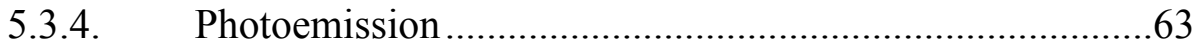

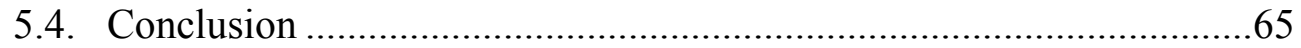

Chapter 6: $\quad$ Analysis of the Pockels effect in ferroelectric barium titanate thin

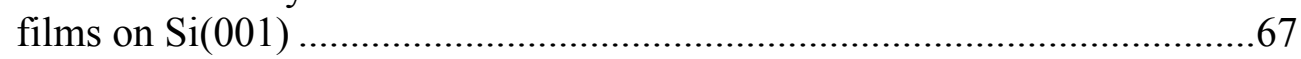

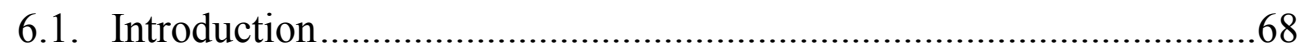

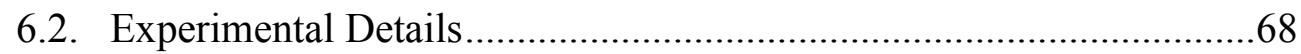

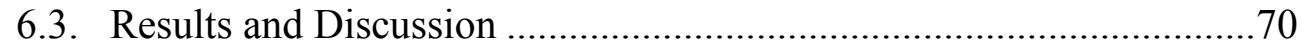

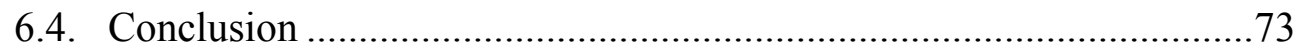

Chapter 7: $\quad$ Microstructure and ferroelectricity of $\mathrm{BaTiO}_{3} / \mathrm{Si}$ for integrated photonics 74

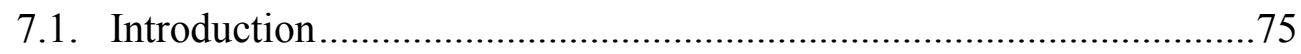

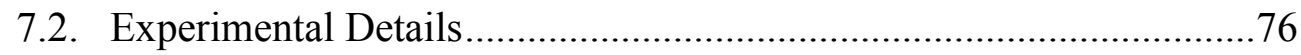

7.2.1. Sample preparation ..........................................................

7.2.2. Structural characterization ..................................................

7.2.3. Electro-optic characterization ………………………........

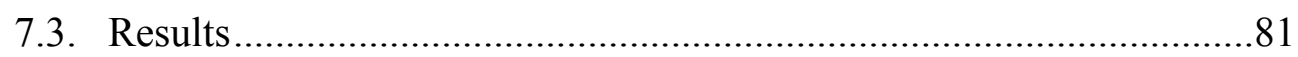

7.3.1. Structural Characterization ................................................ 81

7.3.2. Electro-optic Characterization ...........................................87

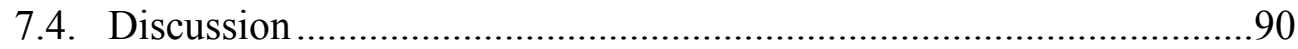

7.4.1. Electric Field Distribution..................................................91

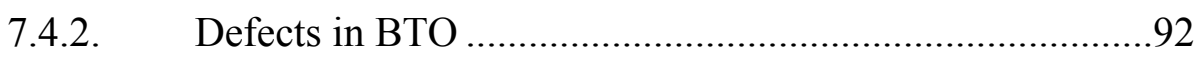

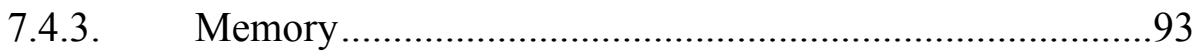




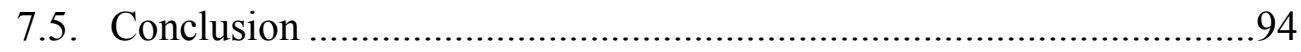

Chapter 8: Summary and Future Work..........................................................96

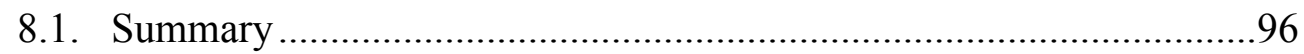

8.1.1. Two-Dimensional Electron Gas at Oxide Interfaces .........96

8.1.2. Films of Integrated Nonlinear Oxides.................................98

8.2. Recommendations for Future Work.....................................................99

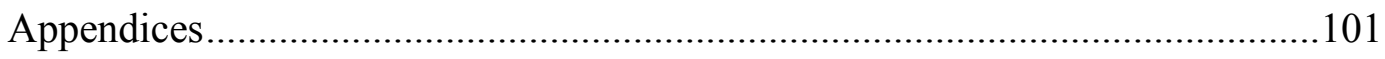

Appendix A: Supplement to Chapter 4:Positive linear magnetoresistance in the two-dimensional electron gas at the $\mathrm{EuO} / \mathrm{SrTiO}_{3}$ interface ................101

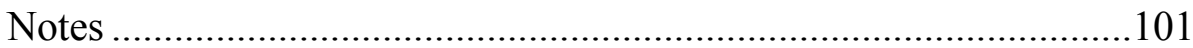

Note 1: Valence Band Offsets....................................................101

Note 2: Positive MR Derivation ..............................................102

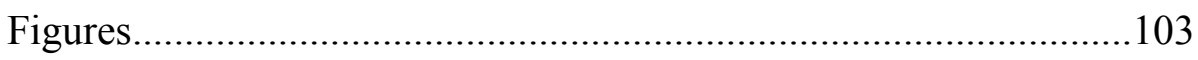

Appendix B: Supplement to Chapter 7:Microstructure and ferroelectricity of $\mathrm{BaTiO}_{3} / \mathrm{Si}$ for integrated photonics.................................................113

Note: Simulation Details...............................................................118

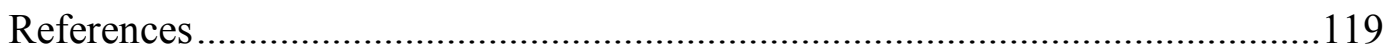




\section{List of Tables}

Table 2.1: Evaporation rate calibration parameters for metals used in this dissertation.

Table 3.1: XPS core levels for valence band offset calculation. 32

Table 6.1: Pockels coefficients for samples grown under various growth conditions. .73

Table 7.1: Summary of film microstructure parameters. From ellipsometry, we extract the refractive index $n$ of the BTO matrix, porosity volume fraction $p$ and thickness of the BTO layer $t$. Error bars of porosity correspond to a BTO thickness variation of $5 \%$ (details in the text). From STEM, $e d r$ is the average equivalent disc radius of the grains highlighted in Figure 7.2.

Table 7.2: Results from XRD analysis of $c$ and $a$ lattice parameters for BTO P4mm symmetry. Tetragonality $\gamma=c / a-1$. Error bars correspond to uncertainty of fit parameters. 86

Table 7.3: Summary of best-fit Pockels tensor elements $r_{42}$ and $r_{33}$ for each BTO sample. Effective Pockels coefficient $r_{\text {eff }}$ and $c$-axis coefficient $r_{\mathrm{c}}$ are calculated according to Eq. (7.2). Error bars for $r_{42} r_{13}$ were estimated by repeating the fitting procedure for $n_{\mathrm{b}}=0.02$ and 0.04 . Error bars for $r_{\mathrm{eff}}$ and $r_{\mathrm{c}}$ correspond to pad-to-pad variation determined from multiple measurements of the $\theta_{\mathrm{E}}=45^{\circ}$ orientation for each sample. .88

Table 7.4: Summary of parameters related to ferroelectricity from electro-optic measurements, remanence ratio $\delta_{r}^{\prime} / \delta^{\prime}{ }_{s}$ and coercive field $E_{\mathrm{c}}$. Error bars correspond to uncertainty of fit parameters. 
Table B.1: Summary of additional film structural and electrical parameters. From the atomic force microscopy images in Figure B.2, the RMS surface roughness can be extracted for all samples. The FWHM of the rocking curves in Figure B.3(b) were also calculated. The leakage current at a DC bias of $35 \mathrm{~V}$ is shown for all samples......................................117

Table B.2: Parameters used for simulating $\delta^{\prime}$ as a function of $\theta_{\mathrm{E}}$ (Figure B.4). *fit

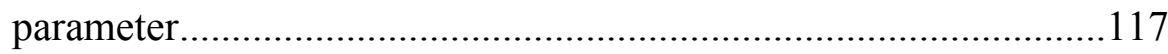

Table B.3: Parameters used for COMSOL simulations of correction factor $c_{\mathrm{p}}$ for porous structures shown in Figure B.6. Variables included distance between electrodes $d$, lateral periodicity $w$, electric potential drop $\varphi$, porosity $p$, dielectric constant $\varepsilon_{\mathrm{BTO}}$, and pore cross-section shape (circle, square, or slab). 


\section{List of Figures}

Figure 1.1: Highly conductive interfaces can be formed between two insulating oxides due to (a) electronic reconstruction, (b) oxygen vacancies, and (c) stoichiometry deviations. 2

Figure 1.2: (a) Illustration of electrostatic model for the polar catastrophe. (b) and (c) illustrate critical thickness for electronic reconstruction. .4

Figure 1.3: Tetragonal $\mathrm{BaTiO}_{3}$ has a center of inversion (top), while ferroelectric $\mathrm{BaTiO}_{3}$ is non-centrosymmetric (bottom).

Figure 1.4: Electro-optical Pockels coefficients $r$ for various materials [73-75].10

Figure 1.5: Overview of dissertation chapters. 12

Figure 2.1: Schematic (left) and picture (right) of customized oxide molecular beam epitaxy chamber in the Materials Physics Lab at UT Austin. Labels indicate include components in the top-half (cooling panel, substrate, RHEED gun, phosphor screen, thickness monitor) and bottom-half (cryopump, effusion cells and shutters, electron beam gun, $\mathrm{O}_{2}$ gas) of the chamber. 13

Figure 2.2: Left: Low-temperature effusion cell. A knife-edge covered by a copper gasket in the bottom of the image is crucial to maintain UHV when the cell is mounted on the chamber pictured in Figure 2.1. Right: PBN crucible filled with metal in specialized cold-lipped effusion cell shows structural damage after repeated temperature cycling. .14 
Figure 2.3: RHEED intensity oscillations for a fixed azimuth during STO deposition on $\mathrm{Si}(001)$. Each individual oscillation indicates the completion of one atomic layer of STO. From a sinusoidal fit to the data (red), the directly measured time for deposition of one atomic layer is approximately 1 minute and 4 seconds for this sample....................................... 16

Figure 2.4: XPS analysis chamber in the Materials Physics Lab at UT Austin.....16 Figure 2.5: Ti $2 p$ (a) and valence band (b) spectrum of $\mathrm{Nb}$-doped $\mathrm{SrTiO}_{3}(100)$ substrate after a water boiling process and vacuum annealing [84]. 17

Figure 2.6: (a) Picture and (b) schematic of Sénarmont setup components. A halfwave plate is used to set the incident linear polarization. After passing through the sample, the light becomes elliptically polarized, which is transformed into linearly polarized light after passing a quarter-wave plate. Finally, a Glan-laser prism is used to determine the orientation of the transmitted polarization. (c) Corresponding states of the polarization along the path of the laser beam are visualized as the ellipse traced out by the light's electric field vector with (red) and without (blue) an external electric field. After transmission through the sample and quarter-wave plate, the polarization is rotated by angle $\delta$. (d) Detector for recording changes in transmission. 18

Figure 3.1: At room temperature, $\mathrm{Co}_{3} \mathrm{O}_{4}$ assumes the normal spinel structure. Based on the crystal field splitting, the $\mathrm{Co}^{2+}$ ions (tetrahedral, light blue) carry magnetic moment, while the $\mathrm{Co}^{3+}$ (octahedral, dark blue) ions are nonmagnetic. .22 
Figure 3.2: Side view: the (110) direction of $\mathrm{Co}_{3} \mathrm{O}_{4}$ is characterized by a 4-repeat period over a depth of $5.7 \AA$. The alternating planes are shown from a top view. The type A plane is comprised of units of $\mathrm{Co}^{3+}{ }_{2} \mathrm{Co}^{2+}{ }_{2} \mathrm{O}_{4}$ with a formal charge of +2 , while the type $\mathrm{B}$ planes include $\mathrm{Co}^{3+}{ }_{2} \mathrm{O}_{4}$ resulting in a charge of -2 .24

Figure 3.3: RHEED patterns along the [110] azimuth at grazing incidence for (a) the $\mathrm{MgAl}_{2} \mathrm{O}_{4}$ substrate, (b) $\sim 13$ - $\AA$-thick $\mathrm{Co}_{3} \mathrm{O}_{4}$ film, (c) $\sim 24-\AA$-thick film, (d) 125 - $\AA$-thick film. The most stable surface of the $\mathrm{MgAl}_{2} \mathrm{O}_{4}$ substrate is the A-type, which contains both $\mathrm{Mg}$ and $\mathrm{Al}$ atoms. The $\mathrm{Co}_{3} \mathrm{O}_{4}$ film follows the termination of the substrate to preserve charge neutrality, as is common in polar materials. The surface develops roughness at thickness starting between 10-20 A, causing the observed broadening of the RHEED streaks. The surface again becomes smooth as growth continues, returning to substrate quality around $100 \AA$ thickness. ...28

Figure 3.4: AFM measurements for: (a) bare $\mathrm{MgAl}_{2} \mathrm{O}_{4}$ substrate, followed by $\mathrm{Co}_{3} \mathrm{O}_{4}$ samples of thickness (b) $13 \AA$, (c) $24 \AA$, and (d) $125 \AA$. The RMS roughness measured was $1.52 \AA, 1.26 \AA, 5.26 \AA$, and $1.04 \AA$, respectively. 
Figure 3.5: (a) Rocking curve data and analysis of the (440) reflection of $240 \AA$ film $\left(\mathrm{Co}_{3} \mathrm{O}_{4}\right.$ on $\left.\mathrm{MgAl}_{2} \mathrm{O}_{4}\right)$. Experimental data points are shown by open circles, and a sum of two Voigt functions has been fitted to the data. (b) X-ray reflectivity for the same film. The drawn (red) line is a simulation. Oscillations due to the difference in density between the substrate and film persist undamped to high angles, implying a smooth surface and abrupt interface. (c) High-resolution cross-sectional transmission electron micrograph confirms the abrupt interface between the crystalline substrate and film. 30

Figure 3.6: XPS spectra. (a) Survey scan of representative 250 - $\AA$-thick $\mathrm{Co}_{3} \mathrm{O}_{4}$ film shows a spectrum dominated by cobalt and oxygen. (b) High-resolution Co $2 p$ scan shows characteristic strongly suppressed shake-up satellite peaks at $+5.5 \mathrm{eV}$ and $+9 \mathrm{eV}$, as compared to $\mathrm{CoO}$. The $\mathrm{O} 1 \mathrm{~s}$ peak shows a slight shoulder, which can be attributed to surface oxygen. (c) To determine the valence band offset, the spectra for pure $\mathrm{MgAl}_{2} \mathrm{O}_{4}$ (red shading) and $\mathrm{Co}_{3} \mathrm{O}_{4}$ (blue shading) were scaled, offset, and added together to create a simulated fit (purple line) to the measured valence band spectra for a heterostructure of $13 \AA \mathrm{Co}_{3} \mathrm{O}_{4}$ on $\mathrm{MgAl}_{2} \mathrm{O}_{4}$ (black open circles). The residual difference is also plotted (black line)....31

Figure 3.7: Valence band structure (a) Measured XPS valence band for a 250- $\AA$-thick sample is shown by open circles, with the envelope of the 5-peak fit shaded blue. Indicated by the solid black line, calculated density of states (DOS) for bulk $\mathrm{Co}_{3} \mathrm{O}_{4}$ with $\mathrm{U}\left(\mathrm{Co}^{2+}\right)=4 \mathrm{eV}$ and $\mathrm{U}\left(\mathrm{Co}^{3+}\right)=0 \mathrm{eV}$ agrees well with experimental features. (b) Site- and orbital-projected DOS for $\mathrm{U}\left(\mathrm{Co}^{2+}\right)=4 \mathrm{eV}$ and $\mathrm{U}\left(\mathrm{Co}^{3+}\right)=0 \mathrm{eV}$ 33 xvi 
Figure 3.8: Pseudodielectric function $\langle\varepsilon\rangle$ of a $220-\AA$-thick $\mathrm{Co}_{3} \mathrm{O}_{4}$ film on spinel. Symbols: Experimental data. Lines: Fit with the dielectric function shown in Figure 3.9 35

Figure 3.9: Real (dashed) and imaginary (solid) parts of the dielectric function $\varepsilon$ of $\mathrm{Co}_{3} \mathrm{O}_{4}(220-\AA \AA$-thick film grown by MBE) from the near-IR to the nearUV, showing peaks due to interband electronic transitions. The band gap

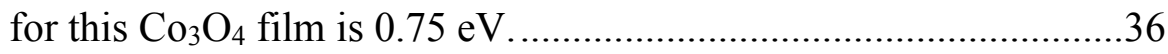

Figure 3.10: Ellipsometric angles for a 220- $\AA$-thick $\mathrm{Co}_{3} \mathrm{O}_{4}$ film on spinel in the midinfrared spectral region at five angles of incidence from $60^{\circ}$ to $80^{\circ}$. (Symbols: Experimental data. Lines: Fit with a Lorentz oscillator expansion of the $\mathrm{Co}_{3} \mathrm{O}_{4}$ lattice absorption.)

Figure 3.11: Real (dashed) and imaginary (solid) parts of the dielectric function of $\mathrm{Co}_{3} \mathrm{O}_{4}$ in the mid-IR, showing lattice absorption by three infrared-active phonons. 38

Figure 3.12: SQUID magnetometer: Magnetization of a 240- $\AA$-thick $\mathrm{Co}_{3} \mathrm{O}_{4}$ (110) film was measured as a function of temperature under zero-field-cooled conditions (blue circles) with constant in-plane magnetic field of $1 \mathrm{~T}$. Field-cooled data show similar characteristics. The onset of antiferromagnetism is labeled at $49 \mathrm{~K}$. Inset image shows G-type antiferromagnetic ordering in the spinel structure. The overall negative magnetization is from the diamagnetic contribution from the substrate. 
Figure 4.1: (a) In situ RHEED of the crystalline STO and alumina surfaces. (b) X-ray reflectivity of structure and interface with inset epitaxial relationship of $\gamma$-alumina on STO (001). (c) Bragg-Brentano scans along the (001) direction.

Figure 4.2: High-resolution electron micrograph in cross-section geometry confirming abrupt interface between highly crystalline epitaxial alumina on STO substrate. 46

Figure 4.3: Core level (a) and valence band (b) spectroscopy for the following: STO substrate (red), $3 \mathrm{~nm}$ alumina/STO (purple), and $7 \mathrm{~nm}$ alumina/STO (blue) 47

Figure 4.4: (a) Peak decomposition of the Ti 2p core level in STO allows comparison of fully oxidized and reduced Ti. (b) Analysis of reduced titanium based on XPS Ti $2 p$ spectra for different samples of different thickness and growth temperature. 48

Figure 4.5: (a) Sheet resistance of MBE-grown alumina/STO samples grown with deposition temperatures of $700^{\circ} \mathrm{C}\left(6 \mathrm{~nm} \mathrm{Al}_{2} \mathrm{O}_{3}\right)$ and $400^{\circ} \mathrm{C}(4 \mathrm{~nm}$ $\left.\mathrm{Al}_{2} \mathrm{O}_{3}\right)$. Annealing the $700^{\circ} \mathrm{C}\left(6 \mathrm{~nm} \mathrm{Al}_{2} \mathrm{O}_{3}\right)$ sample in 1 Torr of $\mathrm{O}_{2}$ at $400^{\circ} \mathrm{C}$ for 5 minutes turned the sample's $R(T)$ into an insulating behavior below $200 \mathrm{~K}$. (b). Temperature dependent Hall carrier density of $700^{\circ} \mathrm{C}$ $(6 \mathrm{~nm})$ sample before and after the annealing in $\mathrm{O}_{2}\left(1\right.$ Torr, $400^{\circ} \mathrm{C}$ for 5 minutes). Hall coefficient was measured using a $\pm 5 \mathrm{~T}$ magnetic field.49 
Figure 5.1: Epitaxy of EuO on $\mathrm{SrTiO}_{3}$ (001). (a) Atomic model of the rocksalt / perovskite heterointerface. (b) Reciprocal space map of the STO (002) and $\mathrm{EuO}(113)$ peaks for $7 \mathrm{~nm} \mathrm{EuO}$ on STO. The EuO rocksalt unit cell is rotated $45^{\circ}$ with respect to the surface unit cell of the perovskite. (c) High-angle annular-dark-field scanning transmission electron microscopy [100]-projection image of the EuO/STO interface. (d) Corresponding false color map shows a distribution map from the Ti Ledge fit $\left(\mathrm{SrTiO}_{3}\right.$, red; $\mathrm{SrTiO}_{3-\delta}$, green). (e) Ti-L coefficient as a function of position shows a sharp peak at the interface. (f) Overall schematic, including the bulk STO substrate (red), layer of STO with oxygen vacancies (green), EuO film (blue), and capping layer (gray). Not to scale.

Figure 5.2: (a) Magnetoresistance (MR) data measured in a perpendicular magnetic field at $20 \mathrm{~K}, 100 \mathrm{~K}$, and $300 \mathrm{~K}$. Solid lines indicate fits to the data. The MR increases linearly with the magnetic field at $20 \mathrm{~K}$, quadratically at $100 \mathrm{~K}$, and is field-independent at room temperature. (b) Linear $c_{1}$ and quadratic $c_{2}$ MR fit coefficients for the same film as a function of temperature. (c) Field-cooled magnetization $M$ of a similar 7-nm EuO film as a function of temperature at constant in-plane magnetic field of 0.01 T. Inset: corresponding magnetization loop measured at 10 K.59 
Figure 5.3: Resonant soft-X-ray ARPES of 2-nm EuO/STO heterointerface through the Ti $L$-edge. (a) XAS spectrum. (b) Resonant photoemission from the valence band as a function of excitation energy. Intensity the near- $E_{\mathrm{F}}$ region is scaled up by $\sim 30$. (c) Resonant intensity for constant $E_{\mathrm{B}}$ in the valence band, Eu level and 2DEG. The valence band and 2DEG signals follow the Ti $L$-edge XAS spectrum that confirms their Ti-derived character. (d,e) SX-ARPES images at $h v=460.3$ and $466 \mathrm{eV}$ enhancing the $d_{\mathrm{xy}}$ and $d_{\mathrm{yz}}$-derived states, respectively. The intensity waterfalls are reveal polaronic nature of the interface charge carriers. (f) Fermi surface of the interface states measured at $h v=466 \mathrm{eV}$. .64

Figure 6.1: Graphical abstract from Ref. [185]. 67

Figure 6.2: RHEED patterns for (a) Sr Zintl template on Si along Si \{110\}, (b) $6 \mathrm{~nm}$ STO along STO $\{110\}$, and (c) $90 \mathrm{~nm}$ BTO along $\{110\}$. (d) The BTO $<210>$ surface unit cell was monitored during growth. An extra streak appears in the RHEED pattern for the Ti-rich surface, indicating a doubling of the surface unit cell size. (e) In a line scan taken during one unit cell of BTO deposition (120 s), the extra streak disappears for stoichiometric BTO. 69

Figure 6.3: Atomic force microscopy (AFM) of the as-grown sample (a) reveals many small particles (presumably $\mathrm{BaOx}$ ) on the surface. After soaking in water for 4 hours (b), few particles remain. 69 
Figure 6.4: (a) Electro-optical characterization quantifies rotation $(\delta)$ of the polarization $\left(\theta_{\mathrm{P}}\right)$ of a laser beam transmitted through pairs of lithographically defined electrodes. (b) Thickness-normalized electrooptic response $\delta^{\prime}=\delta /\left(E_{A C} \cdot t\right)$. as a function of offset (DC) field for a-axis, mixed, and c-axis films.

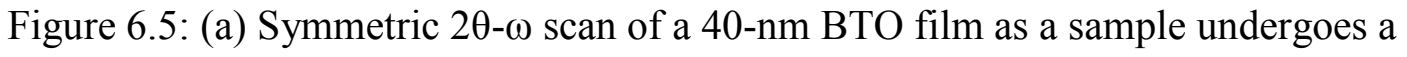
series of $30 \mathrm{~min}$ RTA in $\mathrm{O} 2$ at incremental temperatures $\left(500-850^{\circ} \mathrm{C}\right)$. (b) Corresponding rocking curves about the (002) BTO Bragg peak. (c) Corresponding FWHM and out-of-plane lattice constants over the annealing series

Figure 6.6: (a) Leakage current and (b) symmetric 2 $\theta-\omega$ scan for a series of films with varied growth conditions 72

Figure 7.1: (a) In-plane electrode pairs were patterned with various angles $\theta_{\mathrm{E}}$ defined relative to the BTO $[100] /[010] /[001]$ direction. As described in the text, the sinusoidal measuring and static poling voltages $V_{\mathrm{pp}}+V_{\mathrm{DC}}$ are applied across the electrodes. (b) For electro-optic transmission measurements, a half-wave plate is used to set the incident linear polarization. After passing through the sample, the light becomes elliptically polarized, which is transformed into linearly polarized light after passing a quarterwave plate. Finally, a Glan-laser prism is used to determine the orientation of the transmitted polarization. (c) Corresponding states of the polarization along the path of the laser beam are visualized as the ellipse traced out by the light's electric field vector with (red) and without (blue) an external electric field. After transmission through the sample and quarter-wave plate, the polarization is rotated by angle $\delta .79$ 
Figure 7.2: (a) Medium-angle annular dark-field scanning transmission electron microscopy (MAADF-STEM) images of BTO thin films deposited by MBE, (b) PLD, (c) RF sputtering, and (d) CVD. Pores are labelled "P". The boundaries of representative grains are outlined with solid lines. In (c), the boundary between epitaxial and polycrystalline BTO is indicated with dashed line. 82

Figure 7.3 (a) Schematic of $90^{\circ}$ domain structure, with the BTO tetragonal axes indicated by white arrows. (b) X-ray diffraction patterns for different measurement configurations for epitaxial BTO deposited by MBE and (c) CVD. A sum of 3 Voigt functions has been fitted to each pair of scans (solid lines), with vertical dashed lines indicating the positions of the peaks corresponding to the BTO $a$ and $c$ lattice parameters.......85

Figure 7.4: (a) The field-normalized induced rotation $\delta$ ' was measured (symbols) and simulated (lines) for varied orientation angle $\theta_{\mathrm{E}}$ (defined in Figure 7.1(a)) of in-plane electric field for BTO films deposited by MBE and (b) CVD. Details on error bars can be found in the methods section. 87

Figure 7.5: (a) The field-normalized induced rotation $\delta$ ' measured as a function of a DC offset bias for BTO deposited by MBE and (b) CVD. Sigmoidal fits (solid lines) are utilized to extract saturation and remanent response, ${ }^{\prime}{ }_{s}$ and $\delta^{\prime}{ }_{r}$ respectively, as well as the coercive field $E_{\mathrm{c}}$. Sweep direction is indicated by arrows, with sigmoidal fits to the data described in Appendix B .89 
Figure 7.6: (a) Effective Pockels coefficient $r_{\text {eff }}$ vs porosity, (b) anisotropy ratio of Pockels tensor elements $r_{42} / r_{33}$ vs tetragonal distortion $\gamma$, where cubic BTO corresponds to $\gamma=0$, and (c) remanence ratio or squareness factor $\delta^{\prime}{ }_{r} / \delta^{\prime}{ }_{s}$ vs. grain size $e d r$. Open symbols: polycrystalline, filled symbols: textured/epitaxial, half-filled indicates a mixture. 90

Figure 8.1: Plot of heat of formation of most stable oxide vs. work function for common metallic elements used in this dissertation and in Ref. [184]. The elements naturally group into three categories as subdivided by the solid lines creating three occupied quadrants in the plot. The solid boundaries are based on several empirical reports of oxidation of metals on $\mathrm{SrTiO}_{3}$ and very roughly corresponds to the formation energy of an oxygen vacancy in $\mathrm{SrTiO}_{3}$ and the electron affinity (work function) of ntype $\mathrm{TiO}_{2}$-terminated $\mathrm{SrTiO}_{3}$ 97

Figure 8.2:Pockels coefficients for oxide thin films. .98

Figure 8.3: (a) Schematic from Ref. [252] and (b) experimental realization of reservoir computing using Mach-Zehnder interferometer on the kilometer scale. [253].......................................................... 100

Figure A.1: XPS Eu (a) $4 d$ and (b) $3 d$ core level measurements for varied substrate temperature and oxygen partial pressure. 103

Figure A.2: (a) X-ray diffraction coupled scans with corresponding (b) in-plane reciprocal space map and positions in reciprocal space for (c) STO and (b) $\mathrm{EuO}$ with a $45^{\circ}$ rotation of the surface unit cell. 104

Figure A.3: MLLS fitting. Red is $\mathrm{EuO}$ and Green is $\mathrm{Eu}_{2} \mathrm{O}_{3}$. Only a little $\mathrm{Eu}^{3+}$ is present in the disordered region. (Reference spectra taken from Mairoser et al. [194]). 105 Xxiii 
Figure A.4: (a) Sheet resistance for a 7-nm EuO film as a function of temperature. (b) Hall resistance $R_{\mathrm{XY}}$ for a $7-\mathrm{nm}$ EuO film at $120 \mathrm{~K}$. Solid lines indicate linear fits. 106

Figure A.5: High-angle annular dark-field scanning transmission electron microscopy [110]-projection image of the EuO/STO interface. 106

Figure A.6: (a) To determine the valence band offset, the spectra for pure $\mathrm{EuO}$ (red shading) and STO (blue shading) were scaled, offset, and added together to create a simulated fit (purple line) to the measured valence band spectra for a heterostructure of $1 \mathrm{~nm} \mathrm{EuO} \mathrm{on} \mathrm{STO} \mathrm{(black} \mathrm{open} \mathrm{circles).}$

(b) Schematic of band alignment. 107

Figure A.7: The partial density of states projected onto Ti ions at each layer. Interface $\mathrm{TiO}_{2}$ layer is at top and middle bulk $\mathrm{TiO}_{2}$ layer is at bottom. $\mathrm{d}_{\mathrm{xy}}$ orbitals are marked as black line. $\mathrm{d}_{\mathrm{xz}}$ and $\mathrm{d}_{\mathrm{yz}}$ orbitals are marked as red line. $\mathrm{d} 3 \mathrm{z} 2-\mathrm{r} 2$ orbitals are marked as blue and dx2-y2 orbitals are marked as magenta. 108

Figure A.8: Resonance photoemission spectra of valence band near Eu 3d threshold. Strong resonance of the $\mathrm{Eu}^{2+}$ states in the valence band at $\mathrm{h} v=1128 \mathrm{eV}$ and $\mathrm{Eu}^{3+}$ states at $\mathrm{hv}=1130 \mathrm{eV}$. No resonating states at the $E_{\mathrm{F}}$. (b) Valence band spectrum at $\mathrm{h} v=457 \mathrm{eV}$ shows the prevalence of $\mathrm{Eu}^{2+} .109$

Figure A.9: Photoelectron images of 2DES for hv $=460.3$ and $466 \mathrm{eV}$ for a burnt spot of the sample surface. 110

Figure A.10: Photoelectron images of 2DES for $\mathrm{h} v=460.3$ and $466 \mathrm{eV}$ for a fresh spot of the sample surface. 111

Figure A.11: "0" state. 112 
Figure B.1: Bright-field scanning transmission electron microscopy (STEM) images of barium titanate (BTO) on strontium titanate $(\mathrm{STO}) / \mathrm{Si}$ pseudosubstrates deposited by (a) molecular beam epitaxy (MBE), (b) pulsed laser deposition (PLD), (c) RF sputtering, and (d) chemical vapour deposition (CVD). Brighter areas in the film correspond to reduced film density, pores, labeled "p".

Figure B.2: (a) Atomic force microscopy images for BTO deposited by MBE, (b) PLD (c) RF sputtering, (d) RF sputtering (no buffer), and (e) CVD. Root mean square (RMS) surface roughness values are summarized in Table B. 1 113

Figure B.3: (a) X-ray diffraction patterns for BTO deposited by MBE, PLD, RF sputtering on STO/Si pseudosubstrate (green up triangles), RF sputtering directly on Si (orange down triangles), and CVD. Out-of-plane and grazing incidence in-plane scans are shown by filled and open symbols, respectively. A sum of three Voigt functions has been fitted to each pair of scans, as shown by solid lines. (b) Corresponding rocking curves of the BTO (002) reflection (not available for BTO sputtered directly on $\mathrm{Si})$. The rocking curves full width at half maximum (RC FWHM) are summarized in Table B.1. 114 
Figure B.4: (a) The field-normalized induced rotation $\delta$ ' was measured (symbols) and simulated (lines) for varied orientation $\theta_{\mathrm{E}}$ of in-plane electric field for BTO films deposited by MBE, PLD, RF sputtering on STO/Si pseudosubstrate (green up triangles), RF sputtering directly on $\mathrm{Si}$ (orange down triangles), and CVD. (b) The orientation of the incident polarization of the linearly polarized light at which the maximum in $\delta$ ' is observed, $\theta_{\mathrm{p}, \max }$, was also measured and simulated. (c) Example of data fitting to select best-fit parameters $r_{42}$ and $r_{33}$. Squares: simulated points, contours: 2-d Gaussian fit. 115

Figure B.5: (a) The field-normalized induced rotation $\delta$ ' measured as a function of a DC offset bias for BTO deposited by MBE, PLD, RF sputtering on STO/Si pseudosubstrate (green up triangles), RF sputtering directly on Si (orange down triangles), and CVD. Sigmoidal fits (solid lines) are utilized to extract saturation and remanent response, $\delta_{\mathrm{S}}$ 'and $\delta_{\mathrm{R}}$ ' respectively, as well as the coercive field $E_{\mathrm{c}}$ 
Figure B.6: (a) Schematic of the geometry used for simulating the electric field distribution in porous BTO layers. While a cylindrical pore is shown here, we also considered rectangular cuboid pores. (b) Example of the electric field distribution in a cross-sectional plane as indicated in Fig. (a). The simulations were carried out using the commercial finiteelement solver COMSOL Multiphysics. The color scale indicates the electric potential $\varphi$, with arrows indicating magnitude and direction of the corresponding electric field. Simulation parameters and results are summarized in Table B.3(c) Correction factors $c_{\mathrm{P}}$ are calculated for varied porosities $p$, dielectric constants $\varepsilon_{\mathrm{BTO}}$, and pore cross-section shapes (circle, square, or rectangular slab of width $w$ (inset))........116 


\section{Chapter 1: Introduction}

\subsection{NEW OPPORTUNITIES WITH OXIDE INTERFACES}

After more than 50 years, the steady scaling of semiconductor device dimensions following "Moore's Law" [1] is swiftly approaching the limits of conventional materials. Rather than simply following the miniaturization approach, new materials have been proposed to replace $\mathrm{Si}$ channels, $\mathrm{SiO}_{2}$ gate dielectrics, and metal interconnects with high mobility channels, high- $\kappa$ dielectrics, and optical interconnects, respectively. [2]

One candidate class of materials is heterostructures based on oxides of transition metals with perovskite crystal structure [3-8]. Owing to the exquisite, atomic level control of layer design, afforded by new developments in oxide epitaxy, these systems are expected to enable militarily significant and commercially valuable products. The unprecedented richness of physical phenomena observed in these materials systems, stems from the delicate balance of multiple interactions that control the quantum behavior of $\boldsymbol{d}$ - and sometimes $\boldsymbol{f}$-electrons in the unfilled shells of the transition metal ion. In bulk materials, this is the origin of magnetism, superconductivity, ferroelectricity and several other related effects [9]. On the other hand, the properties of epitaxial interfaces are controlled by strain, band alignment, and crystal imperfections that may affect the longrange as well as the short-range order. Symmetry lowering at the interface creates entirely new environments for the electrons of the "active" atomic species that are not realizable in the bulk environments. In heterostructures, the role of substrate/overlayer interaction is a major contributor to the performance of oxide materials in the two-dimensional limit.

While the perovskite oxides $\left(A B \mathrm{O}_{3}\right)$ were initially of interest for high- $\mathrm{k}$ gate dielectrics, oxide thin films are of increasing importance in a diverse range of applications, including for example spintronics and photonics. In this dissertation, I 
explore two classes of materials, (1) the high mobility two-dimensional electron gas at epitaxial oxide interfaces, and (2) thin epitaxial electro-optic oxides.

\subsection{TWO-DimenSIONAL ELECTRON GAS AT OXIDE INTERFACES}

Contents of this Section were published in Ref. [10]. *

A recently discovered revolutionary class of polar oxide heterostructures [11] holds tremendous promise for exploiting the physical properties of the novel quasi twodimensional electron gas (2DEG) formed at the oxide/oxide interface. [10] The fundamental scientific understanding of these oxide heterostructures is of significant fundamental importance. After this pioneering work, the origin of the 2DEG has been widely investigated and can be attributed to at least three interfacial phenomena, as illustrated in Figure 1.1: (i) electronic reconstruction, as suggested for the original $\mathrm{LaAlO}_{3} / \mathrm{SrTiO}_{3}(\mathrm{LAO} / \mathrm{STO})$ structure [11]; (ii) electrically active defects [12-14]; or (iii) stoichiometry deviations [14,15].

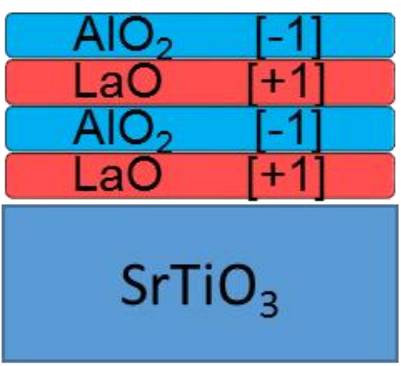

(a)

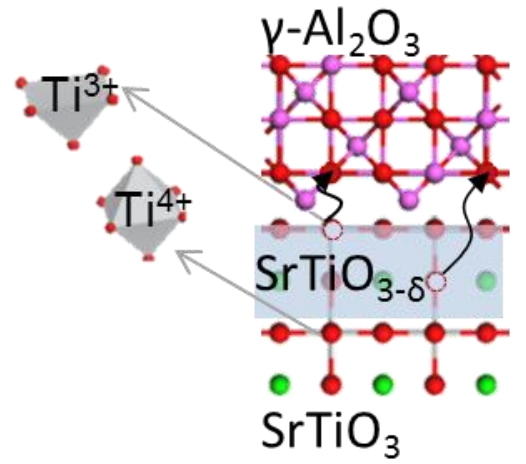

(b)

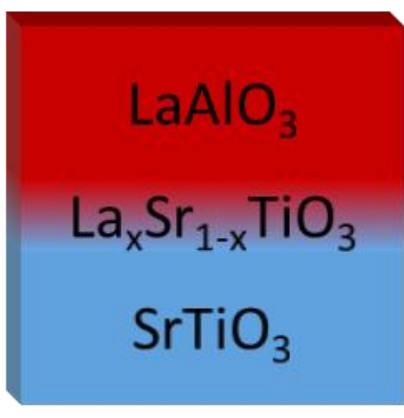

(c)

Figure 1.1: Highly conductive interfaces can be formed between two insulating oxides due to (a) electronic reconstruction, (b) oxygen vacancies, and (c) stoichiometry deviations.

${ }^{*}$ Contributions: AAD, KJK, and KDF contributed equally to this publication. 
Although the 2DEG is usually thought of as localized at the interface, the spatial extent of the gas has been found to vary from a depth of a few nanometers to hundreds of micrometers. [16] The varying spread of the $2 \mathrm{DEG}$ is due to many factors, including oxygen vacancy concentration, [16] temperature of the system, [12] charge density of the gas, [17] amount of cationic exchange, [18] and ionic relaxation at the interface. [19-21]

Experiment and theory show that the $2 \mathrm{DEG}$ at the oxide/oxide interface has many exotic features. It can be paramagnetic, ferromagnetic or even superconducting, [22-25], [97, 99] with strong Rashba splitting leading to a controllable magnetic moment. [26-28]. It has been demonstrated that various modifications of the LAO/STO heterostructure also lead to a variety of interesting effects. Arras et al. used density functional theory (DFT) to calculate the effect of metallic layers on LAO/STO heterostructures. For a thin STO substrate (2.5 unit cells (UC)), 1 monolayer (ML) of Ti deposited on LAO lead to the vanishing of the electric field in LAO; even given this, the LAO/STO interface is still conducting, due to the migration of charge to the lowest unoccupied states that exist at the bottom of the STO conduction band; moreover, the Ti metallic layer and the interface are both magnetic. For thicker STO $(6.5 \mathrm{UC})$, the system is the same, except that the interface is nonmagnetic; the authors ascribe the magnetization of the interface for thinner STO to quantum confinement effects. Upon the addition of more MLs of Ti metal on the LAO surface, the conducting states do not change appreciably, but the magnetization of the Ti metal is reduced; by $3 \mathrm{ML}$ of $\mathrm{Ti}$, the surface metal (and thus the entire system) is nonmagnetic. The authors also tried different metallic contacts (single ML of $\mathrm{Na}, \mathrm{Al}, \mathrm{Fe}, \mathrm{Co}, \mathrm{Cu}, \mathrm{Ag}, \mathrm{Pt}$ and $\mathrm{Au}$ ). The field in LAO was not always reduced to zero; in fact, for the Au contact, the electric field was enhanced! This is due to the large work function of $\mathrm{Au}$, which places the Fermi energy mid-gap in STO; therefore, no charge is transferred to the STO/LAO surface and the field in LAO is not destroyed [29]. 
Although most of the experiments were done on LAO grown on bulk STO, there have also been reports of the 2DEG found in LAO/STO heterostructures deposited on $\mathrm{Si}$, paving the way for semiconducting devices utilizing the properties of the oxide 2DEG. [30] Levy and co-authors used atomic force microscope lithography to induce a reversible metal/insulator transition of the interface. [31,32]

In this section, we identify oxide systems which exhibit interfacial conductivity (often two dimensional) stemming from (one or more of) three separate origins: electronic reconstruction, cation exchange, and oxygen vacancies.
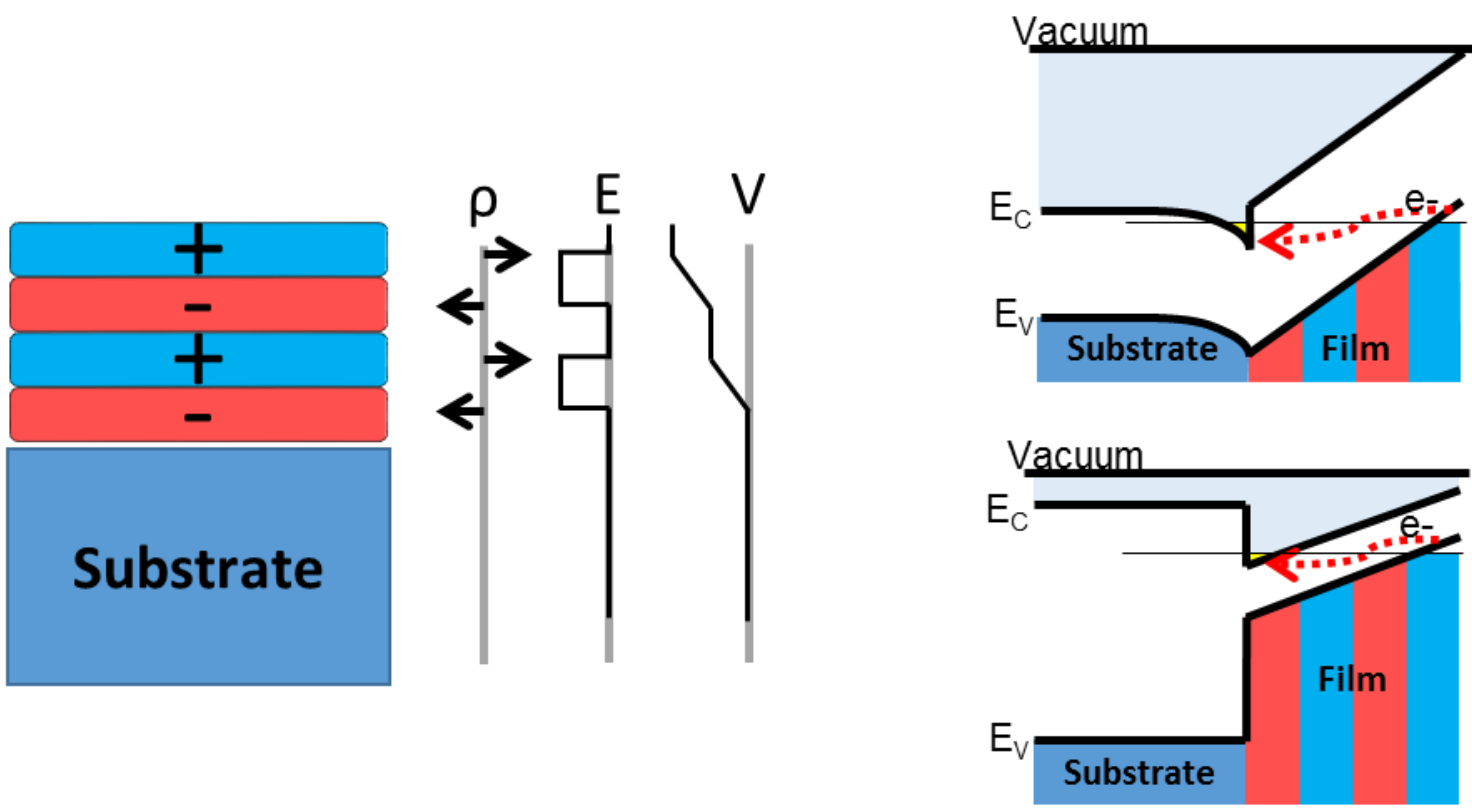

Figure 1.2: (a) Illustration of electrostatic model for the polar catastrophe. (b) and (c) illustrate critical thickness for electronic reconstruction.

Discussions of the origins of the oxide 2DEG often start with polar catastrophe and electronic reconstruction. [33-35] Left uncompensated, a large electric field is builtup in a polar oxide such as LAO; the alternating positively charged $\mathrm{LaO}$ and negatively charged $\mathrm{AlO}_{2}$ layers lead to a ramping up of the electrostatic potential that grows without 
limit (Figure 1.2). Due to the large energy cost of this internal field, and to avoid dielectric breakdown, the heterostructure must find a way to compensate this diverging electrostatic potential. One way to avoid the polar catastrophe is electronic reconstruction, where the electronic charge migrates to the interface to eliminate the field once a critical thickness is reached (Figure 1.2).

\subsubsection{Oxygen Vacancies in LAO/STO}

Surprisingly, conductivity has been demonstrated at the interfaces between the STO surface and oxide thin films of the polar, nonpolar, epitaxial and amorphous varieties. [36-38] These studies have highlighted a need to investigate the role of defects in the formation of the oxide $2 \mathrm{DEG}$ and its corresponding transport properties. Interfacial redox reaction of STO can n-dope the material to stabilize a confined conducting layer. Thus, interfacial defect formation has been studied alongside electronic reconstruction the LAO/STO system.

An alternative explanation for the conductivity at the n-type interface is due to surface $\mathrm{O}$ vacancy formation. These $\mathrm{O}$ defects introduce electrons to the system, which settle at the STO conduction band bottom and kill the polar field in LAO, creating the interfacial 2DEG. The enthalpy of formation for these vacancies decreases with increasing LAO thickness, spontaneously forming at the LAO critical thickness, which shows why the system is not insulating under the critical thickness but becomes insulating suddenly at the critical thickness. In contrast, at the p-type interface, the enthalpy of formation of surface $\mathrm{O}$ vacancies never becomes negative, so the 2DEG does

not form at any thickness [39]. O vacancies at the p-type interface create a defect state just above the Fermi energy, which also causes the interface to become insulating [22]. 
Slabs of pure LAO that show electronic reconstruction can also be made insulating by the addition of $\mathrm{O}$ vacancies at the surface that donate electrons to the system [40].

Ferrari et al. examined the less-traditionally studied $\mathrm{AlO}_{2} / \mathrm{TiO}_{2}$ interface for a $\mathrm{LAO} / \mathrm{STO}$ heterostructure, which has been found to be more stable when $\mathrm{O}$ vacancies are included [41]. The heterostructure consisted of $10 \mathrm{UC}$ of both LAO and STO, with 10 ML of vacuum to prevent slab-slab interactions; the lateral size of the cell was $(\sqrt{2} x \sqrt{2})$ to include more $\mathrm{O}$ to perform $\mathrm{O}$ vacancy studies. For $50 \%$ and $25 \% \mathrm{O}$ vacancies at the $\mathrm{AlO}_{2}$ $/ \mathrm{TiO}_{2}$ interface, the $\mathrm{AlO}_{2} / \mathrm{TiO}_{2}$ interface contained a $2 \mathrm{DEG}$ that was found occupied the interfacial Ti $d$ states, and the $\mathrm{O}$ at the LAO surface contained 2D hole states. [42]

Kalabukhov et al. experimentally investigated optical, electrical, and microstructural properties of heterointerfaces between two thin-film insulating perovskite materials, $\mathrm{SrTiO}_{3}$ (STO) and $\mathrm{LaAlO}_{3}$ (LAO), deposited at different oxygen pressure conditions. Cathode and photoluminescence experiments suggested that oxygen vacancies were formed in the bulk STO substrate during the growth of LAO films, resulting in high electrical conductivity and mobility values. In both high and low oxygen pressure interfaces, the electrical Hall mobilities followed a similar power law dependence as observed in oxygen reduced STO bulk samples. The results were confirmed on a microscopic level by local strain fields at the interface reaching $10 \mathrm{~nm}$ into the STO substrate [13]. Electrical measurements were made in a four-point van der Pauw configuration in the temperature range $2-300 \mathrm{~K}$ and in magnetic field up to $5 \mathrm{~T}$. Gold contact pads were fabricated by sputtering using a $\mathrm{Ti}$ adhesion layer. The temperature dependence of the sheet resistance $R X X$, the Hall mobility $\mu_{H}$, and the charge-carrier density $n_{S}$ are presented in Ref. [13]. The results strongly suggest that oxygen vacancies in STO are responsible for the conductivity in LAO/STO heterostructures prepared even at high oxygen pressures. 
Similarly, based on transport, spectroscopic, and oxygen-annealing experiments, Siemons et al. concluded that extrinsic defects in the form of oxygen vacancies introduced by the pulsed laser deposition (PLD) process were the source of the large carrier densities [12]. Annealing experiments showed a limiting carrier density. In addition, a model was introduced that explains the high mobility based on carrier redistribution due to an increased dielectric constant. Measurement of the electronic properties of the interfaces created by depositing $\mathrm{LaAlO}_{3}$ on $\mathrm{SrTiO}_{3}$ showed electronic properties similar to those found originally by Ohtomo and Hwang [11]. Also, UPS spectra showed states at the Fermi level, indicating a conducting interface. The number of these states was reduced when the sample was oxidized, suggesting that oxygen vacancies played an essential role in supplying the charge carriers. This was further confirmed by NEXAS and VUV-SE measurements, which showed more $\mathrm{Ti}^{3+}$ for samples made at lower pressures. It appeared that the vacancies were created by the pulsed laser deposition (PLD) process itself where relatively high energy particles sputter off oxygen. [43] Annealing samples in atomic oxygen reduced the number of carriers but kept the mobility the same. The dependence of sheet carrier density as a function of temperature was changed dramatically. To determine the electron location, the authors calculated the potential and the carrier density in the $\mathrm{SrTiO}_{3}$ as a function of distance from the interface and concluded that electrons moved into the pristine $\mathrm{SrTiO}_{3}$ over large distances, mainly due to the high dielectric constant of $\mathrm{SrTiO}_{3}$ at low temperatures.

Chen et al. deposited several insulating amorphous oxide thin films by PLD. [44] While amorphous LAO, STO, yttria-stabilized zirconia (YSZ) films deposited on STO rendered metallic interface, $\mathrm{La}_{7 / 8} \mathrm{Sr}_{1 / 8} \mathrm{MnO}_{3}$ interfaces remained highly insulating. Along with the thickness-dependence of conductivity, this suggests that sputtering due to the high energy of incident ions cannot account solely for the formation of oxygen vacancies. 
Therefore, interfacial chemical reactions play an imporatnt role in the formation of the STO oxygen vacancy 2DEG, with possible enhancement due to the sputtering effect. This is consistent with studies of amorphous LAO, alumina, and $\mathrm{YAlO}_{3}$ deposited by ALD. [45]

In conclusion, interfacial oxygen vacancies in STO contribute to the formation of a conducting layer. The confinement of this layer depends on the chemical reactivity of the film. The demonstration of room-temperature 2DEG formation based on oxygen vacancies is particularly promising for future applications. Thin conductive layers have been demonstrated at the interfaces of several oxide systems. In many cased the twodimensional nature of the carriers (two-dimensional electron gas or 2DEG) has been clearly demonstrated in transport measurements. For the 2DEG at the oxide/oxide interface, several factors, including electronic reconstruction, oxygen vacancy concentration, and level of cationic exchange, have been shown to influence the localization of charge at the interface to varying degrees. The phenomenon of electronic reconstruction has been investigated in LAO/STO heterostructures, as well as other polar and ferroelectric oxides. Study of amorphous and nonpolar oxides on STO has highlighted the role of oxygen vacancies. Cation intermixing can also lead to the formation of thin conductive layers. Rapid advances in experiment and theory alike, promise to provide new insights and discoveries in this fast-growing field.

\subsection{FILMS OF INTEgRATED NONLINEAR OXIDES}

Among the many functionalities found in complex oxides, ferroelectricity is one of the most intensely studied. The first demonstration of ferroelectric switching in synthetic perovskite $\mathrm{BaTiO}_{3}$ [46] was reported in 1946, just 25 years after the first measurement of the electrical hysteresis in the Rochelle salt in 1921. [47] This initial 
bulk characterization phase of research was motivated by the high dielectric constant of $\mathrm{BaTiO}_{3}$. [48] In the ferroelectric phase, rumpling of Ti causes $\mathrm{BaTiO}_{3}$ to lose its center of inversion, as shown in Figure 1.3.
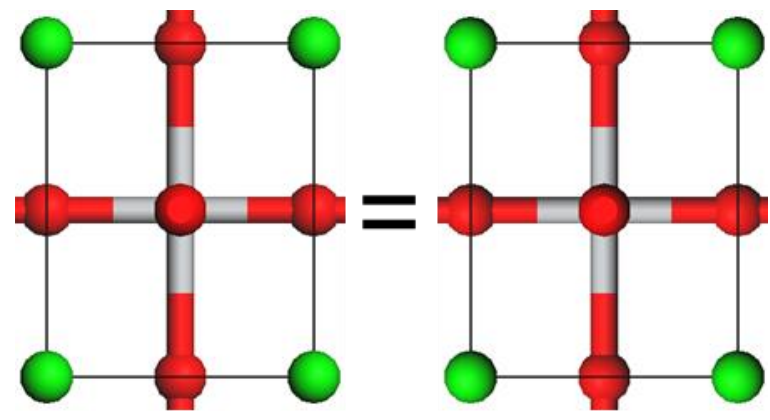

Figure 1.3: Tetragonal $\mathrm{BaTiO}_{3}$ has a center of inversion (top), while ferroelectric $\mathrm{BaTiO}_{3}$ is noncentrosymmetric (bottom).
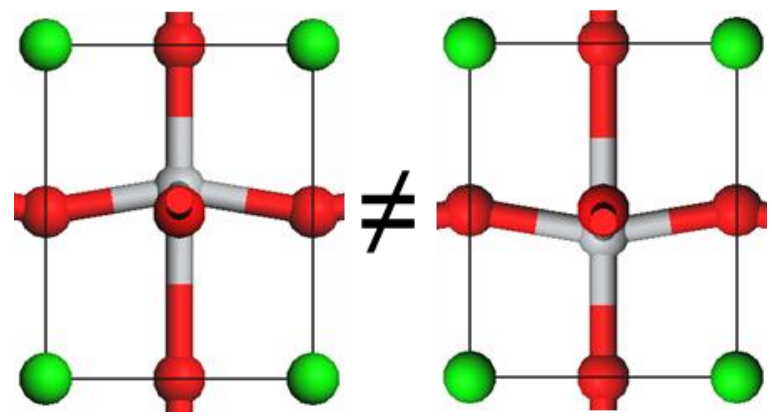
linear electro-optic effect was first demonstrated in 1893 by Friedrich Pockels. [49] Since the 1970s, another ferroelectric perovskite oxide, $\mathrm{LiNbO}_{3}$, has emerged as the material of choice for replacing traditional metal wires with electro-optically modulated optical fiber links. [50,51] As early as the 1980s, [52] a similar need was identified to thwart delays due to scaling limitations of wiring. [53] The solution proposed was to swap metal interconnects with waveguides, nanoscale optical interconnects. [52,54-59] The following development of Si photonics in the 2000s was a key move towards this goal.

This effort to replace metal interconnects with waveguides has been motivated by the prospect of leveraging the speed of optical communication in conjunction with semiconductor logic. Among several approaches, the linear electro-optic (or Pockels) effect offers an attractive route for switching in Si photonics. [60-72] The Pockels coefficients of several candidate materials are shown in Figure 1.4. While silicon can be 
used as a waveguide, this material has a nonzero Pockels coefficient only if strained due to its centrosymmetric structure in bulk. This demonstrates the need for selection of a CMOS-compatible electro-optically active material for development of a hybrid $\mathrm{Si}$ nanophotonics platform. When selecting a candidate material, two main criteria emerge: the material should not only have a large Pockels coefficient for efficient low-power performance, but should also be compatible with Si.

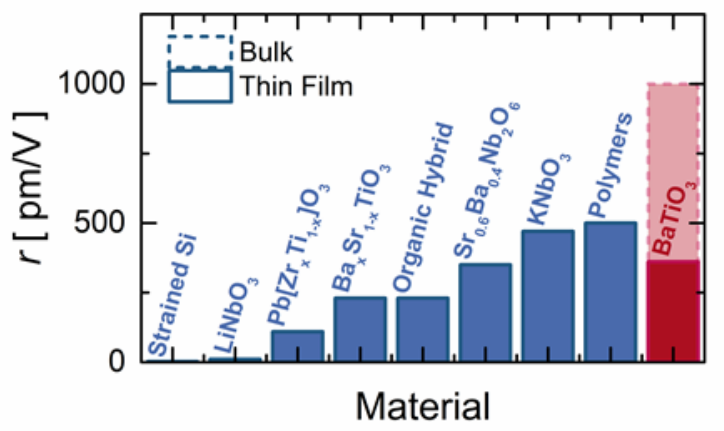

Figure 1.4: Electro-optical Pockels coefficients $r$ for various materials [7375].

The first waveguides based on optical-quality perovskite films were fabricated in 1994 [76] with resulting thinfilm channel electro-optic modulators following by 1997. [77] The substrate of choice was $\mathrm{MgO}$ (001), due to its low refractive index and transparency. [74] A review of ferroelectric films can be found in Ref. [74]. Within a decade, electro-optic modulators based on $\mathrm{BaTiO}_{3} / \mathrm{MgO}$ performance metrics reached electro-optic coefficients of $360 \mathrm{pm} / \mathrm{V}$ and half-wave voltage-length products as small as $0.5 \mathrm{Vcm}$ at telecom wavelength of $1561 \mathrm{~nm}$. [78] The challenge of oxide integration on semiconductors was first addressed in 1998 [79]. In the 2010s, major advances in epitaxial $\mathrm{BaTiO}_{3} / \mathrm{Si}$ were reported, including ferroelectric switching, [80] electro-optically active integrated films, [67] active electro-optic devices, [63,72] and low-loss waveguides. [81] This demonstrates the viability of $\mathrm{BaTiO}_{3} / \mathrm{Si}$ for development of a hybrid Si nanophotonic platform. 


\subsection{OBJECTIVE AND OVERVIEW OF CHAPTERS}

As indicated in the above introduction, emergent phenomena in transition metal oxide films are receiving considerable attention due to the development of techniques for the preparation of well-controlled oxide surfaces. This dissertation focuses on epitaxial oxides of the perovskite, spinel, and rocksalt structure deposited by molecular beam epitaxy. Chapter 2 provides an overview of the main equipment used for film deposition and characterization in the Materials Physics Lab at the University of Texas at Austin and at IBM ZRL.

Because polar oxides are of prominent interest as a mechanism for the formation of the two-dimensional electron gas, Chapter 3 provides details on the growth and characterization of polar semiconductor $\mathrm{Co}_{3} \mathrm{O}_{4}$. A closer look at the role of defects is presented in Chapter 4, where a highly conducting layer is formed at the crystalline $\gamma$ alumina/ $\mathrm{SrTiO}_{3}$ interface due to oxygen vacancies. Building upon these results, Chapter 5 applies the same concept to highly spin-split semiconductor $\mathrm{EuO}$ to explore proximity effects in this functional oxide heterostructure.

Switching to photonics, Chapter 6 examines $\mathrm{BaTiO}_{3}$ films of varied thickness deposited on Si to quantify the Pockels coefficient with respect to crystalline orientation. As a next step, Chapter 7 reports the strong dependence of the Pockels effect in $\mathrm{BaTiO}_{3}$ thin films on their microstructure, and provide guidelines on how to engineer thin films with strong electro-optic response for the development of novel hybrid silicon photonic platform. 

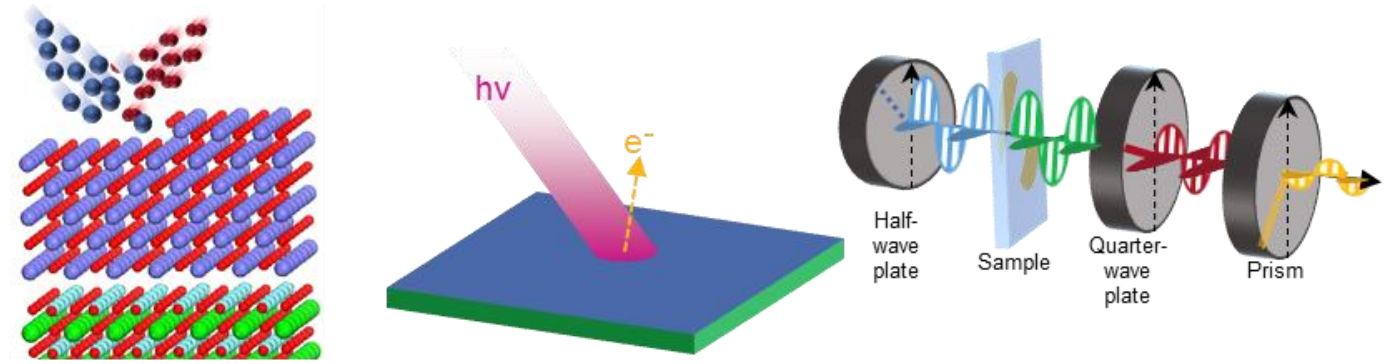

\section{Chapter 3-5: Oxide 2DEG}
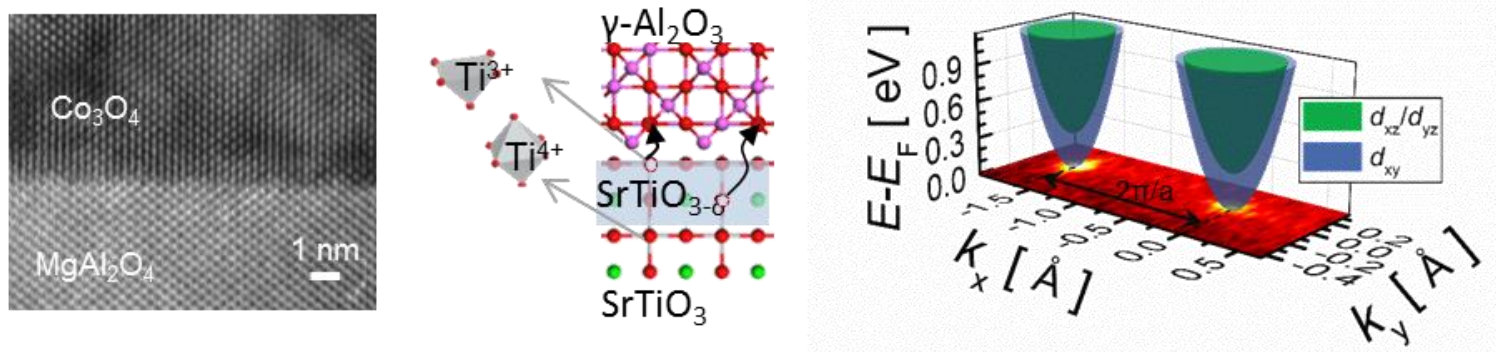

Chapter 6-7: Integrated Nonlinear Oxides
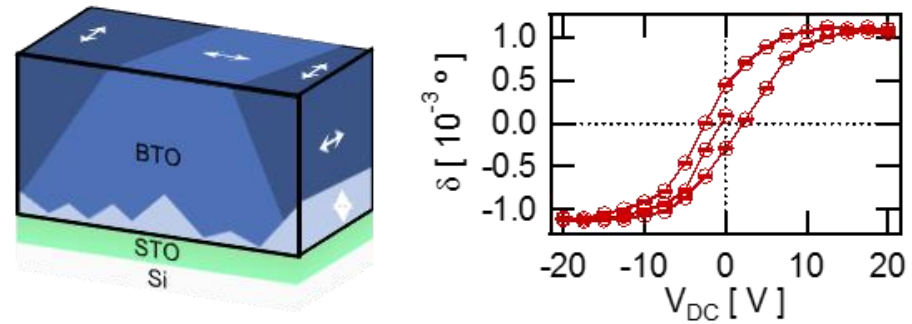

Chaper 8: Summary
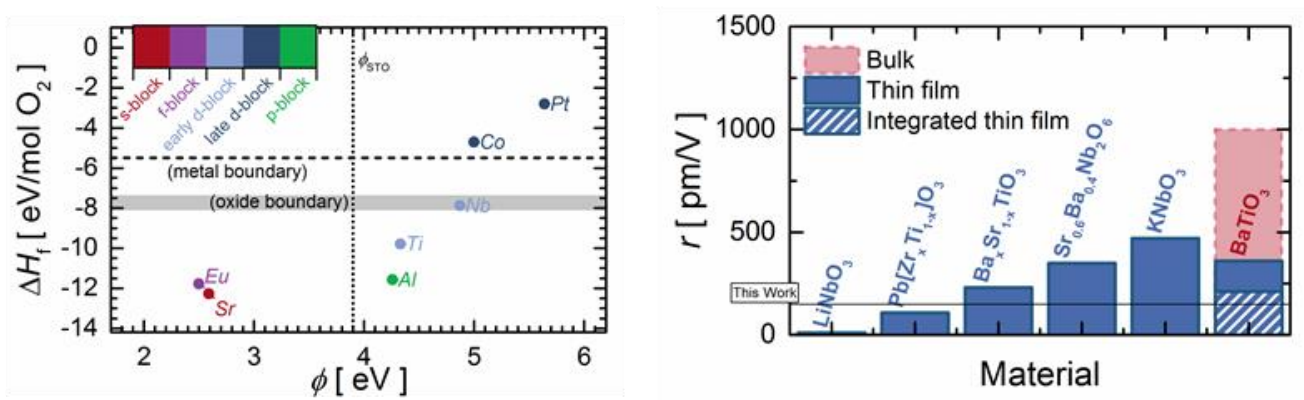

Figure 1.5: Overview of dissertation chapters. 


\section{Chapter 2: Experimental Techniques}

\subsection{Molecular Beam Epitaxy}

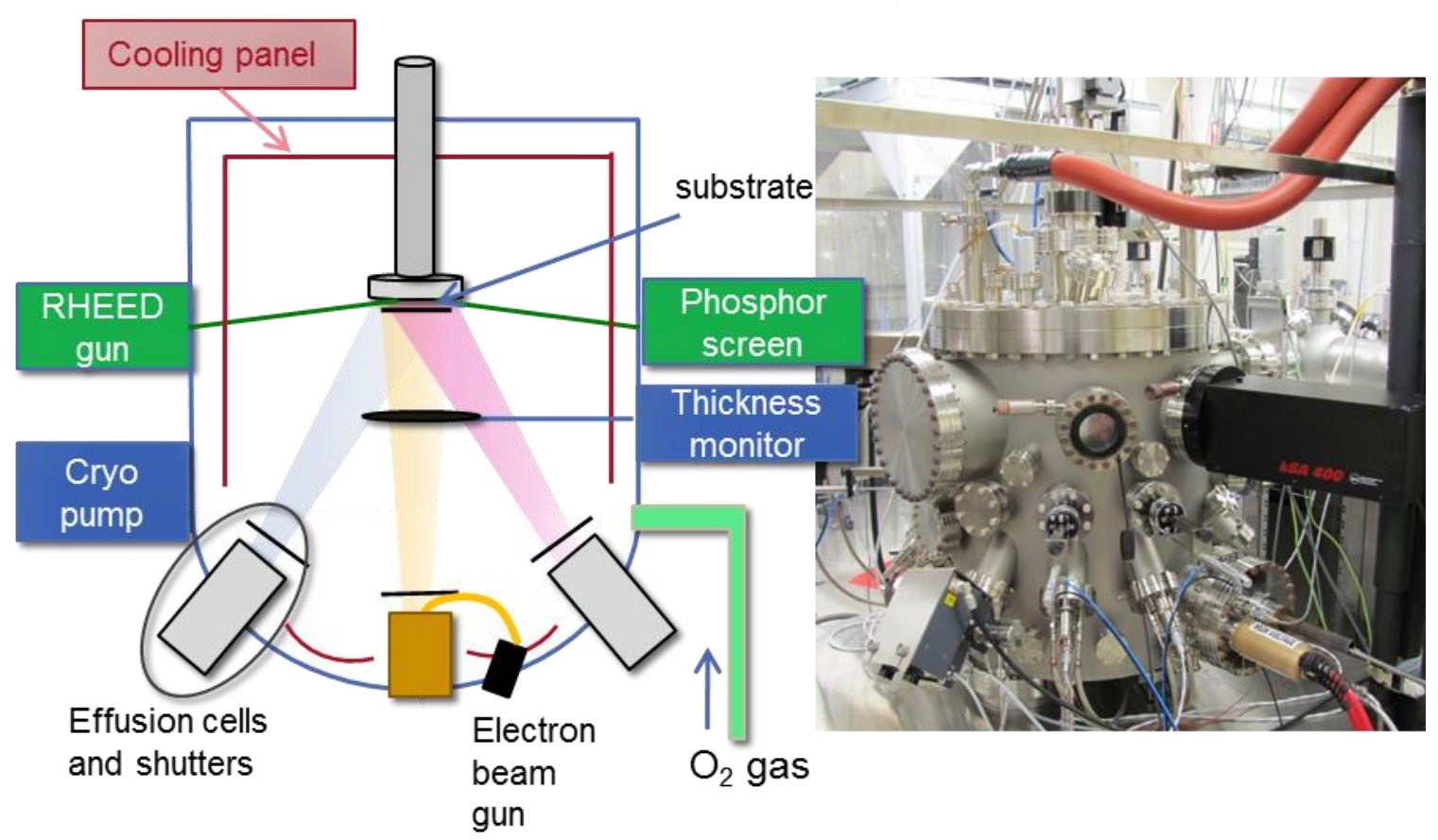

Figure 2.1: Schematic (left) and picture (right) of customized oxide molecular beam epitaxy chamber in the Materials Physics Lab at UT Austin. Labels indicate include components in the top-half (cooling panel, substrate, RHEED gun, phosphor screen, thickness monitor) and bottom-half (cryopump, effusion cells and shutters, electron beam gun, $\mathrm{O}_{2}$ gas) of the chamber.

Molecular beam epitaxy (MBE) is a powerful technique for atomic layer-by-layer deposition of thin films. Alternating deposition of molecules from sources in ultra-high vacuum (UHV) allows precise stoichiometry control. The technique was pioneered in the late 1960s and developed for III-V compounds in the 1970s [82]. In 1998, this technique was first applied to the uniquely challenging task of oxide heteroepitaxy on semiconductors [79]. Figure 2.1 displays the MBE chamber in the Materials Physics Lab 
at UT Austin. Main components include evaporation sources, gas sources, thickness monitor, and in situ reflection high-energy electron diffraction (RHEED).

Metal sources are often evaporated thermally from effusion cells (Figure 2.2) or, for the case of refractory metals with high melting points, evaporated by electron beam. High-temperature effusion cells can also be used to evaporate materials such as $\mathrm{Ti}$ and La from $\mathrm{TiC}$ and $\mathrm{W}$ crucibles, respectively. Background gas sources, such as oxygen and nitrogen, are introduced through a leak valve. For the case of MBE under oxygen-rich conditions, as in this dissertation, radiative heating of the substrate is much safer than direct heating.

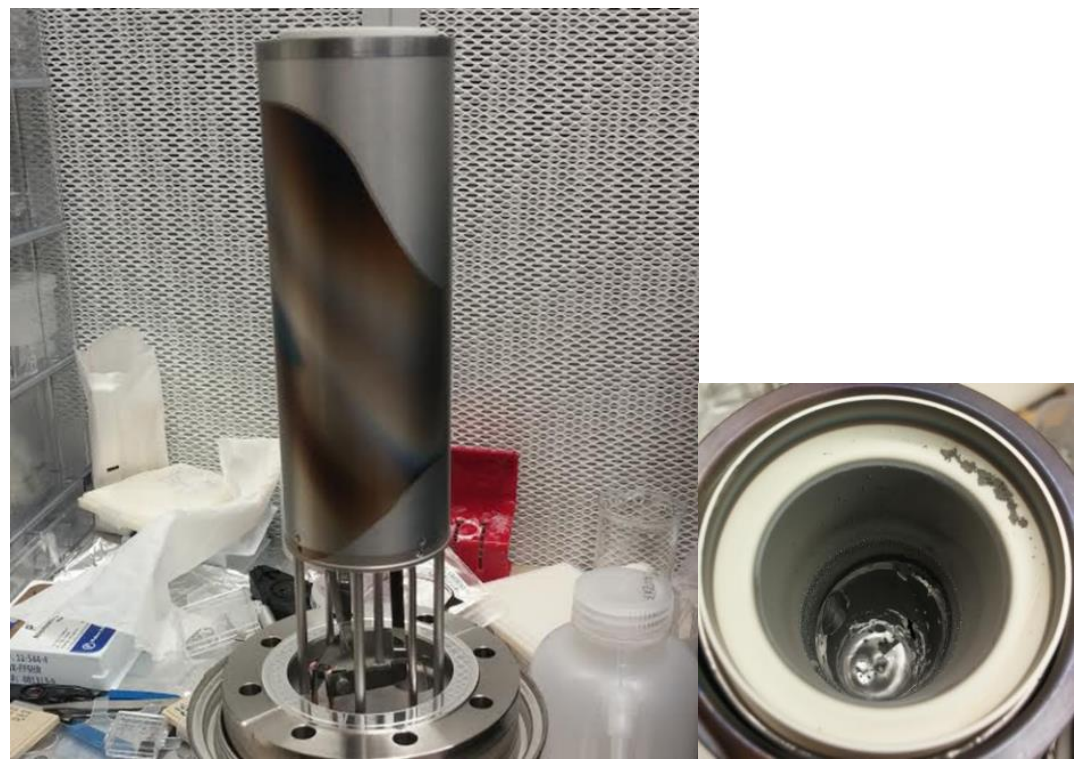

Figure 2.2: Left: Lowtemperature effusion cell. A knife-edge covered by a copper gasket in the bottom of the image is crucial to maintain UHV when the cell is mounted on the chamber pictured in Figure 2.1. Right: PBN crucible filled with metal in specialized cold-lipped effusion cell shows structural damage after repeated temperature cycling.

To form certain compounds, increased gas reactivity is desired to stay within a practical range of background gas pressures. An RF plasma generator can provide reactive gases such as nitrogen and oxygen. An ion deflector is employed to maximize reactive species while maintaining charge-neutrality. 
Quadrupole mass spectrometers, ion gauges, and quartz crystal monitors mounted in the chamber provide careful monitoring of the partial pressures and deposition rate. For a metal, such as $\mathrm{Sr}$, the target rate can be calibrated from the target surface number density per the equation,

$\rho \times N_{A} \times t / M=n_{2 D}$,

where $n_{2}$ is the target surface number density, $\rho$ and $M$ are the mass density and atomic weight of the metal, $N_{\mathrm{A}}$ is Avogadro's number, and $t$ is the thickness measured by the quartz crystal monitor. As a standard, rates can be calibrated using the $\operatorname{Si}(100)$ surface. The Si fcc unit cell has 2 atoms per $(5.43 \AA)^{2}$, or $n_{2 \mathrm{D}}=6.78 \times 10^{14} \mathrm{~cm}^{-2}$. Using Sr as an example $\left(M_{\mathrm{Sr}}=87.63 \mathrm{~g} / \mathrm{mol}, \rho_{\mathrm{Sr}}=2.63 \mathrm{~g} \cdot \mathrm{cm}^{-3}\right)$, using Eq. $(2.1)$ the target thickness for 1 ML $\mathrm{Sr}$ on $\mathrm{Si}(100)$ is $3.75 \AA$. Table 2.1 provides values for other metals used in this dissertation.

\begin{tabular}{ccccc}
\hline \hline Metal & Evaporation Source & $\begin{array}{c}\boldsymbol{M} \\
{[\mathbf{g} / \mathbf{M o l}]}\end{array}$ & $\left.\begin{array}{c}\boldsymbol{\rho} \\
{\left[\mathbf{g} \cdot \mathbf{c m}^{-2}\right.}\end{array}\right]$ & $\begin{array}{c}\boldsymbol{t} \text { for } \mathbf{S i}(\mathbf{1 0 0}) \\
{[\stackrel{\mathbf{A}}{]}]}\end{array}$ \\
\hline $\mathrm{Sr}$ & Low-T K-cell & 87.63 & 2.63 & 3.75 \\
$\mathrm{Co}$ & High-T K-cell OR & 58.93 & 8.90 & 0.75 \\
$\mathrm{Ti}$ & e-beam & 47.87 & 4.61 & 1.17 \\
$\mathrm{Al}$ & High-T K-cell & 26.98 & 2.70 & 1.13 \\
$\mathrm{Ba}$ & Lold-lipped low-T K-cell & 137.33 & 3.51 & 4.41 \\
$\mathrm{Eu}$ & Low-T K-cell & 151.96 & 5.26 & 3.25 \\
\hline \hline
\end{tabular}

Table 2.1: Evaporation rate calibration parameters for metals used in this dissertation.

As labelled in Figure 2.1, the chamber used for this dissertation was equipped with in situ RHEED, a powerful tool commonly used to characterize and monitor surface crystallinity [83] The high-energy electron beam scatters coherently from a periodic surface, and the resulting diffraction pattern is visible on the phosphor screen. The surface-sensitivity of RHEED stems from the glancing angle of incidence of the electron 
beam (less than $6^{\circ}$ ). The use of in situ RHEED system (STAIB) during the deposition is a significant advantage of the MBE growth method. In addition to symmetry monitoring, the oscillations of RHEED maxima intensity (Figure 2.3) can serve as a direct measure of the deposition rate. A local maximum indicates that a layer has been completed, while a local minimum indicates a half-completed layer.

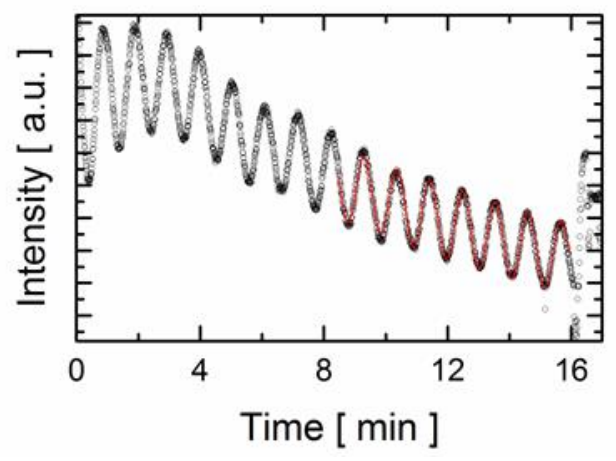

Figure 2.3: RHEED intensity oscillations for a fixed azimuth during STO deposition on $\mathrm{Si}(001)$. Each individual oscillation indicates the completion of one atomic layer of STO. From a sinusoidal fit to the data (red), the directly measured time for deposition of one atomic layer is approximately 1 minute and 4 seconds for this sample.

\subsection{X-RAY Photoelectron SPECTROSCOPY}

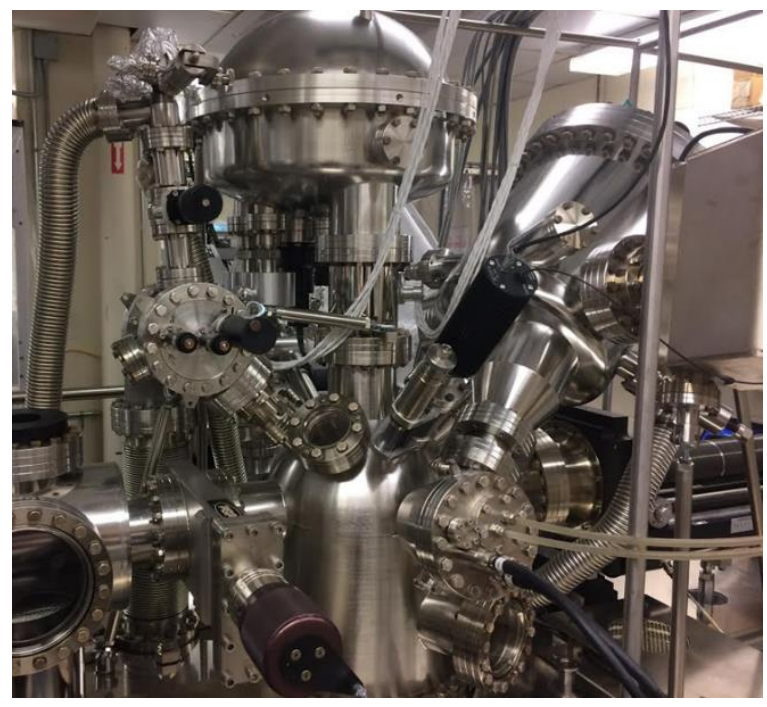

Figure 2.4: XPS analysis chamber in the Materials Physics Lab at UT Austin.

Another powerful surface science technique is $\mathrm{x}$-ray photoelectron spectroscopy (XPS). In the Materials Physics Lab at UT Austin, the deposition chamber (Figure 2.2) is connected to the VG Scienta R3000 photoemission chamber (Figure 2.4) by a twelve-foot-long UHV transfer line. This in situ transfer allows for incremental analysis during the film deposition without exposing the sample to ambient, and is particularly useful for oxygen- 
sensitive experiments. Available wavelengths include monochromatic Al Ka radiation $(\mathrm{h} v=1486.6 \mathrm{eV})$ and a UV $(\mathrm{h} v=21.22 \mathrm{eV})$ plasma-based source.

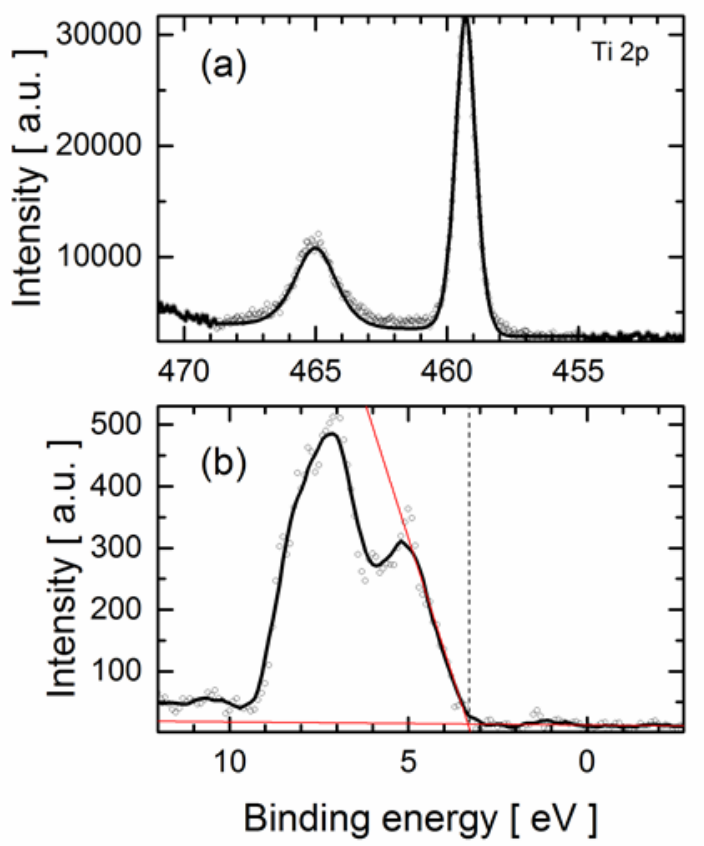

Figure 2.5: Ti $2 p$ (a) and valence band (b) spectrum of $\mathrm{Nb}$-doped $\mathrm{SrTiO}_{3}(100)$ substrate after a water boiling process and vacuum annealing [84].

This system allows for measurement of both core level and valence band spectra of the samples. For example, Figure 2.5 displays a typical $\mathrm{Ti}$ $2 p$ and valence band spectrum of $\mathrm{Nb}$ doped $\mathrm{SrTiO}_{3}(100)$ substrate after a water boiling process and vacuum annealing. [84] The procedure for core level and valence band fitting for oxide films is covered in greater detail in Chapters 3-7.

\subsection{FREe-SPACE ELECTRO-OPTIC CHARACTERIZATION}

We determined the Pockels coefficients by analyzing the change of the polarization of a laser beam transmitted through the BTO film while applying an electric field. To generate such a field, pairs of 300-nm-thick tungsten electrodes with the electrode gap $d=5 \mu \mathrm{m}$ were defined by optical lithography and $\mathrm{SF}_{6} / \mathrm{N}_{2}$ reactive ion etching. To assess the tensorial nature of the Pockels effect, we varied the angle $\theta_{\mathrm{E}}$ between the electric field and the BTO crystalline axis by fabricating differently oriented electrode pairs, as defined in Figure 2.6(a). 


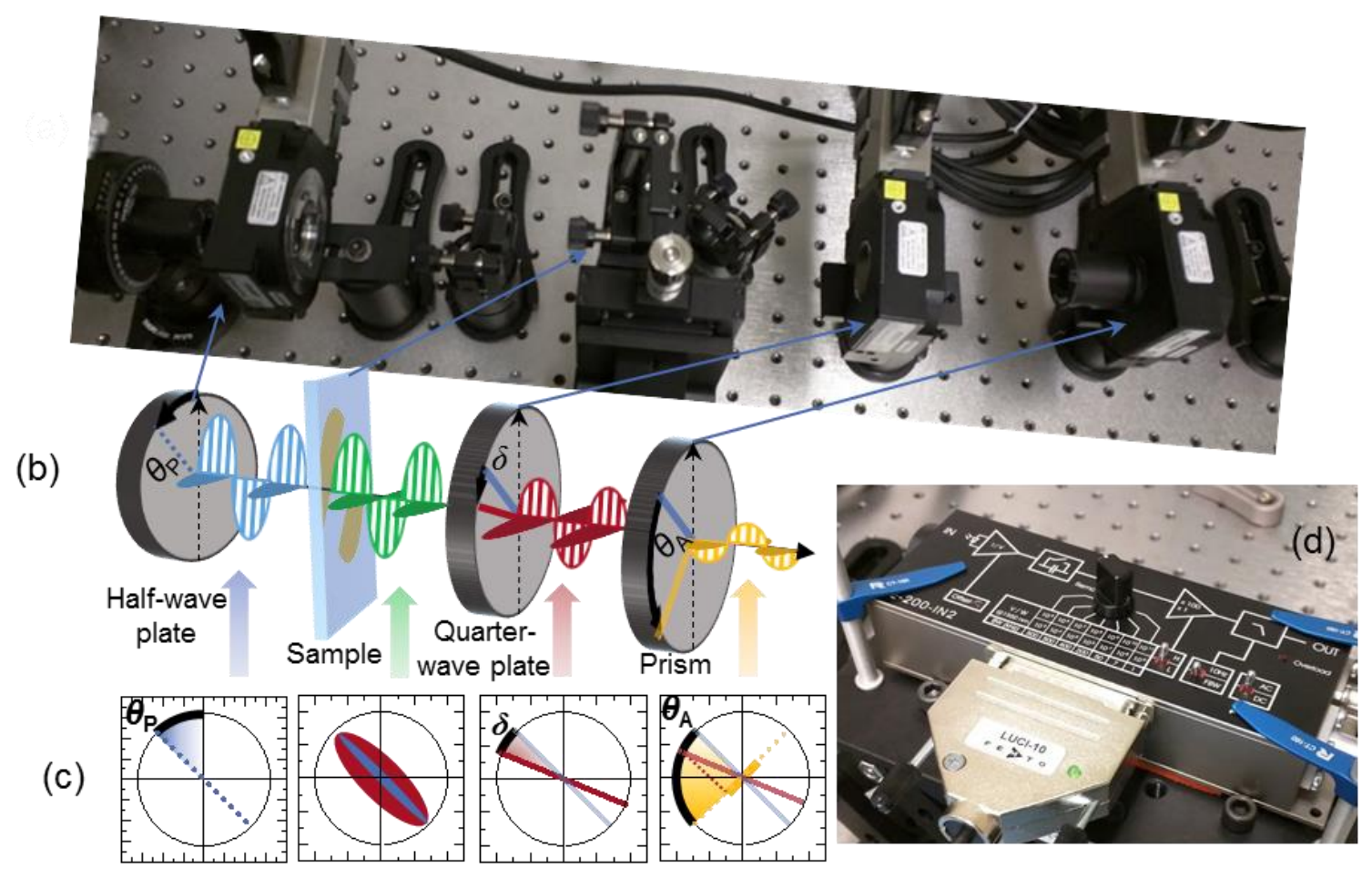

Figure 2.6: (a) Picture and (b) schematic of Sénarmont setup components. A half-wave plate is used to set the incident linear polarization. After passing through the sample, the light becomes elliptically polarized, which is transformed into linearly polarized light after passing a quarter-wave plate. Finally, a Glanlaser prism is used to determine the orientation of the transmitted polarization. (c) Corresponding states of the polarization along the path of the laser beam are visualized as the ellipse traced out by the light's electric field vector with (red) and without (blue) an external electric field. After transmission through the sample and quarter-wave plate, the polarization is rotated by angle $\delta$. (d) Detector for recording changes in transmission.

The electro-optic characterization follows the principle illustrated in Figure 2.6(b)-(c) using the Sénarmont set up described in detail elsewhere. [67] A linearly polarized New Focus diode laser model 6262 with wavelength $1550 \mathrm{~nm}$ focused to a spot size of $\sim 30 \mu \mathrm{m}$ full-width at half-maximum was first aligned to the electrode gap. A halfwave plate was used to set the incident polarization. Due to the birefringence of BTO, the 
sample introduces a phase shift between orthogonal polarization components of the incident light.

For increased sensitivity, a lock-in amplifier system was used to isolate the rotation $\delta$ induced by a sinusoidal AC field modulated at frequency $f=17.3 \mathrm{kHz}$. Typical values of the peak-to-peak AC voltage $V_{\mathrm{PP}} \sim 3 \mathrm{~V}$ were much smaller than the typical voltage used to align ferroelectric domains $V_{\mathrm{DC}} \sim 20 \mathrm{~V}$. Since in the lock-in configuration only the rotation $\delta$ corresponding to the AC modulation is measured, we introduce a field-normalized rotation, $\delta^{\prime}=\delta / E_{A C}$, where the root mean square AC field was defined as $E_{A C}=\mathrm{V}_{\mathrm{PP}} /(2 \sqrt{2} d)$ and $\delta$ is the rotation defined in Figure 2.6.

The static offset field applied to align ferroelectric domains was defined as $E_{\mathrm{off}}=V_{\mathrm{DC}} / d$, where $d$ is the gap between electrodes. While the magnitude of this offset field does not enter directly into the definition of the Pockels coefficient, it does influence the fraction of poled domains $v$. In the presence of polarization-reversed domains as in an un-poled film, a reduced response could be measured due to cancellation of contributions from antiparallel domains, leading to underestimated Pockels coefficients. [85] For this reason, Pockels coefficients reported in this dissertation are for poled films, corresponding to $v=1$ at at $\theta_{\mathrm{E}} \sim 45^{\circ}$ and $v=0.5$ at at $\theta_{\mathrm{E}} \sim 90^{\circ}$ as justified in the following sections.

The effective electro-optic coefficient can then be defined as follows, $r_{e f f}=\Gamma \lambda /\left(v \times E_{A C} \times \pi \times n_{B T O}^{3} \times t\right)$,

adapted from Ref. [86], where $\Gamma=2 \delta$ is the induced phase shift between orthogonal polarization components, $\lambda=1550 \mathrm{~nm}$ is the wavelength of the transmitted light, $v$ is the net fraction of poled BTO domains, $E_{\mathrm{AC}}$ is the measuring field defined above, $n_{\mathrm{BTO}}$ is the refractive index of the film, and $t$ is the thickness of the BTO layer. As described in detail elsewhere, [67] the rotation of polarization $\delta$ depends strongly on the orientation of the 
electric field. To capture this dependence and fully describe the Pockels effect, we report both the effective Pockels coefficient $r_{\text {eff }}$ and the $c$-axis Pockels coefficient $r_{\mathrm{c}}$ for each sample. Specifically, we extract $r_{\text {eff }}$ from measurements at $\theta_{\mathrm{E}} \sim 45^{\circ}$ assuming $v=1$ and $r_{\mathrm{c}}$ from measurements at $\theta_{\mathrm{E}} \sim 90^{\circ}$ assuming $v=0.5$. [67] Full dependence of these coefficients on the Pockels tensor elements are discussed in Refs. [67] and [85].

To extract Pockels tensor elements in addition to these effective values, numerical simulations based on the Pockels tensor and domain structure were carried out to best approximate $\delta$ for all measured electrode angles $\theta_{\mathrm{E}}$. The change in refractive index can be described by the change in the impermeability tensor,

$\Delta\left(\frac{1}{n^{2}}\right)_{i j}=\Delta \eta_{i j}=\sum_{k=1}^{3} r_{i j k} E_{k}$.

By symmetry using tensor notation the index ellipsoid can be represented in three dimensions,

$\frac{n_{x}^{2}}{n_{1}^{2}}+\frac{n_{y}^{2}}{n_{2}^{2}}+\frac{n_{z}^{2}}{n_{3}^{2}}+\frac{2 n_{x} n_{y}}{n_{6}^{2}}+\frac{2 n_{x} n_{z}}{n_{5}^{2}}+\frac{2 n_{y} n_{z}}{n_{4}^{2}}=1$

representing in general a refractive index ellipsoid which is in general distorted and rotated with respect to the zero-field values. By taking the projection of this index ellipsoid in the plane perpendicular to the angle of incidence passing through the center of the ellipsoid, we can extract the birefringence $n_{\mathrm{b}}=n_{\mathrm{o}}-n_{\mathrm{eo}}$ and rotation of optical axis $\theta_{\gamma}$. At this point, we must consider the ferroelectric domain structure, as described in detail in Appendix B, to determine a weighting factor for summing Jones vectors from each of the four distinct domain orientations. 


\title{
Chapter 3: Epitaxy of polar semiconductor $\mathrm{Co}_{3} \mathrm{O}_{4}(110)$
}

\author{
Contents of this Chapter were published in J. Appl. Phys. 115, 243708
} (2014) [87]. $\cdot^{\dagger}$

The (110) plane of $\mathrm{Co}_{3} \mathrm{O}_{4}$ spinel exhibits significantly higher rates of carbon monoxide conversion due to the presence of active $\mathrm{Co}^{3+}$ species at the surface. However, experimental studies of $\mathrm{Co}_{3} \mathrm{O}_{4}$ (110) surfaces and interfaces have been limited by the difficulties in growing high-quality films. We report thin (10-250 $\AA$ ) $\mathrm{Co}_{3} \mathrm{O}_{4}$ films grown by molecular beam epitaxy in the polar (110) direction on $\mathrm{MgAl}_{2} \mathrm{O}_{4}$ substrates. Reflection high-energy electron diffraction, atomic force microscopy, x-ray diffraction, and transmission electron microscopy measurements attest to the high quality of the asgrown films. Furthermore, we investigate the electronic structure of this material by core level and valence band x-ray photoelectron spectroscopy, and first-principles density functional theory calculations. Ellipsometry reveals a direct band gap of $0.75 \mathrm{eV}$ and other interband transitions at higher energies. A valence band offset of $3.2 \mathrm{eV}$ is measured for the $\mathrm{Co}_{3} \mathrm{O}_{4} / \mathrm{MgAl}_{2} \mathrm{O}_{4}$ heterostructure. Magnetic measurements show the signature of antiferromagnetic ordering at $49 \mathrm{~K}$. FTIR ellipsometry finds three infraredactive phonons between 300 and $700 \mathrm{~cm}^{-1}$.

\subsection{INTRODUCTION}

Complex oxides of cobalt find applications in gas sensing, [88,89] spintronics, [90-93] batteries, [89,91] and catalysis [94-101], most notably for oxidation of carbon monoxide (CO) $[96,97]$ and water. [98-101] At room temperature, the most

\footnotetext{
$\dagger$ Contributions: KJK designed and performed the experiments related to the sample growth, RHEED, $\mathrm{AFM}, \mathrm{XRD}$, and XPS and analyzed the data. AAD and ABP contributed to the conception and analysis of data. AS designed and performed first-principles calculations. AD and DJS performed TEM experiments. KNM, TIW, and SZ performed ellipsometry characterization. LGM and JZ performed and analyzed magnetic measurements. All authors contributed to interpretation of the data and to discussions.
} 
stable form of cobalt oxide is $\mathrm{Co}_{3} \mathrm{O}_{4}$, a semiconductor with a modest band gap of less than $2.0 \mathrm{eV}$, as compared to the $6.0 \mathrm{eV}$ gap of charge-transfer insulator $\mathrm{CoO}$. [102-107]

$\mathrm{Co}_{3} \mathrm{O}_{4}$ assumes the cubic $\mathrm{Fd} 3 m$ spinel-type phase, shown in Figure 3.1. Cobalt spinels have a history of use as gemstones, and their high stability makes them wellsuited for the decorating of ceramics (cobalt-containing spinels in particular are responsible for the striking "cobalt blue" pigment). [108,109] In $\mathrm{Co}_{3} \mathrm{O}_{4}$, vertices-sharing tetrahedral $\mathrm{Co}^{2+}$ ions carry a magnetic moment, while the nonmagnetic $\mathrm{Co}^{3+}$ ions occupy the edge-sharing octahedral sites. $\mathrm{Co}_{3} \mathrm{O}_{4}$ undergoes an antiferromagnetic phase transition below $\sim 40 \mathrm{~K} .[110-113]$

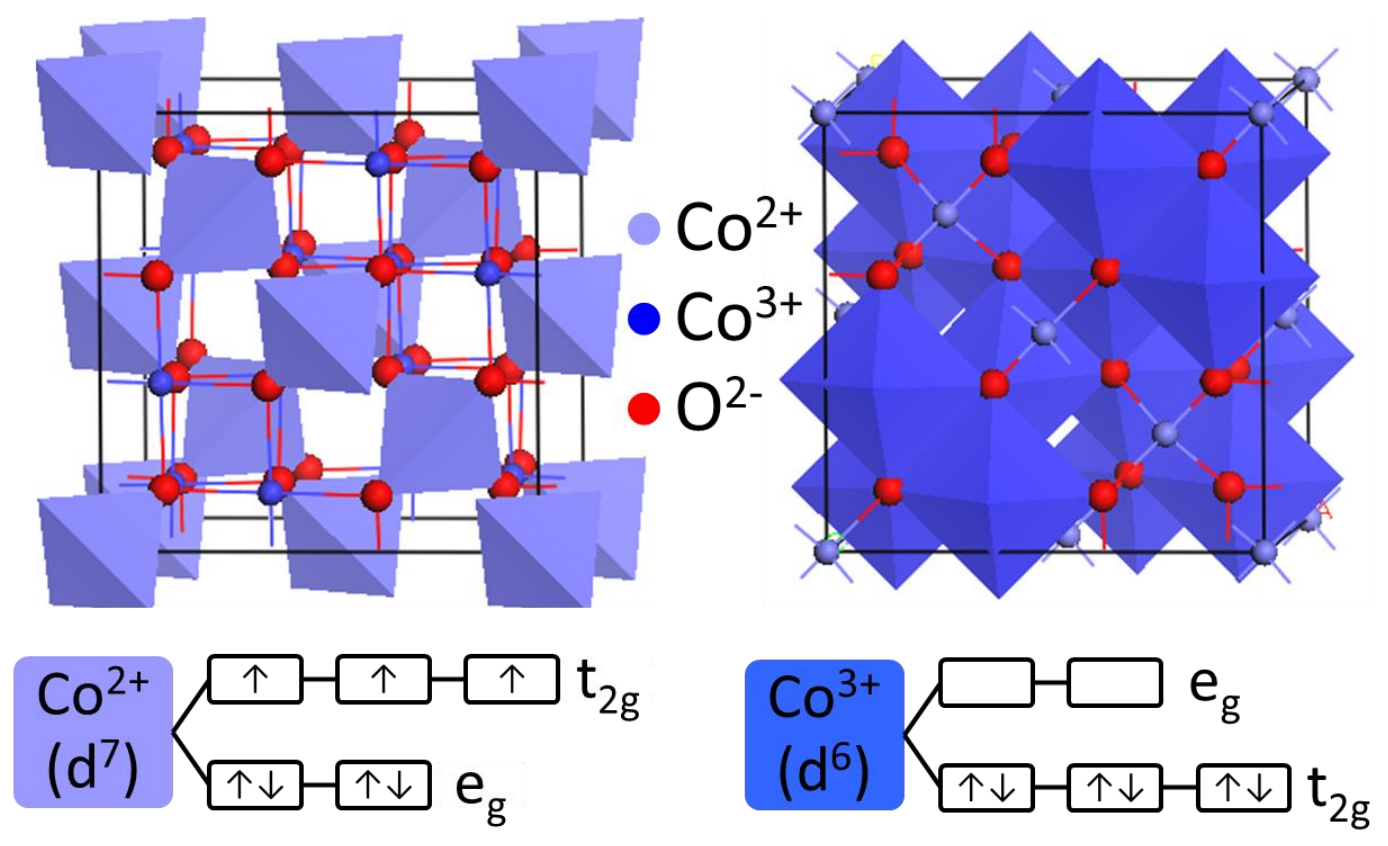

Figure 3.1: At room temperature, $\mathrm{Co}_{3} \mathrm{O}_{4}$ assumes the normal spinel structure. Based on the crystal field splitting, the $\mathrm{Co}^{2+}$ ions (tetrahedral, light blue) carry magnetic moment, while the $\mathrm{Co}^{3+}$ (octahedral, dark blue) ions are nonmagnetic. 
Studies of crystalline thin film surfaces and interfaces of this material could help provide insight into both its basic properties and catalytic behavior. [89,94-100] Of particular interest is the (110) plane that exhibits significantly higher reaction rates due to the presence of active $\mathrm{Co}^{3+}$ species at the surface [97]. Though epitaxially grown films offer a natural way to achieve high crystallinity, epitaxy of $\mathrm{Co}_{3} \mathrm{O}_{4}$ is complicated by the fact that all low-index planes are polar. As shown in Figure 3.2, the (110) direction of $\mathrm{Co}_{3} \mathrm{O}_{4}$ contains two types of alternating planes: the most stable type-A plane is comprised of units of $\mathrm{Co}_{2}^{3+} \mathrm{Co}_{2}^{2+} \mathrm{O}_{4}$ with a formal charge of +2 , while the type-B planes include $\mathrm{Co}_{2}^{3+} \mathrm{O}_{4}$, resulting in a charge of -2 per surface unit cell. This polarity further motivates study of polar $\mathrm{Co}_{3} \mathrm{O}_{4}$, as polar oxides are of prominent interest due to both their increased surface reactivity as compared to the bulk $[114,115]$ and as a mechanism for the formation of the two dimensional electron gas (2DEG). [35,116]

$\mathrm{Co}_{3} \mathrm{O}_{4}$ films have been grown by post-oxidation [104,117], atomic layer deposition (ALD), [118-120] chemical vapor deposition (CVD), [121-124] pulsed laser deposition (PLD), [105,125] and molecular beam epitaxy (MBE) [126-128]. A number of substrates, including $\mathrm{MgO},[105,120,122,124,125] \quad \mathrm{SrTiO}_{3},[105,120,124,125]$ $\mathrm{MgAl}_{2} \mathrm{O}_{4},[122,127,128] \mathrm{Al}_{2} \mathrm{O}_{3},[105,126] \mathrm{LaAlO}_{3}$, [125] Yttria-stabilized zirconia, [125] $\mathrm{SiO}_{2} / \mathrm{Si}$, [118,119] and Iridium [117] have been studied for the growth of crystalline $\mathrm{Co}_{3} \mathrm{O}_{4}$ films. Among these substrates, spinel $\mathrm{MgAl}_{2} \mathrm{O}_{4}$ (110) is an ideal substrate choice not only due to its small lattice mismatch with $\mathrm{Co}_{3} \mathrm{O}_{4}$ (less than $0.05 \%$ ), but also due to the fact that it is the only substrate thus far on which $\mathrm{Co}_{3} \mathrm{O}_{4}$ preferentially grows (110)oriented. [127,128] MBE growth offers several advantages including precise control over flux and in situ analysis, but reports by Vaz et al. have suggested that extensive postgrowth ex situ annealing is required. [127,128] 

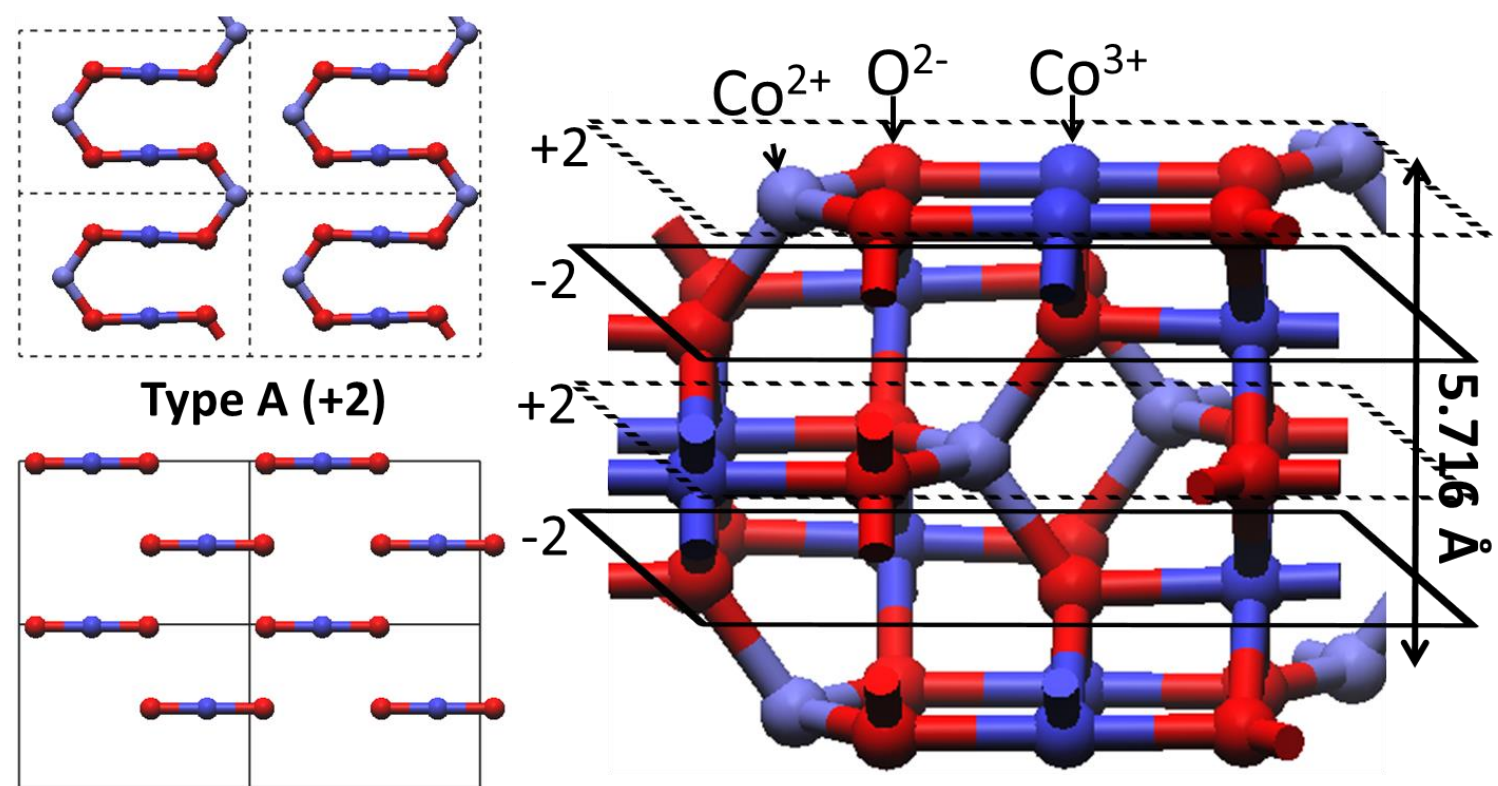

Type B (-2)

Figure 3.2: Side view: the (110) direction of $\mathrm{Co}_{3} \mathrm{O}_{4}$ is characterized by a 4-repeat period over a depth of $5.7 \AA$. The alternating planes are shown from a top view. The type A plane is comprised of units of $\mathrm{Co}^{3+}{ }_{2} \mathrm{Co}^{2+}{ }_{2} \mathrm{O}_{4}$ with a formal charge of +2 , while the type $\mathrm{B}$ planes include $\mathrm{Co}^{3+}{ }_{2} \mathrm{O}_{4}$ resulting in a charge of -2 .

In this chapter, we report on thin $\mathrm{MBE}$-grown $\mathrm{Co}_{3} \mathrm{O}_{4}$ films on $\mathrm{MgAl}_{2} \mathrm{O}_{4}$ substrates. We probe the structure of these as-grown highly crystalline (110)-oriented films by reflection high-energy electron diffraction (RHEED), atomic force microscopy (AFM), x-ray diffraction (XRD), and transmission electron microscopy (TEM). The film surface roughens at intermediate thickness, but slowly smoothens as growth continues, returning to an RMS surface roughness of $\sim 1 \AA$. A valence band offset of $3.2 \mathrm{eV}$ is measured for the heterostructure by x-ray photoelectron spectroscopy (XPS). Firstprinciples density functional theory (DFT) calculations matching the shape of the XPS valence band calculate a band gap of $0.6 \mathrm{eV}$ for $\mathrm{Co}_{3} \mathrm{O}_{4}$. Ellipsometry reveals a direct band gap of $0.75 \mathrm{eV}$ and other interband transitions at higher energies. FTIR ellipsometry 
finds three infrared-active phonons between 300 and $700 \mathrm{~cm}^{-1}$. Superconducting quantum interference device (SQUID) magnetometry measurements show the signature of antiferromagnetic ordering at an enhanced Néel temperature of $49 \mathrm{~K}$.

\subsection{EXPERIMENTAL DETAILS}

$\mathrm{MgAl}_{2} \mathrm{O}_{4}$ substrates of dimension $5 \mathrm{~mm} \times 5 \mathrm{~mm} \times 0.5 \mathrm{~mm}$ were degreased in acetone, isopropanol, deionized water, and UV ozone. Substrates were glued with silver paste to a sample holder to ensure good thermal contact. The samples were then introduced into a customized DCA $600 \mathrm{MBE}$ system with a base pressure of $3 \times 10^{-10}$ Torr. All substrates were outgassed in the chamber at $700^{\circ} \mathrm{C}$ for 30 minutes before lowering the substrate temperature to $500^{\circ} \mathrm{C}$ for growth. The substrate temperature is measured by a thermocouple in close proximity to the substrate heater and that the thermocouple is calibrated using a pyrometer measurement of a silicon substrate.

The electron-beam evaporated Co flux was calibrated to a deposition rate of 3-4 $\AA /$ minute by use of a quartz crystal microbalance. Atomic oxygen was then introduced by means of an RF plasma source at a power of $250 \mathrm{~W}$ with an oxygen background pressure of $1 \times 10^{-5}$ Torr. After growth, the main shutter was closed, and the samples were cooled down in the presence of oxygen. The presence of the film affects the visual appearance of the sample, adding a brown tint as compared to the transparent substrate. This is consistent with the smaller band gap of $\mathrm{Co}_{3} \mathrm{O}_{4}$ as compared to that of the $\mathrm{MgAl}_{2} \mathrm{O}_{4}$ substrate. [105,129] For ellipsometry measurements, a $\mathrm{Co}_{3} \mathrm{O}_{4}$ film was deposited on a large 2"-diameter $\mathrm{MgAl}_{2} \mathrm{O}_{4}$ substrate to eliminate artifacts due to the size of the sample. The unpolished side of this wafer was coated with $\mathrm{Nb}$ metal rather than silver paste before following the steps outlined above. 
The samples were monitored during growth by in situ RHEED, and then transferred in situ to the XPS analysis chamber (VG Scienta R3000). For the remaining characterization techniques (AFM, XRD, TEM, Ellipsometry, and SQUID), the samples were removed from UHV and exposed to atmosphere.

Spectroscopic ellipsometry measures the complex Fresnel ratio

$$
\rho=\frac{r_{p}}{r_{s}}=\tan \psi e^{i \Delta}
$$

i.e., the ratio of the p-polarized reflectance $r_{p}$ to the s-polarized reflectance $r_{s} . \rho$ is usually expressed in terms of the ellipsometric angles $\psi$ and $\Delta$. For a bulk sample with complex refractive index $n, \rho$ is given by $[130,131]$

$$
\rho=\frac{\left(n \cos \phi_{0}-\cos \phi_{1}\right)\left(\cos \phi_{0}+n \cos \phi_{1}\right)}{\left(n \cos \phi_{0}+\cos \phi_{1}\right)\left(\cos \phi_{0}-n \cos \phi_{1}\right)}
$$

where $\phi_{0}$ is the angle of incidence and $\phi_{1}$ the angle of refraction given by Snell's Law.

The imaginary part of the dielectric function $\varepsilon=n^{2}$ is related to absorption in the sample by elementary excitations. For example, the absorption in the infrared (IR) spectral region is given by lattice absorption of infrared-active phonons or the free-carrier response in a metal. In the visible and UV spectral region, the absorption is due to interband electronic transitions. The application of this technique to bulk spinel $\left(\mathrm{MgAl}_{2} \mathrm{O}_{4}\right)$ has been described recently. [129]

In the case of a thin film on a substrate, we can still invert Eq. (3.1) to calculate $\langle n\rangle_{\text {and }}\langle\varepsilon\rangle=\langle n\rangle^{2}$, but these quantities are no longer related to the optical constants of a specific material. Instead, $\langle n\rangle$ and $\langle\varepsilon\rangle$ are determined by the optical constants of the film and the substrate along with the film thickness and surface and interface roughness layer thicknesses. Therefore, $\langle n\rangle$ and $\langle\varepsilon\rangle$ are called the pseudo-refractive index and the pseudo-dielectric function. If the dielectric function $\varepsilon_{s}$ of the substrate and the film 
thickness are known, then the dielectric function $\varepsilon_{f}$ of the film can be determined by fitting the experimental data using well-established procedures [130,131] and commercial software (J.A. Woollam Co., Inc., WVASE32). Surface roughness can also be considered within this approach.

For this work, we used two instruments to acquire our data. From 0.76 to $6.5 \mathrm{eV}$, we measured the ellipsometric angles on a Woollam variable angle-of-incidence spectroscopic ellipsometer (VASE) with computer-controlled Berek waveplate compensator at seven angles of incidence $\left(50^{\circ}\right.$ to $\left.80^{\circ}\right)$. For IR ellipsometry, we used a Woollam FTIR-VASE ellipsometer, which is based on a fixed analyzer $\left(0^{\circ}\right.$ and $\left.180^{\circ}\right)$ and polarizer $\left( \pm 45^{\circ}\right)$ and a rotating compensator. We measured from 250 to $7000 \mathrm{~cm}^{-1}$ at five angles of incidence $\left(60^{\circ}\right.$ to $\left.80^{\circ}\right)$. In the FTIR experiments, the spectral resolution was set to $8 \mathrm{~cm}^{-1}$ to improve the signal to noise ratio. All data sets were merged and fitted simultaneously with the same model, including both lattice absorption and interband electronic transitions. The optical constants for the $\mathrm{MgAl}_{2} \mathrm{O}_{4}$ substrate were taken from Ref. [129] without adjustments. We assumed a surface roughness of $2 \AA$, guided by atomic force microscopy measurements. In the case of a thin absorbing $\mathrm{Co}_{3} \mathrm{O}_{4}$ layer on a substrate, the layer thickness cannot be determined. We use a film thickness of $220 \AA$ (determined from the growth parameters and confirmed by x-ray reflectance measurements) as an input for our ellipsometry analysis. Small errors in film thickness cause errors in the amplitude of the dielectric function, but do not affect the peak energies and broadenings.

\subsection{RESUlTS AND DisCUSSION}

The spinel surface was characterized during film deposition using in situ RHEED (Figure 3.3). The most stable surface of the $\mathrm{MgAl}_{2} \mathrm{O}_{4}$ (110) substrate is the A-type, which 
contains both $\mathrm{Mg}$ and $\mathrm{Al}$ atoms. The RHEED pattern for the substrate (Figure 3.3(a)) confirms the crystalline quality of the surface, with sharp diffraction maxima as expected for a periodic surface with negligible roughness. As the $\mathrm{Co}_{3} \mathrm{O}_{4}$ film grows (Figure 3.3(b), (d)), it follows the termination of the substrate to preserve charge neutrality, as is common in polar materials. [114,127]

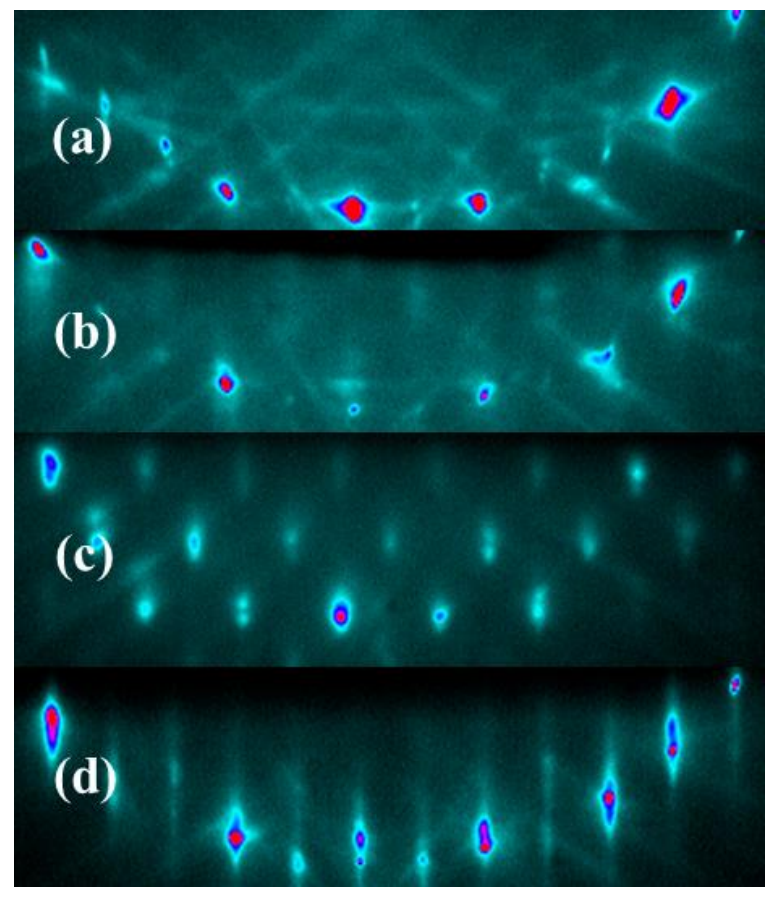

Figure 3.3: RHEED patterns along the [110] azimuth at grazing incidence for (a) the $\mathrm{MgAl}_{2} \mathrm{O}_{4}$ substrate, (b) $\sim 13$ - $\AA$-thick $\mathrm{Co}_{3} \mathrm{O}_{4}$ film, (c) $\sim 24-\AA$-thick film, (d) 125 $\AA$-thick film. The most stable surface of the $\mathrm{MgAl}_{2} \mathrm{O}_{4}$ substrate is the A-type, which contains both $\mathrm{Mg}$ and $\mathrm{Al}$ atoms. The $\mathrm{Co}_{3} \mathrm{O}_{4}$ film follows the termination of the substrate to preserve charge neutrality, as is common in polar materials. The surface develops roughness at thickness starting between 10-20 $\AA$, causing the observed broadening of the RHEED streaks. The surface again becomes smooth as growth continues, returning to substrate quality around $100 \AA$ thickness.

The surface roughens with thickness starting between 10-20 A, causing broadening of the RHEED streaks. However, as the film continues to grow, the surface again becomes smooth, with a crystalline quality approaching that of the substrate at around 100- $\AA$-thickness and above (Figure 3.3(d)). One possible explanation for these observations is the electrostatic energy of a polar material diverges with increasing thickness. [114,132] Above a critical field and therefore critical thickness, the surface roughness may develop to compensate the surface charge density. Once the film can instead compensate by bulk rather than surface reconstruction, the surface can once again become smooth, as seen in the thicker films. 
These RHEED observations are supported by ex situ AFM measurements for $\mathrm{Co}_{3} \mathrm{O}_{4}$ samples of varying thickness. Scans of $5 \times 5 \mu \mathrm{m}$ areas are shown for the substrate and three representative films in Figure 3.4. For the $\sim 13$ - $\AA$-thick film (Figure 3.3(b), Figure 3.4(b)), the RMS roughness was $1.26 \AA$. This is on the order of instrumental resolution; any perceived modulation of the surface is likely due to vibrations of the microscope. For slightly thicker films, such as the $\sim 24 \AA$ film (Figure 3.3(c), Figure 3.4(c)), the surface becomes noticeably rougher. In this sample, the RMS roughness increased to $5.26 \AA$. Finally, the $125 \AA$ film shown in Figure 3.4(d) shows the surface roughness has decreased back down to $1.04 \AA$; this sample serves as a representative example of the smoothing that occurs in thicker films.
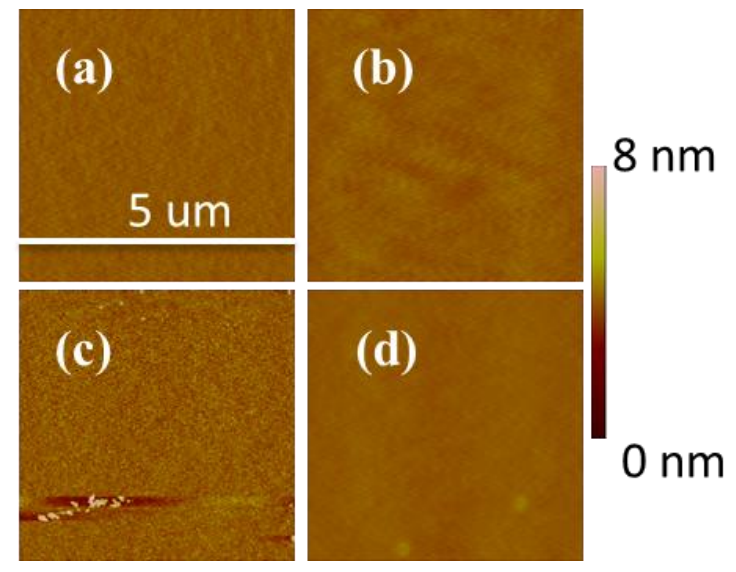

Figure 3.4: AFM measurements for: (a) bare $\mathrm{MgAl}_{2} \mathrm{O}_{4}$ substrate, followed by $\mathrm{Co}_{3} \mathrm{O}_{4}$ samples of thickness (b) $13 \AA$, (c) $24 \AA$, and (d) $125 \AA$. The RMS roughness measured was $1.52 \AA, 1.26 \AA, 5.26 \AA$, and $1.04 \AA$, respectively.

X-ray diffraction measurements were carried out using a Panalytical X'PERT Pro diffractometer $(\mathrm{Cu} \mathrm{K} \alpha 1$ source, $\lambda=1.5406 \AA$ ) operating at $45 \mathrm{kV}$ and $40 \mathrm{~mA}$. The symmetric $2 \Theta-\Theta$ scan shows strong substrate peaks corresponding to an out-of-plane lattice constant of $8.07 \AA$. The high-resolution rocking curve of the (440) reflection for a 240 - $\AA$-thick film $\mathrm{Co}_{3} \mathrm{O}_{4}$ is shown in Figure 3.5(a). Experimental data points are shown by open circles, and a sum of two Voigt functions has been fitted to the data (blue curve). The contribution from the substrate (red curve) is mostly Gaussian, with a width of $0.015^{\circ}$, on the order of instrumental resolution as expected for a single-crystal substrate. The film peak (green 
curve) has a width $0.03^{\circ}$, not much broader than that of the substrate. The shaded green area outlines values within the uncertainty of the position of the film peak, showing that the film is nearly lattice-matched to the substrate. Spot spacing in RHEED indicates that there is no change in the in-plane lattice constant for the film compared to the substrate up to the thickness that we have grown $(250 \AA)$.
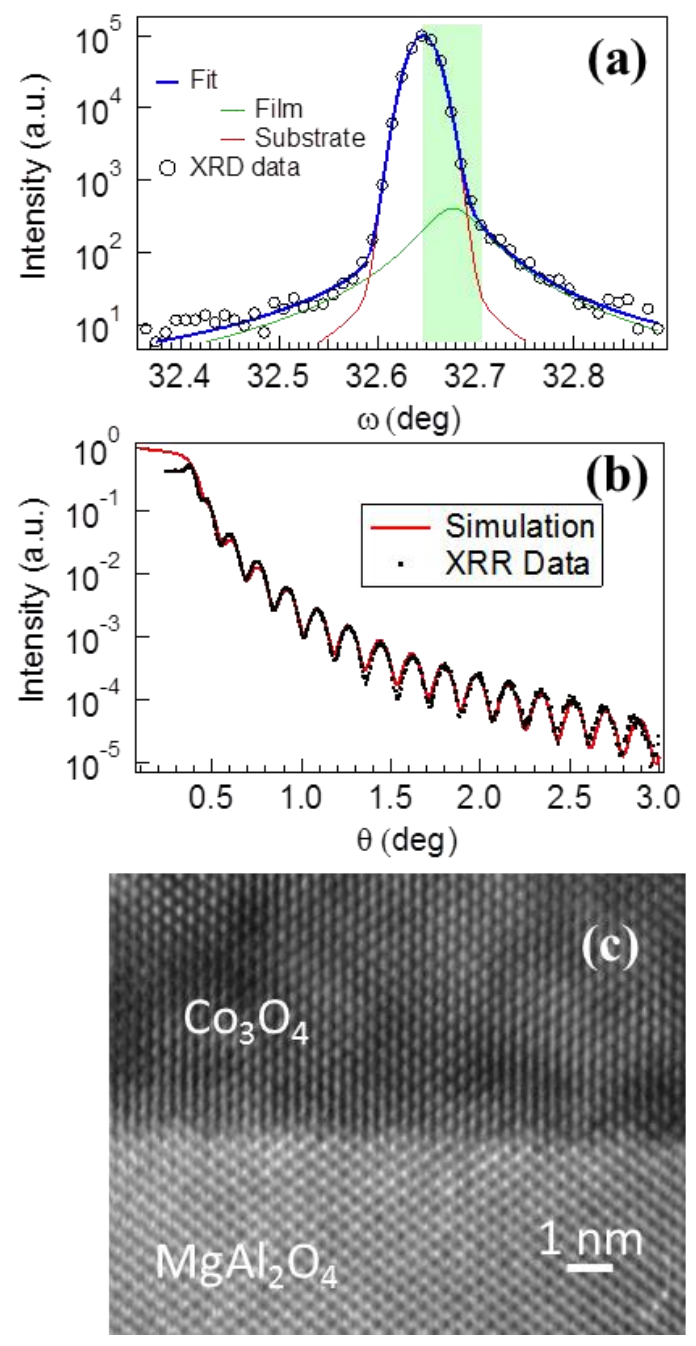

Figure 3.5: (a) Rocking curve data and analysis of the (440) reflection of $240 \AA$ film $\left(\mathrm{Co}_{3} \mathrm{O}_{4}\right.$ on $\left.\mathrm{MgAl}_{2} \mathrm{O}_{4}\right)$. Experimental data points are shown by open circles, and a sum of two Voigt functions has been fitted to the data. (b) X-ray reflectivity for the same film. The drawn (red) line is a simulation. Oscillations due to the difference in density between the substrate and film persist undamped to high angles, implying a smooth surface and abrupt interface. (c) High-resolution crosssectional transmission electron micrograph confirms the abrupt interface between the crystalline substrate and film.

All thicknesses referenced above were first estimated from the quartz crystal microbalance calibration and verified by X-ray reflectivity (XRR). In this geometry, oscillations in the reflected intensity arise due to the difference in density between the substrate and film. An example is

shown for the same $240 \AA$ film (Figure 3.5(b)); the black line is experimental data, while the drawn (red) line is a simulation. The simulated oscillations correspond to a film 
thickness of $240 \AA$ with $1 \AA$ roughness. Strong oscillations with minimal damping are indicative of a smooth surface and abrupt interface for the as-grown sample.

To further characterize the interface, Figure 3.5(c) shows a cross-sectional transmission electron micrograph taken with a JEOL JEM-4000EX transmission electron microscope operated at $400 \mathrm{keV}$. The image shows a highly crystalline epitaxial $\mathrm{Co}_{3} \mathrm{O}_{4}$ film on the $\mathrm{MgAl}_{2} \mathrm{O}_{4}$ substrate with a sharp and coherent interface, in agreement with the RHEED and XRD results above.
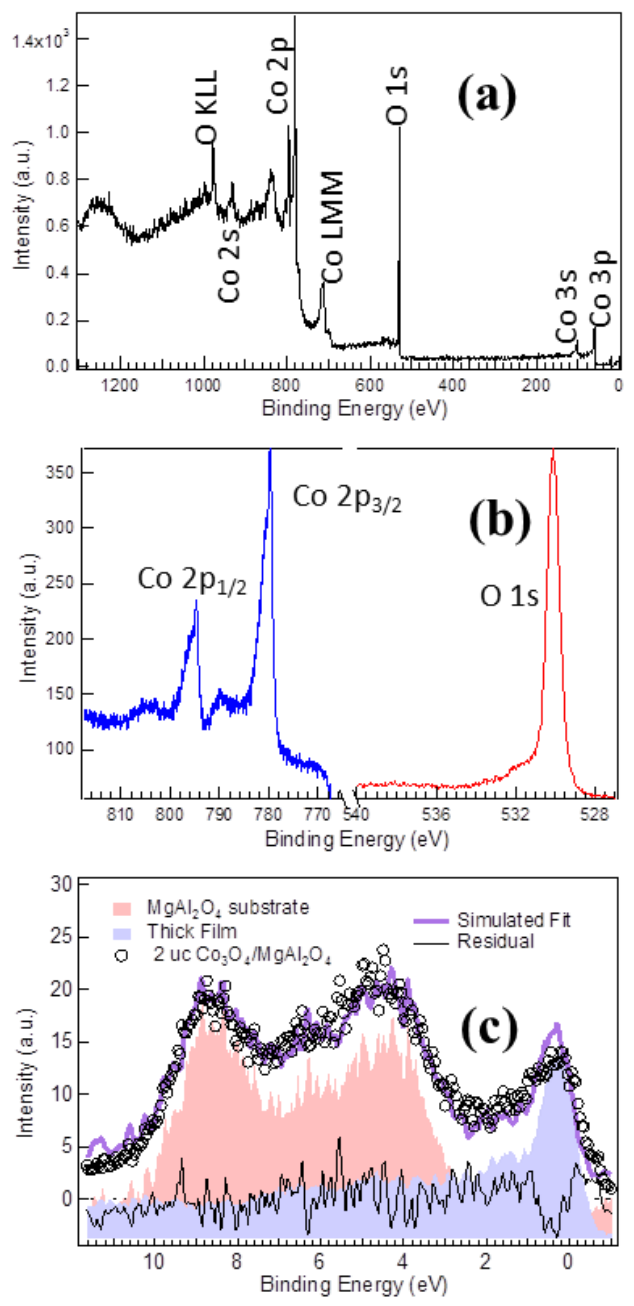

Figure 3.6: XPS spectra. (a) Survey scan of representative 250 - $\AA$-thick $\mathrm{Co}_{3} \mathrm{O}_{4}$ film shows a spectrum dominated by cobalt and oxygen. (b) High-resolution Co $2 p$ scan shows characteristic strongly suppressed shake-up satellite peaks at $+5.5 \mathrm{eV}$ and +9 $\mathrm{eV}$, as compared to $\mathrm{CoO}$. The $\mathrm{O} 1 \mathrm{~s}$ peak shows a slight shoulder, which can be attributed to surface oxygen. (c) To determine the valence band offset, the spectra for pure $\mathrm{MgAl}_{2} \mathrm{O}_{4}$ (red shading) and $\mathrm{Co}_{3} \mathrm{O}_{4}$ (blue shading) were scaled, offset, and added together to create a simulated fit (purple line) to the measured valence band spectra for a heterostructure of $13 \AA \mathrm{Co}_{3} \mathrm{O}_{4}$ on $\mathrm{MgAl}_{2} \mathrm{O}_{4}$ (black open circles). The residual difference is also plotted (black line).

XPS measurements were performed in situ using monochromatic Al $\mathrm{K} \alpha$ radiation $(\mathrm{h} v=1486.6 \mathrm{eV})$. To avoid charging due to the highly insulating substrate, a low-energy electron flood gun was used to replace the emitted electrons. Figure 3.6 summarizes the core level 
spectroscopy. A representative survey scan of a bulklike $250-\AA$-thick $\mathrm{Co}_{3} \mathrm{O}_{4}$ film shows the expected peaks of a system dominated by cobalt and oxygen (Figure 3.6(a)). Higherresolution scans of the O1s and Co $2 p$ peaks are shown in Figure 3.6(b). Spectra have been artificially shifted to fix the location of the O1s peak at $530.1 \mathrm{eV}$. The Co $2 \mathrm{p}$ scan shows characteristic strongly suppressed shake-up satellite peaks at $+5.5 \mathrm{eV}$ and $+9 \mathrm{eV}$ as compared to CoO. [102]

Figure 3.6(c) displays XPS valence band spectra for the bare $\mathrm{MgAl}_{2} \mathrm{O}_{4}$ substrate (red shading) and 250- $\AA$-thick $\mathrm{Co}_{3} \mathrm{O}_{4}$ film (blue shading). The band offset in $\mathrm{Co}_{3} \mathrm{O}_{4}$ heterostructures may facilitate understanding of the catalytic properties of $\mathrm{Co}_{3} \mathrm{O}_{4}$; for example, in order to achieve a spontaneous water splitting reaction, both a small band gap and suitable alignment of band edges with respect to the water redox potentials are required. Band offsets were calculated by both core-level [101] and valence band $[133,134]$ spectroscopy. The valence band maximum (Еvвм) for each material was calculated using the linear extrapolation method. [135] By comparing energy offsets between the Co 2p, $\mathrm{Al} 2 \mathrm{p}$, and valence band edge positions (as summarized in Table 3.1) using

$V B O_{C L}=\left(E_{C o 2 p}-E_{V B M}\right)_{C_{3} O_{4}}-\left(E_{A l 2 p}-E_{V B M}\right)_{M_{g A l} O_{4}}-\left(E_{C o 2 p}-E_{A l 2 p}\right)_{C_{3} O_{4} / M g A_{2} O_{4}}$, we calculate a $\mathrm{VBO}_{\mathrm{CL}}$ of $3.4 \mathrm{eV}$.

\begin{tabular}{cccc}
\hline \hline Materials & $\begin{array}{c}\mathrm{MgAl}_{2} \mathrm{O}_{4} \\
\text { substrate }\end{array}$ & $\begin{array}{c}\sim 13 \AA \\
\mathrm{Co}_{3} \mathrm{O}_{4} / \mathrm{MgAl}_{2} \mathrm{O}_{4}\end{array}$ & $\mathrm{Co}_{3} \mathrm{O}_{4}$ thick film \\
\hline $\begin{array}{c}\mathrm{Co} 2 p \\
(\mathrm{eV})\end{array}$ & -- & 777.02 & 777.87 \\
$\begin{array}{c}\mathrm{Al} 2 p \\
(\mathrm{eV})\end{array}$ & 74.40 & 74.65 & -- \\
$\begin{array}{l}\mathrm{VBM} \\
(\mathrm{eV})\end{array}$ & 3.17 & -- & 0.852 \\
\hline \hline
\end{tabular}

Table 3.1: XPS core levels for valence band offset calculation. 
The VBO can also be determined directly from the valence band spectra. $[133,134]$ For a heterostructure of $13 \AA \mathrm{Co}_{3} \mathrm{O}_{4} / \mathrm{MgAl}_{2} \mathrm{O}_{4}$ a contribution from the substrate is visible along with an attenuated contribution from the substrate (Figure 3.6(c), black open circles). We simulated a fit (purple solid line) to the heterostructure by scaling and offsetting the pure valence band spectra measured for the $\mathrm{MgAl}_{2} \mathrm{O}_{4}$ substrate and thick $\mathrm{Co}_{3} \mathrm{O}_{4}$ film. This method uses an "all at once" fit minimizing $\chi^{2}$ by the Levenberg-Marquardt algorithm implemented in Igor Pro software (WaveMetrics, Lake Oswego, OR). The difference between the measured and simulated spectra is also plotted (black line). Using this method, we calculate $\mathrm{VBO}_{\text {sim }}=3.2 \mathrm{eV}$, in good agreement with the offset calculated above by the core levels.

We modeled the electronic structure of bulk $\mathrm{Co}_{3} \mathrm{O}_{4}$ using the Hubbard-corrected local density approximation $(\mathrm{LDA}+\mathrm{U})$ to DFT. The problem is difficult, as two different oxidation states of Co with two different spin states must be described. Because of this, U-values for $\mathrm{Co}_{3} \mathrm{O}_{4}$ calculations vary widely in the literature. [105,136-138] The problem is further complicated by the debate over the size of the fundamental band gap of $\mathrm{Co}_{3} \mathrm{O}_{4}$.

(b)

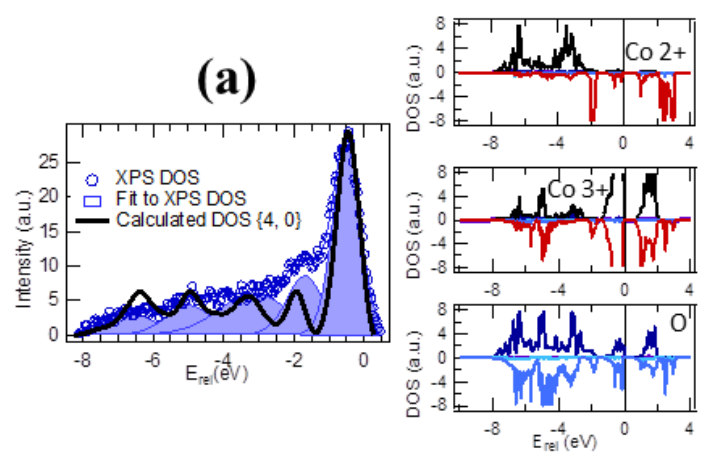

Figure 3.7: Valence band structure (a) Measured XPS valence band for a $250-\AA \AA-$ thick sample is shown by open circles, with the envelope of the 5-peak fit shaded blue. Indicated by the solid black line, calculated density of states (DOS) for bulk $\mathrm{Co}_{3} \mathrm{O}_{4}$ with $\mathrm{U}\left(\mathrm{Co}^{2+}\right)=4 \mathrm{eV}$ and $\mathrm{U}\left(\mathrm{Co}^{3+}\right)=$ $0 \mathrm{eV}$ agrees well with experimental features. (b) Site- and orbital-projected DOS for $\mathrm{U}\left(\mathrm{Co}^{2+}\right)=4 \mathrm{eV}$ and $\mathrm{U}\left(\mathrm{Co}^{3+}\right)=0$ $\mathrm{eV}$. 
which best matches experimental data, we started with the Shirley-background-subtracted XPS valence band (Figure 3.7(a), blue open circles) and fit 5 peaks to the data (Figure 3.7(a), blue shaded regions). 5 peaks was the minimum number necessary to acquire a reasonable fit. We then calculated the total density of states (DOS) for 64 combinations of $\mathrm{U}$ (from $0-7 \mathrm{eV}$ on $\mathrm{Co}^{2+}$ and $\mathrm{Co}^{3+}$ in $1 \mathrm{eV}$ increments), broadened each DOS by $0.5 \mathrm{eV}$ to account for experimental broadening, and again fit 5 peaks to each DOS. We then compared the overall band width and peak positions with the experimental data, narrowing down from the 64 original possible combinations to 3 best fits: $\left\{\mathrm{U}\left(\mathrm{Co}^{2+}\right)\right.$, $\left.\mathrm{U}\left(\mathrm{Co}^{3+}\right)\right\}=\{0,0\},\{0,3\}$, and $\{4,0\}$. We then checked the magnetization as function of the two $\mathrm{U}$ parameters. Combination $\{4,0\}$ produced a magnetization of $2.65 \mu_{\mathrm{B}}$ for the $\mathrm{Co}^{2+}$ ions, while $\{0,0\}$ and $\{3,0\}$ produce lower values of 2.33 and $2.42 \mu_{\mathrm{B}}$, respectively, with the remaining moment on the nonmagnetic $\mathrm{Co}^{3+}$ ions. Therefore, based on the peak positions and magnetic moment, we selected $\left\{\mathrm{U}\left(\mathrm{Co}^{2+}\right), \mathrm{U}\left(\mathrm{Co}^{3+}\right)\right\}=\{4,0\}$ for our calculations.

As shown in Figure 3.7(a), after scaling by photoionization cross-section, [139] the peak positions, valence band width, and relative peak intensities of the calculated DOS (solid black line) are in good agreement with XPS valence band. Site- and orbitalprojected DOS for $\left\{\mathrm{U}\left(\mathrm{Co}^{2+}\right), \mathrm{U}\left(\mathrm{Co}^{3+}\right)\right\}=\{4,0\}$ are shown in Figure 3.7(b). Comparing with the literature, our $\mathrm{U}\left(\mathrm{Co}^{2+}\right)$ value agrees with the value used by Chen et al., $[137,138]$ while our $\mathrm{U}\left(\mathrm{Co}^{3+}\right)$ value agrees with that of Qiao et al. [105] We calculate a band gap of $0.6 \mathrm{eV}$, in agreement with the recent transmission measurements from Qiao et al. [105] The valence band features are dominated by contributions from $\mathrm{Co}^{2+}$ and $\mathrm{Co}^{3+} \mathrm{d}$ states and $\mathrm{O} 2 \mathrm{p}$ states, with strong hybridization between these orbitals. The top of the valence band is mostly composed of $\mathrm{Co}^{3+} \mathrm{d}$ and $\mathrm{O} 2 \mathrm{p}$ states, while the main $\mathrm{O} 2 \mathrm{p}$ states lie deeper in the valence band. The bottom of the conduction band has contributions from $\mathrm{Co}^{2+}$ and 
$\mathrm{Co}^{3+} \mathrm{d}$ states as well as $\mathrm{O} 2 \mathrm{p}$ states. The presence of $\mathrm{Co}^{3+} \mathrm{d}$ states at the top of the valence band is in agreement with the assignments of Kim and Park. [106,136] Since both $\mathrm{Co}^{2+}$ and $\mathrm{Co}^{3+} \mathrm{d}$ states are present at the conduction band minimum, we cannot resolve whether the gap originates from an on-site or cross-site transition.

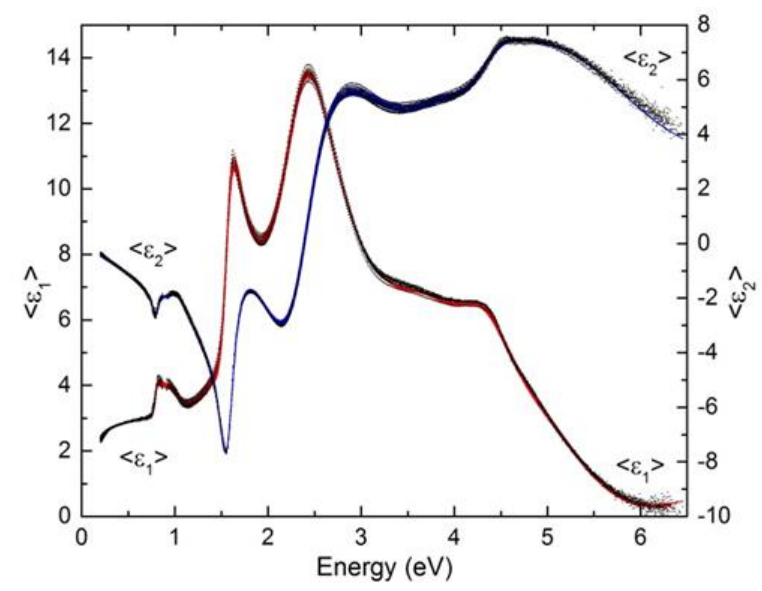

Figure 3.8: Pseudodielectric function $\langle\varepsilon\rangle$ of a $220-\AA$-thick $\mathrm{Co}_{3} \mathrm{O}_{4}$ film on spinel. Symbols: Experimental data. Lines: Fit with the dielectric function shown in Figure 3.9.

The pseudo-dielectric function $\langle\varepsilon\rangle$ for a $220-\AA$-thick $\mathrm{Co}_{3} \mathrm{O}_{4}$ epilayer on spinel is shown in Figure 3.8. It is nearly independent of the angle of incidence. Also, the data from both instruments are well matched in the area of overlap near $0.8 \mathrm{eV}$. As mentioned above, $\langle\varepsilon\rangle$ is not representative of the optical constants for $\mathrm{Co}_{3} \mathrm{O}_{4}$. Instead, it combines features of the layer and the substrate. For example, at low energies (near $0.5 \mathrm{eV}$, below the band gap of the film), $\left\langle\varepsilon_{1}\right\rangle=2.9$, which is determined by the optical constants of the spinel substrate. [129] At intermediate energies (between 0.7 and $3 \mathrm{eV}$ ), the $\mathrm{Co}_{3} \mathrm{O}_{4}$ film causes interference effects and the absorption peaks of $\mathrm{Co}_{3} \mathrm{O}_{4}$ appear as peaks in $\left\langle\varepsilon_{1}\right\rangle$. (In a bulk material, absorption causes peaks in $\left\langle\varepsilon_{2}\right\rangle$.) In the $\mathrm{UV}$, the absorption of the $\mathrm{Co}_{3} \mathrm{O}_{4}$ film is very large and interference effects decrease. Therefore, $\left\langle\varepsilon_{1}\right\rangle$ resembles the dielectric function of the $\mathrm{Co}_{3} \mathrm{O}_{4}$. We also note that interference effects cause $\left\langle\varepsilon_{2}\right\rangle$ to be negative below $2.5 \mathrm{eV}$, where the absorption of $\mathrm{Co}_{3} \mathrm{O}_{4}$ is related to minima of $\left\langle\varepsilon_{2}\right\rangle$. At higher energies, it causes maxima. 
The optical constants of the $\mathrm{Co}_{3} \mathrm{O}_{4}$ epilayer can be determined using well-known procedures, $[130,131]$ since the film thickness and the optical constants of the spinel substrate are known. [129] We write the dielectric function $\varepsilon$ as a sum of Kramers-Kronig consistent oscillators and vary the oscillator parameters until we reach good agreement between the data (symbols) and model (solid lines) in Figure 3.8. The details of the expansion are not important and the fit parameters are not physically meaningful.

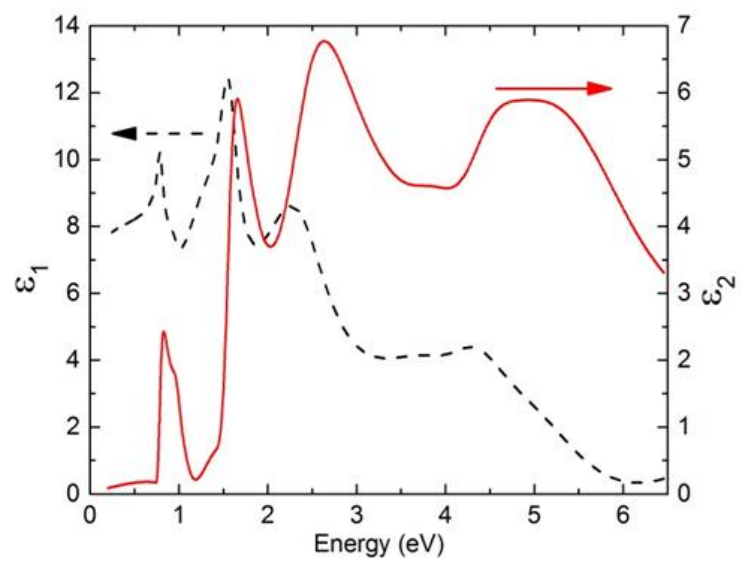

Figure 3.9: Real (dashed) and imaginary (solid) parts of the dielectric function $\varepsilon$ of $\mathrm{Co}_{3} \mathrm{O}_{4}(220-\AA$-thick film grown by MBE) from the near-IR to the near-UV, showing peaks due to interband electronic transitions. The band gap for this $\mathrm{Co}_{3} \mathrm{O}_{4}$ film is $0.75 \mathrm{eV}$.

The resulting dielectric function $\varepsilon$ of $\mathrm{Co}_{3} \mathrm{O}_{4}$ is shown in Figure 3.9. These optical constants are available online. We see that $\mathrm{Co}_{3} \mathrm{O}_{4}$ is an insulator, since $\varepsilon$ vanishes at low energies. It has a direct band gap (onset of strong absorption) of $0.75 \mathrm{eV}$. An indirect band gap or defect absorption is not visible with ellipsometry, since the resulting absorption coefficient is very small. The first absorption peak (maximum in the joint density of states for allowed interband optical transitions) occurs at $0.9 \mathrm{eV}$. Since we merge data sets from two instruments in this spectral region, there is an artifact in the data, which shows a double-peak structure near $0.9 \mathrm{eV}$. (A double peak was also suggested in density-functional calculations [105] but we are unable to confirm this with our current experiments. More precise work, including low-temperature ellipsometry measurements optimized for the near-infrared spectral region are needed.) The absorption then drops and reaches a minimum at $1.2 \mathrm{eV}$. There is a second onset of strong absorption 
near $1.4 \mathrm{eV}$, which has been associated with the direct band gap in previous studies. [106,107] The strong absorption peak at $0.9 \mathrm{eV}$ was not identified in some of the earlier work because of the limited spectral range in some previous experiments. On the other hand, our results are in excellent agreement with those of Ref. [105], where a band gap of $0.74 \mathrm{eV}$ is reported from transmission and photoluminescence measurements. It has been argued [105] that this gap is due to direct dipole-forbidden d-d intraband transitions at tetrahedral-site $\mathrm{Co}^{2+}$ cations. These transitions (forbidden in the presence of perfect spherical symmetry) become allowed due to crystal-field splitting in the cubic spinel structure and due to hybridization of the oxygen $2 \mathrm{p}$ states with the cobalt $3 \mathrm{~d}$ states. See the projected DOS shown in Figure 3.7(b) for comparison. Our calculations also find substantial $\mathrm{Co}^{3+}$ contributions to the valence band maximum and the conduction band minimum. Therefore, we also expect contributions to $d$ to $d$ intraband transitions from the $\mathrm{Co}^{3+}$ sites.

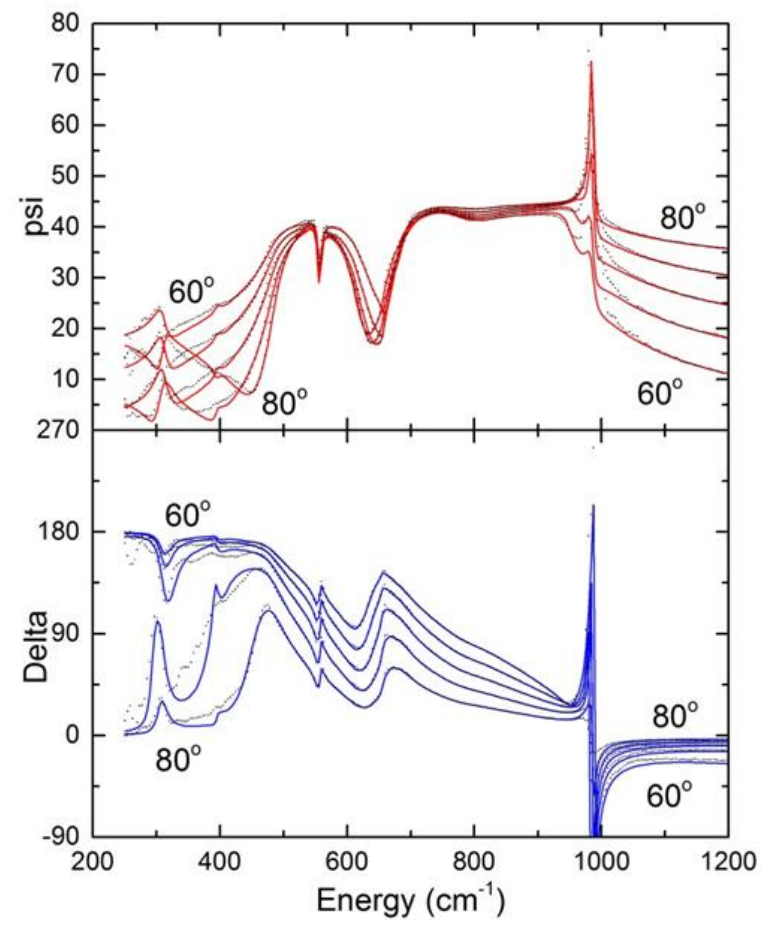

Figure 3.10: Ellipsometric angles for a 220- $\AA$-thick $\mathrm{Co}_{3} \mathrm{O}_{4}$ film on spinel in the mid-infrared spectral region at five angles of incidence from $60^{\circ}$ to $80^{\circ}$. (Symbols: Experimental data. Lines: Fit with a Lorentz oscillator expansion of the $\mathrm{Co}_{3} \mathrm{O}_{4}$ lattice absorption.)

The higher-energy peaks at 1.65 and $2.6 \mathrm{eV}$ were previously identified by Kim and Park. [106] Our work shows an additional broad absorption peak at $5 \mathrm{eV}$, comparable to Ref. [105]. At even higher energies, $\varepsilon_{2}$ drops significantly. 
The ellipsometric angles in the region of the lattice absorption by infrared active phonons in the $\mathrm{Co}_{3} \mathrm{O}_{4}$ epilayer and the spinel $\mathrm{MgAl}_{2} \mathrm{O}_{4}$ substrate are shown in Figure 3.10. The data (symbols) can be described with a model (lines) consisting of a spinel substrate and a layer, in which the lattice absorption is written as a sum of Lorentzian lineshapes. [129]

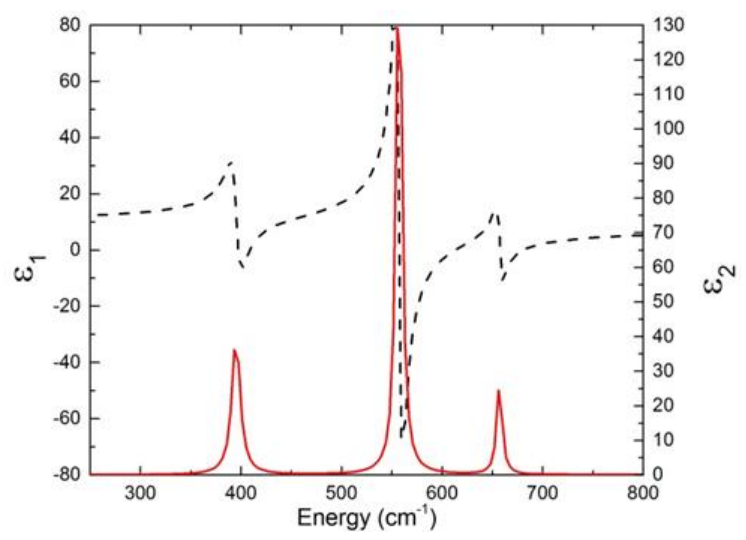

Figure 3.11: Real (dashed) and imaginary (solid) parts of the dielectric function of $\mathrm{Co}_{3} \mathrm{O}_{4}$ in the mid-IR, showing lattice absorption by three infrared-active phonons.

The spectra are very similar to those of bulk spinel, see Fig. 10 in Ref. [129], with four notable exceptions due to the presence of the $\mathrm{Co}_{3} \mathrm{O}_{4}$ epilayer: (1) There is a dip in the first reststrahlen band $\psi$ at $555 \mathrm{~cm}^{-1}$ because of a strong $\mathrm{Co}_{3} \mathrm{O}_{4}$ phonon. (2) At $982 \mathrm{~cm}^{-1}, \psi$ becomes larger than $45^{\circ}$, where the dielectric function of the substrate is close to 1 at an LO peak of the substrate. This is an interference effect. For a bulk material, $\psi$ is always below $45^{\circ}$. (3) A $\mathrm{Co}_{3} \mathrm{O}_{4} \mathrm{TO}$ phonon at $655 \mathrm{~cm}^{-1}$ causes a tiny modification in the rise of the second reststrahlen band. (4) The fit is not good below $400 \mathrm{~cm}^{-1}$, which was attributed to cation disorder in the earlier work on spinel. [129] Nevertheless, we are able to find a small feature in $\psi$ at $395 \mathrm{~cm}^{-1}$, where a $\mathrm{Co}_{3} \mathrm{O}_{4}$ phonon is expected. In summary, we find $\mathrm{Co}_{3} \mathrm{O}_{4}$ phonon peaks at 395,557 , and $656 \mathrm{~cm}^{-1}$, in agreement with FTIR reflectance and electron energy loss measurements. $[107,140,141]$ In the spinel structure, we expect four infraredactive TO phonons. [129] For $\mathrm{Co}_{3} \mathrm{O}_{4}$, the literature also reports an infrared-active $\mathrm{T}_{1 \mathrm{u}}$ phonon at $214 \mathrm{~cm}^{-1}$, which is below the spectral range of our infrared ellipsometer and therefore was not observed by us. 
In Figure 3.11, we show an estimate for the mid-IR dielectric function of $\mathrm{Co}_{3} \mathrm{O}_{4}$, which was obtained from the fit to the data in Figure 3.10. The peak at $395 \mathrm{~cm}^{-1}$ is influenced by cation disorder in the substrate. The peak at $656 \mathrm{~cm}^{-1}$ is close to a TO mode in the substrate. Therefore, the amplitudes, positions, and broadenings of these peaks are not very accurate. On the other hand, the $\mathrm{Co}_{3} \mathrm{O}_{4} \mathrm{TO}$ peak at $656 \mathrm{~cm}^{-1}$ is not influenced by any substrate features and can be determined with good accuracy. The Lorentzian broadening of this TO peak is about $6 \mathrm{~cm}^{-1}$, limited by the spectral resolution $\left(8 \mathrm{~cm}^{-1}\right)$ of our experiment.

We do not observe any signs of a metallic response, which would lead to discrepancies between our lattice-dynamical model and the data at low frequencies. Therefore, we are unable to investigate a two-dimensional electron gas, which might be present at the interface between $\mathrm{Co}_{3} \mathrm{O}_{4}$ and $\mathrm{MgAl}_{2} \mathrm{O}_{4}$. Also, we do not find any signs of free carriers due to doping in the $\mathrm{Co}_{3} \mathrm{O}_{4}$ layer. (Other $\mathrm{Co}_{3} \mathrm{O}_{4}$ layers were reported to be $p$ type. [105])

Finally, magnetization of a $240-\AA$-thick $\mathrm{Co}_{3} \mathrm{O}_{4}$ (110) film was measured as a function of temperature under zero-field-cooled conditions at a constant magnetic field of 1T oriented in-plane (Figure 3.12). Field-cooled measurements were in good agreement with the zero-field-cooled values. The data includes a signal that can be attributed to a background diamagnetic \& paramagnetic contribution from substrate, [142] backing, and sample mount as compared to the small signal from the film. Despite the strong background contribution, a local maximum in the magnetization is clearly visible at $49 \mathrm{~K}$. This value is higher than the Néel temperature $\mathrm{T}_{\mathrm{N}} \approx 40 \mathrm{~K}$ for a $\mathrm{Co}_{3} \mathrm{O}_{4}$ crystal. [110] The $\mathrm{T}_{\mathrm{N}}$ enhancement appears due to the stress created by lattice-parameter mismatch between $\mathrm{Co}_{3} \mathrm{O}_{4}$ and $\mathrm{MgAl}_{2} \mathrm{O}_{4}$. Since the lattice parameter of $\mathrm{Co}_{3} \mathrm{O}_{4}$ is slightly smaller than that of $\mathrm{MgAl}_{2} \mathrm{O}_{4}$, the mismatch places $\mathrm{Co}_{3} \mathrm{O}_{4}$ under a tension stress. It has been widely observed 
that $\mathrm{T}_{\mathrm{N}}$ in antiferromagnetic oxides including $\mathrm{Co}_{3} \mathrm{O}_{4}$ increases under high pressure. $[143,144]$ Why a tension stress increases $T_{N}$ in a G-tpye antiferromagnet deserves further study. Alternatively, a much enhanced $\mathrm{T}_{\mathrm{N}}$ could be related to the change in the oxygen stoichiometry.

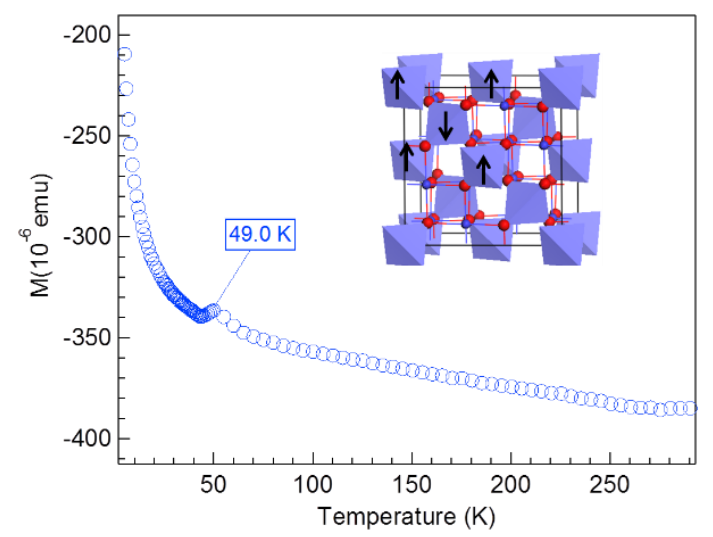

Figure 3.12: SQUID magnetometer: Magnetization of a 240- $\AA$-thick $\mathrm{Co}_{3} \mathrm{O}_{4}$ (110) film was measured as a function of temperature under zero-field-cooled conditions (blue circles) with constant in-plane magnetic field of 1 T. Fieldcooled data show similar characteristics. The onset of antiferromagnetism is labeled at $49 \mathrm{~K}$. Inset image shows G-type antiferromagnetic ordering in the spinel structure. The overall negative magnetization is from the diamagnetic contribution from the substrate.

\subsection{Conclusion}

In conclusion, we have grown $\mathrm{Co}_{3} \mathrm{O}_{4}$ films on $\mathrm{MgAl}_{2} \mathrm{O}_{4}$ (110) using $\mathrm{MBE}$. We investigated surface roughness and smoothing during growth with RHEED and AFM, and further affirm the high quality and sharp interface of the as-grown film and by XRD and TEM. Ellipsometry measurements find a small gap of $0.75 \mathrm{eV}$, in good agreement with DFT calculations based on the shape of the XPS valence band. A valence band offset of $3.2 \mathrm{eV}$ is measured for the heterostructure. Finally, we find that the films are antiferromagnetic with a transition temperature of $49 \mathrm{~K}$, higher than the typical bulk 
value. These results motivate future study of this material, including DFT/TEM study to determine atomic positions at the interface \& surface, low-temperature ellipsometry and optical Hall-effect measurements, and growth on different substrates to study changes in the magnetic properties, band offsets, and catalytic activity. 


\title{
Chapter 4: Quasi-two-dimensional electron gas at the epitaxial alumina/ $/ \mathrm{SrTiO}_{3}$ interface
}

\author{
Contents of this Chapter were published in J. Appl. Phys. 117, 95303
}

(2015) [145].t.

In this chapter, we report on the highly conducting layer formed at the crystalline $\gamma$-alumina/ $/ \mathrm{SrTiO}_{3}$ (STO) interface which is attributed to oxygen vacancies. We describe the structure of thin $\gamma$-alumina layers deposited by molecular beam epitaxy on STO (001) at growth temperatures in the range of 400 to $800^{\circ} \mathrm{C}$, as determined by reflection-highenergy electron diffraction, $\mathrm{x}$-ray diffraction, and high-resolution electron microscopy. In situ x-ray photoelectron spectroscopy was used to confirm the oxygen-deficient layer. Electrical characterization indicates sheet carrier densities $\sim 10^{13} \mathrm{~cm}^{-2}$ at room temperature for the sample deposited at $700^{\circ} \mathrm{C}$. A maximum electron Hall mobility of $560 \mathrm{~cm}^{2} \mathrm{~V}^{-1} \mathrm{~s}^{-1}$ at $15 \mathrm{~K}$ and room temperature mobility of $5 \mathrm{~cm}^{2} \mathrm{~V}^{-1} \mathrm{~s}^{-1}$ are measured and annealing in Oxygen is found to reduce the carrier density and turn a conductive sample into an insulator.

\subsection{INTRODUCTION}

$\mathrm{SrTiO}_{3}(\mathrm{STO})$ has received much attention because of its large dielectric constant and its role in the integration of other complex oxides on semiconductors, with applications including catalysis, tunable devices, and ferroelectric functionality. [79,80,101,146-150] A highly interesting application for STO involves the formation of a high mobility two-dimensional electron gas (2DEG) at the oxide/oxide

\footnotetext{
$\$$ Contributions: KJK designed and performed the work related to the sample growth, RHEED, XRR, and XPS and analyzed the data. AAD, ABP, TQN, and JGE contributed to the conception and analysis of data. JJ-S performed and analyzed the XRD results. SL, DJS, and MRM performed TEM experiments. NG and XPAG performed and analyzed electrical measurements. All authors contributed to interpretation of the data and to discussions.
} 
interface. $[11,13,22-24,44,45,116,151-156]$ Among the mechanisms for 2DEG formation, one approach involves tailoring an interface between STO and oxides with a large negative enthalpy of formation, such as aluminum-based oxides. $[13,44,45,116,154-156]$ Under such conditions, it is energetically favorable for oxygen atoms near the interface to diffuse out of the STO during growth, stabilizing a confined conducting layer. [13,44,45,116,154-158] Since this metallic layer results from the formation of oxygen vacancies near the interface, it characteristically vanishes with oxygen atmospheric anneal. [44,45,116,155,156]

Previous studies of crystalline $\gamma-\mathrm{Al}_{2} \mathrm{O}_{3}$ grown by pulsed laser deposition (PLD) have shown that the confinement of the conducting layer depends on growth parameters, particularly substrate temperature. [116,155] Furthermore, even amorphous oxide heterostructures grown on STO by PLD and atomic layer deposition (ALD) exhibit interfacial conductivity. $[44,45,156]$ Considering that the diffusivity of oxygen in alumina varies widely depending on crystal structure, $[159,160]$ the properties of the vacancy-controlled STO 2DEG need to be quantified with respect to thin film deposition parameters. The molecular beam epitaxy (MBE) method employed herein facilitates the fabrication of well-ordered oxide heterostructures with precise layer-by-layer atomic control, without complicating factors such as the plume dynamics of PLD [44,116,155] or precursor reactivity of ALD. $[45,156]$ In this chapter, we describe the structure of MBE-grown crystalline $\gamma$-alumina on STO (001) as determined by reflection-high-energy electron diffraction (RHEED), x-ray diffraction (XRD), and high-resolution electron microscopy (HREM). The conducting STO layer exhibits a strong dependence on the growth temperature, as revealed by x-ray photoelectron spectroscopy (XPS), and electrical measurements. 


\subsection{EXPERIMENTAL DETAILS}

$\mathrm{SrTiO}_{3}(001)$ substrates with dimensions $5 \mathrm{~mm} \times 5 \mathrm{~mm} \times 0.5 \mathrm{~mm}$ (commercially available with $\mathrm{TiO}_{2}$-termination by $\mathrm{HF}$ etching from Crystec) were degreased in acetone, isopropanol, deionized water, and UV ozone. The samples were then introduced into a customized DCA $600 \mathrm{MBE}$ system with a base pressure of $6 \times 10^{-10}$ Torr. More details of the experimental system can be found elsewhere. [87,161] All substrates were outgassed in the MBE chamber at $700^{\circ}$ for 30 min under ultra-high vacuum (UHV). The substrate temperature was measured by a thermocouple (calibrated by pyrometer measurement of a silicon substrate) in close proximity to the substrate heater.

The substrate temperature during alumina deposition was varied between 400 to $800^{\circ} \mathrm{C}$. Al metal flux evaporated from an effusion cell was calibrated to a $\gamma$-alumina deposition rate of $1.8 \AA / \mathrm{min}$ as measured by a quartz crystal microbalance. Molecular oxygen was introduced at a background pressure of $1 \times 10^{-6}$ Torr. The samples were monitored during growth by in situ RHEED. After film deposition, the main shutter was closed, and the samples were cooled down in the presence of oxygen $\left(1 \times 10^{-6}\right.$ Torr).

\subsection{RESULTS AND DISCUSSION}

STO assumes the cubic $P m 3 m$ perovskite structure above $110 \mathrm{~K}$, [162] while the cubic $\gamma$-phase of $\mathrm{Al}_{2} \mathrm{O}_{3}$ is based on the $F d \overline{3} m$ spinel structure with $\mathrm{Al}$ vacancies to satisfy stoichiometry. $[163,164]$ The measured distances between the diffraction lines in the STO and alumina RHEED patterns (Figure 4.1(a)) indicate the lattice relationship represented in Figure 4.1(b), with an alumina lattice parameter of $7.9 \AA$. [163,164] The oxygen sublattice of the spinel matches closely to that of the perovskite substrate, with a lattice mismatch of $-1 \%$. X-ray reflectivity analyses was performed using a Panalytical X'PERT Pro diffractometer $(\mathrm{Cu} \mathrm{K} \alpha 1$ source, $\lambda=1.5406 \AA)$ operating at $40 \mathrm{kV}$ and $30 \mathrm{~mA}$. An x-ray reflectivity pattern of an estimated 10-nm alumina/STO sample is also shown in 
Figure 4.1(b). The strong, regular oscillations indicate excellent uniformity and smoothness of the film. Additional x-ray diffraction was carried out at the National Synchrotron Light Source beamline X20A (Figure 4.1(c)). The low-intensity shoulder to the left of the main STO (002) peak is attributed to alumina. Due to weak scattering from aluminum combined with close proximity to strong STO substrate peaks, strain analysis of the film is not possible; however, in-plane scans (not shown) indicate cubic symmetry. To further characterize the samples, we examined the alumina/STO interfaces using cross-sectional transmission electron microscopy.

(a)

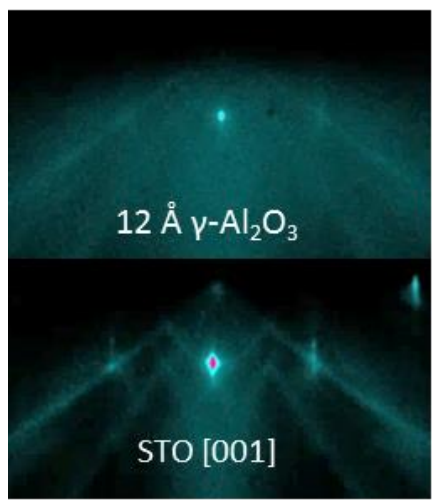

(b)

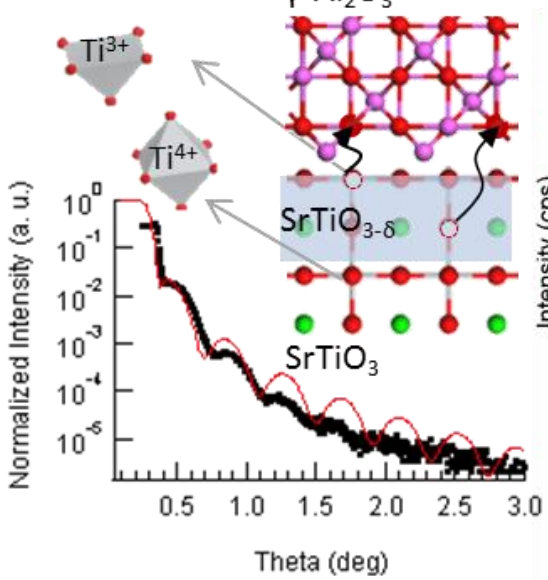

(c)

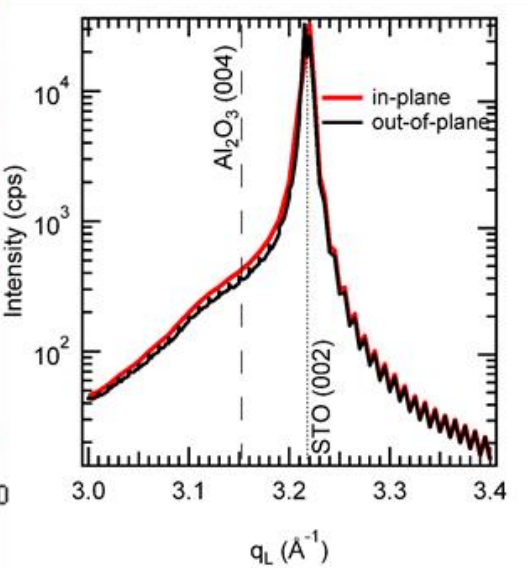

Figure 4.1: (a) In situ RHEED of the crystalline STO and alumina surfaces. (b) X-ray reflectivity of structure and interface with inset epitaxial relationship of $\gamma$ alumina on STO (001). (c) Bragg-Brentano scans along the (001) direction.

Figure 4.2 shows a representative micrograph recorded with a JEOL JEM4000EX transmission electron microscope operated at $400 \mathrm{keV}$. This image along the [100] direction clearly reveals an abrupt interface between the highly crystalline epitaxial alumina and the STO substrate. The structural model (inset) shows that the STO substrate 
is terminated with a $\mathrm{TiO}_{2}$ layer, while the spinel side of the interface commences with aluminum in the 4-fold coordinated tetrahedral site.

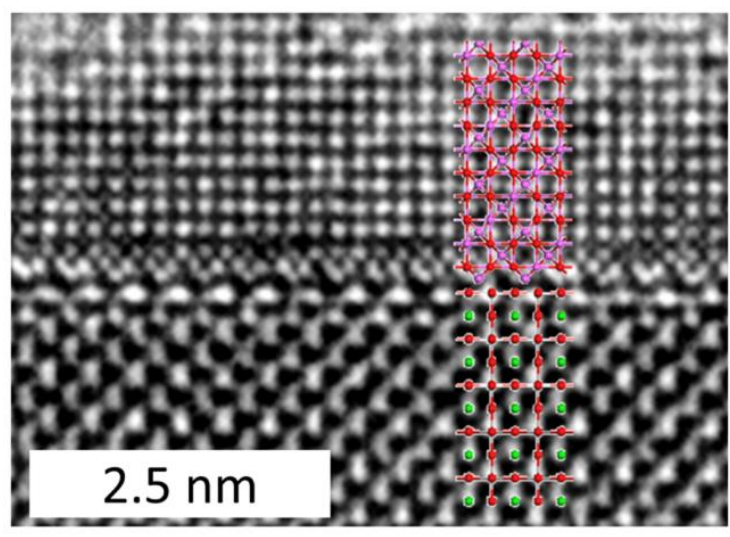

$2.5 \mathrm{~nm}$

Figure 4.2: High-resolution electron micrograph in cross-section geometry confirming abrupt interface between highly crystalline epitaxial alumina on STO substrate.

XPS measurements were performed in situ using a VG Scienta R3000 with monochromatic Al $\mathrm{K} \alpha$ radiation $(\mathrm{h} v=1486.6 \mathrm{eV})$. As illustrated in Figure 4.3(a), we determine the valence-band offset between the STO substrate and a thick (7-nm) alumina film by aligning the spectra such that: (1) the Sr $3 d$ core level (CL) in the heterostructure (3-nm alumina/STO) matches that of the pure STO substrate, and (2) the Al $2 p$ CL in the thick alumina film matches that of the heterostructure, $V B O_{C L}=\left(E_{S r 3 d}-E_{V B M}\right)_{S T O}-\left(E_{A l 2 p}-E_{V B M}\right)_{A_{2} O_{3}}-\left(E_{S r 3 d}-E_{A l 2 p}\right)_{A_{2} O_{3} / S T O_{4}}$.

A valence-band offset of $+0.93 \mathrm{eV}$ was calculated and also verified by simulating a thin-film valence band (Figure 4.3(b)), as described elsewhere. [87] As the two methods returned the same value, no band bending was detected. [165,166] Furthermore, the binding energy of the $\mathrm{Al} 2 p$ core level $(76 \mathrm{eV})$ and shape of the alumina valence band indicate no sign of any Al suboxide. [164,167] 

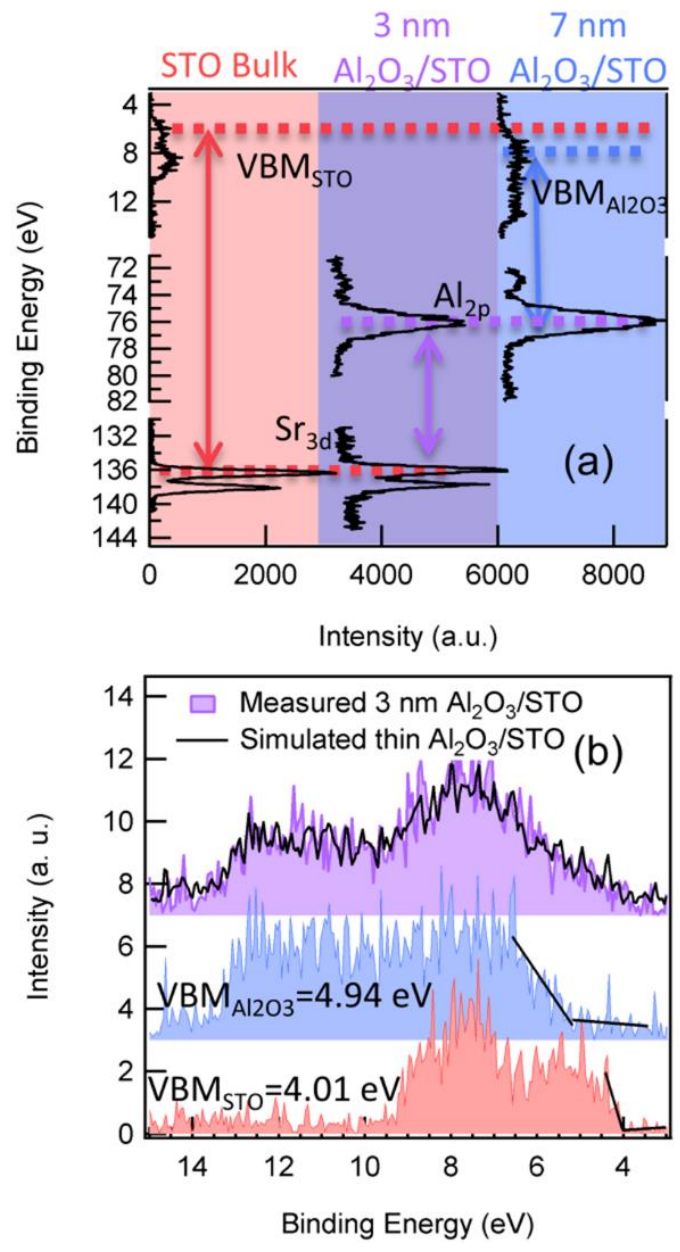

Figure 4.3: Core level (a) and valence band (b) spectroscopy for the following: STO substrate (red), $3 \mathrm{~nm}$ alumina/STO (purple), and $7 \mathrm{~nm}$ alumina/STO (blue).

The $\mathrm{Ti} 2 p$ core level provides information on the oxygen vacancy concentration on the STO side of the interface. As illustrated in Figure 4.4(a), peak decomposition of the Ti $2 p$ core level in STO allows a comparison of fully oxidized and reduced Ti. While the bulk STO substrate (purple solid line) shows only two spin-split $\mathrm{Ti}^{4+}$ (fully oxidized) peaks, the spectrum after MBE growth of a thin alumina film (raw data: light blue solid line, background: bright blue solid line, fit: black solid line) shows peaks at

lower binding energies corresponding to $\mathrm{Ti}$ in a reduced environment $\left(\mathrm{Ti}^{3+}\right.$ : dark blue dashed filled line, $\mathrm{Ti}^{2+}$ : navy dashed filled line) in addition to the main peak from fully oxidized $\mathrm{Ti}\left(\mathrm{Ti}^{4+}\right.$ : light blue dashed filled line). The percentage of remaining fully oxidized $\mathrm{Ti}^{4+}$ was then calculated by comparing the relative peak areas, $C_{4+}=\frac{I\left(T i^{4+}\right)}{I\left(T i^{4+}\right)+I\left(T i^{3+}\right)+I\left(T i^{2+}\right)}$.

This analysis of the XPS $\mathrm{Ti} 2 p$ spectra was repeated for samples of different alumina thicknesses $(0-7 \mathrm{~nm})$ grown at 4 different substrate temperatures $\left(400-800^{\circ} \mathrm{C}\right.$ in 
$100^{\circ} \mathrm{C}$ increments), as summarized in Figure 4.4(b). The resulting depth profile indicates the presence of an interfacial oxygen-deficient STO layer.

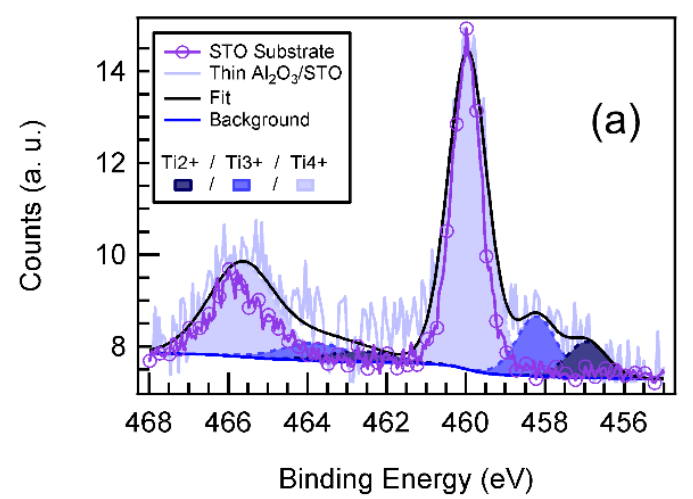

Figure 4.4: (a) Peak decomposition of the Ti $2 p$ core level in STO allows comparison of fully oxidized and reduced Ti. (b) Analysis of reduced titanium based on XPS Ti $2 p$ spectra for different samples of different thickness and growth temperature.

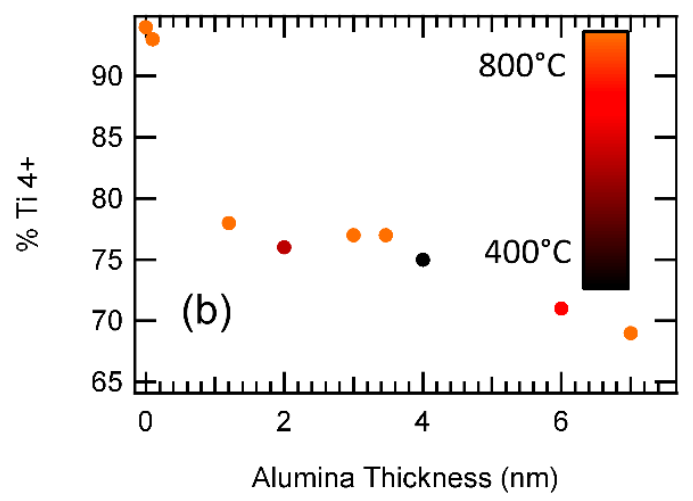

In order to electrically contact the alumina/STO interface, four indium contacts were placed on scribed corners of each sample in a Van der Pauw geometry. Measurements took place in a Quantum Design physical property measurement system (PPMS) capable of applying a $\pm 9 \mathrm{~T}$ magnetic field and 1.9-350 $\mathrm{K}$ temperature range. Two Stanford SR830 lock-in amplifiers and one SR570 current preamplifier were used to perform 4-wire electrical transport measurement using less than $1 \mu \mathrm{A}$ current at a frequency of 7 or $13 \mathrm{~Hz}$. Conductivity measurements as a function of temperature using a 4-wire lock-in measurement reveal metallic behavior for the interface between these two insulators (Figure 4.5(a)). Comparison between two samples (one deposited at a substrate temperature of $400^{\circ} \mathrm{C}$ and the other at $700^{\circ} \mathrm{C}$ ) indicates a higher sheet resistance for lower growth temperature. 

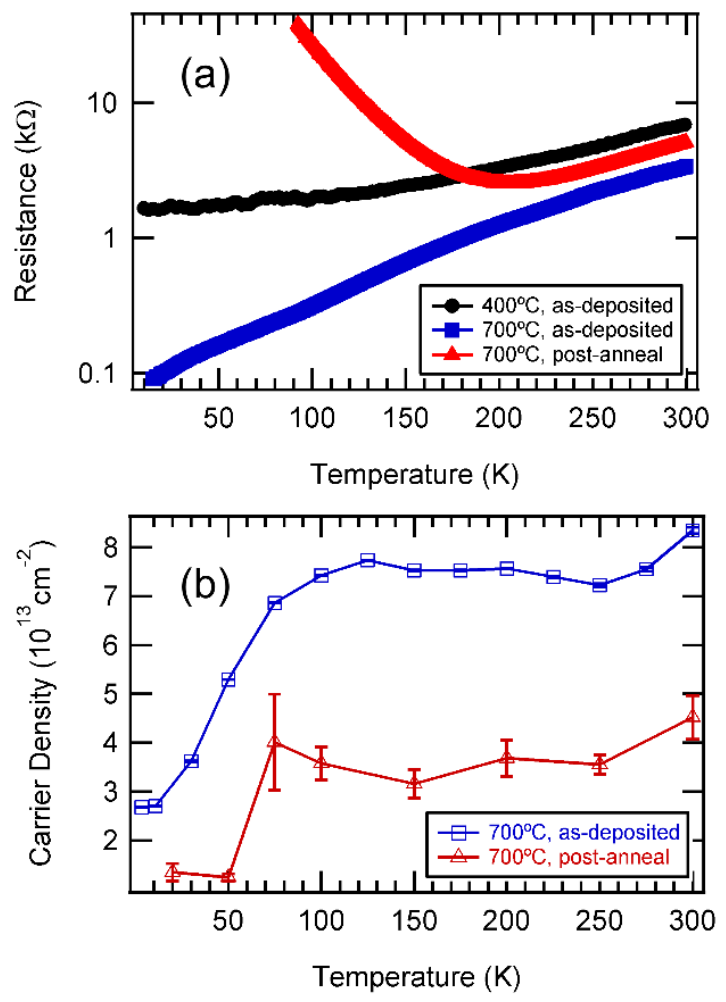

Figure 4.5: (a) Sheet resistance of MBEgrown alumina/STO samples grown with deposition temperatures of $700^{\circ} \mathrm{C}(6 \mathrm{~nm}$ $\left.\mathrm{Al}_{2} \mathrm{O}_{3}\right)$ and $400^{\circ} \mathrm{C}\left(4 \mathrm{~nm} \mathrm{Al}_{2} \mathrm{O}_{3}\right)$. Annealing the $700^{\circ} \mathrm{C}\left(6 \mathrm{~nm} \mathrm{Al}_{2} \mathrm{O}_{3}\right)$ sample in 1 Torr of $\mathrm{O}_{2}$ at $400^{\circ} \mathrm{C}$ for 5 minutes turned the sample's $R(T)$ into an insulating behavior below $200 \mathrm{~K}$. (b). Temperature dependent Hall carrier density of $700^{\circ} \mathrm{C}(6$ $\mathrm{nm}$ ) sample before and after the annealing in $\mathrm{O}_{2}$ ( 1 Torr, $400^{\circ} \mathrm{C}$ for 5 minutes). Hall coefficient was measured using a $\pm 5 \mathrm{~T}$ magnetic field.

Sheet carrier densities on the order of $10^{13}-10^{14} \mathrm{~cm}^{-2}$ are measured in the sample deposited at $700^{\circ} \mathrm{C}$ (Figure $4.5(\mathrm{~b})$, blue squares), with a rapid drop in its temperature dependence below $110 \mathrm{~K}$,

coinciding with the cubic to tetragonal phase transition of the STO substrate. [162] A maximum electron Hall mobility of $3100 \mathrm{~cm}^{2} \mathrm{~V}^{-1} \mathrm{~s}^{-1}$ at $3.2 \mathrm{~K}$ and room temperature mobility of $22 \mathrm{~cm}^{2} \mathrm{~V}^{-1} \mathrm{~s}^{-1}$ are measured for the heterostructure. For the sample deposited at $400^{\circ} \mathrm{C}$, we were unable to obtain reliable Hall measurement. However, if one assumes similar carrier density as the sample grown at $700^{\circ} \mathrm{C}$, then the carrier mobility is roughly two times lower at room $\mathrm{T}$ and ten times lower at $10 \mathrm{~K}$. To elucidate the oxygen vacancy effect on the carrier density and transport behavior, we annealed the $700^{\circ} \mathrm{C}$ deposited ( $6 \mathrm{~nm} \mathrm{Al}_{2} \mathrm{O}_{3}$ ) sample in 1 Torr of $\mathrm{O}_{2}$ at $400^{\circ} \mathrm{C}$ for 5 minutes. After annealing, the sample showed nearly halved carrier density (Figure 4.5(b), red triangles) and an insulating behavior at low temperature (Figure 4.5(a), red triangles). This attests to the oxygen vacancy origin of carriers at the conductive interface. 


\subsection{Conclusion}

In conclusion, we demonstrate the growth of crystalline $\gamma$-alumina on STO using molecular beam epitaxy. X-ray photoelectron spectroscopy of Ti $2 p$ core level indicates an oxygen-deficient layer at the interface. Electrical measurements reveal that the interfacial layer exhibits an increased sheet resistance for decreased growth temperature. Future investigations will study the interface structure in more detail, and its influence on 2DEG mobility and the dimensionality of the conducting layer as determined by Shubnikov-de Hass oscillations. These properties can then be compared with results using other film deposition methods.

The work at UT and ASU was supported by the Air Force Office of Scientific Research (AFOSR) (FA9550-12-10494). The work at CWRU was supported by AFOSR grant FA9550-12-1-0441. Use of the National Synchrotron Light Source, Brookhaven National Laboratory, was supported by the U.S. Department of Energy, Office of Science, Office of Basic Energy Sciences, under Contract No. DE-AC02-98CH10886. Use of facilities in the John M. Cowley Center for High Resolution Electron Microscopy at ASU is also acknowledged. 


\section{Chapter 5: Positive linear magnetoresistance in the two-dimensional electron gas at the $\mathrm{EuO} / \mathrm{SrTiO}_{3}$ interface}

The development of novel nano-oxide spintronic devices would greatly benefit from interfacing with emergent phenomena at oxide interfaces. In this chapter, we integrate highly spin-split ferromagnetic semiconductor $\mathrm{EuO}$ onto $\mathrm{SrTiO}_{3}(001)$. The $\mathrm{EuO}$ growth by molecular beam epitaxy results in oxygen out-diffusion from $\mathrm{SrTiO}_{3}$ leaving behind a highly conductive layer on the $\mathrm{SrTiO}_{3}$ side of the interface through generation of oxygen vacancies. Below the Curie temperature of $70 \mathrm{~K}$ of EuO a spinpolarized two-dimensional electron gas at the $\mathrm{EuO} / \mathrm{SrTiO}_{3}$ interface displays positive linear magnetoresistance. This system offers an as-yet-unexplored route to pursue proximity-induced phenomena in the oxide two-dimensional electron gas.

\subsection{INTRODUCTION}

The high mobility two-dimensional electron gas (2DEG) at the oxide/oxide interface is currently under intense investigation [11]. In particular, different types of magnetism have been observed in the oxide 2DEG [39] providing a richness of physical phenomena ripe for the continuing development of novel oxide devices [31]. Thin films of perovskite oxides exhibit superconductonductivity [168] and colossal magnetoresistance [169], magnetism [170], $\quad$ ferroelectricity [171] and multiferroicity [172], piezoelectricity [173], and thermoelectricity [174]. On the other

hand, a nearly ideal Heisenberg ferromagnet, rock salt EuO boasts a saturation magnetic moment of $7 \mu_{\mathrm{B}}$ (with corresponding $0.6 \mathrm{eV}$ spin-splitting of the unoccupied $5 d$ band [175]). Thus EuO can be used for spin filtering, [176] and is considered a strong candidate for spintronic applications [176]. There also have been proposals to combine 
EuO with Al thin films [177], graphene layers [178] and $\mathrm{MoTe}_{2}$ [179] layers, to induce ferromagnetism in these systems by proximity effect.

Therefore, it is of great interest to look for novel physical phenomena at the interface of ferromagnetic semiconductor $\mathrm{EuO}$ and other complex oxides. First-principles calculations predict a fully spin-polarized 2DEG at the $\mathrm{LaAlO}_{3} / \mathrm{EuO}$ interface due to electrostatic doping from the polar oxide $[180,181]$. However, from a thermodynamic perspective, creation of the heterostructure suggested by Lee et al. [181] is somewhat problematic. EuO is not stable under ambient conditions [182], much less the oxygen-rich high-temperature conditions necessary for deposition of crystalline $\mathrm{LaAlO}_{3}$ [183]. Among the various mechanisms for the oxide 2DEG formation, one viable approach involves tailoring an interface between $\mathrm{SrTiO}_{3}$ (STO) and oxides with large negative enthalpy of formation to stabilize a confined conducting layer of $\mathrm{SrTiO}_{3-\delta}$. $[116,145] \mathrm{As}$ $\mathrm{EuO}$ is one such oxide, [184] the approach offers an as-yet-unexplored route to investigate proximity-induced phenomena in the oxide $2 \mathrm{DEG}$.

Here we demonstrate positive linear magnetoresistance of the 2DEG at the interface of epitaxial $\mathrm{EuO} / \mathrm{STO}$, for $\mathrm{EuO}$ film thicknesses in the range $\sim 5-10 \mathrm{~nm}$ grown by molecular beam epitaxy (MBE). Growth is achieved by depositing Eu metal onto STO (001) without oxygen in UHV. The x-ray diffraction (XRD) and scanning transmission electron microscopy (STEM) show that epitaxy on $\mathrm{TiO}_{2}$-terminated STO plane results in the rock salt $\mathrm{EuO}\left(\mathrm{Eu} 2^{+}\right)$. The $\mathrm{EuO}$ thin films are ferromagnetic below the Curie

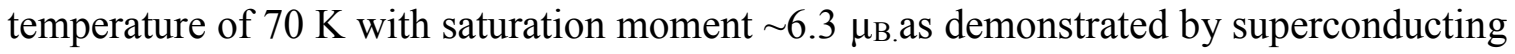
quantum interference device (SQUID) magnetometry and low-temperature transport measurements. These EuO/STO heterostructures display temperature-dependent linear positive magnetoresistance below the Curie temperature. X-ray photoemission spectroscopy (XPS) shows the valence band offset of $2 \mathrm{eV}$ and closely aligned 52 
conduction bands. The density functional theory (DFT) analysis based on the XRD/STEM-derived structural model provides a consistent picture of the band alignment, magnetic state of $\mathrm{EuO}$ and electronic structure of the oxygen deficient conductive layer formed in STO. Using soft x-ray angle-resolved photoemission spectroscopy (SX-ARPES), we elucidate the $d_{x y} \mathrm{t}_{2 \mathrm{~g}}$ character of the low dimensional electron system. The carriers reside at the STO side of the EuO/STO interface, which conclusively demonstrates symmetry breaking due to carrier confinement and thus the existence of the 2DEG. Combining these results with first-principles calculations we uncover the role of the spin-polarized oxygen vacancy as the origin of the linear positive magnetoresistance stemming from the ferromagnetism of $\mathrm{Eu}^{2+}$ magnetic moments in proximity to the confined oxygen-deficient conductive layer.

\subsection{EXPERIMENTAL DETAILS}

\subsubsection{Film deposition}

$\mathrm{SrTiO}_{3}(001)$ substrates with dimensions $5 \mathrm{~mm} \times 5 \mathrm{~mm} \times 0.5 \mathrm{~mm}$ (commercially available with $\mathrm{TiO}_{2}$-termination by $\mathrm{HF}$ etching from Crystec) were degreased in acetone, isopropanol, deionized water, and UV ozone. The samples were then introduced into a customized DCA $600 \mathrm{MBE}$ system with a base pressure of $6 \times 10^{-10}$ Torr. More details of the experimental system can be found elsewhere. [185] All substrates were outgassed in the MBE chamber at $700^{\circ}$ for 10 min under ultra-high vacuum (UHV). The substrate temperature was measured by a thermocouple (calibrated by pyrometer measurement of a silicon substrate) in proximity to the substrate heater.

The substrate temperature during $\mathrm{EuO}$ deposition was fixed at $200^{\circ} \mathrm{C}$. Eu metal flux evaporated from an effusion cell was calibrated to a metal deposition rate of $\sim 0.36$ $\mathrm{nm} / \mathrm{min}$ as measured by a quartz crystal microbalance. Molecular oxygen was introduced 
at a partial pressure varied between $1 \times 10^{-10}$ to $1 \times 10^{-8}$ Torr. The samples were monitored during growth in situ by RHEED. After film deposition, the films were capped with $\sim 1.4$ $\mathrm{nm}$ aluminum metal to form $\sim 2$-nm alumina upon exposure to ambient conditions for $e x$ situ electrical and magnetic characterization.

\subsubsection{Sample Characterization}

XPS measurements were performed in situ using a VG Scienta R3000 electron energy analyzer with monochromatic $\mathrm{Al} \mathrm{K} \alpha$ radiation $(\mathrm{h} v=1486.6 \mathrm{eV})$. Superconducting quantum interference device magnetic moment measurements of a 7-nm-thick EuO (001) film were recorded as a function of temperature under field-cooled conditions at a constant magnetic field of .01 T oriented in-plane.

To electrically contact the capped EuO/STO interface, four indium contacts were placed on corners of each sample in a van der Pauw geometry. Measurements were performed with a Physical Property Measurement System (PPMS) capable of applying a $\pm 9 \mathrm{~T}$ magnetic field. The magnetic properties were measured with a Superconducting Quantum Interference Device (SQUID) from Quantum Design.

\subsubsection{Density functional theory}

First-principles calculations based on density functional theory (DFT) were performed using generalized gradient approximation [186] (GGA) for the projector augmented wave pseudopotentials [187], as implemented in the Vienna Ab-Initio

Simulation Package code [188]. For Sr, Ti, Eu and O, $4 s^{2} 4 p^{6} 5 s^{2}, 3 s^{2} 3 p^{6} 4 s^{2} 3 d^{2}, 5 s^{2} 5 p^{6} 4 f$ ${ }^{7} 6 s^{2}$ and $2 s^{2} 2 p^{4}$ are included, respectively. The plane-wave cutoff energy was $600 \mathrm{eV}$. To correct the on-site Coulomb interaction and consider the correlation effect in $\mathrm{SrTiO}_{\mathrm{v}}$, we adopted Dudarev's rotationally invariant approach [189] adding a Hubbard U term $(\mathrm{GGA}+\mathrm{U})$. Typical values $\mathrm{U}_{\mathrm{f}}=5.0 \mathrm{eV}$ and $\mathrm{U}_{\mathrm{d}}=5 \mathrm{eV}, \mathrm{J}_{\mathrm{d}}=0.64 \mathrm{eV}$ were used for $\mathrm{Eu}$ 
localized $4 f$ orbitals and $\mathrm{Ti} 3 d$ orbitals, respectively. We employed symmetric $(\mathrm{EuO})_{3}(\mathrm{STO})_{6}(\mathrm{EuO})_{3}$ supercell geometry with vacuum region thicker than $1 \mathrm{~nm}$ to prevent interaction between adjacent slabs. The interface was $\mathrm{TiO}_{2}$-terminated and $\mathrm{Eu}$ atoms were on top of hollow positions in $\mathrm{TiO}_{2}$ plane, as continuation of $\mathrm{Sr}$ atom. Lattice parameter asтo $=0.395 \mathrm{~nm}$ was used and $\mathrm{EuO}$ layers were rotated by $45^{\circ}$ to match the lattice constant ( $8 \%$ tensile strain on EuO layers). For creation of a single vacancy, an $\mathrm{O}$ atom was removed at the sub-interface $\mathrm{SrO}$ layer in a $2 \times 2$ slab. All atom positions were fully relaxed until residual forces were less than $0.2 \mathrm{eV} \mathrm{nm}^{-1}$. The Brillouin zone was sampled with $4 \times 4 \times 1$ Monkhorst-Pack $k$-point grids [190].

\subsubsection{Soft-X-ray ARPES experiments}

The experiments have been carried out at the SX-ARPES endstation [191] of the ADRESS beamline [192] at the Swiss Light Source (Paul Scherrer Institute, Switzerland). Circularly polarized X-rays were incident on the sample at a grazing angle of $20^{\circ}$ to increase photoelectron yield from the buried $\mathrm{EuO} / \mathrm{SrTiO}_{3}$ interface. The sample was cooled down to $12 \mathrm{~K}$ to quench the thermal effects reducing the coherent k-resolved spectral component at high photoexcitation energies [193]. The combined (beamline and analyzer) energy resolution was $\sim 100 \mathrm{meV}$. The SX-ARPES resonant measurements at the Ti $L$-edge were complemented by X-ray adsorption spectroscopy (XAS) measurements in total electron yield.

With an intense photon flux of about $2 \times 10^{13} \mathrm{ph} / \mathrm{sec}$ delivered by the ADRESS beamline into a spot of $\sim 30 \times 74 \mu \mathrm{m}^{2}$ on the sample, the SX-ARPES spectra significantly depended on the X-ray irradiation dose due to photogenerated oxygen vacancies. This can be seen in time evolution of the SX-ARPES images presented in Appendix A, Figure A.9 through A.11. The irradiation apparently increases the spectral intensity and band 
filling. To avoid any significant distortion of the pristine $\mathrm{EuO} / \mathrm{SrTiO}_{3}$ spectral response, our SX-ARPES spectra were recorder under continuous translation of the sample under the beam.

\subsection{RESULTS AND DISCUSSION}

\subsubsection{Sample Preparation and Characterization}

Since $\mathrm{EuO}$ is highly sensitive to oxygen pressure and tends to form $\mathrm{Eu}_{2} \mathrm{O}_{3}$, special care must be taken to ensure proper stoichiometry. In general, EuO epitaxy must be carefully controlled with regards to temperature, deposition rate, and oxygen pressure to preserve the $\mathrm{Eu}^{2+}$ oxidation state; metallic $\mathrm{Eu}^{0}$ has a low sticking coefficient [194], while

over-oxidized $\mathrm{Eu}^{3+}$ is paramagnetic [195]. Here, we build upon the previous multi-metal study [184] of oxygen scavenging from $\mathrm{SrTiO}_{3}$ and demonstrate that it is possible to crystallize stoichiometric EuO by depositing Eu metal onto $\mathrm{SrTiO}_{3}(001)$ under ultra-high vacuum, where oxygen is provided only by the substrate. Details of the growth window were investigated by in-situ x-ray photoelectron spectroscopy as summarized in Appendix A, Figure A.1. For ex situ characterization, a capping layer of 2-nm $\mathrm{AlO}_{\mathrm{x}}$ was deposited directly after growth. For observations by scanning transmission electron microscopy (STEM), a 10-nm Ti capping layer was deposited to protect the surface from oxidation during STEM sample cross-sectioning for viewing along $\mathrm{SrTiO}_{3}[100] / \mathrm{EuO}$ [110] projection.

The EuO films crystallize in the rock salt structure (Space group Fm3m [196]) and the primary unit cell axis is rotated by $45^{\circ}$ with respect to the unit cell axis of substrate surface to minimize lattice mismatch (22\% down to $\sim 7 \%)$. The films are fully relaxed, as shown schematically in Figure 5.1(a) and in the reciprocal space map in Figure 5.1(b). Additional x-ray diffraction results are provided in Appendix A, Figure 
A.2. Lattice parameters extracted from the in-plane and out-of-plane scans are 0.513 and $0.515 \mathrm{~nm}$, respectively.

(a)
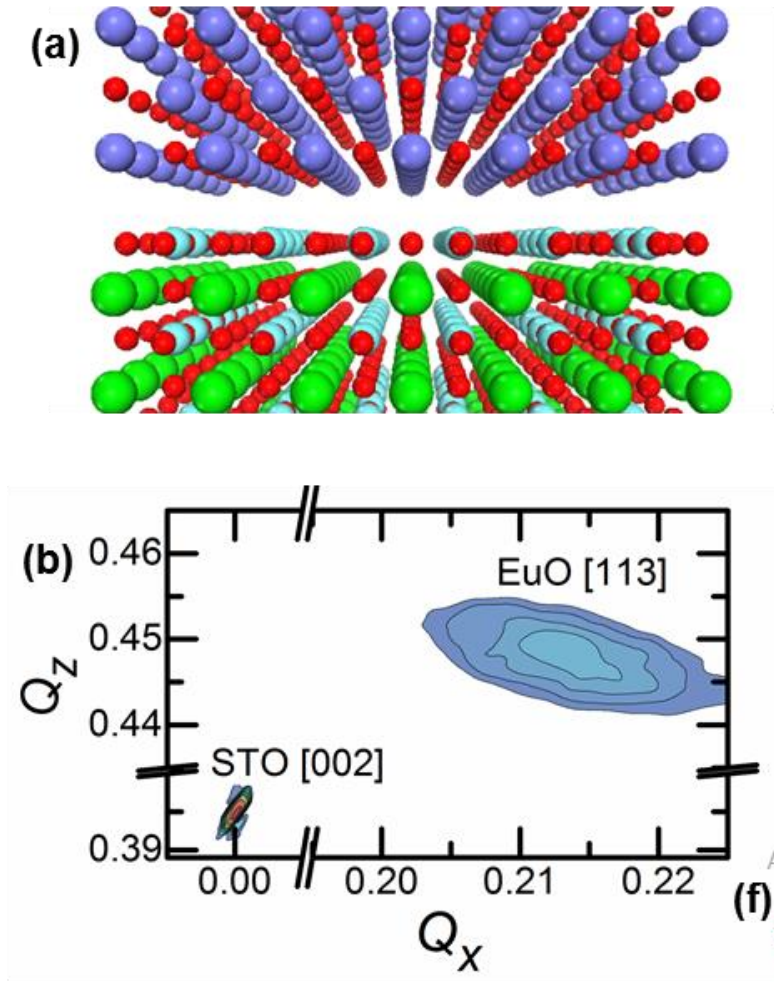

(c)

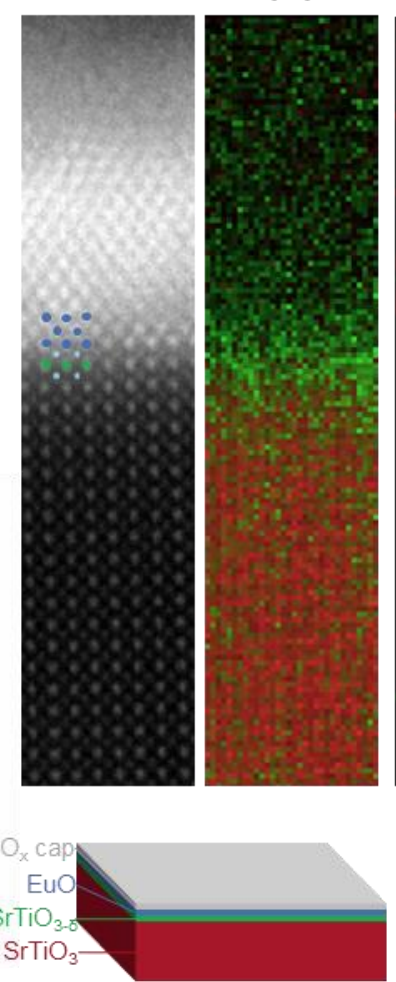

(e)

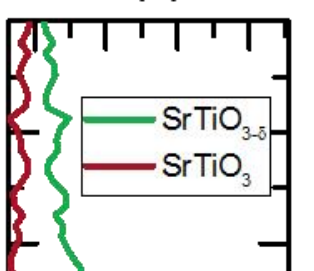

6

4

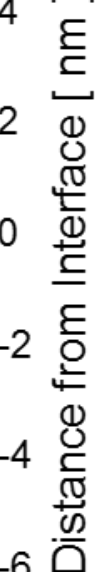

$-6$

Figure 5.1: Epitaxy of $\mathrm{EuO}$ on $\mathrm{SrTiO}_{3}$ (001). (a) Atomic model of the rocksalt / perovskite heterointerface. (b) Reciprocal space map of the STO (002) and $\mathrm{EuO}(113)$ peaks for $7 \mathrm{~nm} \mathrm{EuO} \mathrm{on} \mathrm{STO}$. The EuO rocksalt unit cell is rotated $45^{\circ}$ with respect to the surface unit cell of the perovskite. (c) Highangle annular-dark-field scanning transmission electron microscopy [100]projection image of the EuO/STO interface. (d) Corresponding false color map shows a distribution map from the Ti L-edge fit $\left(\mathrm{SrTiO}_{3}\right.$, red; $\mathrm{SrTiO}_{3-\delta}$, green). (e) Ti-L coefficient as a function of position shows a sharp peak at the interface. (f) Overall schematic, including the bulk STO substrate (red), layer of STO with oxygen vacancies (green), EuO film (blue), and capping layer (gray). Not to scale.

As shown in the high-angle annular-dark-field image of the $\mathrm{EuO} / \mathrm{SrTiO}_{3}$ interface in Figure 5.1(c), the films are epitaxial with defects in the first few layers. From the Ti-L 
energy-loss near-edge fine-structure edge fitting [197], false color maps in Figure 5.1(d) show the distribution of oxygen vacancies at the interface. This result is in good qualitative agreement with $\mathrm{x}$-ray photoelectron spectroscopy results reported for Eu metal on $\mathrm{SrTiO}_{3}$ by Posadas et al [184]. Complementary Eu-N edge data confirming $\mathrm{Eu}^{2+}$ oxidation state are given in Appendix A, Figure A.3. The proximity of EuO to the confined $\mathrm{SrTiO}_{3-\delta}$ conducting layer is shown in the heterostructure cross-section in Figure $5.1(\mathrm{e})$

\subsubsection{Electrical Characterization}

The EuO film exhibits a paramagnetic to ferromagnetic transition with decreasing temperature as seen in Figure 5.2(c), which shows the field-cooled magnetization of a 7$\mathrm{nm} \mathrm{EuO} \mathrm{film} \mathrm{as} \mathrm{a} \mathrm{function} \mathrm{of} \mathrm{temperature.} \mathrm{Curie-Weiss} \mathrm{fit} \mathrm{to} \mathrm{this} \mathrm{data} \mathrm{give} \mathrm{a} \mathrm{Curie}$ temperature of $T_{\mathrm{C}} \sim 70 \mathrm{~K}$ and effective magnetization of $\sim 6.3 \mu_{\mathrm{B}}$. From the magnetization loops measured at $10 \mathrm{~K}$ with magnetic fields applied in the plane of the film [see inset to Figure 5.2 (c)] we extract a coercive field $\sim 0.02 \mathrm{~T}$ and remnant magnetization $\sim 4.3 \mu_{\mathrm{B}}$. These are essentially bulk values for EuO.

Measurements of the sheet resistance $R_{\mathrm{S}}$ over a temperature range from 2-300 K reveal metallic behavior (Appendix A, Figure A.4(a)) for 7-nm EuO/STO. Hall measurements indicate high sheet carrier densities on the order of $10^{16} \mathrm{~cm}^{-2}$ (Appendix A, Figure A.4(b)). Figure 5.2 (a) shows four-probe magnetoresistance $R_{\mathrm{S}}(B)$ measurements for a 7-nm $\mathrm{EuO}$ film in a perpendicular magnetic field. $R_{\mathrm{S}}$ increases linearly with the magnetic field at $20 \mathrm{~K}$ and quadratically at $100 \mathrm{~K}$. Solid lines indicate fits to the data of the form,

$R_{S}(B)=R_{S}(0) \times\left[c_{1}|B|+\left(c_{2} B\right)^{2}\right]$ 

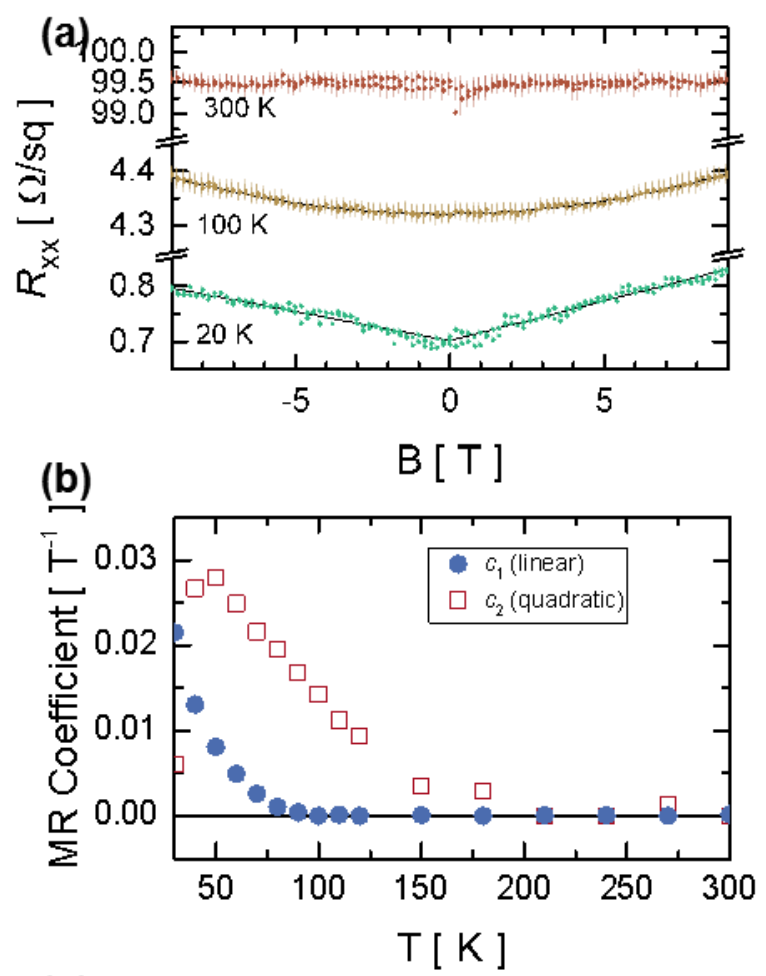

(c)

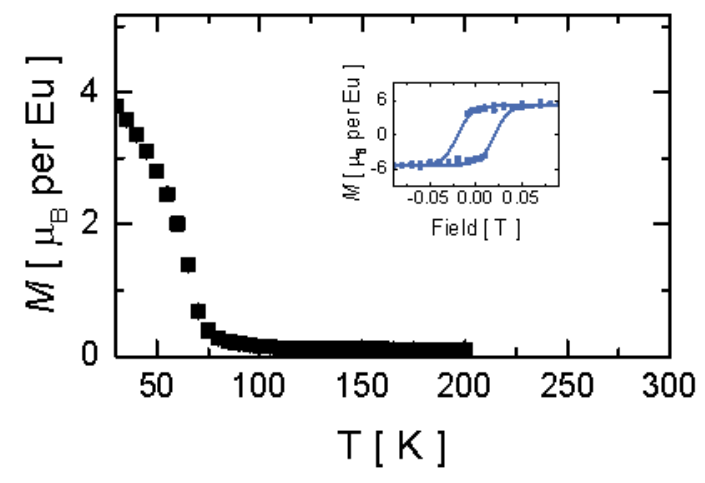

Figure 5.2: (a) Magnetoresistance (MR) data measured in a perpendicular magnetic field at $20 \mathrm{~K}, 100 \mathrm{~K}$, and 300 $\mathrm{K}$. Solid lines indicate fits to the data. The MR increases linearly with the magnetic field at $20 \mathrm{~K}$, quadratically at $100 \mathrm{~K}$, and is field-independent at room temperature. (b) Linear $c_{1}$ and quadratic $c_{2}$ MR fit coefficients for the same film as a function of temperature. (c) Fieldcooled magnetization $M$ of a similar 7$\mathrm{nm} \mathrm{EuO} \mathrm{film} \mathrm{as} \mathrm{a} \mathrm{function} \mathrm{of}$ temperature at constant in-plane magnetic field of $0.01 \mathrm{~T}$. Inset: corresponding magnetization loop measured at $10 \mathrm{~K}$.

where $c_{1}$ and $c_{2}$ are the linear and quadratic fit coefficients, respectively, shown in Figure 5.2(b) as a function of temperature. The quadratic magnetoresistance component is present below $\sim 150 \mathrm{~K}$, while the linear component emerges below $\sim 80 \mathrm{~K}$. The magnetoresistance (MR), defined as, $M R=\frac{R_{S}(B)-R_{S}(0)}{R_{S}(0)}$,

decreases rapidly as the measurement temperature increases, and is essentially zero at room temperature as shown in Figure 5.2(a).

The quadratic MR component mentioned above can be attributed to the ordinary magnetoresistance, as in normal metals, stemming from the Lorentz force. On the other hand, a much more interesting linear MR needs special consideration. Since its 
emergence coincides with the Curie temperature $(\sim 70 \mathrm{~K})$, we ascribe the origin of the positive linear MR to a Zeeman split of the 2DEG electronic structure [198] induced by magnetic order of oxygen vacancies in top STO layer, as DFT modelling illustrates below.

\subsubsection{First-principles Calculations}

For these $\mathrm{EuO} / \mathrm{SrTiO}_{3-\delta} / \mathrm{SrTiO}_{3}(001)$ heterostructures, the band alignment is crucial in determining the spatial extent of the conducting $\mathrm{SrTiO}_{3-\delta}$ layer and therefore the magnitude of the wave function overlap which is the origin of the exchange proximity interaction [199]. Band offsets were measured by x-ray photoelectron spectroscopy, as described in Appendix A and Figure A.6. The theoretical density of states (DOS) and valence band offset are in good agreement with the experimental XPS data, also shown in Figure 5.3 (the details of the first-principles calculations of the $\mathrm{EuO} / \mathrm{SrTiO}_{3}$ interface are given in above). Figure 5.3 shows the simulation cell with one oxygen vacancy in the sub-interface $\mathrm{SrO}$ layer and the corresponding DOS projected onto atomic planes across the heterostructure. The interface structure in the calculations is kept consistent with STEM images recorded in the [110] projection (Appendix A, Figure A.5).

A sharp, spin-up Eu $4 \mathrm{f}$ state forms the valence band edge and is fully spinpolarized, with a magnetic moment of $7 \mu_{B}$. This is in good agreement with studies of ferromagnetism in bulk $\mathrm{EuO}$ [200]. A localized impurity state emerges $0.4 \mathrm{eV}$ below Fermi level. This state, residing on two Ti ions adjacent to a vacancy, has an $e_{g}$ orbital character mixed with $p_{z}$ due to lifting of the local cubic symmetry induced by a vacancy [201]. Importantly, the in-gap state is singly-occupied and polarized with its spin aligned with the Eu ion above the interface. The impurity state decays quickly into both $\mathrm{EuO}$ and $\mathrm{SrTiO}_{3}$, with the evanescent states present up to 2 layers away from the vacancy 
plane. The decay length is $0.19 \mathrm{~nm}$ and $0.18 \mathrm{~nm}$ in EuO and STO respectively, consistent with the complex band structure [202].

(a)
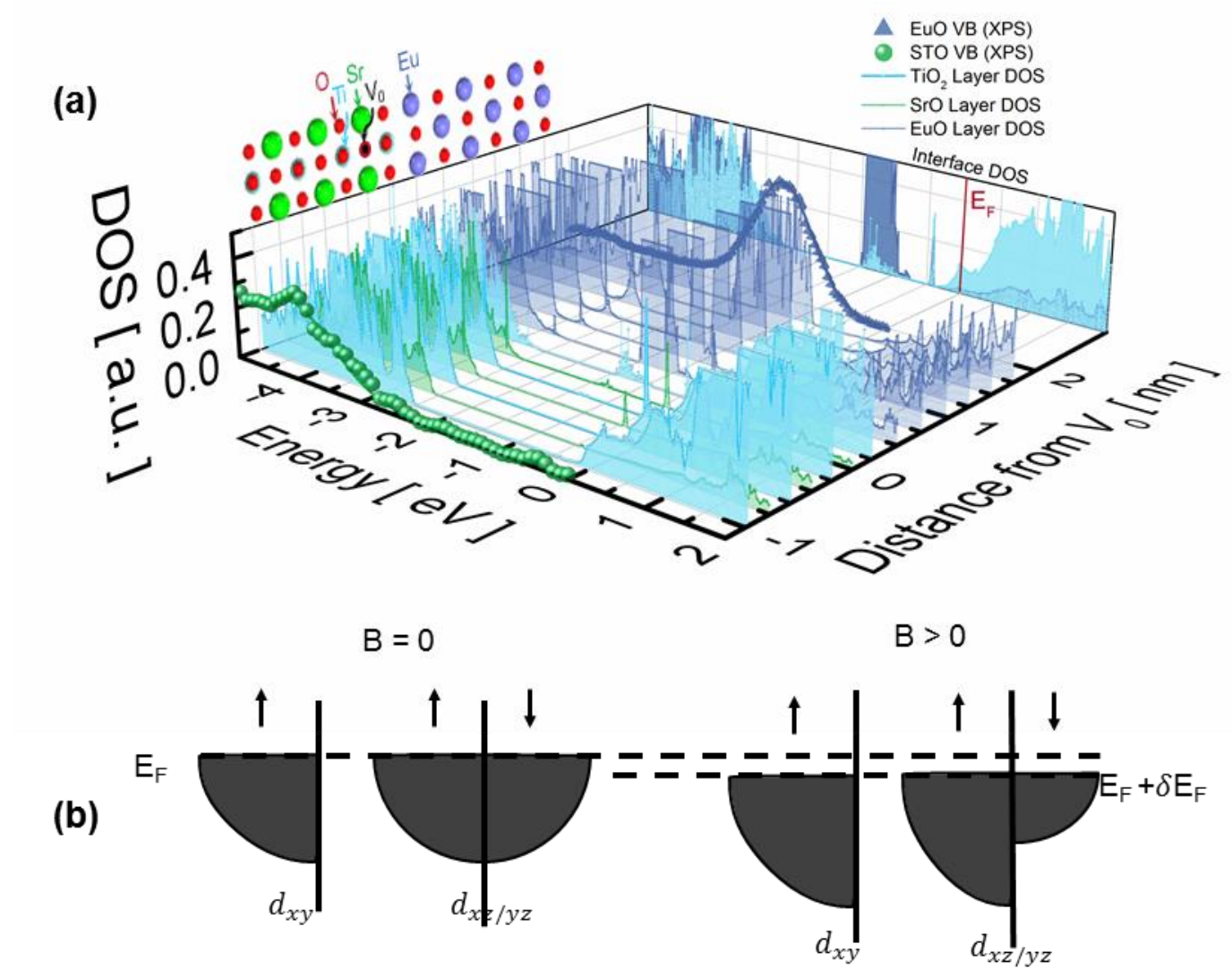

Figure 5.3: (a) Density of states (DOS) projected onto atomic planes across the EuO/STO simulation cell, with an oxygen vacancy $\left(\mathrm{V}_{0}\right)$ at the STO surface. The oxygen vacancy state can be seen at $-0.4 \mathrm{eV}$. This state decays within $\sim 0.2$ $\mathrm{nm}$ from the interface. The theoretical valence band (VB) shapes and valence band offset are in good agreement with experimental data, also shown. (b) Schematic illustration of Zeeman shift.

Inspecting carriers in the conduction band of $\mathrm{SrTiO}_{3}$, we note that all itinerant electrons are located on the $\mathrm{SrTiO}_{3}$ side and the delocalized Ti $t_{2 g}$ states are occupied by the second electron of the vacancy. Recent studies by Hou et al. [203] and Lin et al. [157], suggested that the vacancy-induced localized state can trap at most one 
electron, while the second electron occupies the conduction band when correlation effects are taken into account. From the orbital-projected DOS (Appendix A, Figure A.7), we find that the occupied states at the interface are purely $d_{x y}$ orbitals, but mainly $d_{x z} / d_{y z}$ orbitals away from the interface. The split-off $d_{x y}$ band has also been found at the $\mathrm{LaAlO}_{3} / \mathrm{SrTiO}_{3}$ interface and attributed to the orbital reconstruction due to symmetry lowering [22]. It is worth noting that the occupied $d_{x y}$ state at the interface is also spinsplit by $\sim 0.3 \mathrm{eV}$ and thus the interfacial carriers are fully spin-polarized in the same way as the vacancy-induced in-gap state and the $\mathrm{Eu} 4 \mathrm{f}$ state.

To explain the positive linear MR we consider the Zeeman shift of spin-split bands. Below the critical temperature $T_{\mathrm{c}}, \mathrm{EuO}$ becomes ferromagnetic and as suggested by calculation (Appendix A, Figure A.7), carriers at the interface $\left(d_{x y}\right)$ will be spinpolarized while carriers in deeper layers $\left(d_{x z / y z}\right)$ remain nonmagnetic. With an external field $\mathrm{H}$, the spin-polarized $d_{x y}$ band and the initially-nonmagnetic up band shift downward while the initially-nonmagnetic down band shifts upward, as shown in Figure 5.3(b). Following the work of Onose et al. [198] as detailed in Appendix A, the change of the resistivity suggests a positive linear MR with magnetic field. Another possible mechanism for the positive linear MR is quantum electron-electron interference [204]. However, following the work of Lee et al. [204] and Gerber et al. [205], the calculated quantum correction is several orders of magnitude smaller compared with our measurement. Our results suggest that below $70 \mathrm{~K}$, ferromagnetism in the EuO layer causes the alignment of spin-polarized oxygen vacancies in STO. This causes the linear positive MR of the $d_{x y}$ carriers at the interface stemming from proximity induced defect magnetism. 


\subsubsection{Photoemission}

To visualize band structure of the $\mathrm{EuO} / \mathrm{SrTiO}_{3}$ interface resolved in electron momentum k, we used soft-x-ray angle-resolved photoemission spectroscopy (SXARPES). Spectral response of the buried interface states was boosted using resonant photoexcitation at the Ti $2 p$ absorption edge. In Figure 5.4, we present the experimental (a) X-ray absorption spectra and (b) resonant (angle integrated) photoemission intensity across the Ti $2 p$ edge. The latter embeds the Ti $t_{2 \mathrm{~g}}$ derived 2DEG signal at $E_{\mathrm{F}}, \mathrm{Eu} 4 f$ around $E_{\mathrm{B}} \sim-2.5 \mathrm{eV}$ and the $\mathrm{O} 2 p$ derived valence band states of $\mathrm{EuO}$ and $\mathrm{SrTiO}_{3}$ below. Figure 5.4(c) shows the photoemission intensity variations in the corresponding $E_{\mathrm{B}}$ regions. The $2 \mathrm{DEG}$ and valence band response resonates near the Ti absorption peaks. This confirms, respectively, the Ti $3 d$ origin of the 2DEG and hybridization of the O $2 p$ states with $\mathrm{Ti}$, similarly to the paradigm $\mathrm{LaAlO}_{3} / \mathrm{SrTiO}_{3}$ interface $[206,207]$. On the other hand, the $\mathrm{Eu} 4 f$ response shows no correlation with the Ti $2 p$ absorption that indicates vanishing hybridization between the $\mathrm{Eu} 4 f$ and Ti $3 d$ states. Furthermore, similar resonant

data at the Eu $3 d$ absorption edge (Appendix A, Figure A.8) indicates no sign of any significant admixture of Eu $4 f$ states in the 2DEG. This indicates that the 2DEG in the $\mathrm{EuO} / \mathrm{SrTiO}_{3}$ heterostructure resides on the $\mathrm{SrTiO}_{3}$ side of the interface, in good agreement with density functional calculations. 

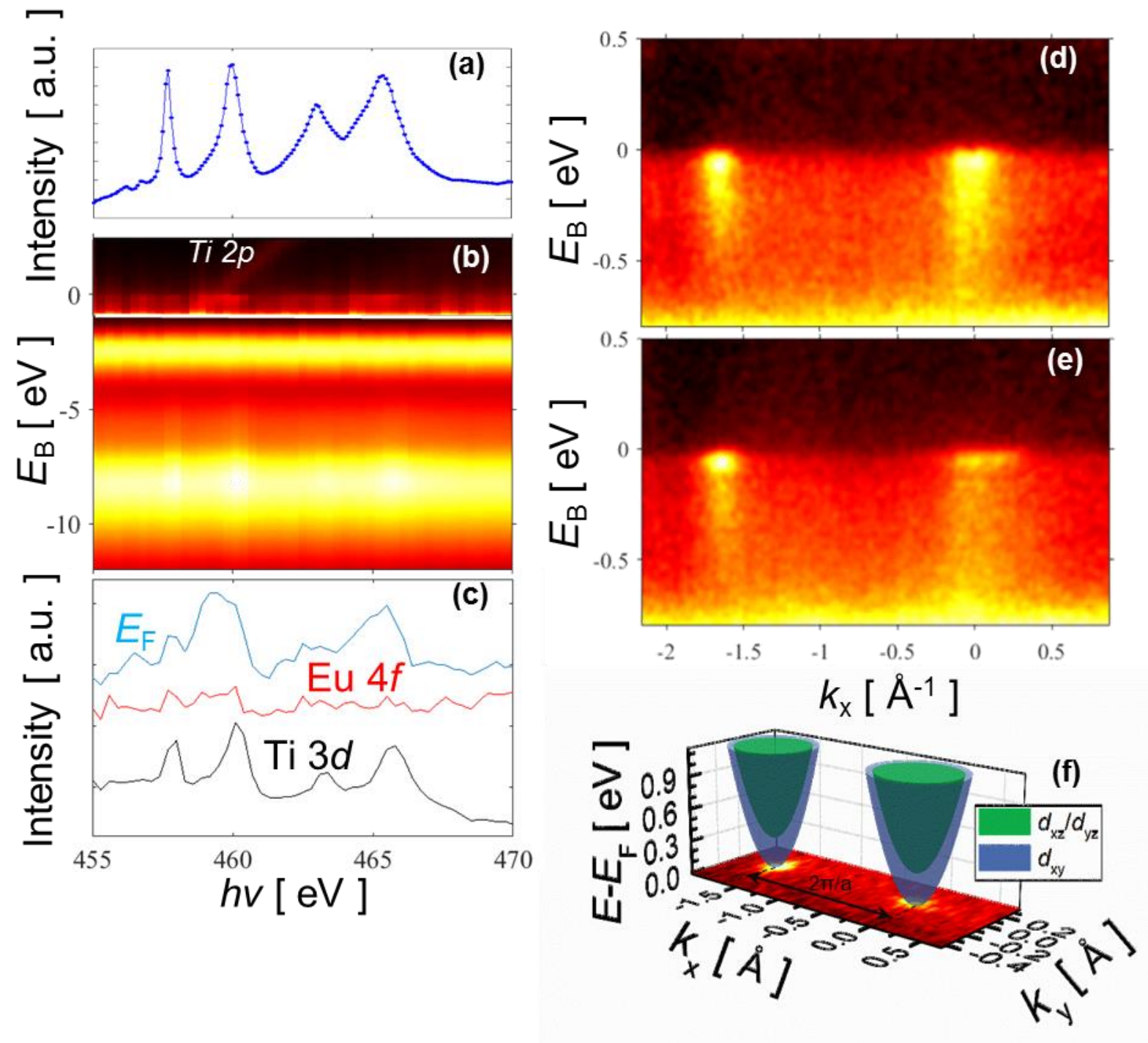

Figure 5.4: Resonant soft-X-ray ARPES of 2-nm EuO/STO heterointerface through the Ti $L$-edge. (a) XAS spectrum. (b) Resonant photoemission from the valence band as a function of excitation energy. Intensity the near- $E_{\mathrm{F}}$ region is scaled up by $\sim 30$. (c) Resonant intensity for constant $E_{\mathrm{B}}$ in the valence band, $\mathrm{Eu}$ level and 2DEG. The valence band and 2DEG signals follow the Ti $L$ edge XAS spectrum that confirms their Ti-derived character. (d,e) SXARPES images at $h v=460.3$ and $466 \mathrm{eV}$ enhancing the $d_{\mathrm{xy}}$ and $d_{\mathrm{yz}}$-derived states, respectively. The intensity waterfalls are reveal polaronic nature of the interface charge carriers. (f) Fermi surface of the interface states measured at $h v=466 \mathrm{eV}$. 
Photoelectron images visualizing electron dispersions $E(\mathbf{k})$ in the 2DES are shown in Figure 5.4(d) for $h v=460.3 \mathrm{eV}$ emphasizing the Ti $d_{\mathrm{xy}}$ states localized near the interface, and Figure 5.4(e) for $466 \mathrm{eV}$ emphasizing the Ti $d_{\mathrm{yz}} / d_{\mathrm{xz}}$ ones more extended into the $\mathrm{SrTiO}_{3}$ bulk [207]. This is consistent with our DFT calculations in the previous section. The EuO/SrTiO 3 interface shows much smaller band filling compared to the $\mathrm{LaAlO}_{3} / \mathrm{SrTiO}_{3}$ case [207] that is manifested by small Fermi vector $k_{\mathrm{F}}$ of the heavy $d_{\mathrm{yz}}$ band (e). The waterfalls going from the band dispersions down in $E_{\mathrm{B}}$ is a hallmark of all $\mathrm{SrTiO}_{3}$-based systems that signal polaronic nature of the charge carriers with their characteristic peak-dip-hump spectral response involving electron coupling to the LO3 phonon [207,208]. Furthermore, significantly smaller intensity of the $\mathrm{EuO} / \mathrm{SrTiO}_{3}$ interface bands compared to $\mathrm{LaAlO}_{3} / \mathrm{SrTiO}_{3}$ may reveal larger fraction of the nonconducting interfacial phase [209,210]

Finally, Figure 5.4(f) shows the Fermi surface formed by the interface electrons. It was measured at $h v=466 \mathrm{eV}$ to emphasize the external contours formed by the ellipsoidal $\mathrm{Ti} d_{\mathrm{yz}} / d_{\mathrm{xz}}$ sheets. As expected from the experimental $E(\mathbf{k})$ dispersions, the Fermi surface is nevertheless dominated by the circular $d_{x y}$ derived electron pocket with only small filling of the $d_{\mathrm{yz}} / d_{\mathrm{xz}}$ sheets compared to the $\mathrm{LaAlO}_{3} / \mathrm{SrTiO}_{3}$ case [207]. Therefore, the overall electron density has in our case stronger interface localization compared to $\mathrm{LaAlO}_{3} / \mathrm{SrTiO}_{3}$ interface.

\subsection{CONCLUSION}

In summary, we have discovered the linear positive $\mathrm{MR}$ in the $\mathrm{EuO} / \mathrm{SrTiO}_{3}$ ${ }_{\delta} / \mathrm{SrTiO}_{3}$ heterostructure grown by depositing of $\mathrm{Eu}$ metal onto $\mathrm{SrTiO}_{3}$ (001). Such deposition enables crystallization of stoichiometric highly-spin-polarized semiconductor $\mathrm{EuO}$ in close proximity to a highly conductive interfacial layer of oxygen-deficient 
$\mathrm{SrTiO}_{3-\delta}$. The EuO films are ferromagnetic with a Curie temperature of $70 \mathrm{~K}$ and the interfacial 2DEG displays linear positive $\mathrm{MR}$ below the $\mathrm{EuO}$ transition temperature. Using density functional theory, we demonstrate a defect-driven spin-polarized 2DEG at the interface, with the $t_{2 g}$ character of the low dimensional electron system confirmed by resonant SX-ARPES. Combining these results with first-principles calculations, we uncover the role of the spin-polarized oxygen vacancy state as the origin of the linear positive MR, suggesting a path towards developing novel nano-oxide spintronic devices based on strong proximity effects.

This work was supported by the Air Force Office of Scientific Research (FA955012-10494). We thank Chungwei Lin for helpful discussions. 


\section{Chapter 6: Analysis of the Pockels effect in ferroelectric barium titanate thin films on $\mathrm{Si}(001)$}

Contents of this Chapter were published in Microelectron. Eng. 147, 215-218 (2015) [185]. $\S$

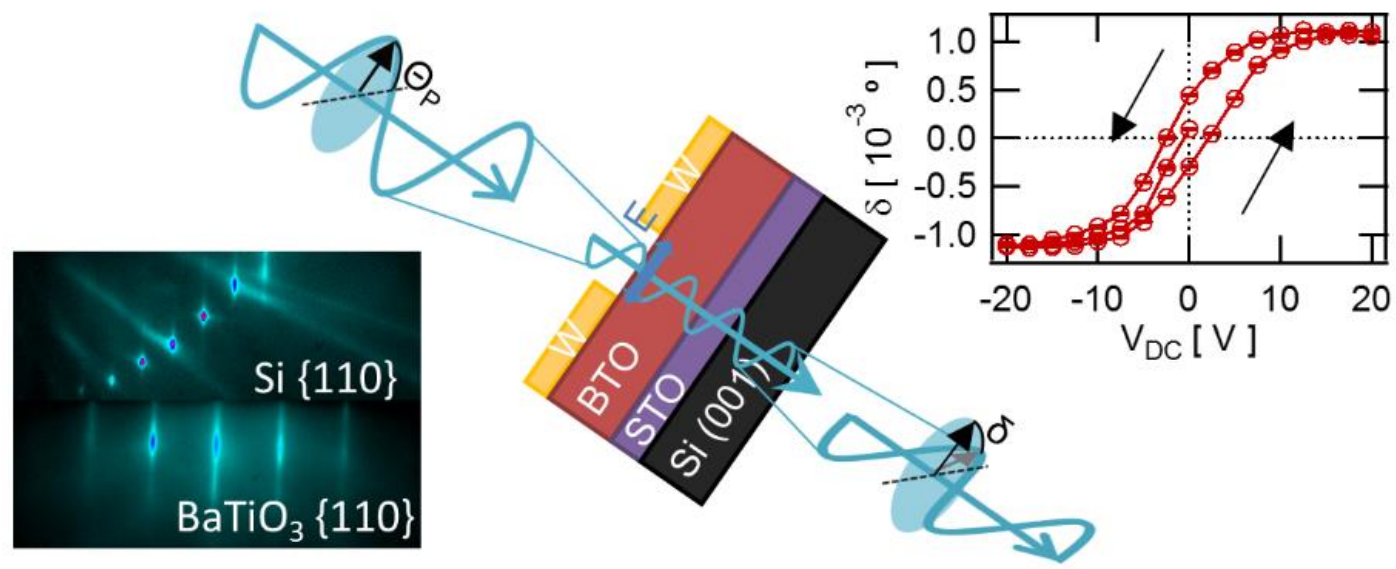

Figure 6.1: Graphical abstract from Ref. [185].

High-quality epitaxial $\mathrm{BaTiO}_{3}$ (BTO) on $\mathrm{Si}$ has emerged as a highly promising material for future electro-optic (EO) devices based on BTO's large effective Pockels coefficient. We report on the EO response of BTO films deposited on Si by molecular beam epitaxy (MBE), and characterize the structure of these films by reflection highenergy electron diffraction and $\mathrm{x}$-ray diffraction. $\mathrm{O}_{2}$ rapid thermal anneal at $600^{\circ} \mathrm{C}$ for 30 min ensures full oxidation of BTO for minimal leakage current with minimal change in crystalline structure.

\footnotetext{
$\S$ Contributions: KJK, ABP, FF, JF, HS, and SA designed and performed the experiments related to the sample growth. KJK, YP, and SA designed, performed, and analyzed the electro-optic measurements. DC performed the lithography steps. KJK, FF, MS, CM, and JF performed the XRD measurements and analyzed the data. AAD, PP, ABP, and LC contributed to the conception and analysis of data. All authors contributed to interpretation of the data and to discussions.
} 


\subsection{INTRODUCTION}

The large effective Pockels coefficient $\left(r_{\mathrm{eff}}=148 \mathrm{pmV}^{-1}\right)$ for high-quality epitaxial BTO films on Si distinguishes BTO as a highly promising material for integrated silicon nanophotonics [67,72]. Monolithic integration of BTO on $\mathrm{Si}$ is enabled by molecular beam epitaxy (MBE) through the use of buffer layers, including $\mathrm{BaO}$ [211], $\mathrm{Ba}_{0.7} \mathrm{Sr}_{0.3} \mathrm{TiO}_{3}$ [212], and most commonly, $\mathrm{SrTiO}_{3}$ (STO) [67,80,213]

Since the first successful integration of perovskite oxides on Si [214] and successive demonstration of ferroelectric functionality [80], electro-optic activity [67], and devices [72], it has become apparent that these interesting properties are strongly dependent on the crystalline structure of ferroelectric BTO. In order to achieve a strong electro-optic response, the film must have (1) the correct crystallographic orientation with respect to the applied electric field [74], and (2) low vacancy density to sustain a strong electric field [215].

In this chapter, we assess BTO films of thickness $40-130 \mathrm{~nm}$ in order to quantify the Pockels coefficient with respect to crystalline orientation. Highly insulating MBEgrown BTO films can be achieved through exposure to oxygen plasma during the deposition or post-deposition $\mathrm{O}_{2}$ rapid thermal anneal (RTA). We also examine the role of oxygen vacancies and structural defects in films of intermediate thickness (80-90 nm).

\subsection{EXPERIMENTAL DETAILS}

Epitaxial BTO films of thickness 40-130 nm were deposited on highly insulating (>20,000 $\Omega$-cm) 2"-diameter Si (001) wafers through use of a Sr Zintl template and 4-6 nm $\mathrm{SrTiO}_{3}$ buffer layer [79], with the growth monitored by in-situ reflection high-energy electron diffraction (RHEED) (Figure 6.2(a)-(c)). 


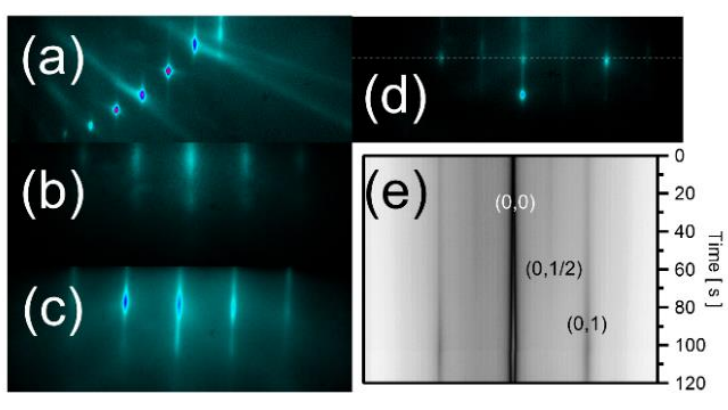

Figure 6.2: RHEED patterns for (a) $\mathrm{Sr}$ Zintl template on Si along Si $\{110\}$, (b) 6 $\mathrm{nm}$ STO along STO $\{110\}$, and (c) $90 \mathrm{~nm}$ BTO along $\{110\}$. (d) The BTO $<210>$ surface unit cell was monitored during growth. An extra streak appears in the RHEED pattern for the Ti-rich surface, indicating a doubling of the surface unit cell size. (e) In a line scan taken during one unit cell of BTO deposition (120 s), the extra streak disappears for stoichiometric BTO.

We note that for the Ti-rich surface, an extra streak appears in the RHEED pattern along the $\{210\}$ direction (Figure 6.2(d)), indicating a doubling of the surface unit cell size. As shown in Figure 6.2(e), this extra streak disappears during the backgroundsubtracted line scan taken during one unit cell of BTO deposition (120 s), indicating stoichiometric BTO. For Ba-rich samples, $\mathrm{BaO}_{\mathrm{x}}$ particles can nucleate at the surface of the as-grown film [216]. As indicated by AFM, soaking in water [217,218] at room temperature for 4 hours reduces the RMS roughness from $0.763 \mathrm{~nm}$ (Figure 6.3(a)) to $0.359 \mathrm{~nm}$ (Figure 6.3(b)).

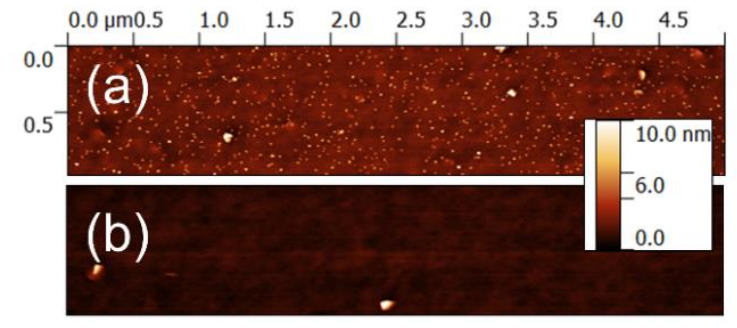

Figure 6.3: Atomic force microscopy (AFM) of the as-grown sample (a) reveals many small particles (presumably $\mathrm{BaOx}$ ) on the surface. After soaking in water for 4 hours (b), few particles remain.

The backside of the Si wafer was polished for optical transmission measurements. A $300 \mathrm{~nm}$-thick tungsten layer was deposited by magnetron sputtering and subsequently dry-etched to define electrode pairs with a gap of $d=5 \mu \mathrm{m}$.

The schematic of EO measurement in transmission geometry for is illustrated in Figure 6.4(a). The in-plane field modulates the refractive index of $\mathrm{BTO}, n(E)=n_{0^{-}}$ 
$(1 / 2) r n_{0}{ }^{3} E$, which, in turn, induces a rotation $(\delta)$ of the incident linearly polarized light. To detect $\delta$, sinusoidal, alternating voltage $\left(E_{A C}=V_{A C} / d\right)$ is applied to gain sensitivity using a lock-in amplifier. An additional DC voltage $\left(E_{\mathrm{off}}=V_{\mathrm{DC}} / d\right)$ is also applied to align the ferroelectric domains.
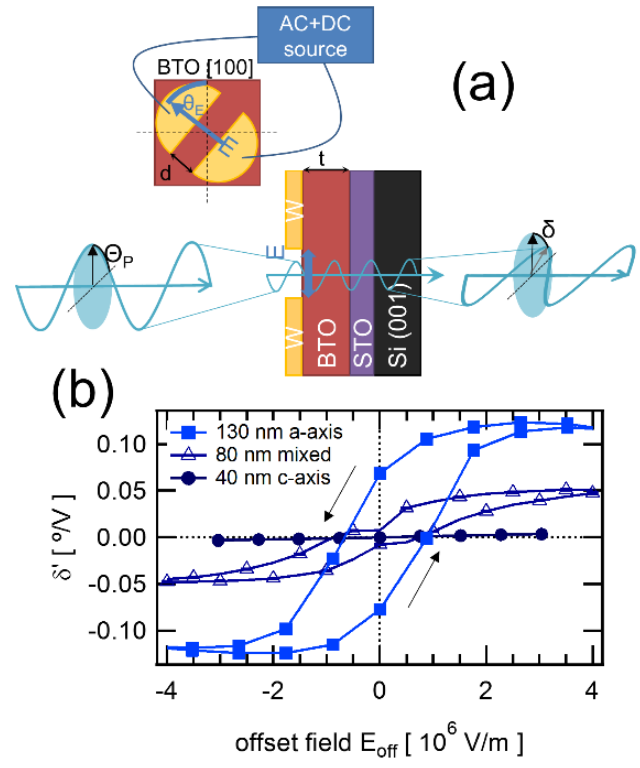

Figure 6.4: (a) Electro-optical characterization quantifies rotation $(\delta)$ of the polarization $\left(\theta_{\mathrm{P}}\right)$ of a laser beam transmitted through pairs of lithographically defined electrodes. (b) Thickness-normalized electro-optic response $\delta^{\prime}=\delta /\left(E_{A C} \cdot t\right)$. as a function of offset (DC) field for a-axis, mixed, and caxis films.

\subsection{RESULTS AND DISCUSSION}

Figure 6.4(b) illustrates the EO

orientation.

Since the magnitude of the response $\delta$ should scale with both the optical interaction length and the magnitude of the applied AC field, we compare the field- and thickness-normalized optical rotation $\delta^{\prime}=\delta /\left(E_{A C} \cdot t\right)$, where $t$ is the thickness of the BTO layer. The thickness of the STO and Si layers are not included, as no Pockels effect is expected for materials with inversion symmetry [219].

The strongest optical response $\delta$ ' is observed when the BTO tetragonal axis is oriented in-plane (" $a$-axis", solid squares). The hysteresis of $\delta$ ' vs DC offset electric field $E_{\text {off }}$ is also characteristic of reorientation of multiple in-plane ferroelectric domains [67]. Alternatively, a vanishing EO response is measured when the BTO tetragonal axis is 
oriented out-of-plane ("c-axis", solid circles). Finally, a strong response is observed even for the "mixed" $a$ - and $c$-axis film (open triangles), attributable to the fraction of $a$-axis domains.

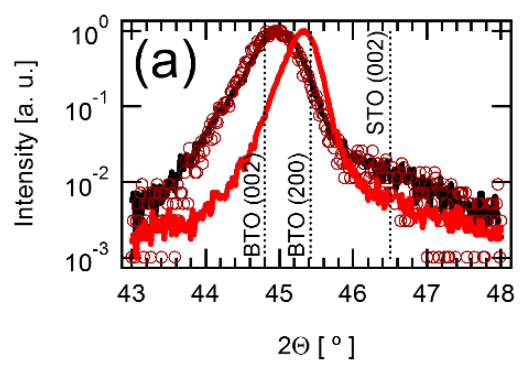

Figure 6.5: (a) Symmetric 2 $\theta-\omega$ scan of a 40-nm BTO film as a sample undergoes a series of 30 min RTA in $\mathrm{O} 2$ at incremental temperatures $\left(500-850^{\circ} \mathrm{C}\right)$. (b)

Corresponding rocking curves about the (002) BTO Bragg peak. (c) Corresponding FWHM and out-of-plane lattice constants over the annealing series

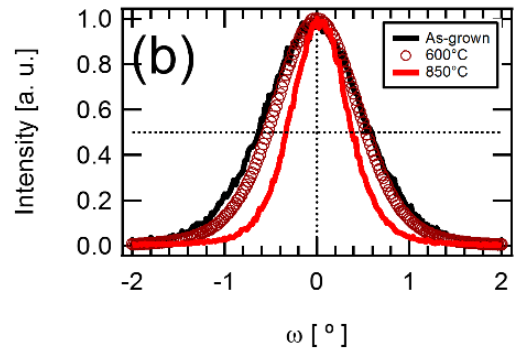

We also identify post-deposition $\mathrm{O}_{2}$ rapid thermal anneal ( $\left.\mathrm{RTA}\right)$ as a route to increase the fraction of $a$-axis BTO. Figure 6.5 illustrates the structural changes

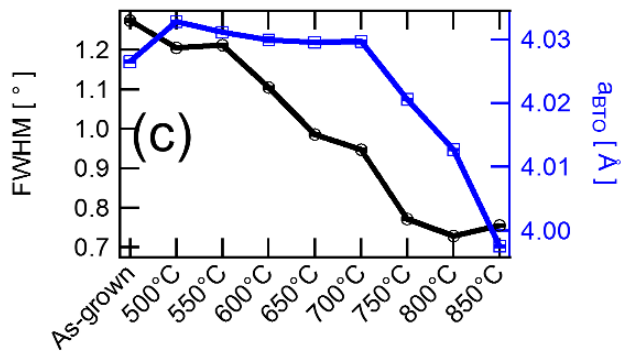
of an as-grown $c$-axis film after successive rounds of RTA $\left(500-850^{\circ} \mathrm{C}, \quad 50^{\circ} \mathrm{C}\right.$ increments, $30 \mathrm{~min}$ each). A reduced FWHM of the (002) BTO Bragg peak rocking curve indicates improved crystallinity. Between the $700^{\circ} \mathrm{C}$ and $850^{\circ} \mathrm{C}$ annealing steps, the out-of-plane lattice constant decreases from $4.03 \AA$ to $3.99 \AA$, indicating a transition from $c$ - to $a$-axis orientation.

An additional role of $\mathrm{O}_{2}$ RTA is the healing of oxygen vacancies, electrically active defects which can impede build-up of a strong electric field. To illustrate, we compare (Figure 6.6) two $90 \mathrm{~nm} \mathrm{BTO} / \mathrm{STO} / \mathrm{Si}$ samples, one deposited in molecular oxygen and the other under oxygen plasma-assisted conditions. The sample as-grown in 
molecular $\mathrm{O}_{2}$ exhibited a high leakage current (Figure 6.6, grey squares). While air anneal up to $300^{\circ} \mathrm{C}$ and RTA at $400^{\circ} \mathrm{C}$ resulted in minimal change, RTA at $600^{\circ} \mathrm{C}$ for 30 min reduced leakage current by 4 orders of magnitude, ensuring a strong electric field and enabling EO measurement (Figure 6.6, black squares). A similarly low leakage current is measured for two as-grown oxygen plasma-assisted samples, one with $\mathrm{Ti}$ evaporated from a Knudsen cell (K-cell) at a rate of $4 \AA /$ min (Figure 6.6, red circles) while the other used an electron-beam evaporated (e-beam) Ti source at a rate of 12 $\AA /$ min (Figure 6.6, blue triangles).

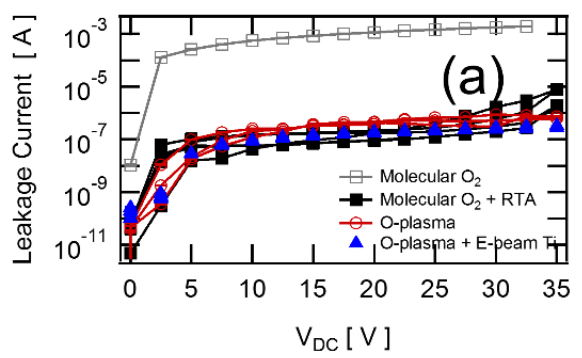

Figure 6.6: (a) Leakage current and (b) symmetric $2 \theta-\omega$ scan for a series of films with varied growth conditions.

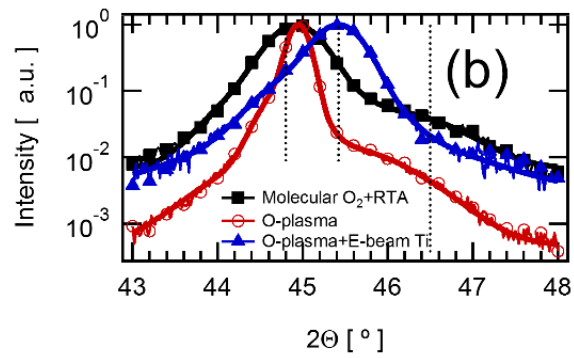

From EO measurements of the three highly insulating samples, we calculate an effective Pockels coefficient, $r_{\mathrm{eff}}=\left(2 \delta_{\max }\right) \lambda /\left(\pi n^{3} t E_{\mathrm{AC}}\right)$, where $\delta_{\max }$ is the maximum induced rotation angle in radians, $\lambda=1550 \mathrm{~nm}$ is the wavelength of incident light, $n=2.26$ is the refractive index of the film as determined by spectroscopic ellipsometry, $t$ the total thickness of the BTO layer [86]. Results are summarized in Table 1 for comparison with previous works, for example $\sim 2 \mathrm{pm} / \mathrm{V}$ for strained $\mathrm{Si}, \sim 30 \mathrm{pm} / \mathrm{V}$ for $\mathrm{LiNbO}_{3}$ on $\mathrm{Si}$, and $148 \mathrm{pm} / \mathrm{V}$ for BTO/Si $[61,67,220]$. By comparing the effective Pockels coefficient for the mixed films, we see that the strongest response is observed for the e-beam Ti sample. XRD of this sample indicates a large fraction of in-plane oriented BTO as compared to the two K-cell Ti samples (Figure 6.6(b)). 


\begin{tabular}{cccccccc}
\hline \hline Sample & $\begin{array}{c}\text { Oxygen } \\
\text { source }\end{array}$ & $\begin{array}{c}\text { Ti } \\
\text { source }\end{array}$ & $\begin{array}{c}\text { Thickness } \\
(\mathrm{nm})\end{array}$ & $\begin{array}{c}\text { Leakage } \\
\text { current }(\mathrm{A})\end{array}$ & $\begin{array}{c}r_{\text {eff }} \\
(\mathrm{pm} / \mathrm{V})\end{array}$ & $\begin{array}{c}\text { FWHM } \\
\left({ }^{\circ}\right)\end{array}$ & $a_{\text {BTO }}(\AA)$ \\
\hline A & Molecular & K-cell & 90 & $10^{-3}$ & N/A & $0.75 \pm 0.07$ & $4.023 \pm 0.008$ \\
A $^{\prime}$ & Anneal & K-cell & 90 & $10^{-7}$ & 46 & $0.79 \pm 0.11$ & $4.031 \pm 0.009$ \\
B & Plasma & K-cell & 90 & $10^{-7}$ & 39 & $0.40 \pm 0.05$ & $4.028 \pm 0.004$ \\
C & Plasma & E-gun & 80 & $10^{-7}$ & 50 & N/A & $3.978 \pm 0.009$ \\
\hline \hline
\end{tabular}

Table 6.1: Pockels coefficients for samples grown under various growth conditions.

\subsection{Conclusion}

In conclusion, for epitaxial $\mathrm{BTO} / \mathrm{Si}$, we measure an increasing $\mathrm{EO}$ response for films with increasing $a$-axis fraction and low leakage current. We further identify postdeposition $\mathrm{O}_{2}$ RTA as beneficial to achieve (1) in-plane tetragonal axis orientation and (2) full oxidation of BTO. Future work should focus on numerical simulations (based on the Pockels tensor, domain structure, field orientation, and angle of incidence) for modeling the response of $c$-axis domains. Such analysis might allow more accurate modeling of the electro-optic response of $c$ - and $a$-axis domains in films with mixed domains to extract the full Pockels tensor of these films.

This work was supported by the National Science Foundation (IRES- 1358111), the European Commission (FP7-ICT-2013-11-619456-SITOGA) and the Air Force Office of Scientific Research (FA9550-12-10494). 


\title{
Chapter 7: Microstructure and ferroelectricity of $\mathrm{BaTiO}_{3} / \mathrm{Si}$ for integrated photonics
}

\author{
Contents of this Chapter were published in Nanotechnology 28, 75706
} (2017) $[221] . * *$

Significant progress has been made in integrating novel materials into silicon photonic structures in order to extend the functionality of photonic circuits. One of these promising optical materials is $\mathrm{BaTiO}_{3}$ or barium titanate (BTO) that exhibits a very large Pockels coefficient as required for high-speed light modulators. However, all previous demonstrations show a noticable reduction of the Pockels effect in BTO thin films deposited on silicon substrates compared to BTO bulk crystals. Here, we report on the strong dependence of the Pockels effect in BTO thin films on their microstructure, and provide guidelines on how to engineer thin films with strong electro-optic response. We employ several deposition methods such as molecular beam epitaxy and chemical vapor deposition to realize BTO thin films with different morphology and crystalline structure. While a linear electro-optic response is present even in porous, polycrystalline BTO thin films with an effective Pockels coefficient $r_{\text {eff }}=6 \mathrm{pm} / \mathrm{V}$, it is maximized for dense, tetragonal, epitaxial BTO films $\left(r_{\text {eff }}=140 \mathrm{pm} / \mathrm{V}\right)$. By identifying the key structural predictors of electro-optic response in $\mathrm{BTO} / \mathrm{Si}$, we provide a roadmap to fully exploit the linear electro-optic effect in novel hybrid oxide/semiconductor nanophotonic devices.

\footnotetext{
** Contributions: KJK, YP, and SA designed, performed, and analyzed the electro-optic measurements. KJK, MS, and CM performed the XRD measurements and analyzed the data. MS designed and analyzed the ellipsometry measurements. Simulations and data analysis were done by KJK and YP with support from SA. AAD JF and SA contributed to the conception and analysis of data. JF prepared the MBE sample, SA and DC prepared the sputtered samples, MT prepared the PLD sample with support from MF, and MR prepared the CVD sample with help from PH. DC performed the lithography steps. FE, MS, and MDR performed the STEM experiments and KJK analyzed the data. YP performed COMSOL simulations. All authors contributed to interpretation of the data and to discussions.
} 


\subsection{INTRODUCTION}

For decades, increasing the efficiency and performance of information processing units has been mainly driven by reducing the size of transistors. However, since the scaling law approaches a natural limit, novel concepts such as the introduction of highmobility materials as transistor channels or optical links for intra-chip communications have more recently been actively investigated. Indeed, silicon photonics offers low-cost fabrication of high-bandwidth and low-power data transmission techniques beyond what is offered by ordinary electrical connections. $[55,56,60,62,71]$ The technology has been boosted by the development of novel components including silicon passives and germanium detectors, but it still lacks some essential device types such as highperforming optical modulators. Since these components have been mastered in bulk telecommunication applications by the usage of materials with strong electro-optical properties such as lithium niobate, [50] a concerted effort has emerged to integrate similar materials into silicon photonic structures, where the refractive index index $n$ can be expressed in terms of the static external electric field $E$, [222]

$$
n(E)=n_{0}-\frac{1}{2} n_{0}^{3} r E
$$

where $n_{0}$ is the zero-field value of the refractive index and $r$ is the Pockels coefficient. In particular, the recent integration of barium titanate $\left(\mathrm{BaTiO}_{3}\right)$ on silicon with large Pockels coefficients $[63,67,72,185]$ opens an exciting opportunity for designing and realizing high-speed modulators, and novel device types such as nonvolatile optical memories. The latter elements would be of great interest as synaptic elements for optical neural networks. [64]

Despite the recent integration success, the linear electro-optic effect in $\mathrm{BaTiO}_{3}$ (BTO) thin films determined in previous experiments clearly shows deteriorated 
properties compared to bulk BTO crystals. $[63,67,72,185]$ From a materials perspective, understanding the mechanism of this degradation opens exciting opportunities to further engineer and tailor the Pockels coefficients in thin films. We have developed and described in detail a characterization technique which allows us to determine the main tensorial properties of the Pockels effect and to demonstrate BTO ferroelectric switching by means of optical measurements. [67] We have formulated the systematic design rules to tailor deposition parameters and obtain high quality crystalline BTO layers on Si substrates. [223]

In this chapter, we demonstrate experimentally how the crystalline quality and film morphology of the active material impact the magnitude of the Pockels effect. To compare and contrast layers of widely varied properties, we deposited $\sim 100 \mathrm{~nm}$ thin BTO films on silicon substrates using several different deposition methods. By analyzing the structural and electro-optic characteristics of the layers, we identified key structural predictors of a large Pockels coefficient. In particular, reducing the porosity and increasing the crystalline grain size are the key contributors to maximizing the electrooptic response. Our study outlines the path for material scientists to design highly efficient, novel hybrid silicon photonic devices based on nanoscale oxides integrated on silicon.

\subsection{EXPERIMENTAL DETAILS}

\subsubsection{Sample preparation}

In order to realize different layer properties, we deposited BTO thin films via molecular beam epitaxy (MBE), pulsed laser deposition (PLD), chemical vapor deposition (CVD), and radio-frequency sputtering (RF sputtering). Except when explicitly mentioned, we used a 4-nm-thin $\mathrm{SrTiO}_{3}$ (STO) seed layer grown epitaxially by 
MBE on highly-resistive $(>20,000 \Omega \cdot \mathrm{cm})$ double-side-polished Si (001) wafers for all successive BTO deposition. Details of the STO deposition can be found elsewhere. [224]

Subsequently, BTO was fabricated by MBE (MBE-BTO) in a layer-by-layer deposition process. Therefore, layers of titanium and barium were iteratively deposited in oxygen atmosphere ( $\mathrm{p} \sim 10^{-5}$ mbar) keeping the substrate at $\sim 600^{\circ} \mathrm{C}$. Details of the deposition process are described in Ref. [67]. The PLD thin films (PLD-BTO) were grown at a substrate temperature of $690^{\circ} \mathrm{C}$ under an oxygen pressure of 0.15 mbar using an excimer $\mathrm{KrF}$ laser. The laser repetition rate was set to $2 \mathrm{~Hz}$ and the fluence at $1.2 \mathrm{Jcm}^{-}$ ${ }^{2}$. For CVD deposition of BTO the heated substrate $\left(400^{\circ} \mathrm{C}\right)$ was exposed to barium isopropyl cyclopentadienyl, titanium isopropoxide and water as reactive precursors in a high vacuum $\left(10^{-6}\right.$ mbar $)$ environment; comparable to the process described in Ref. [225]. Two additional samples of BTO were prepared by RF sputtering. The first utilized an 8-nm-BTO/4-nm-STO/Si pseudosubstrate prepared by MBE as discussed above, while the second was sputtered directly on Si with no buffer layer using the same parameters as described in Ref. [223]. After the sputter deposition at $500^{\circ} \mathrm{C}$, the samples were annealed in oxygen at $650^{\circ} \mathrm{C}$ for 20 minutes to ensure full crystallization.

\subsubsection{Structural characterization}

For each of the samples, we extracted the lattice parameters for the BTO film via both out-of-plane and grazing incidence in-plane X-ray diffraction (XRD) measurements using a Bruker AXS D8 Discover. Out-of-plane and in-plane scans are aligned to Si (004) and (220) peaks, respectively. Atomic force microscopy analysis was performed using a Veeco Dimension V. The refractive index, thickness, and porosity of the BTO films have been characterized by spectroscopic ellipsometry using a Variable Angle Spectroscopic 
Ellipsometer VASE® from J.A. Woollam Co. Data have been acquired for wavelengths between $300 \mathrm{~nm}$ and $1200 \mathrm{~nm}$ at three angles of incidence $\left(65,70\right.$ and $\left.75^{\circ}\right)$.

Cross sectioning and lamella preparation were carried out by means of a FEI Helios Nanolab 450S focused ion beam. Bright-field and medium-angle annular dark field (MAADF) scanning transmission electron microscopy (STEM) measurements were performed using a double spherical aberration-corrected JEOL JEM-ARM200F microscope operated at $200 \mathrm{kV}$ with a probe convergence semiangle set to $25.3 \mathrm{mrad}$. The annular semi-detection range of the annular dark-field detector was calibrated at 40$160 \mathrm{mrad}$ for the MAADF images. For the bright-field images, the outer semi-detection range of the bright-field detector was set to $45 \mathrm{mrad}$.

\subsubsection{Electro-optic characterization}

We determined the Pockels coefficients by analyzing the change of the polarization of a laser beam transmitted through the BTO film while applying an electric field. In order to generate such a field, pairs of 300-nm-thick tungsten electrodes with the electrode gap $d=5 \mu \mathrm{m}$ were defined by optical lithography and $\mathrm{SF}_{6} / \mathrm{N}_{2}$ reactive ion etching. In order to assess the tensorial nature of the Pockels effect, we varied the angle $\theta_{\mathrm{E}}$ between the electric field and the BTO crystalline axis by fabricating differently oriented electrode pairs, as defined in Figure 7.1 (a).

The electro-optic characterization follows the principle illustrated in Figure 7.1(b)-(c) using the Sénarmont set up described in detail in Section 2.3 and elswehere. [67] A linearly polarized New Focus diode laser model 6262 with wavelength $1550 \mathrm{~nm}$ focused to a spot size of $\sim 30 \mu \mathrm{m}$ full-width at half-maximum was first aligned

to the electrode gap. A half-wave plate was used to set the incident polarization. Due to 
the birefringence of BTO, the sample introduces a phase shift between orthogonal polarization components of the incident light.

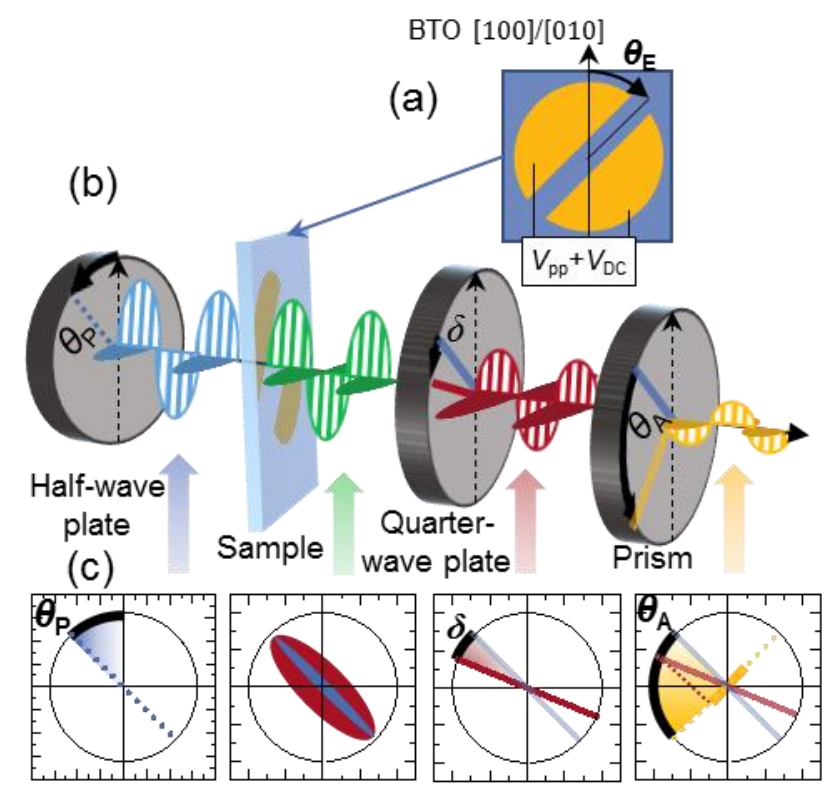

Figure 7.1: (a) In-plane electrode pairs were patterned with various angles $\theta_{\mathrm{E}}$ defined relative to the BTO $[100] /[010] /[001]$ direction. As described in the text, the sinusoidal measuring and static poling voltages $V_{\mathrm{pp}}+V_{\mathrm{DC}}$ are applied across the electrodes. (b) For electro-optic transmission measurements, a half-wave plate is used to set the incident linear polarization. After passing through the sample, the light becomes elliptically polarized, which is transformed into linearly polarized light after passing a quarter-wave plate. Finally, a Glanlaser prism is used to determine the orientation of the transmitted polarization. (c) Corresponding states of the polarization along the path of the laser beam are visualized as the ellipse traced out by the light's electric field vector with (red) and without (blue) an external electric field. After transmission through the sample and quarter-wave plate, the polarization is rotated by angle $\delta$.

This phase shift is compensated by a quarter-wave plate (QWP), whose orientation is determined by iteratively rotating the QWP and a successive Glan-laser prism to minimize the transmitted power. After this alignment process, the light after the QWP is linearly polarized. In that configuration, a change of the birefringence in the 
BTO layer results in a rotation of the polarization after the QWP by an angle $\delta$ with respect to the zero-field state.

For increased sensitivity, a lock-in amplifier system was used to isolate the rotation $\delta$ induced by a sinusoidal AC field modulated at frequency $f=17.3 \mathrm{kHz}$. Typical values of the peak-to-peak $\mathrm{AC}$ voltage $V_{\mathrm{PP}} \sim 3 \mathrm{~V}$ were much smaller than the typical voltage used to align ferroelectric domains $V_{\mathrm{DC}} \sim 20 \mathrm{~V}$. Since in the lock-in configuration only the rotation $\delta$ corresponding to the AC modulation is measured, we introduce a field-normalized rotation, $\delta^{\prime}=\delta / E_{A C}$, where the root mean square AC field was defined as $E_{A C}=\mathrm{V}_{\mathrm{PP}} /(2 \sqrt{2} d)$ and $\delta$ is the rotation defined in Figure 7.1. The static offset field applied to align ferroelectric domains was defined as $E_{\mathrm{off}}=V_{\mathrm{DC}} / d$, where $d$ is the gap between electrodes. While the magnitude of this offset field does not enter directly into the definition of the Pockels coefficient, it does influence the fraction of poled domains $v$. In the presence of polarization-reversed domains as in an un-poled film, a reduced response could be measured due to cancellation of contributions from antiparallel domains, leading to underestimated Pockels coefficients. [85] For this reason, Pockels coefficients reported here are for poled films, corresponding to $v=1$ at at $\theta_{\mathrm{E}} \sim 45^{\circ}$ and $v$ $=0.5$ at at $\theta_{\mathrm{E}} \sim 90^{\circ}$ as justified in the following sections.

The effective electro-optic coefficient can then be defined as follows,

$$
r_{\text {eff }}=\Gamma \lambda /\left(v \times E_{A C} \times \pi \times n_{B T O}^{3} \times t\right),
$$

adapted from Ref. [86], where $\Gamma=2 \delta$ is the induced phase shift between orthogonal polarization components, $\lambda=1550 \mathrm{~nm}$ is the wavelength of the transmitted light, $v$ is the net fraction of poled BTO domains, $E_{\mathrm{AC}}$ is the measuring field defined above, $n_{\mathrm{BTO}}$ is the refractive index of the film, and $t$ is the thickness of the BTO layer. As described in detail elsewhere, [67] the rotation of polarization $\delta$ depends strongly on the orientation of the electric field. To capture this dependence and fully describe the Pockels 
effect, we report both the effective Pockels coefficient $r_{\text {eff }}$ and the $c$-axis Pockels coefficient $r_{\mathrm{c}}$ for each sample. Specifically, we extract $r_{\mathrm{eff}}$ from measurements at $\theta_{\mathrm{E}} \sim$ $45^{\circ}$ assuming $v=1$ and $r_{\mathrm{c}}$ from measurements at $\theta_{\mathrm{E}} \sim 90^{\circ}$ assuming $v=0.5$. [67] Full dependence of these coefficients on the Pockels tensor elements are discussed in Refs. [67] and [85]. In order to extract Pockels tensor elements in addition to these effective values, numerical simulations based on the Pockels tensor and domain structure were carried out to best approximate $\delta$ for all measured electrode angles $\theta_{\mathrm{E}}$.

To accurately represent error bars, several known sources of error, including stability over time, position of the laser within the gap, and angle of incidence, were analyzed: Less than $4 \%$ uncertainty originates from experimental error sources. In addition, data were recorded for six sets of similar electrodes with $\theta_{\mathrm{E}} \sim 45^{\circ}$ for each sample in order to account for pad-to-pad variations originating from materials inhomogeneity. The combined errors are reported later in the chapter.

\subsection{RESULTS}

\subsubsection{Structural Characterization}

The strong Pockels effect in BTO originates from the peculiarity of the Ti-O bond that results in a combination of a non-centrosymmetric crystal lattice of the tetragonal phase and an anharmonic potential. [226] Therefore, defects that break the periodicity of the lattice and thus affect long wave length phonons or that influence the crystalline symmetry are expected to diminish the electro-optic response. Examples of the impact of defects on the structural and functional properties of BTO are the stabilization of BTO in the cubic phase for polycrystalline layers with nanometer-sized grains, [223] strain enhancement of the ferroelectric polarization, [227] and relaxor behavior under stoichiometry deviation. [228] Hence, in the following we first discuss in detail the films' 
morphology and crystalline structure, before analyzing the electro-optic results and correlating both the structural and the functional features.

(a) MBE

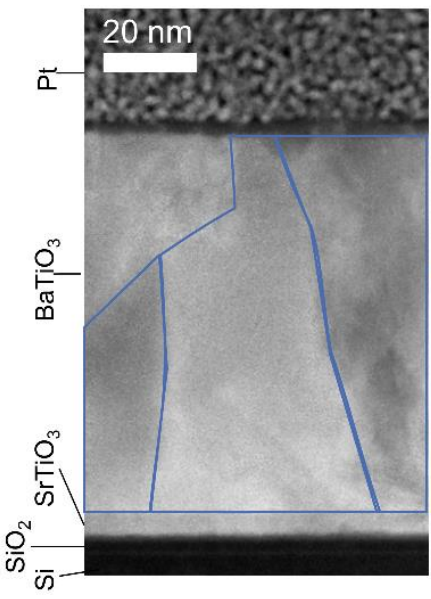

(b) PLD

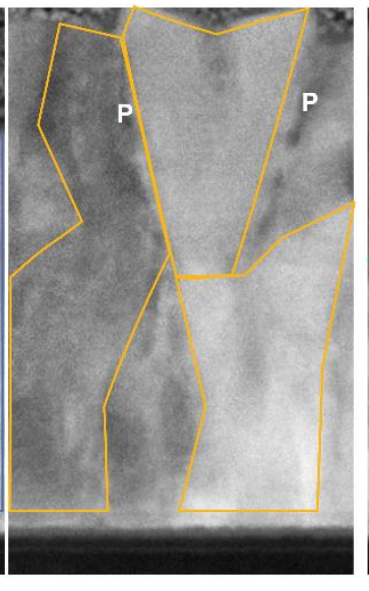

(c) RF Sputter

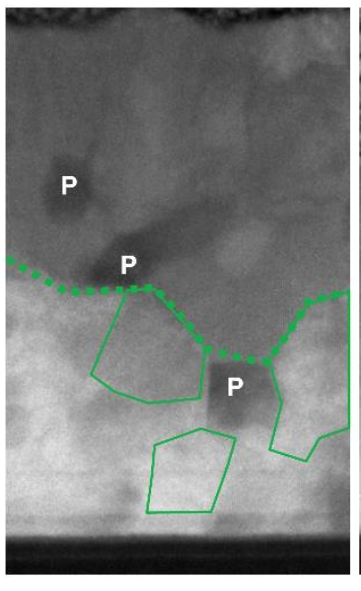

(d) CVD

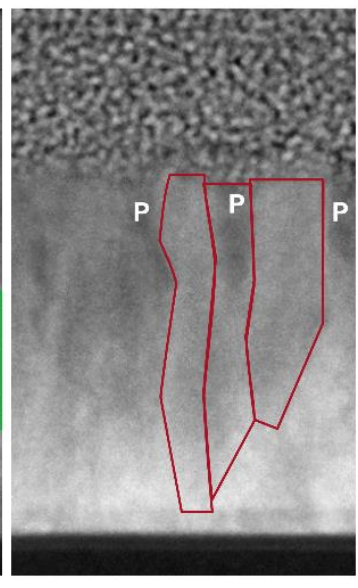

Figure 7.2: (a) Medium-angle annular dark-field scanning transmission electron microscopy (MAADF-STEM) images of BTO thin films deposited by MBE, (b) PLD, (c) RF sputtering, and (d) CVD. Pores are labelled "P". The boundaries of representative grains are outlined with solid lines. In (c), the boundary between epitaxial and polycrystalline BTO is indicated with dashed line.

We determined the porosity and the thickness of the BTO layers by simulating the spectroscopic ellipsometry data with a Bruggeman effective medium approximation model. In this model, we assume the films to consist of a BTO matrix, having the refractive index of bulk BTO along its ordinary axis [229] and a fraction of spherical voids, having the refractive index of air (1.0). The porosity is quantified as the volume fraction $p$ of the voids. In order to reduce the number of fit parameters we neglect the dependence of the refractive index of the BTO matrix on other structural parameters such as grain boundaries, stoichiometry, strain, and crystallographic orientation. This simplification allows us to consistently evaluate the porosity and thickness for all five 
samples (Table 7.1). The BTO film thickness extracted from the spectroscopic ellipsometry model and compared with the MAADF-STEM images (Figure 7.2(a)-(d)) only show slight deviations of $<5 \%$. To account for this difference, we determined the uncertainty of $p$ by intentionally varying the thickness of the BTO layer by $\pm 5 \%$ around the value initially determined by spectroscopic ellipsometry (Table 7.1). We could qualitatively verify the large difference in porosity between the films as determined by spectroscopic ellipsometry with our bright-field-STEM analysis, where pores are clearly visible as areas of decreased density (Figure 7.2 and Appendix B, Figure B.1). The different surface roughness among the samples is also in agreement with the varying porosity. In particular, the atomic force microscopy of the dense MBE-BTO layer ( $p=$ $3 \%$ ) shows a low root mean square roughness of only $\sim 0.5 \mathrm{~nm}$ (Appendix B, Figure B.2).

\begin{tabular}{|c|c|c|c|c|c|c|}
\hline BTO Method & Buffer Layer & $\bar{n}$ & $p[\%]$ & ]$^{t[\mathrm{~nm}}$ & Texture & $e d r[\mathrm{~nm}]$ \\
\hline MBE & $4 \mathrm{~nm}$ MBE STO & 2.27 & $3( \pm 2)$ & 78 & Epitaxial & $21( \pm 6)$ \\
\hline PLD & $4 \mathrm{~nm}$ MBE STO & 2.18 & $14( \pm 3)$ & 97 & Epitaxial & $21( \pm 4)$ \\
\hline RF Sputter & $\begin{array}{c}4 \mathrm{~nm} \text { MBE STO } \\
+8 \mathrm{~nm} \mathrm{MBE} \\
\text { BTO }\end{array}$ & 2.21 & $11( \pm 3)$ & 96 & $\begin{array}{c}\text { Epitaxial } \\
+ \\
\text { Polycrystalline }\end{array}$ & $10( \pm 2)$ \\
\hline CVD & $4 \mathrm{~nm}$ MBE STO & 2.13 & $21( \pm 1)$ & 70 & Epitaxial & $14( \pm 1)$ \\
\hline $\begin{array}{l}\text { RF Sputter (no } \\
\text { buffer) }\end{array}$ & -- & 2.12 & $21( \pm 3)$ & 102 & Polycrystalline & -- \\
\hline
\end{tabular}

Table 7.1: Summary of film microstructure parameters. From ellipsometry, we extract the refractive index $n$ of the BTO matrix, porosity volume fraction $p$ and thickness of the BTO layer $t$. Error bars of porosity correspond to a BTO thickness variation of 5\% (details in the text). From STEM, $e d r$ is the average equivalent disc radius of the grains highlighted in Figure 7.2.

With an epitaxial STO buffer layer, the epitaxial relationship of BTO to the Si substrate could be preserved even as an amorphous $\mathrm{SiO}_{\mathrm{x}}$ interfacial layer was formed during deposition or post-anneal (Figure 7.2), in agreement with previous 
studies. [67,80,223] However, an STO buffer layer might not always prevent the formation of some randomly oriented domains. [223] Indeed, we observe a transition from epitaxial to polycrystalline orientation in the RF sputtered BTO film (Figure 7.2(c), dashed line). As expected, the film sputtered directly on Si without any buffer layer is fully polycrystalline. [223]

In MAADF-STEM images (Figure 7.2), the contrast is sensitive to diffraction effects. Accordingly, the crystal lattice of bright grains is oriented along the electron beam, and homogenous brightness indicates homogenous crystalline orientation. Additional contrast modulation, as in Figure 7.2(a), originates from projecting a 3D domain structure of multiple grains into a 2D image. As a guide to the eye, we highlighted several grains per image in Figure 7.2. We estimated the grain size by calculating the equivalent disc radius $e d r$ via $e d r=\sqrt{A / \pi}$ (Table 7.1) where $A$ corresponds to the area of the highlighted grains. While columnar grains with $e d r>20 \mathrm{~nm}$ were observed for BTO deposited by MBE and PLD (Figure 7.2(a) and (b)), smaller grains of $e d r<15 \mathrm{~nm}$ were observed for BTO deposited by CVD and RF sputtering (Figure 7.2(c) and (d), Table 7.1).

XRD was employed to quantify tetragonality, a signature of symmetry breaking, of the BTO films. In the ferroelectric phase at room temperature, bulk BTO shows a noncentrosymmetric tetragonal $P 4 \mathrm{~mm}$ symmetry with two distinct lattice parameters, $a$ and $c$. The tetragonal distortion for bulk crystals is $\gamma=c / a-1=1 \%$. [230] Previous studies of MBE-grown BTO films deposited on STO-buffered Si have shown a multi-domain structure: While thin films are single-domain with the long $c$-axis oriented perpendicular to the interface, a transition occurs for thicker films, [80,223] with a mixture of domains with the $c$-axis oriented perpendicular (along (001)) and parallel to the surface (along (100)/(010)). 


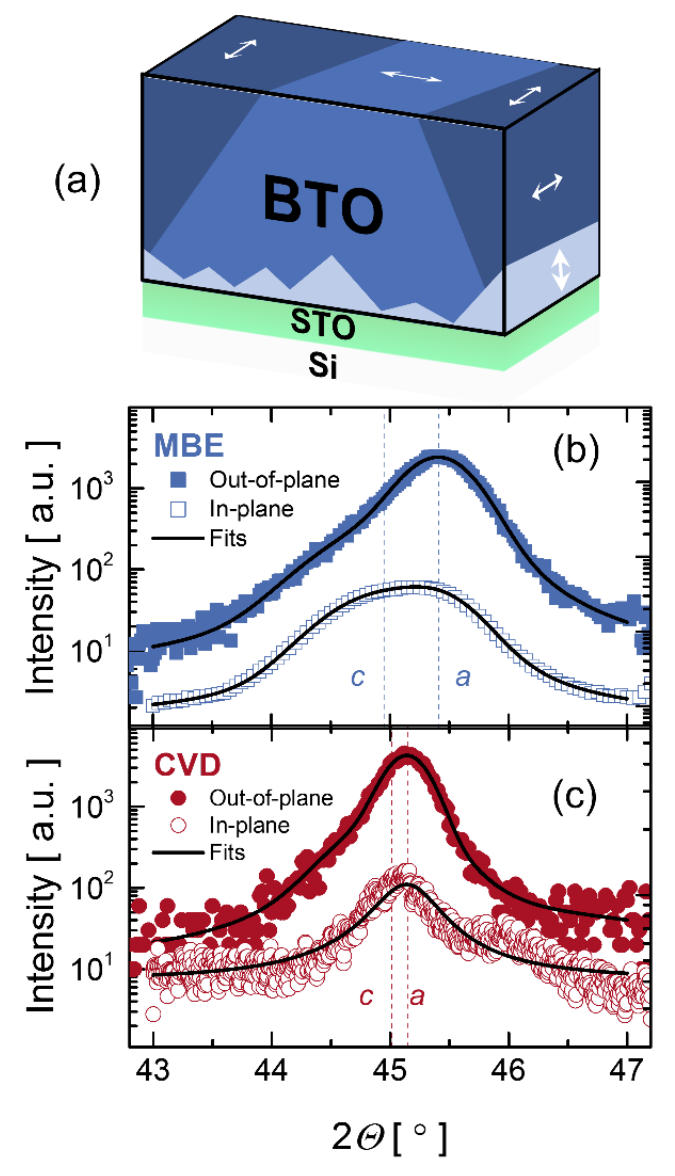

Figure 7.3 (a) Schematic of $90^{\circ}$ domain structure, with the BTO tetragonal axes indicated by white arrows. (b) X-ray diffraction patterns for different measurement configurations for epitaxial BTO deposited by MBE and (c) CVD. A sum of 3 Voigt functions has been fitted to each pair of scans (solid lines), with vertical dashed lines indicating the positions of the peaks corresponding to the BTO $a$ and $c$ lattice parameters.

Using this "mixed model" of the domain structure (Figure 7.3(a)) we determined both the $a$ and $c$ lattice parameters from the XRD data. Therefore, we fit each pair of out-of-plane and inplane XRD scans simultaneously as the sum of three Voigt functions with shared peak parameters: Two components correspond to the (001)-oriented and (100)/(010)-oriented domains of BTO, with a third component corresponding to cubic STO. The STO component is only applied in samples with STO buffer layer. Figure 7.3 (b) and (c) illustrate fits to the BTO (002) and (200)/(020) peak of samples deposited by MBE (MBE-BTO) and CVD (CVD-BTO), respectively, with corresponding bulk lattice positions indicated by vertical dashed lines. The tetragonality of the CVD-BTO film is significantly reduced compared to the MBEBTO film. Lattice parameters and tetragonal distortion $\gamma$ for all samples are summarized in Table 7.2. The XRD analysis also confirms the epitaxial relationship between BTO and Si in all samples (Appendix B, Figure B.3(a)). Only sputtered BTO layers show (110) reflections, indicating a small fraction of polycrystalline domains if a STO seed 
layer is used and a fully polycrystalline film if no STO layer was deposited. The analysis of the misorientation of crystalline domains assessed via rocking curve measurements of the BTO (002) reflection does not show strong differences among the films, as it is essentially determined by the mosaicity of the underlying STO layer. The rocking curve full-width at half maximum for all samples is within a similar range of $1.2^{\circ}$ to $2.1^{\circ}$ (Appendix B, Figure B.3(b)), where the lower and upper bounds correspond to films deposited by PLD and MBE, respectively.

\begin{tabular}{cccc}
\hline \hline BTO Method & Peak & $a / c[\AA]$ & $\gamma[(\%]$ \\
\hline MBE & $(002)$ & $3.99 / 4.03$ & $1.0( \pm 0.1)$ \\
PLD & $(002)$ & $4.00 / 4.02$ & $0.6( \pm 0.1)$ \\
RF Sputter & $(002)$ & $3.99 / 4.05$ & $1.7( \pm 0.1)$ \\
CVD & $(002)$ & $4.01 / 4.02$ & $0.3( \pm 0.3)$ \\
RF Sputter (no buffer) & $(110)$ & $4.03 / 4.04$ & $0.2( \pm 0.4)$ \\
\hline \hline
\end{tabular}

Table 7.2: Results from XRD analysis of $c$ and $a$ lattice parameters for BTO $P 4 \mathrm{~mm}$ symmetry. Tetragonality $\gamma=c / a-1$. Error bars correspond to uncertainty of fit parameters.

Observing a variation of film density, crystallinity, surface roughness, texture (grain orientation and size), and tetragonality among differently fabricated samples is consistent with observations that film microstructure varies with deposition method, [231] temperature and growth rate [232-234], post-deposition treatments [235], strain [236], and stoichiometry . [237] The variation in the BTO films discussed here cannot be used to generally evaluate the different deposition techniques, as none of the deposition has been thoroughly optimized. [238,239] In this chapter, we instead leverage the range of crystalline quality to determine the predictors of electro-optic response. 


\subsubsection{Electro-optic Characterization}

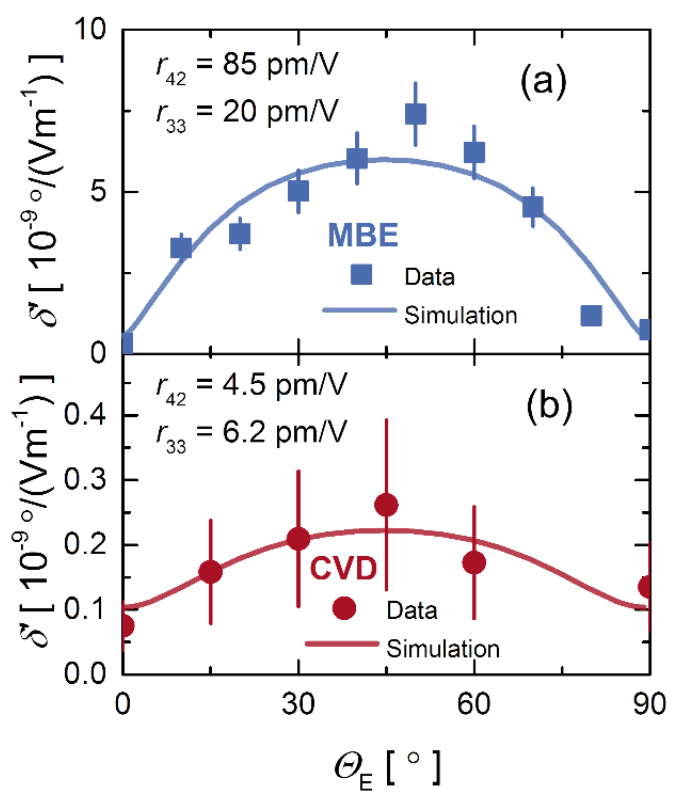

Figure 7.4: (a) The field-normalized induced rotation $\delta$ ' was measured (symbols) and simulated (lines) for varied orientation angle $\theta_{\mathrm{E}}$ (defined in Figure 7.1(a)) of in-plane electric field for BTO films deposited by MBE and (b) CVD. Details on error bars can be found in the methods section.

We provide effective Pockels coefficients $r_{\text {eff }}$ and the $c$-axis coefficient $r_{\mathrm{c}}$ for all samples in Table 7.3 according to Eq. (7.2) for comparison with the literature values and for evaluating device

applications. Due to the random domain orientation, we can only extract $r_{\text {eff }}$ but no individual tensor elements for the fully polycrystalline film. The Pockels coefficients strongly vary among the different samples (Table 7.3), which is for example directly visible in the comparison of the electro-optic response between MBE-BTO and CVDBTO (Figure 7.4).

The dependence of $\delta$ ' on the orientation angle $\theta_{\mathrm{E}}$ of in-plane electric field matches our simulations as shown in the examples for MBE-BTO and CVD-BTO (Figure 7.4). Data for all films are available in Appendix B, Figure B.4. Following the procedure described in the Appendix B, the polarization for light transmitted through a sample can be simulated based on the Pockels tensor for the space group P4mm, taking thickness values from ellipsometry and assuming bulk values for the refractive indices and birefringence. Because domains with the $c$-axis oriented out-of-plane of the film do not contribute to changes in transmission in our measurement geometry, we assume only in- 
plane $90^{\circ}$ domains for our model in order to report a lower bound for the Pockels coefficients. By simulating the response of the samples for the specific measurement geometry used in our experiments, we extracted the best fit Pockels tensor elements $r_{42}$ and $r_{33}$ for each BTO sample (Table 7.3). Because our geometry does not permit independent measurement of $r_{33}$ and $r_{13}$, we assume the bulk ratio of $r_{33}=10 \times r_{13}$. [240]

\begin{tabular}{ccccc}
\hline \hline BTO Method & $\left.\left.\begin{array}{c}r_{42} \\
{[\mathrm{pm} / \mathrm{V}]}\end{array}\right] \begin{array}{c}r_{33} \\
{[\mathrm{pm} / \mathrm{V}]}\end{array}\right] \begin{array}{c}r_{\text {eff }} \\
{[\mathrm{pm} / \mathrm{V}][\mathrm{pm} / \mathrm{V}]}\end{array}$ \\
\hline MBE & $85( \pm 0.6)$ & $20( \pm 0.4)$ & $140( \pm 18)$ & $20( \pm 3)$ \\
PLD & $26( \pm 0.2)$ & $16( \pm 0.3)$ & $37( \pm 27)$ & $14( \pm 11)$ \\
RF Sputter & $27( \pm 0.2) 5.1( \pm 0.1)$ & $41( \pm 15)$ & $7( \pm 3)$ \\
CVD & $4.5( \pm 0.1) 6.2( \pm 0.1)$ & $7( \pm 4)$ & $5( \pm 3)$ \\
RF Sputter (no buffer) & N/A & N/A & $6( \pm 1)$ & $3( \pm 1)$ \\
\hline \hline
\end{tabular}

Table 7.3: Summary of best-fit Pockels tensor elements $r_{42}$ and $r_{33}$ for each BTO sample. Effective Pockels coefficient $r_{\text {eff }}$ and $c$-axis coefficient $r_{\mathrm{c}}$ are calculated according to Eq. (7.2). Error bars for $r_{42} r_{13}$ were estimated by repeating the fitting procedure for $n_{\mathrm{b}}=0.02$ and 0.04 . Error bars for $r_{\mathrm{eff}}$ and $r_{\mathrm{c}}$ correspond to pad-to-pad variation determined from multiple measurements of the $\theta_{\mathrm{E}}=$ $45^{\circ}$ orientation for each sample.

For the determination of the Pockels tensor elements, we also need to take into account the overall ferroelectric domain structure. We evaluate the hysteresis by measuring $\delta$ ' as a function of the offset field $E_{\text {off }}$ for a fixed value $\theta_{\mathrm{E}} \sim 45^{\circ}$, where $\delta$ ' and hence the resolution of the experiment is maximized (Figure 7.5, and Appendix B, Figure B.5). [67] As described previously, [67] on the macroscopic scale of our gap size, $\delta^{\prime}$ ' is generally zero for the as-deposited film, reaches a finite remanence value ${ }^{\delta_{r}^{\prime}}=\delta^{\prime}(0)$ after poling with sufficiently large electric fields, and saturates at a maximum rotation of $\delta$ s'. These values are indicated in Figure 7.5, and Appendix B, Figure B.5. We can then calculate the remanence ratio or "squareness" factor $\delta_{r}^{\prime} / \delta^{\prime}{ }_{s}$, where a ratio of 1 
corresponds to a perfectly square loop, and $E_{\mathrm{c}}$ is the coercive field for each sample (Table 7.4).

\begin{tabular}{ccc}
\hline \hline BTO Method & $\delta^{\prime}{ }_{r} / \delta^{\prime}{ }_{s} E_{\mathrm{c}}$ \\
& {$[\%]$} & {$[\mathrm{kV} / \mathrm{cm}]$} \\
\hline MBE & $35( \pm 3)$ & $7( \pm 0.4)$ \\
PLD & $34( \pm 2)$ & $18( \pm 1)$ \\
RF Sputter & $10( \pm 5)$ & $4( \pm 2)$ \\
CVD & $4( \pm 4)$ & $2( \pm 2)$ \\
RF Sputter (no buffer) & $5( \pm 4)$ & $4( \pm 3)$ \\
\hline \hline
\end{tabular}

Table 7.4: Summary of parameters related to ferroelectricity from electro-optic measurements, remanence ratio $\delta_{r}^{\prime} / \delta^{\prime}{ }_{s}$ and coercive field $E_{\mathrm{c}}$. Error bars correspond to uncertainty of fit parameters.

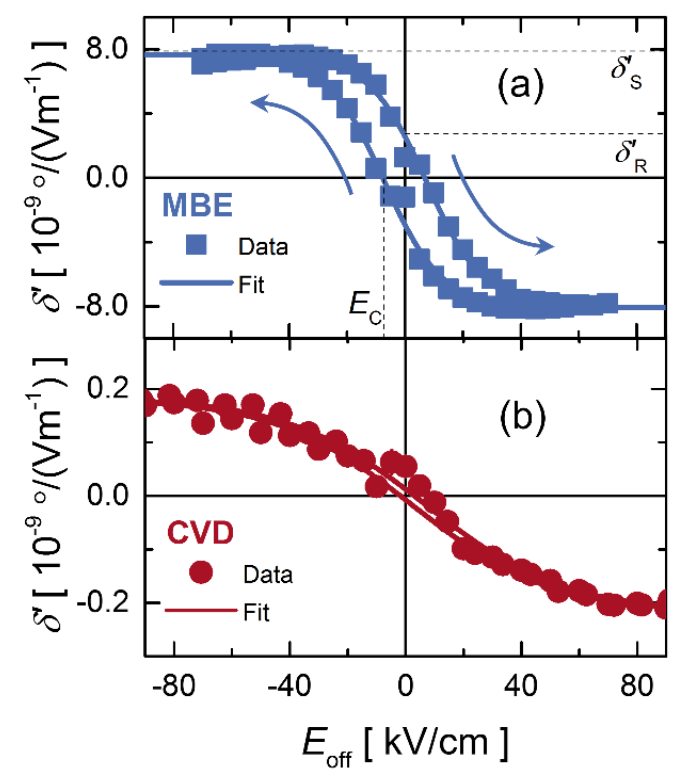

Figure 7.5: (a) The field-normalized induced rotation $\delta$ ' measured as a function of a DC offset bias for BTO deposited by MBE and (b) CVD. Sigmoidal fits (solid lines) are utilized to extract saturation and remanent response, ${ }^{\delta^{\prime}}{ }_{s}$ and $\delta_{r}^{\prime}$ respectively, as well as the coercive field $E_{\text {c. }}$ Sweep direction is indicated by arrows, with sigmoidal fits to the data described in Appendix B .

The ferroelectric nature of the films largely varies, ranging from wide hysteresis and large remanence ratio in MBE-BTO to slim hysteresis and reduced remanence in CVD-BTO. Materials with finite remanence can be used for bistable optical switching, [241] while non-memory materials can be used over a broad continuous tuning range. [242] Due to the low leakage current in the $\mu \mathrm{A}$ range even at high voltages of 35V 
(Appendix B, Table B.1), the tuning applications can be extremely energy efficient. [63] In principle, the variety of properties render the films applicable for a broad range of application, such as low dielectric loss capacitors or ferroelectric storage. [243]

\subsection{DISCUSSION}
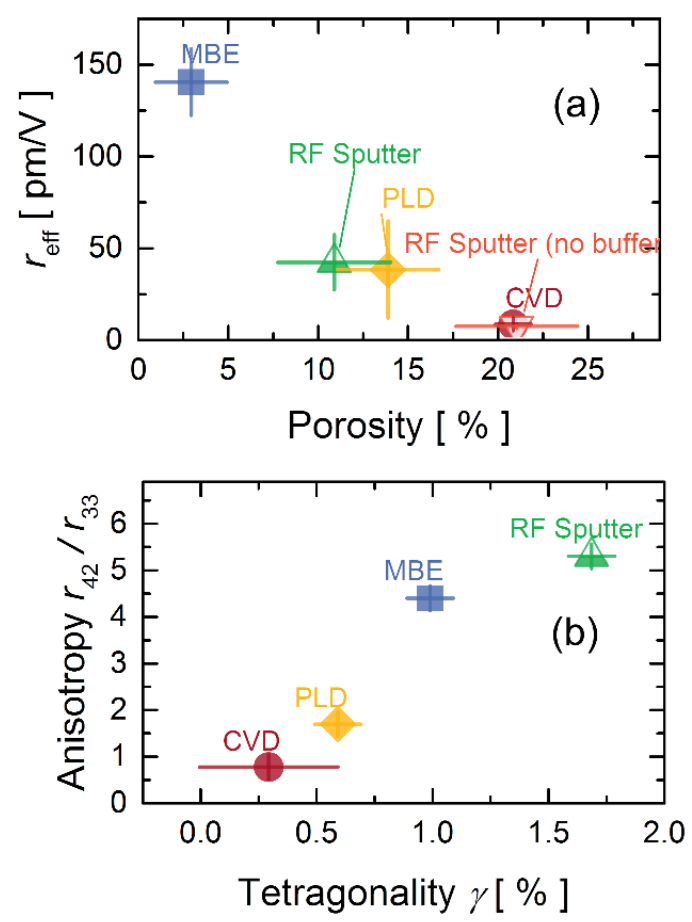

Figure 7.6: (a) Effective Pockels coefficient $r_{\text {eff }}$ vs porosity, (b) anisotropy ratio of Pockels tensor elements $r_{42} / r_{33}$ vs tetragonal distortion $\gamma$, where cubic BTO corresponds to $\gamma=0$, and (c) remanence ratio or squareness factor $\delta_{r}^{\prime} / \delta^{\prime}{ }_{s}$ vs. grain size $e d r$. Open symbols: polycrystalline, filled symbols: textured/epitaxial, halffilled indicates a mixture.

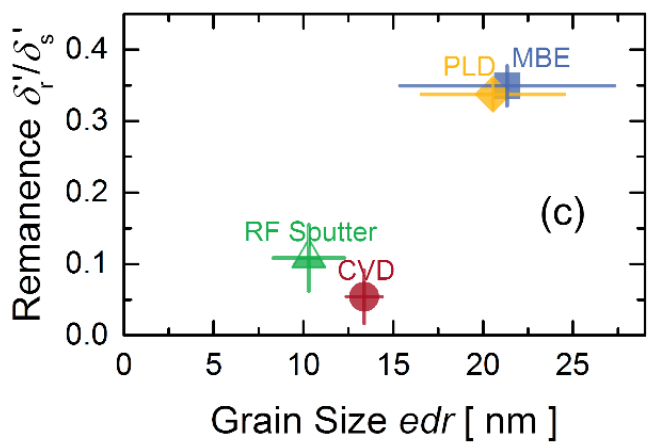

Our results show a strong correlation between the structural and electro-optic properties in BTO thin films. In particular, the consistent reduction of the effective Pockels coefficients for increasing porosity (Figure 7.6(a)) identifies the density of BTO as a key parameter for obtaining a large electrooptic response. In the following, we discuss the physical origin of this correlation. 


\subsubsection{Electric Field Distribution}

First, we consider the impact of porosity on the electric field distribution in the BTO film. In our derivation of the electro-optic response as a function of the Pockels coefficient (Eq.(7.2)) we assume the voltage to drop homogenously along the BTO layer. However, due to the low permittivity of the voids compared to the BTO matrix ( $\varepsilon_{\text {Bто }}>>$ $\varepsilon_{\text {air }}=1$ ), the electric field distribution might be significantly influenced in porous films. Since we cannot perform capacitance-voltage profiling on the exact samples in this study because of the highly-resistive silicon substrate, we assume the permittivity $\varepsilon_{\mathrm{B} T \mathrm{O}}$ to range from 50 to 660 as reported for thin BTO films on Si. [80,234] A reduction of the effective electric field within the BTO matrix for larger porosities would indeed result in a strong reduction of the electro-optic response for porous films even though the Pockels coefficient of the matrix might not have changed.

From this point of view, the values of the Pockels coefficients estimated earlier (Table 7.3) have to be considered as the average Pockels coefficients of the porous films, rather than the actual Pockels coefficient of the BTO crystal. Because the electric field is in the denominator of Eq. (7.2), a porosity correction factor $c_{\mathrm{P}}=E / E_{\mathrm{BTO}}$, defined as the ratio between the electric field in a pore-free BTO crystal $(E)$ and the average electric field in the actual matrix of the porous BTO layer $\left(E_{\mathrm{BTO}}\right)$, can be used to provide a sense of how the Pockels coefficients would scale with porosity.

An electro-static finite elements study has been carried out for different pore geometries. Cylindrically shaped tubes through the material represent the geometry closest to the pores seen in Figure 7.2. For this geometry, even when assuming the largest experimentally observed porosity (20\%) and relative permittivity of $\varepsilon_{\mathrm{B} \text { во }}=660$, the correction factor remains rather moderate $\left(c_{\mathrm{P}} \sim 1.15\right)$ (Appendix B, Figure B.6). In contrast to circular pores, fissures in the material would lead to a correction factor 
between one and two orders of magnitude higher for the same porosity (Appendix B, Figure B.6). While this analysis shows the importance of obtaining crack-free layers for electro-optic applications, on the basis of STEM and AFM analysis (Figure 7.2 and Appendix B, Figure B.2) we preclude fissures as the origin of the reduction of the Pockels effect in our films. The reduction of the electric field in the BTO matrix can thus not be the main origin for the experimentally observed large differences of the electrooptic response between the layers $\left(r_{\mathrm{eff}, \max } / r_{\mathrm{eff} \text {,min }} \sim 20\right)$. This suggests that the Pockels effect of the BTO matrix has to significantly vary among the films in order to account for our observations of a strongly varying electro-optic response.

\subsubsection{Defects in BTO}

Next, we consider factors which could impact the electro-optic response of BTO itself. Because the linear electro-optic response originates from the anharmonic nature of the Ti-O bond and the loss of the inversion symmetry in the tetragonal phase (phonon condensation), defects which affect phonons (breaking the periodicity of the lattice, eg. finite size) [244] or restore centrosymmetric structure (eg. antiphase boundaries) [236,245] are expected to diminish the electro-optic response. This is consistent with Figure 7.6(a), considering pores as defects in the crystal.

In addition to the change in magnitude, it has been already reported that defects such as pores and grain boundaries can stabilize the cubic centrosymmetric phase of BTO, which shows no Pockels effect. [236,246] The reduction of the Pockels effect when increasing the porosity could further be associated to a reduced tetragonality. This hypothesis is verified by comparing the anisotropy of the electro-optic response, defined as the ratio of the Pockels tensor elements $r_{42} / r_{33}$. In bulk single-crystal BTO, this anisotropy ratio is larger than $\sim 10$. [240] We observe in our samples that loss of 
anisotropy correlates with loss of tetragonal distortion $\gamma$ (Figure 7.6(b)). Besides being influenced by porosity, a reduced anisotropy could result also from the influence of strain on the electro-optic tensor. [240]

These two effects, microstructure and symmetry, are in general coupled, as in the case of polycrystalline films containing a higher fraction of cubic BTO. [236] In Figure 7.6(a)-(b), the reduction in the magnitude and anisotropy of the Pockels tensor for cubic, porous, and polycrystalline films indicates a strong dependence of the electro-optic response on crystalline quality. In particular, high tetragonality and minimal porosity in BTO thin films is essential to achieve bulk-like electro-optic performance.

\subsubsection{Memory}

Although all films investigated in our study show ferroelectric functionality, strong variations in the remanent polarization response are observed (Figure 7.5). For the in-plane electrode geometry, low leakage current is needed to prevent film depolarization. [247] In previous studies, functional properties of BTO such as the spontaneous polarization [245] and second harmonic generation [248] improve significantly after poling in fields $\sim 300 \mathrm{kV} / \mathrm{cm}$. [245] Similarly, $\delta$ ' generally reaches a finite remanence value ${ }^{\delta^{\prime}}{ }_{r}$ after poling in sufficiently large electric field. [67] In contrast, $\delta$ ' exists only in the presence of an electric field for materials such as relaxors, where the behavior depends on polar nano-regions. [242] In our study, remanence correlates with grain size edr (Figure 7.6(c)), consistent with previous observations of reduced remanence for small-grained ceramics. [246] The physical origin of this reduced remanence can be structural defects in the films, for example present at grain boundaries, which pin ferroelectric domains. [223,239,247,249,250] The strong variations of the remanence show an additional degree of freedom in engineering the electro-optic 
properties of BTO thin films: While films tuned for large grain sizes could be used for non-volatile memories, [241] small-grained layers might be beneficial for devices requiring periodic poling [242] or filtering. [251]

In summary, we have identified several key correlations between the structural and electro-optic properties of BTO thin films on silicon. The reduction of the effective Pockels coefficients, anisotropy, and remanence in BTO films on Si are concluded to result from crystalline defects and distortions originating from pores in the film, loss of tetragonality, and the finite size of BTO grains. While a linear electro-optic response was measured even for porous polycrystalline BTO, the response is higher by one order of magnitude for dense, tetragonal, epitaxial BTO films.

\subsection{CONCLuSion}

In conclusion, we analyzed BTO thin films of thickness ranging from 70 to 100 $\mathrm{nm}$ deposited by several different methods (MBE, PLD, RF sputtering, and CVD) on STO-buffered $\mathrm{Si}(001)$ to produce an array of samples with varied morphology and structural quality. The effective Pockels coefficient varied from 6 to $140 \mathrm{pm} / \mathrm{V}$ and remanence ratio from 0.35 to 0.04 under this structural variation. Indeed, even highly porous, polycrystalline BTO films were found to have a linear electro-optic response. Our study provides a link between structural and electro-optic properties of BTO thin films, and demonstrates clearly the effectiveness of applying materials science to tailor customized properties of oxide thin films. While obtaining dense and highly tetragonal films is critical to achieve large Pockels coefficient as for example needed in high-speed $\mathrm{BTO} / \mathrm{Si}$ photonic modulators, fabricating layers with large grain sizes is the crucial parameter to maximize the memory window as needed for non-volatile optical storage. For further development of BTO-based Si photonic devices, additional practical aspects 
such as the Curie temperature shift [227,246] should be addressed in future studies, as well as exploring routes including strain engineering and doping to enhance the electrooptic coefficients.

This work was supported by the National Science Foundation (IRES-1358111), the Air Force Office of Scientific Research (FA9550-12-10494 and FA9550-14-1-0090), the European Commission (FP7-ICT-2013-11-619456-SITOGA, H2020-ICT-2015-25688579 PHRESCO), and the SNSF R'Equip Program (Grant No. 206021-144988). 


\section{Chapter 8: Summary and Future Work}

\subsection{SUMMARY}

\subsubsection{Two-Dimensional Electron Gas at Oxide Interfaces}

The first half of this dissertation presents a major step to obtain novel highmobility channels based on the defect-driven oxide interface 2DEG. Due to the strong gettering of oxygen by films of certain metal oxides, these heterostructures offer new solutions for transistor channels and novel sensors. To exploit these benefits of the defect-driven 2DEG, a full route for the formation such an interface is established, starting from the choice of substrate and thin-film deposition method.

In combining two oxides epitaxially to form the $2 \mathrm{DEG}$, several major issues arise. Lattice mismatch broadly limits the choice of materials. In Chapter 3 I demonstrated heteroepitaxy of spinel $\mathrm{Co}_{3} \mathrm{O}_{4}$ on lattice-matched (less than $0.05 \%$ strain) $\mathrm{MgAl}_{2} \mathrm{O}_{4}$ using plasma-assisted molecular beam epitaxy. [87] Another critical concern for 2DEG formation is the heterostructure band alignment. While no free carriers were found in this heterostructure, the $0.75 \mathrm{eV}$ band gap of the cobaltate films is potentially well-situated for catalysis. Finally, complex oxides are often employed for their diverse array of functionalities not found in traditional semiconductors. The first SQUID measurements of these unstrained cobaltate films on $\mathrm{MgAl}_{2} \mathrm{O}_{4}$ indicated a paramagnet-toantiferromagnet transition below $49 \mathrm{~K}$, a $9 \mathrm{~K}$ enhancement compared to the bulk value.

In Chapter 4, I reported on the interfacial conducting layer formed at the surface of STO by depositing an oxide containing $\mathrm{Al}$, a metal from the lower right quadrant of Figure 8.1 (moderate work function with high oxygen affinity) that additionally has a very low diffusivity for oxygen. This result was the first report of MBE-grown epitaxial $\gamma$-alumina films on STO. Crystallinity to reduce lattice mismatch. The large band offset 
measured for this heterostructure offers a strong confining potential for carriers at the interface. This heterostructure serves as a model system to demonstrate a robust defectdriven approach to oxide 2DEG formation. The key parameters identified in Figure 8.1 are a prerequisite for formation of the defect-driven oxide 2DEG and are expected to be quite general design rules. [184]

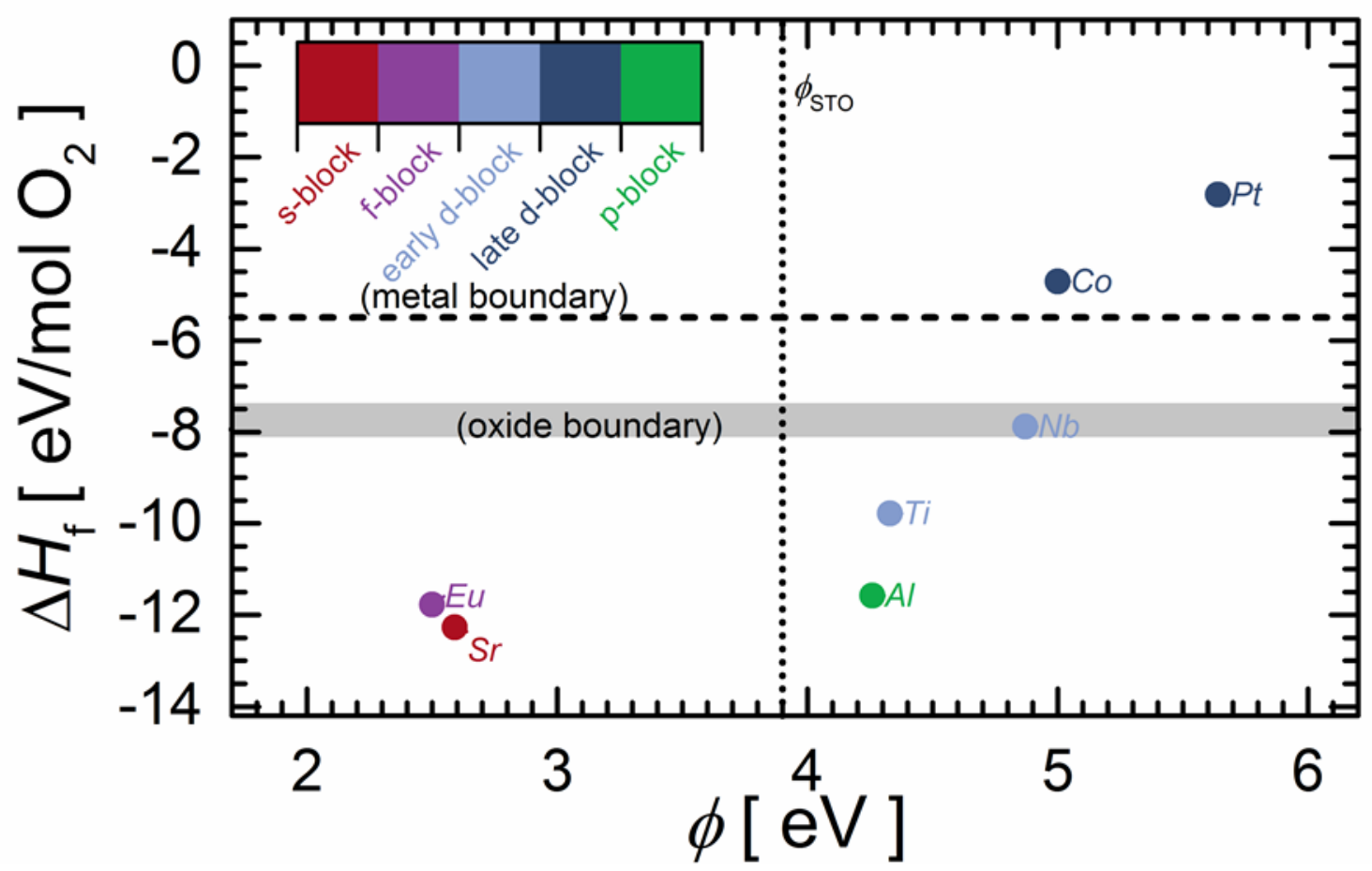

Figure 8.1: Plot of heat of formation of most stable oxide vs. work function for common metallic elements used in this dissertation and in Ref. [184]. The elements naturally group into three categories as subdivided by the solid lines creating three occupied quadrants in the plot. The solid boundaries are based on several empirical reports of oxidation of metals on $\mathrm{SrTiO}_{3}$ and very roughly corresponds to the formation energy of an oxygen vacancy in $\mathrm{SrTiO}_{3}$ and the electron affinity (work function) of n-type $\mathrm{TiO}_{2}$-terminated $\mathrm{SrTiO}_{3}$.

In Chapter 5, this approach is leveraged to explore proximity-induced emergent phenomena by depositing of Eu metal onto $\mathrm{SrTiO}_{3}$ (001). This deposition method enabled crystallization of stoichiometric highly-spin-polarized semiconductor $\mathrm{EuO}$ in 
close proximity to a highly conductive interfacial layer of oxygen-deficient $\mathrm{SrTiO}_{3-\delta}$. Combining these results with first-principles calculations, we uncovered the role of the spin-polarized oxygen vacancy state as the origin of the linear positive MR, suggesting a path towards developing novel nano-oxide spintronic devices based on strong proximity effects. Until now, no other concept using epitaxial EuO to explore proximity effects in the 2DEG has been demonstrated.

\subsubsection{Films of Integrated Nonlinear Oxides}

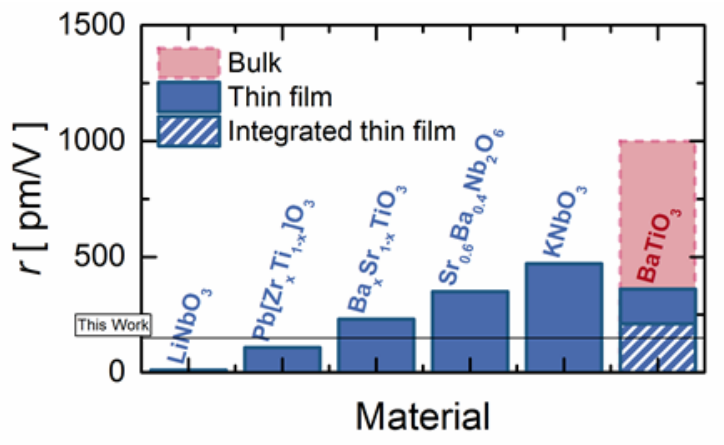

Figure 8.2:Pockels coefficients for oxide thin films.

The large effective Pockels coefficient for high-quality epitaxial BTO films on $\mathrm{Si}$ distinguishes $\mathrm{BaTiO}_{3}$ as a highly promising material for integrated

silicon nanophotonics. However, the linear electro-optic effect in $\mathrm{BaTiO}_{3}$ thin films determined in previous experiments clearly showed deteriorated properties compared to bulk BTO crystals. The goal of the second half of this dissertation was to compare films of widely varied morphology to establish the parameter space for a future manufacturing process. First, in Chapter 6, I studied $\mathrm{BaTiO}_{3}$ films of varied thickness to quantify the Pockels coefficient with respect to crystalline orientation. As a next step, in Chapter 7, I reported on the strong dependence of the Pockels effect in $\mathrm{BaTiO}_{3}$ thin films on their microstructure, and provide guidelines on how to engineer thin films with strong electrooptic response. The $25 \times$ enhancement of the Pockels coefficient indicates a promising route to increase the performance of nonlinear oxides in the two-dimensional limit for the development of novel hybrid silicon photonic platform. By identifying the key structural 
predictors of electro-optic response in $\mathrm{BTO} / \mathrm{Si}$, I outlined the path to leverage the linear electro-optic effect in nanoscale oxides integrated on Si.

\subsection{RECOMMENDATIONS FOR FUTURE WORK}

The relative ease with which STO gives up some of its oxygen is well-known but has The relative ease with which STO gives up some of its oxygen is well-known but has not always been given careful consideration by researchers in oxide thin film growth. [184] This dissertation indicates that novel interfacial phenomena observed at interfaces between STO and another metal oxide should be regarded with more care as STO is likely to become somewhat reduced even under relatively high oxygen pressure

This reducibility of STO, however, can be useful in some cases. In this dissertation, I presented high mobility 2DEGs that have the same properties as the wellknown crystalline LAO-STO interface using a much easier process. One can also use this effect to grow certain metal oxides epitaxially on STO without oxygen such as $\mathrm{SrO}$, EuO and even complex oxides like $\mathrm{LaTiO}_{3}$. This redox reactivity of STO could potentially be exploited to explore other phenomena where the oxygen vacancies themselves interact with the electrons and ions of the metal overlayer.

Even highly porous, polycrystalline BTO films were found to have a linear electro-optic response. My studies provide a link between structural and electro-optic properties of BTO thin films, and demonstrates clearly the effectiveness of applying materials science to tailor customized properties of oxide thin films. While obtaining dense and highly tetragonal films is critical to achieve large Pockels coefficient as for example needed in high-speed BTO/Si photonic modulators, fabricating layers with large grain sizes is the crucial parameter to maximize the memory window as needed for nonvolatile optical storage. For further development of BTO-based Si photonic devices 
(Figure 8.3), additional practical aspects such as the Curie temperature shift should be addressed in future studies, as well as exploring routes including strain engineering and doping to enhance the electro-optic coefficients.

(a)
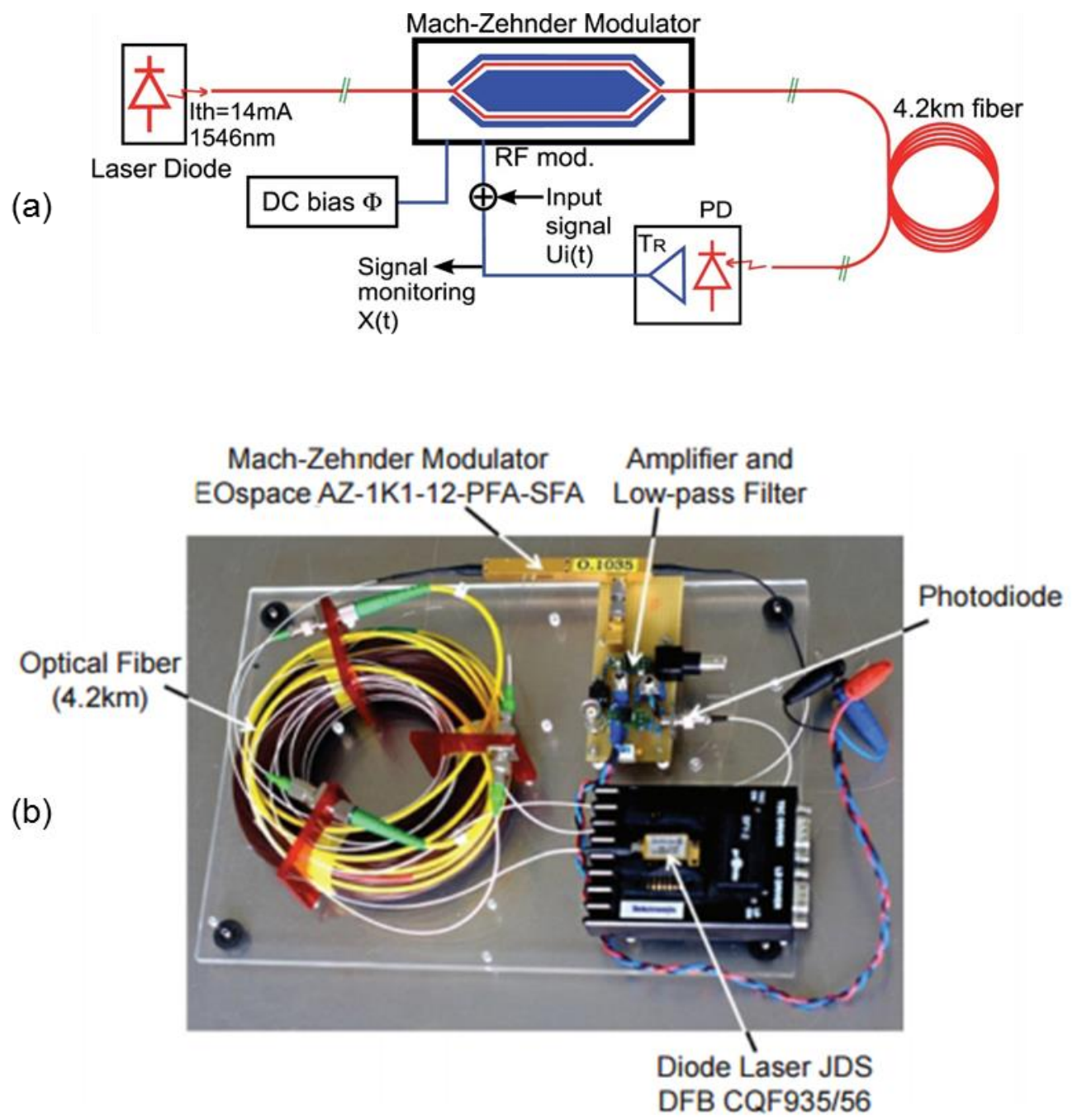

Figure 8.3: (a) Schematic from Ref. [252] and (b) experimental realization of reservoir computing using Mach-Zehnder interferometer on the kilometer scale. [253] 


\section{Appendices}

\section{APPENDiX A: SUPPlement to ChAPTER 4:POSITIVE LiNEAR MAGNETORESISTANCE IN THE TWO-DIMENSIONAL ELECTRON GAS AT THE EUO/SRTIO3 INTERFACE}

\section{Notes}

\section{Note 1: Valence Band Offsets}

Figure A.6(a) displays XPS valence band spectra for the bare STO substrate (blue shading) and 10-nm-thick EuO film (red shading). Band offsets were calculated by both core-level [87] and valence band [133,134] spectroscopy. The valence band maximum (EvBM) for each material was calculated using the linear extrapolation method. [135] By comparing energy offsets between the $\mathrm{Co} 2 p, \mathrm{Al} 2 p$, and valence band edge positions (as summarized in Table 1) using

$V B O_{C L}=\left(E_{E u 3 d}-E_{V B M}\right)_{E u O}-\left(E_{T i 2 p}-E_{V B M}\right)_{S T O}-\left(E_{E u 3 d}-E_{T i 2 p}\right)_{E u O / S T O}$

we calculate a $\mathrm{VBO}_{\mathrm{CL}}$ of $2.44 \mathrm{eV}$.

The VBO can also be determined directly from the valence band spectra. $[133,134]$ For a heterostructure of $1 \mathrm{~nm} \mathrm{EuO/STO}$ a contribution from the substrate is visible along with an attenuated contribution from the substrate (Figure A.6 (a), black open circles). We simulated a fit (purple solid line) to the heterostructure by scaling and offsetting the pure valence band spectra measured for the STO substrate and thick $\mathrm{EuO}$ film. This method uses an "all at once" fit minimizing $\chi^{2}$ by the LevenbergMarquardt algorithm implemented in Igor Pro software (WaveMetrics, Lake Oswego, OR). The difference between the measured and simulated spectra is also plotted (black line). Using this method, we calculate $\mathrm{VBO}_{\text {sim }}=2.26 \mathrm{eV}$, in good agreement with the offset calculated above by the core levels. The band profile is illustrated in Figure A.6(b). 


\section{Note 2: Positive MR Derivation}

Our calculations follow Onose et al.'s work [198], which is based on the Boltzmann transport theory. Based on our DFT calculations, three types of carriers contribute to conductivity: electrons in the spin-split $d_{x y}$ band (up) and electrons in nonmagnetic $d_{x z / y z}$ bands (both up and down). The change of the Fermi energy $\delta \epsilon_{F}$ to first order of the external field is given by

$\delta \epsilon_{F} \approx \frac{1}{2} g \mu_{B} H \frac{D_{x z / y z-}\left(\varepsilon_{F}\right)-D_{x y+}\left(\varepsilon_{F}\right)-D_{x z / y z+}\left(\varepsilon_{F}\right)}{D_{x z / y z-}\left(\varepsilon_{F}\right)+D_{x y+}\left(\varepsilon_{F}\right)+D_{x z / y z+}\left(\varepsilon_{F}\right)}$,

And the corresponding change of conductivity can be expressed as

$$
\begin{aligned}
& \sigma(H)-\sigma(0) \approx \frac{1}{3} e^{2}\left\{\left[\left(2 \frac{\tau_{x y+}}{m_{x y+}^{*}}-2 \frac{\tau_{x z / y z-}}{m_{x z / y z-}^{*}}\right)+\left(\tau_{x y+} v_{x y+}\left(\varepsilon_{F}\right)^{2} \frac{\partial \ln D_{x y+}\left(\varepsilon_{F}\right)}{\partial\left(\varepsilon_{F}\right)}-\right.\right.\right. \\
& \left.\left.\tau_{x z / y z-} v_{x z / y z-}\left(\varepsilon_{F}\right)^{2} \frac{\partial \ln D_{x z / y z-}\left(\varepsilon_{F}\right)}{\partial\left(\varepsilon_{F}\right)}\right)\right] \frac{D_{x z / y z-}\left(\varepsilon_{F}\right) D_{x y+}\left(\varepsilon_{F}\right)}{D_{x z / y z-}\left(\varepsilon_{F}\right)+D_{x z / y z+}\left(\varepsilon_{F}\right)+D_{x y+}\left(\varepsilon_{F}\right)}+\left[\left(2 \frac{\tau_{x z / y z+}}{m_{x z / y z+}^{*}}-\right.\right. \\
& \left.2 \frac{\tau_{x z / y z-}}{m_{x z / y z-}^{*}}\right)+\left(\tau_{x z / y z+} v_{x z / y z+}\left(\varepsilon_{F}\right)^{2} \frac{\partial \ln D_{x z / y z+}\left(\varepsilon_{F}\right)}{\partial\left(\varepsilon_{F}\right)}-\right. \\
& \left.\left.\left.\tau_{x z / y z-} v_{x z / y z-}\left(\varepsilon_{F}\right)^{2} \frac{\partial \ln D_{x z / y z-}\left(\varepsilon_{F}\right)}{\partial\left(\varepsilon_{F}\right)}\right)\right] \frac{D_{x z / y z-}\left(\varepsilon_{F}\right) D_{x z / y z+}\left(\varepsilon_{F}\right)}{D_{x z / y z-}\left(\varepsilon_{F}\right)+D_{x z / y z^{+}}\left(\varepsilon_{F}\right)+D_{x y+}\left(\varepsilon_{F}\right)}\right\} g \mu_{B} H,
\end{aligned}
$$

Since initially $d_{x z / y z}$ bands are nonmagnetic, contributions from up and down $d_{x z / y z}$ bands should cancel with each other. Only the term in the first bracket survives. By replacing $\sigma$ with $\frac{1}{\rho}$, the change of the resistivity with magnetic field can be expressed as,

$$
\begin{gathered}
\rho(H)-\rho(0) \approx-\frac{1}{3} e^{2}\left\{\left[\left(2 \frac{\tau_{x y+}}{m_{x y+}^{*}}-2 \frac{\tau_{x z / y z-}}{m_{x z / y z-}^{*}}\right)+\right.\right. \\
\left(\tau_{x y+} v_{x y+}\left(\varepsilon_{F}\right)^{2} \frac{\partial \ln D_{x y+}\left(\varepsilon_{F}\right)}{\partial\left(\varepsilon_{F}\right)}-\right. \\
\left.\left.\left.\tau_{x z / y z_{-}} v_{x z / y z_{-}}\left(\varepsilon_{F}\right)^{2} \frac{\partial \ln D_{x z / y z^{-}}\left(\varepsilon_{F}\right)}{\partial\left(\varepsilon_{F}\right)}\right)\right] \frac{D_{x z / y z^{-}}\left(\varepsilon_{F}\right) D_{x y+}\left(\varepsilon_{F}\right)}{D_{x z / y z_{-}}\left(\varepsilon_{F}\right)+D_{x z / y{ }^{+}}\left(\varepsilon_{F}\right)+D_{x y+}\left(\varepsilon_{F}\right)}\right\} \rho(0)^{2} g \mu_{B} H
\end{gathered}
$$

where $\tau, m^{*}, v\left(\epsilon_{F}\right)$ and $D\left(\epsilon_{F}\right)$ are lifetime, effective mass, Fermi velocity and density of states at the Fermi level of corresponding carriers, respectively. For different carriers, $m^{*}, v\left(\epsilon_{F}\right)$ and $D\left(\epsilon_{F}\right)$ are of the same order, while the relaxation time $\tau_{x y}$ at the interface is much shorter due to strong scattering [254-257]. Taking the limit $\tau_{x y} \rightarrow 0$, the change of resistivity can be asymptotic as, 


$$
\begin{aligned}
& \rho(H)-\rho(0) \approx \frac{1}{3} e^{2}\left[\left(2 \frac{\tau_{x z / y z-}}{m_{x z / y z-}^{*}}+\right.\right. \\
& \left.\left.\tau_{x z / y z-} v_{x z / y z_{-}}\left(\varepsilon_{F}\right)^{2} \frac{\partial \ln D_{x z / y z-}\left(\varepsilon_{F}\right)}{\partial\left(\varepsilon_{F}\right)}\right) \frac{D_{x z / y z-}\left(\varepsilon_{F}\right) D_{x y+}\left(\varepsilon_{F}\right)}{D_{x z / y z^{-}}\left(\varepsilon_{F}\right)+D_{x z / y z_{-}-}\left(\varepsilon_{F}\right)+D_{x y+}\left(\varepsilon_{F}\right)}\right] \rho(0)^{2} g \mu_{B} H
\end{aligned}
$$

where the change of $\rho$ to the linear order of $\mathrm{H}$ is consistent with experimental results. Further studies of the carrier lifetime would provide a quantitative estimate of the magnitude of the MR.

\section{Figures}
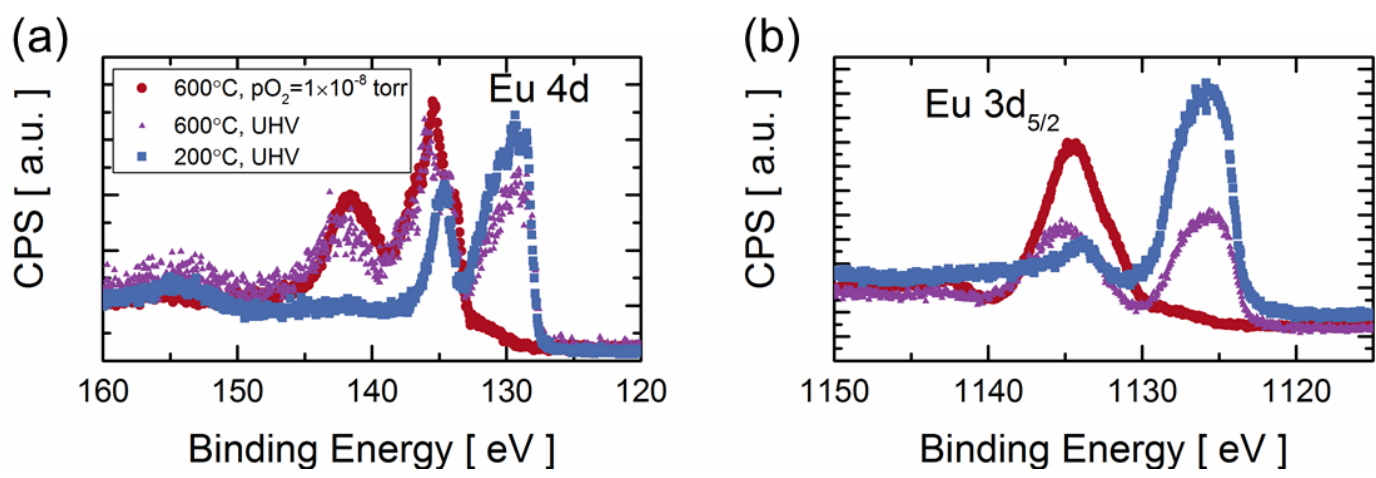

Figure A.1: XPS Eu (a) $4 d$ and (b) $3 d$ core level measurements for varied substrate temperature and oxygen partial pressure. 


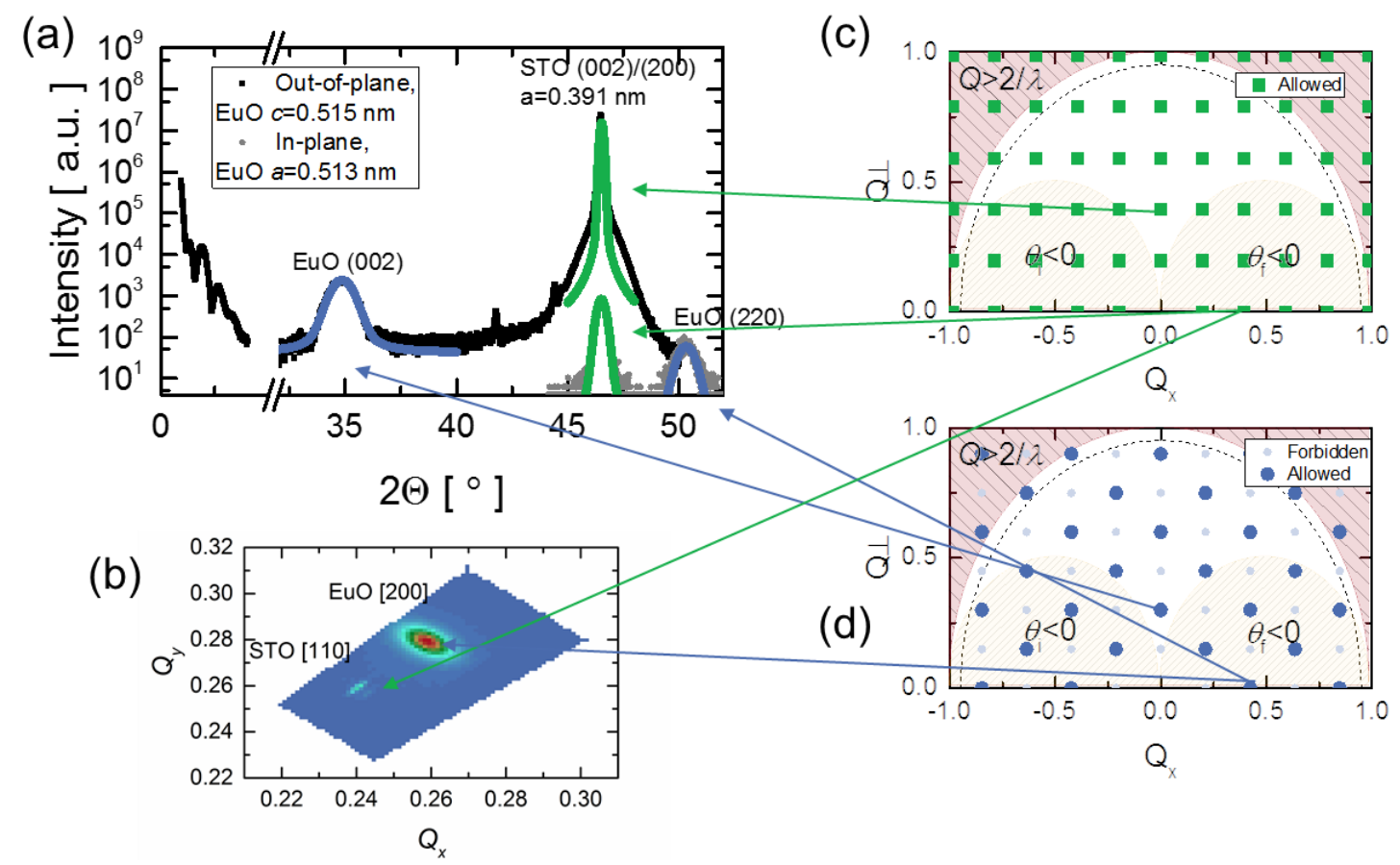

Figure A.2: (a) X-ray diffraction coupled scans with corresponding (b) in-plane reciprocal space map and positions in reciprocal space for (c) STO and (b) $\mathrm{EuO}$ with a $45^{\circ}$ rotation of the surface unit cell. 

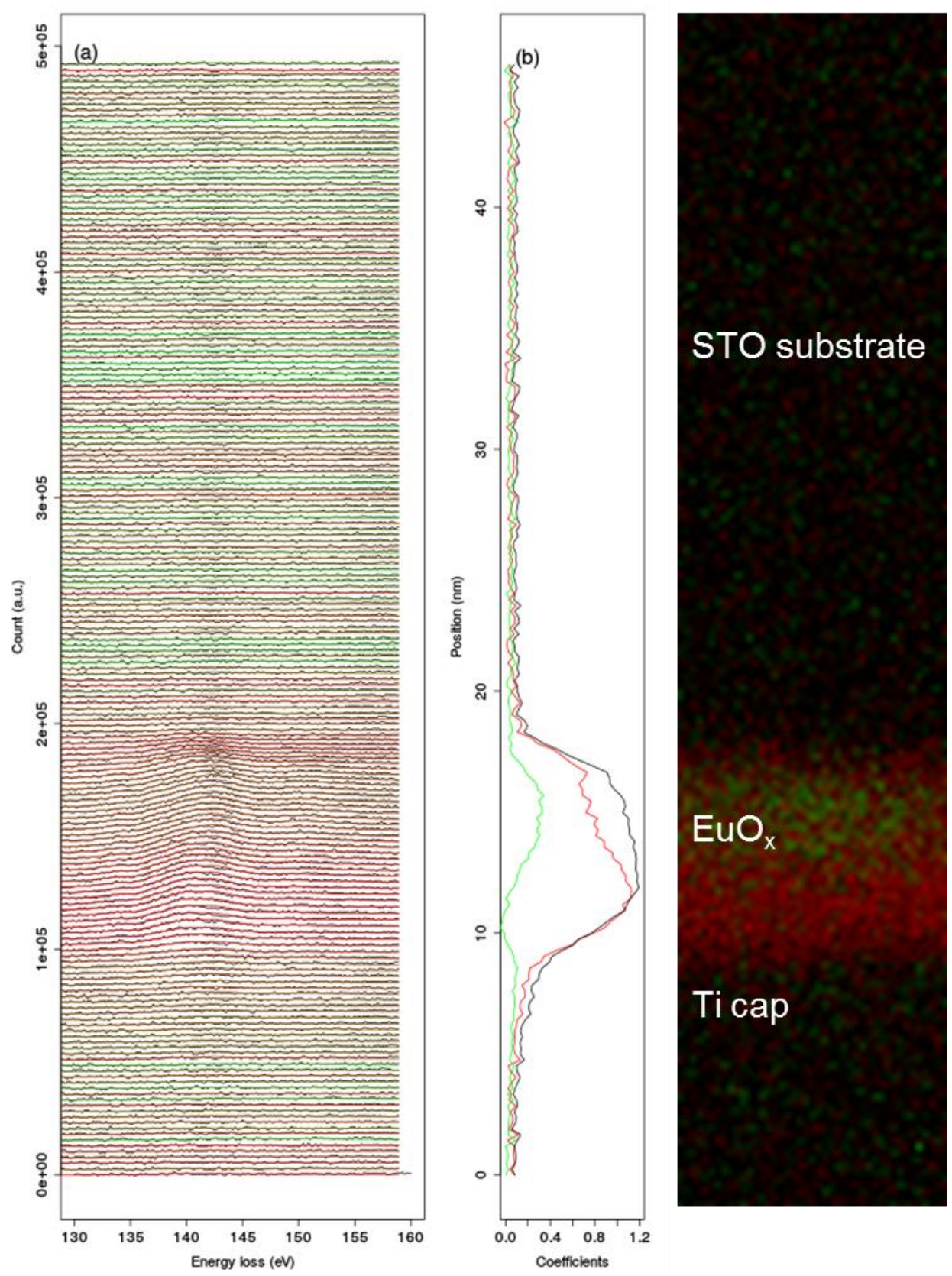

Figure A.3: MLLS fitting. Red is $\mathrm{EuO}$ and $\mathrm{Green}$ is $\mathrm{Eu}_{2} \mathrm{O}_{3}$. Only a little $\mathrm{Eu}^{3+}$ is present in the disordered region. (Reference spectra taken from Mairoser et al. [194]). 
(a)
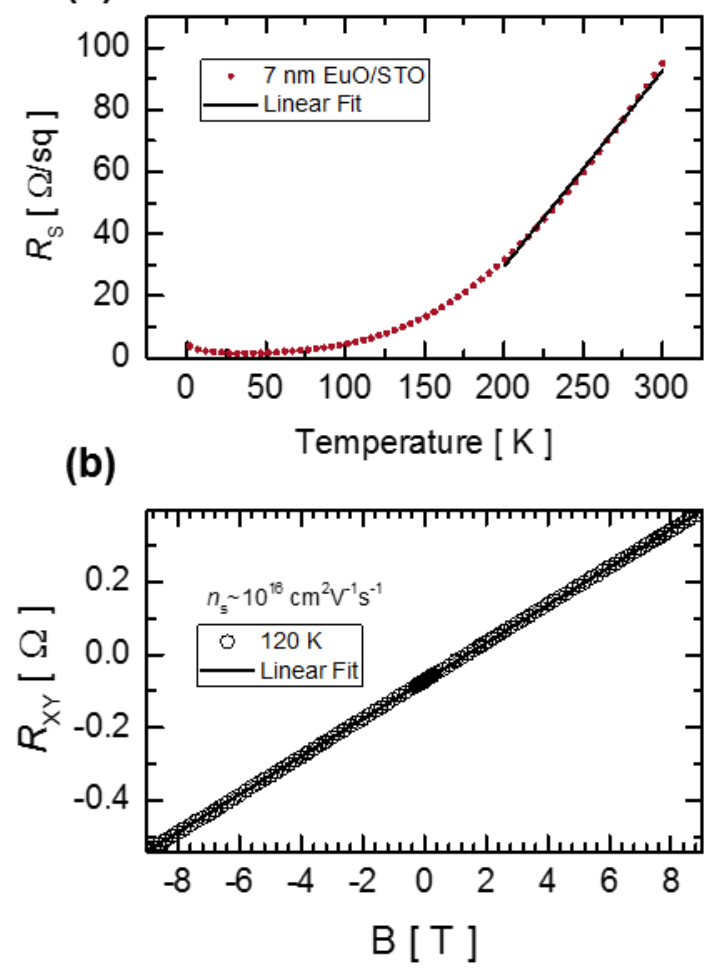

Figure A.4: (a) Sheet resistance for a 7$\mathrm{nm} \mathrm{EuO} \mathrm{film} \mathrm{as} \mathrm{a} \mathrm{function} \mathrm{of}$ temperature. (b) Hall resistance $R_{\mathrm{XY}}$ for a $7-\mathrm{nm} \mathrm{EuO} \mathrm{film} \mathrm{at} 120 \mathrm{~K}$. Solid lines indicate linear fits.

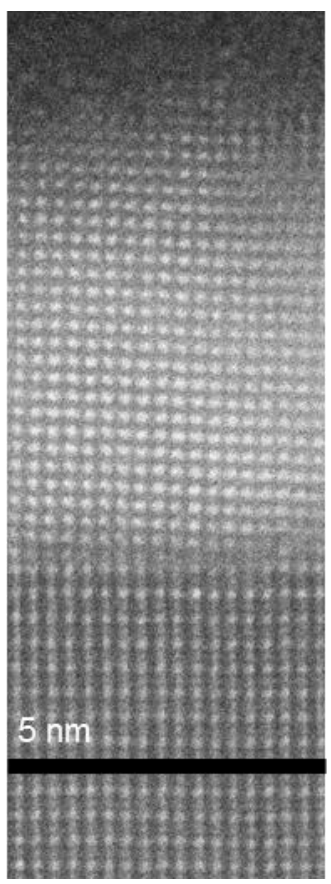

Figure A.5: High-angle annular dark-field scanning transmission electron microscopy [110]-projection image of the EuO/STO interface. 


$$
\text { (a) }
$$

$\frac{1}{3}$
$\frac{0}{0}$
0
0

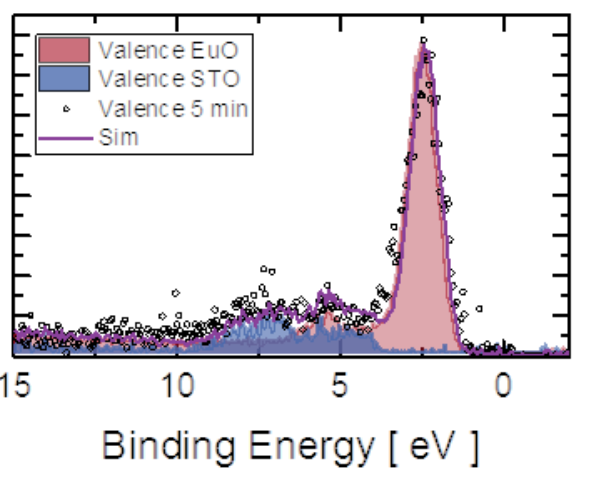

(b)

$E_{c}$

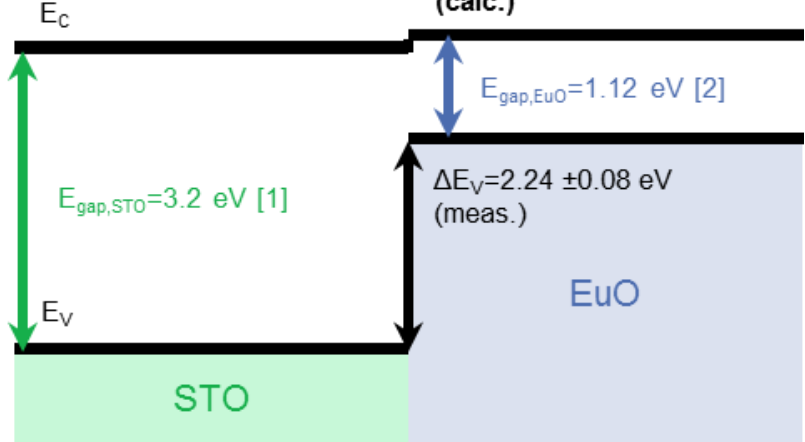

Figure A.6: (a) To determine the valence band offset, the spectra for pure EuO (red shading) and STO (blue shading) were scaled, offset, and added together to create a simulated fit (purple line) to the measured valence band spectra for a heterostructure of 1 $\mathrm{nm}$ EuO on STO (black open circles). (b) Schematic of band alignment. 


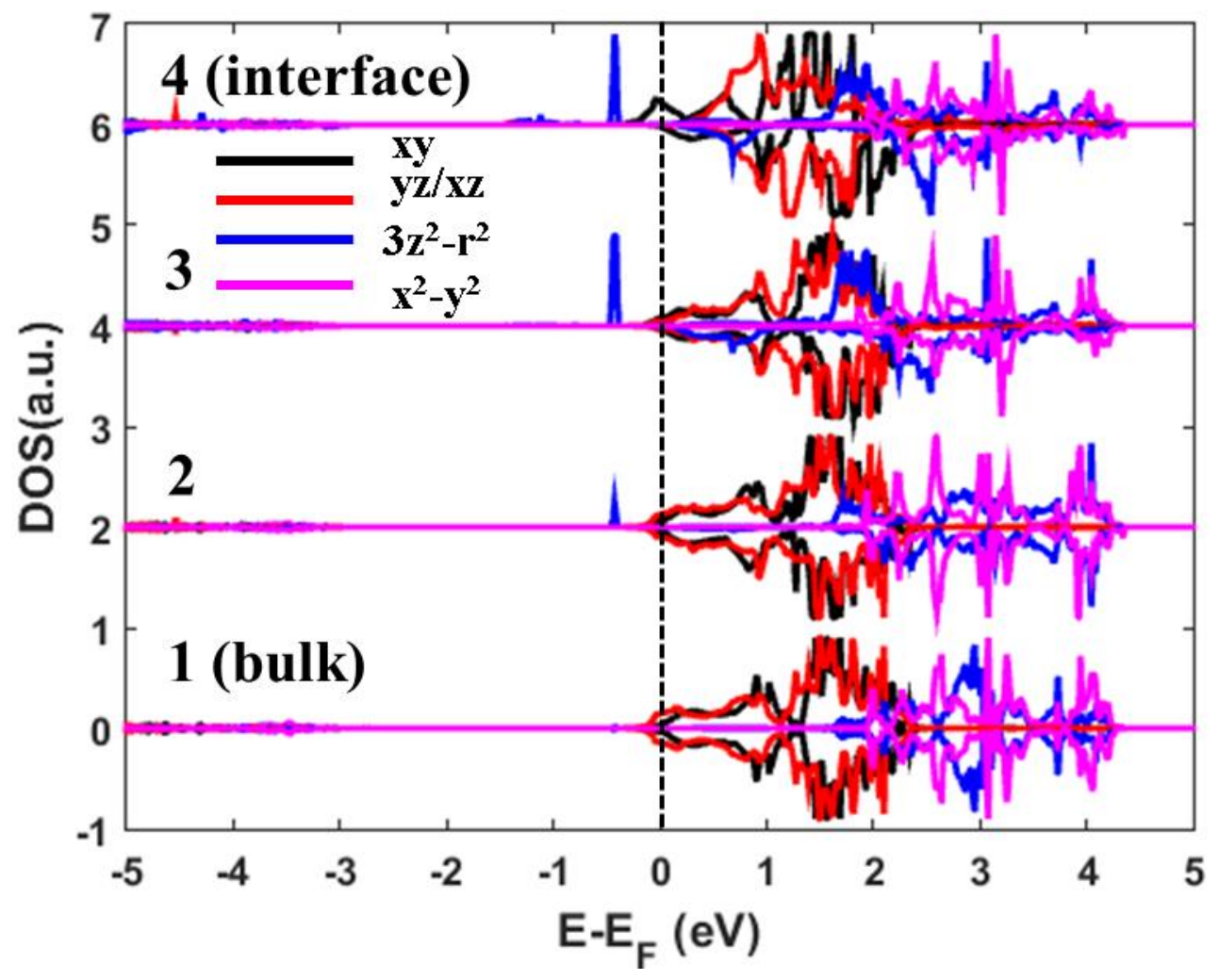

Figure A.7: The partial density of states projected onto $\mathrm{Ti}$ ions at each layer. Interface $\mathrm{TiO}_{2}$ layer is at top and middle bulk $\mathrm{TiO}_{2}$ layer is at bottom. $\mathrm{d}_{\mathrm{xy}}$ orbitals are marked as black line. $\mathrm{d}_{\mathrm{xz}}$ and $\mathrm{d}_{\mathrm{yz}}$ orbitals are marked as red line. $\boldsymbol{d}_{3 \mathrm{z}^{2}-\boldsymbol{r}^{2}}$ orbitals are marked as blue and $\boldsymbol{d}_{\boldsymbol{x}^{2}-\boldsymbol{y}^{2}}$ orbitals are marked as magenta. 


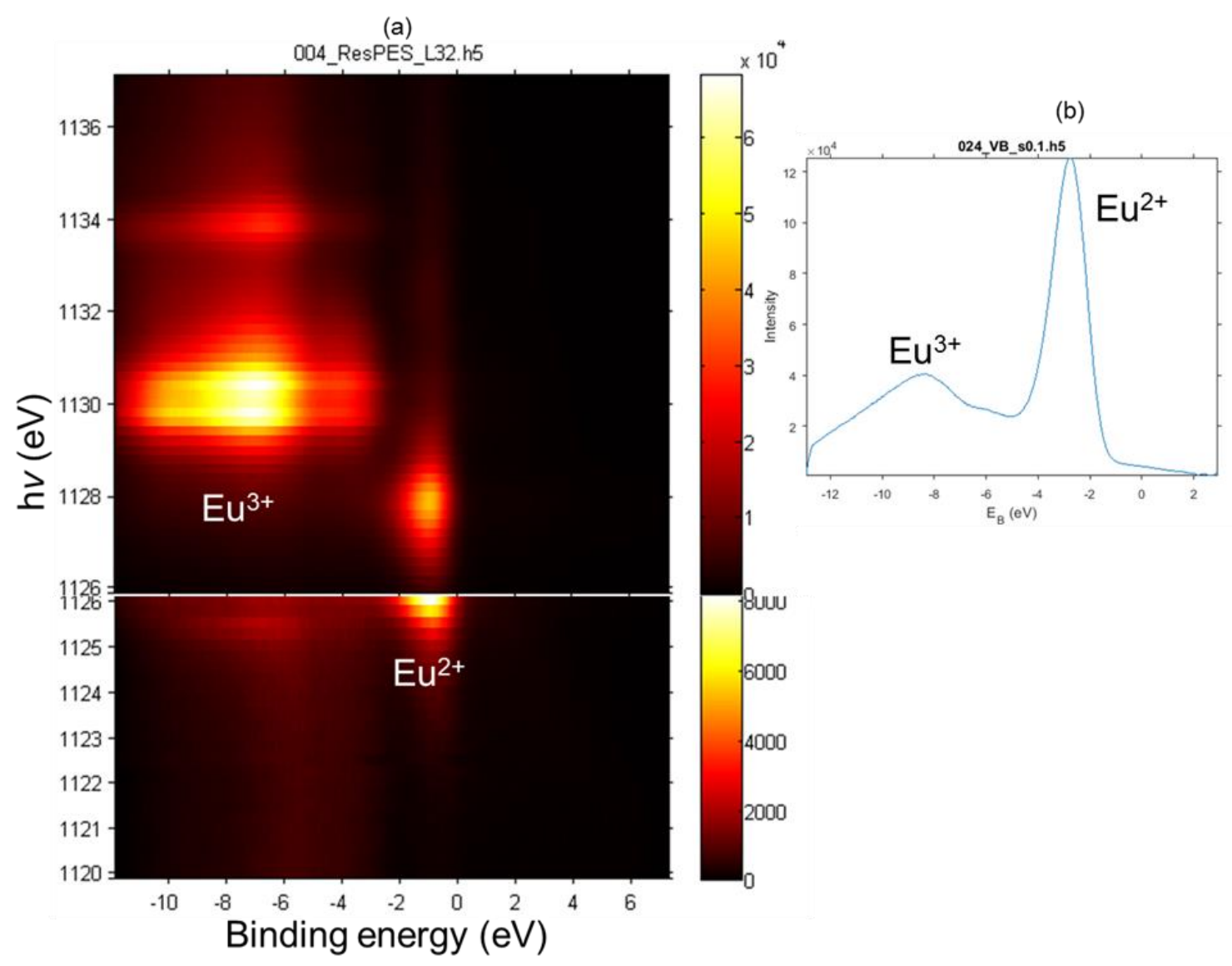

Figure A.8: Resonance photoemission spectra of valence band near Eu 3d threshold. Strong resonance of the $\mathrm{Eu}^{2+}$ states in the valence band at $\mathrm{h} v=1128 \mathrm{eV}$ and $\mathrm{Eu}^{3+}$ states at $\mathrm{hv}=1130 \mathrm{eV}$. No resonating states at the $E_{\mathrm{F}}$. (b) Valence band spectrum at $\mathrm{h} v=457 \mathrm{eV}$ shows the prevalence of $\mathrm{Eu}^{2+}$. 


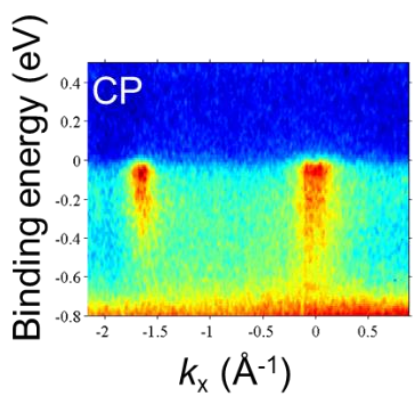

\section{Photoelectron images of 2DES \\ for $\mathrm{hv}=460.3$ and $466 \mathrm{eV}$ \\ Burnt spot of the sample}
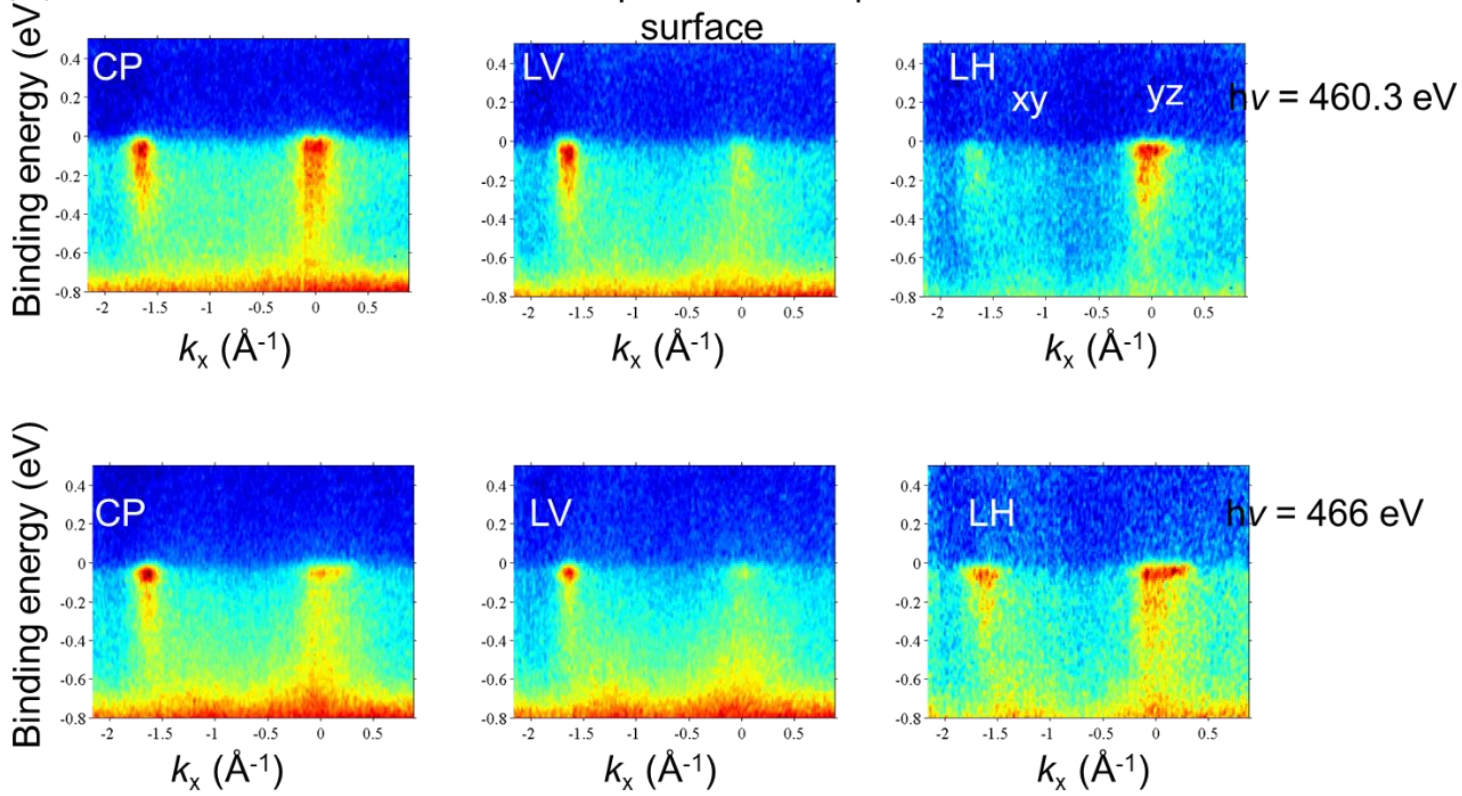

Figure A.9: Photoelectron images of 2DES for hv $=460.3$ and $466 \mathrm{eV}$ for a burnt spot of the sample surface. 


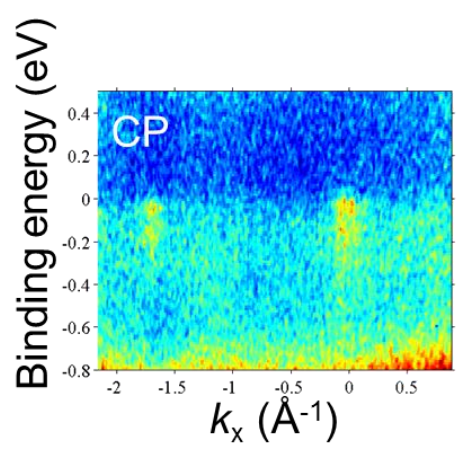

\section{Photoelectron images of 2DES}

for $\mathrm{h} v=460.3$ and $466 \mathrm{eV}$

Fresh spot of the sample
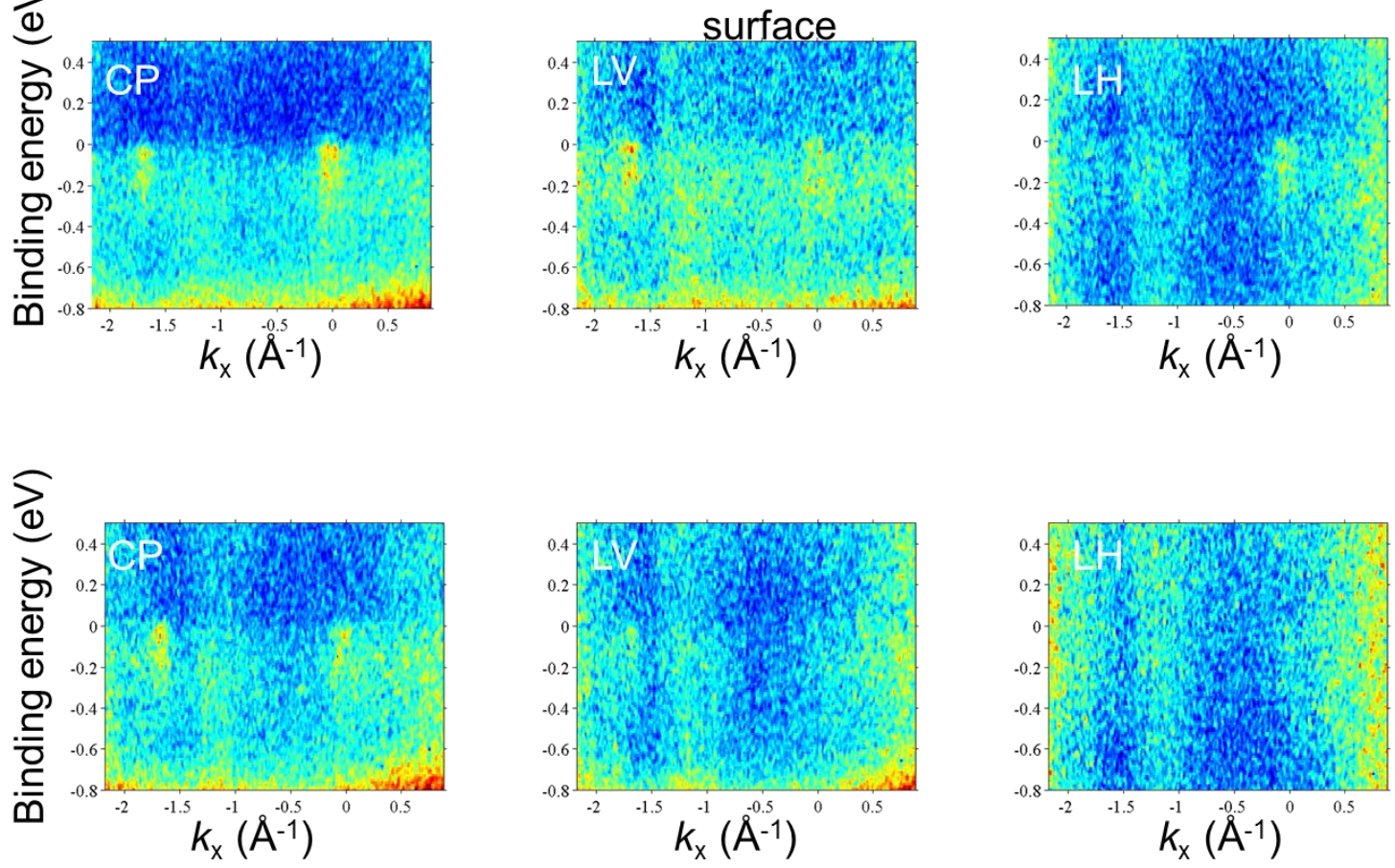

Figure A.10: Photoelectron images of 2DES for $\mathrm{h} v=460.3$ and $466 \mathrm{eV}$ for a fresh spot of the sample surface. 


\section{"0" state}
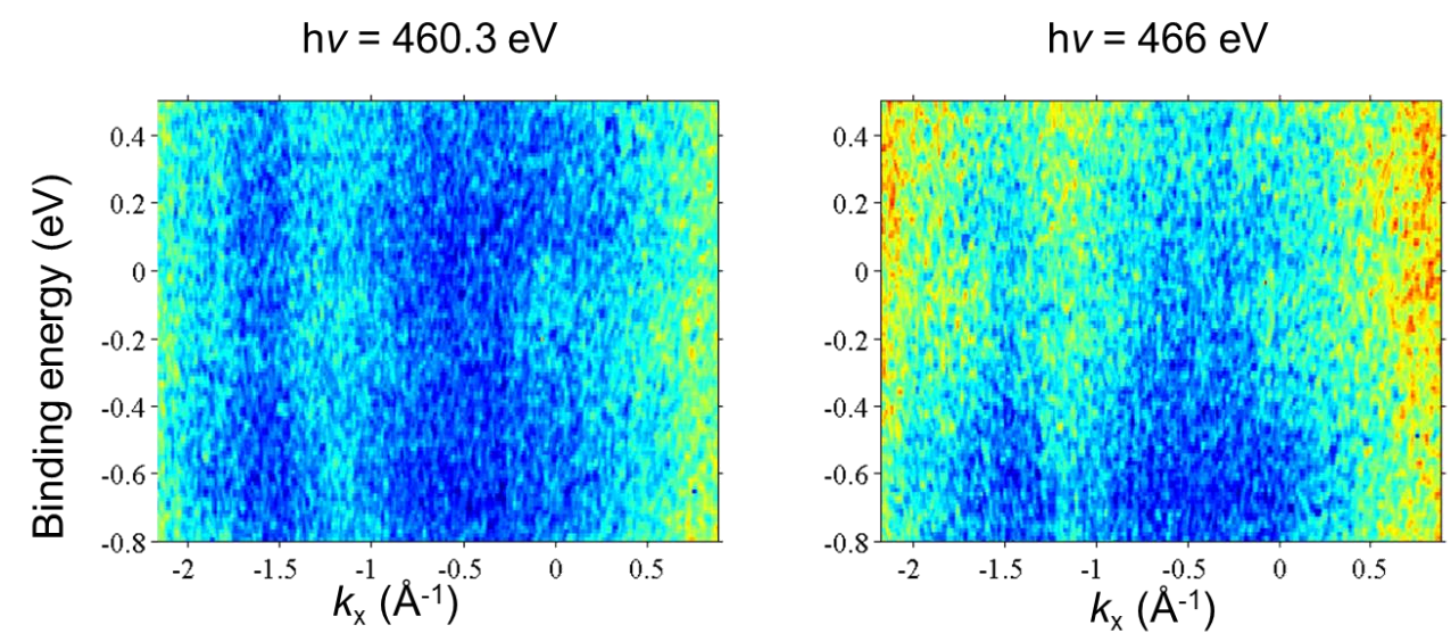

Figure A.11: "0" state. 


\section{APPENDIX B: SUPPLEMENT TO CHAPTER 7:MICROSTRUCTURE AND FERROELECTRICITY OF BATIO3/SI FOR INTEGRATED PHOTONICS}
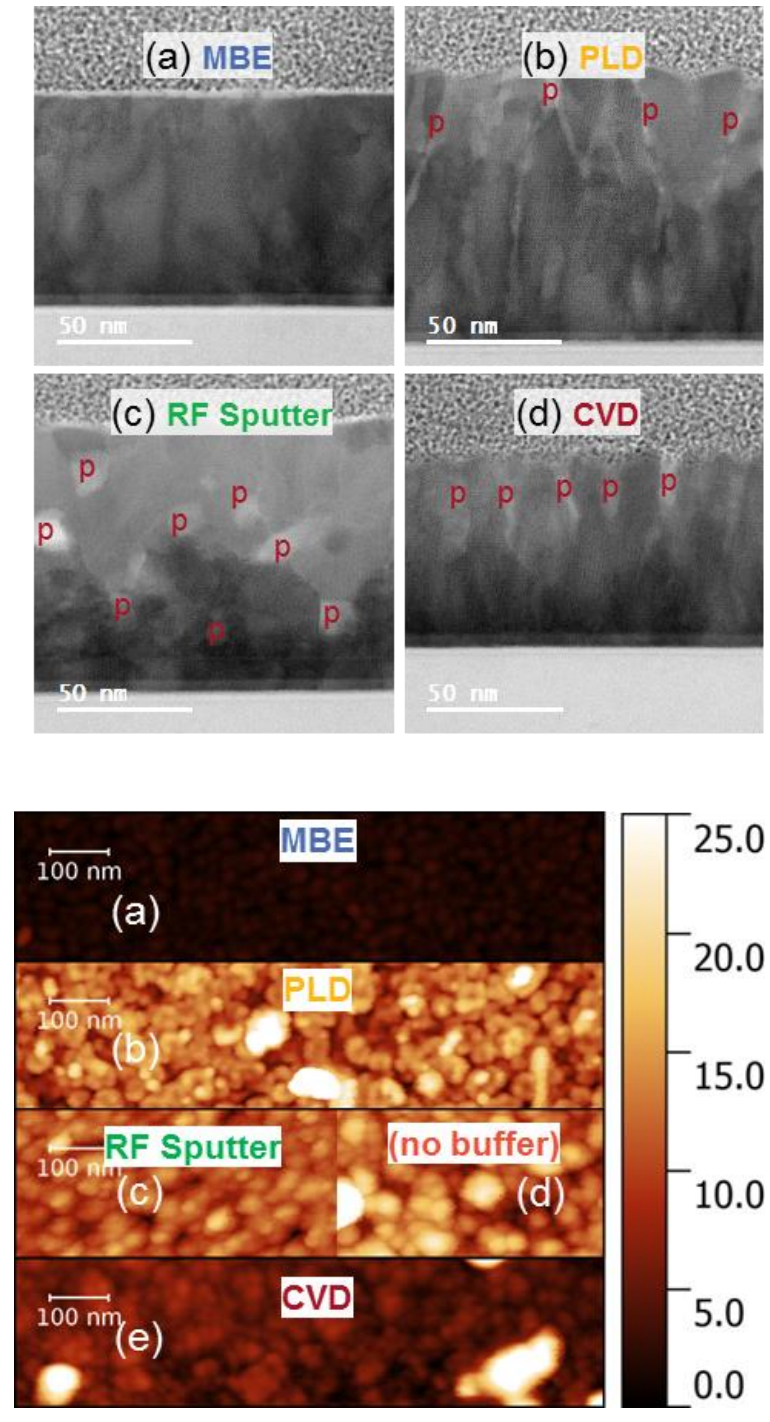

Figure B.1: Bright-field scanning transmission electron microscopy (STEM) images of barium titanate (BTO) on strontium titanate $(\mathrm{STO}) / \mathrm{Si}$ pseudosubstrates deposited by (a) molecular beam epitaxy (MBE), (b) pulsed laser deposition (PLD), (c) RF sputtering, and (d) chemical vapour deposition (CVD). Brighter areas in the film correspond to reduced film density, pores, labeled "p".

Figure B.2: (a) Atomic force microscopy images for BTO deposited by MBE, (b) PLD (c) RF sputtering, (d) RF sputtering (no buffer), and (e) CVD. Root mean square (RMS) surface roughness values are summarized in Table B.1. 
(a)

(b)

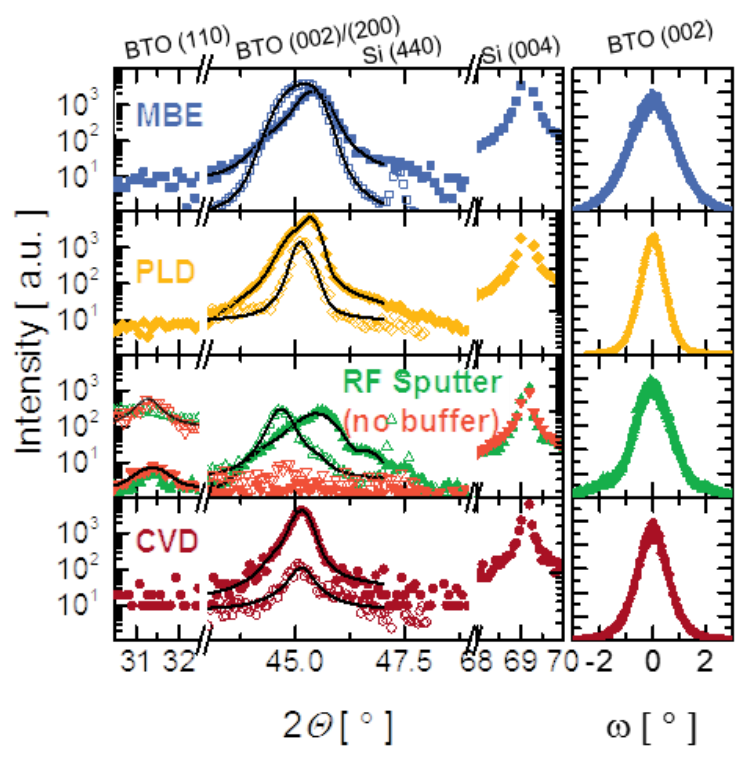

Figure B.3: (a) X-ray diffraction patterns for BTO deposited by MBE, PLD, RF sputtering on STO/Si pseudosubstrate (green up triangles), RF sputtering directly on $\mathrm{Si}$ (orange down triangles), and CVD. Out-of-plane and grazing incidence inplane scans are shown by filled and open symbols, respectively. A sum of three Voigt functions has been fitted to each pair of scans, as shown by solid lines. (b) Corresponding rocking curves of the BTO (002) reflection (not available for BTO sputtered directly on $\mathrm{Si}$ ). The rocking curves full width at half maximum (RC FWHM) are summarized in Table B.1. 
(a)

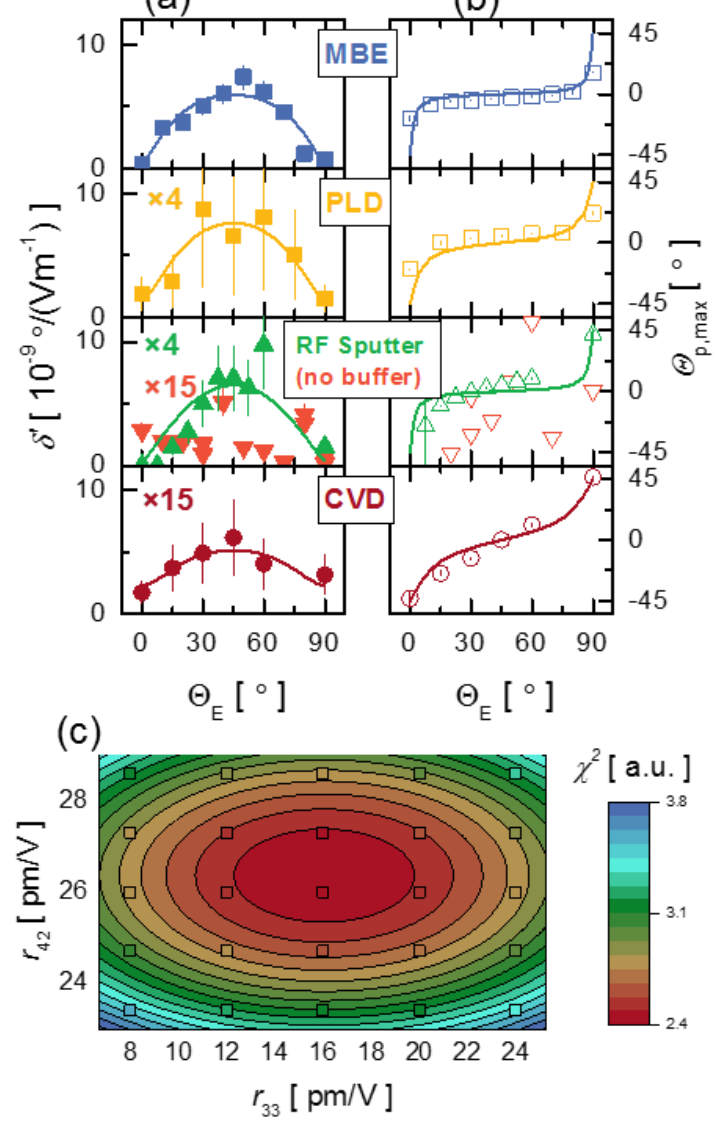

Figure B.4: (a) The field-normalized induced rotation $\delta$ ' was measured (symbols) and simulated (lines) for varied orientation $\theta_{\mathrm{E}}$ of in-plane electric field for BTO films deposited by MBE, PLD, RF sputtering on STO/Si pseudosubstrate (green up triangles), RF sputtering directly on Si (orange down triangles), and CVD.

(b) The orientation of the incident polarization of the linearly polarized light at which the maximum in $\delta$ ' is observed, $\theta_{\mathrm{p}, \max }$, was also measured and simulated. (c) Example of data fitting to select best-fit parameters $r_{42}$ and $r_{33}$. Squares: simulated points, contours: 2-d Gaussian fit.

Figure B.5: (a) The field-normalized

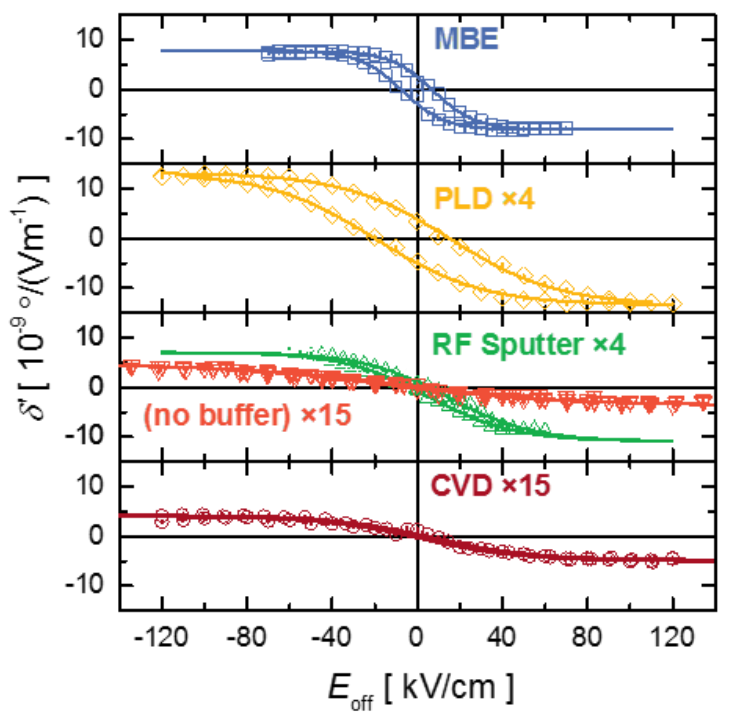
induced rotation $\delta$ ' measured as a function of a DC offset bias for BTO deposited by MBE, PLD, RF sputtering on STO/Si pseudosubstrate (green up triangles), RF sputtering directly on Si (orange down triangles), and CVD. Sigmoidal fits (solid lines) are utilized to extract saturation and remanent response, $\delta_{\mathrm{S}}$ ' and $\delta_{\mathrm{R}}$ ' respectively, as well as the coercive field $E_{\text {c. }}$ 


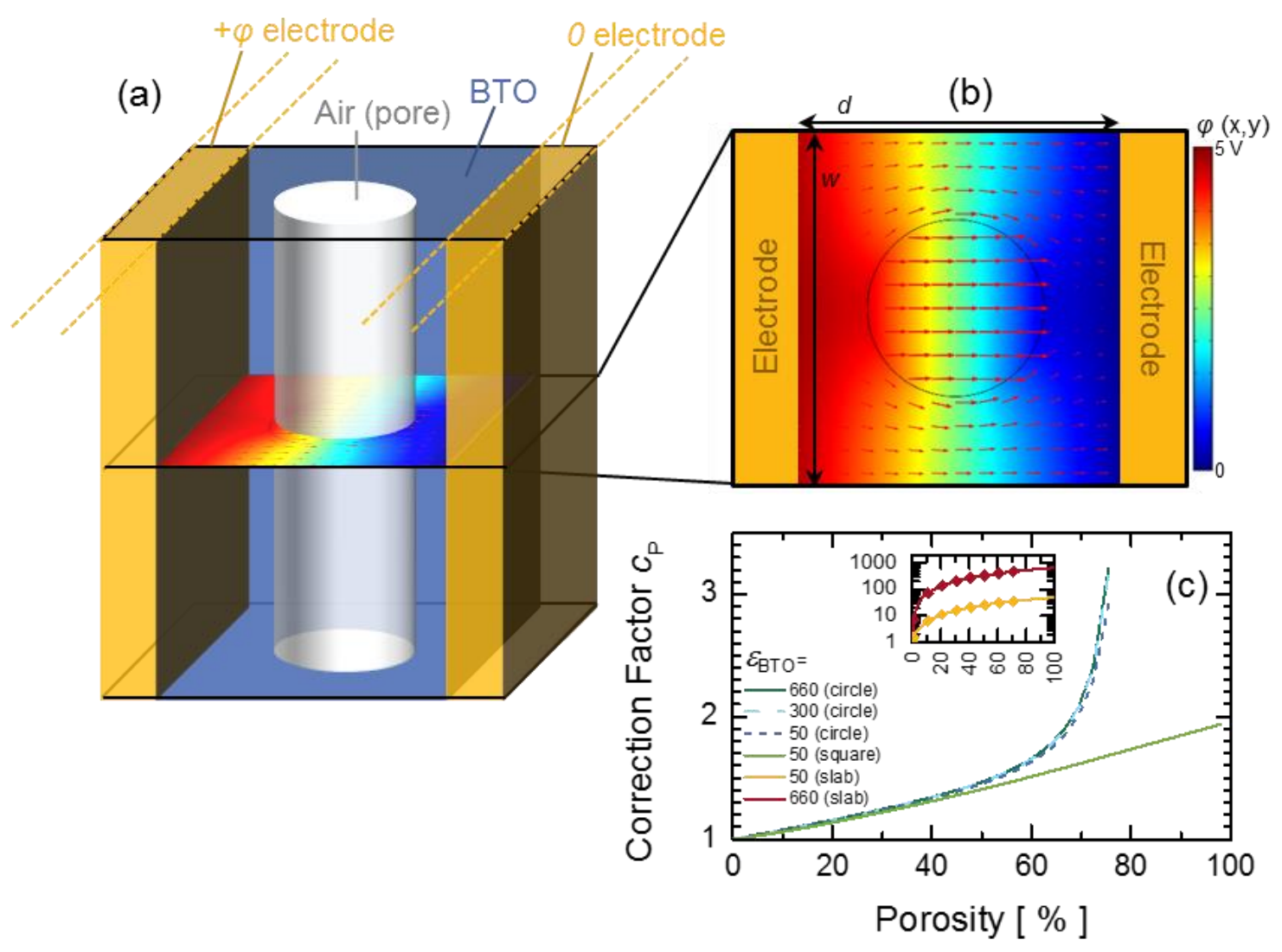

Figure B.6: (a) Schematic of the geometry used for simulating the electric field distribution in porous BTO layers. While a cylindrical pore is shown here, we also considered rectangular cuboid pores. (b) Example of the electric field distribution in a cross-sectional plane as indicated in Fig. (a). The simulations were carried out using the commercial finite-element solver COMSOL Multiphysics. The color scale indicates the electric potential $\varphi$, with arrows indicating magnitude and direction of the corresponding electric field. Simulation parameters and results are summarized in Table B.3(c) Correction factors $c_{\mathrm{P}}$ are calculated for varied porosities $p$, dielectric constants $\varepsilon$ Вто, and pore cross-section shapes (circle, square, or rectangular slab of width $w$ (inset)). 


\begin{tabular}{cccc}
\hline \hline BTO Method & $\begin{array}{c}\text { RMS } \\
\text { Roughness } \\
{[\mathrm{nm}]}\end{array}$ & $\begin{array}{c}\text { RC FWHM } \\
{\left[{ }^{\circ}\right]}\end{array}$ & $\begin{array}{c}\text { Leakage } \\
\text { Current } \\
{[\mathrm{A}]}\end{array}$ \\
\hline MBE & 0.53 & 2.1 & $10^{-5}$ \\
PLD & 3.10 & 1.2 & $10^{-7}$ \\
RF Sputter & 2.40 & 1.7 & $10^{-4}$ \\
CVD & 1.61 & 1.4 & $10^{-6}$ \\
RF Sputter (no buffer) & 3.10 & N/A & $10^{-6}$ \\
\hline \hline
\end{tabular}

Table B.1: Summary of additional film structural and electrical parameters. From the atomic force microscopy images in Figure B.2, the RMS surface roughness can be extracted for all samples. The FWHM of the rocking curves in Figure B.3(b) were also calculated. The leakage current at a DC bias of $35 \mathrm{~V}$ is shown for all samples.

\begin{tabular}{cccccccc}
\hline \hline \multirow{2}{*}{ BTO Method } & $\left.\begin{array}{c}t \\
{[\mathrm{~nm}]}\end{array}\right] \begin{array}{c}E_{\mathrm{AC}} \\
{[\mathrm{kV} / \mathrm{cm}]}\end{array}$ & $\left.\begin{array}{c}E_{\text {off }} \\
{[\mathrm{kV} / \mathrm{cm}]}\end{array}\right]$ & $\begin{array}{c}d x \\
\mathrm{~cm} / \mathrm{kV}]\end{array}$ & $\begin{array}{c}r_{33} * \\
\mathrm{pm} / \mathrm{V}]\end{array}$ & $\begin{array}{c}r_{42} * \\
{[\mathrm{pm} / \mathrm{V}]}\end{array}$ \\
\hline \hline MBE & 78 & 2.12 & -40 & 20 & 20 & 85 & 2.27 \\
PLD & 97 & 2.12 & -80 & 25 & 16 & 26 & 2.18 \\
RF Sputter & 96 & 2.12 & -40 & 20 & 5.1 & 27 & 2.21 \\
CVD & 70 & 7.06 & -80 & 24 & 6.2 & 4.5 & 2.13 \\
RF Sputter (no buffer) & 102 & 2.12 & -40 & 40 & -- & -- & 2.12 \\
\hline \hline
\end{tabular}

Table B.2: Parameters used for simulating $\delta^{\prime}$ as a function of $\theta_{\mathrm{E}}$ (Figure B.4). *fit parameter

\begin{tabular}{|c|c|c|c|c|c|c|c|}
\hline Parameter & $d$ & $w$ & $\varphi$ & $\varepsilon$ BTO & $\underset{\text { (circle) }}{p}$ & $\begin{array}{c}p \\
\text { (square) }\end{array}$ & $\underset{\text { (slab) }}{p}$ \\
\hline & & & & & {$[\%]$} & {$[\%]$} & {$[\%]$} \\
\hline Value & 1 & 1 & 5 & -- & -- & -- & -- \\
\hline Min Value & -- & -- & -- & 50 & 0 & 0 & 0 \\
\hline Max Value & -- & -- & -- & 660 & 75 & 98 & 99 \\
\hline
\end{tabular}

Table B.3: Parameters used for COMSOL simulations of correction factor $c_{\mathrm{p}}$ for porous structures shown in Figure B.6. Variables included distance between electrodes $d$, lateral periodicity $w$, electric potential drop $\varphi$, porosity $p$, dielectric constant $\varepsilon_{\mathrm{BTO}}$, and pore cross-section shape (circle, square, or slab). 


\section{Note: Simulation Details}

The simulation process modified only slightly the procedure detailed in the Supplementary Text of Ref. [67] and Ref. [85]. Rather than interpolate the measured data, we approximate the two branches of data (Figure B.5) with sigmoidal curves of the form,

$\delta^{\prime}\left(E_{o f f}\right)=2 \delta^{\prime} /\left(1+e^{\left(E_{o f f} \pm E_{c}\right) / d x}\right)-\delta^{\prime}{ }_{s}$,

where $\delta_{\mathrm{s}}$ ' is the maximum rotation, $E_{\mathrm{c}}$ is the coercive field for each sample, and $\mathrm{dx}$ is a slope scaling factor (Table B.2). The domain population for a given electric field can then be estimated by shifting the curve to $E_{\mathrm{c}}=0$. Based on this field-dependent domain population, a weighting factor is used when summing Jones vectors from each of the four distinct domain orientations.

Next, the induced rotation $\delta$ was simulated as a function of electric field orientation (Figure B.4(a)). The orientation of the incident polarization of the linearly polarized light where the maximum in $\delta^{\prime}$ is observed, $\theta_{\mathrm{p}, \max }$, was also measured and simulated (Figure B.4(b)) and is in good agreement with best-fit values. The simulation procedure follows Ref. [85] using the parameters in Table B.2. To choose the best-fit values of r33 and r42 the simulated values were compared with measured values to

minimize the parameter $\chi_{\delta}^{2}=\sum_{\theta_{E}=0^{\circ}}^{90^{\circ}}\left(\left|\delta_{\text {meas }}\right|-\left|\delta_{\text {calc }}\right|\right)$ . Best-fit values of r33 and r42 were fitted as in Figure B.4(c) for the bulk value birefringence $\mathrm{nb}=0.03$, and bulk ratio $\mathrm{r} 13=$ r33/10. Error bars were estimated by repeating the fitting procedure for $n b=0.02$ and 0.04. [67] 


\section{References}

1. G. E. Moore, "Cramming more components onto integrated circuits," 38, (1965).

2. "ITRS Reports - International Technology Roadmap for Semiconductors," http://www.itrs2.net/itrs-reports.html.

3. H. Y. Hwang, "Tuning Interface States," Science 313, 1895-1896 (2006).

4. K. Ueda, H. Tabata, and T. Kawai, "Ferromagnetism in $\mathrm{LaFeO} 3-\mathrm{LaCrO} 3$ Superlattices," Science 280, 1064-1066 (1998).

5. H. Yamada, Y. Ogawa, Y. Ishii, H. Sato, M. Kawasaki, H. Akoh, and Y. Tokura, "Engineered interface of magnetic oxides.," Science 305, 646-8 (2004).

6. J. Chakhalian, J. W. Freeland, H.-U. Habermeier, G. Cristiani, G. Khaliullin, M. van Veenendaal, and B. Keimer, "Orbital reconstruction and covalent bonding at an oxide interface.," Science 318, 1114-7 (2007).

7. J. Hoffman, X. Pan, J. W. Reiner, F. J. Walker, J. P. Han, C. H. Ahn, and T. P. Ma, "Ferroelectric field effect transistors for memory applications.," Adv. Mater. 22, 2957-61 (2010).

8. H. W. Jang, S. H. Baek, D. Ortiz, C. M. Folkman, C. B. Eom, Y. H. Chu, P. Shafer, R. Ramesh, V. Vaithyanathan, and D. G. Schlom, "Epitaxial (001) $\mathrm{BiFeO}_{3}$ membranes with substantially reduced fatigue and leakage," Appl. Phys. Lett. 92, 62910 (2008).

9. S. Maekawa, T. Tohyama, S. E. Barnes, S. Ishihara, W. Koshibae, and G. Khaliullin, Physics of Transition Metal Oxides, Springer Series in Solid-State Sciences (Springer Berlin Heidelberg, 2004), Vol. 144.

10. A. A. Demkov, K. J. Kormondy, and K. D. Fredrickson, "Two-Dimensional Electron Gas at Oxide Interfaces," in Oxide Materials at the Two-Dimensional Limit, F. P. Netzer and A. Fortunelli, eds., Springer Series in Materials Science (Springer International Publishing, 2016), Vol. 234, pp. 335-359.

11. A. Ohtomo and H. Y. Hwang, "A high-mobility electron gas at the $\mathrm{LaAlO}_{3} / \mathrm{SrTiO}_{3}$ heterointerface.," Nature 427, 423-6 (2004).

12. W. Siemons, G. Koster, H. Yamamoto, W. Harrison, G. Lucovsky, T. Geballe, D. Blank, and M. Beasley, "Origin of Charge Density at $\mathrm{LaAlO}_{3}$ on $\mathrm{SrTiO}_{3}$ Heterointerfaces: Possibility of Intrinsic Doping," Phys. Rev. Lett. 98, 196802 (2007). 
13. A. Kalabukhov, R. Gunnarsson, J. Börjesson, E. Olsson, T. Claeson, and D. Winkler, "Effect of oxygen vacancies in the $\mathrm{SrTiO}_{3}$ substrate on the electrical properties of the $\mathrm{LaAlO}_{3} \mathrm{SrTiO}_{3}$ interface," Phys. Rev. B 75, 121404 (2007).

14. L. Qiao, T. Droubay, T. Varga, M. Bowden, V. Shutthanandan, Z. Zhu, T. Kaspar, and S. Chambers, "Epitaxial growth, structure, and intermixing at the $\mathrm{LaAlO}_{3} / \mathrm{SrTiO}_{3}$ interface as the film stoichiometry is varied," Phys. Rev. B 83, 85408 (2011).

15. J. Mundy, L. Fitting Kourkoutis, M. Warusawithana, D. Schlom, and D. Muller, "Probing Stoichiometry in $\mathrm{LaAlO}_{3} / \mathrm{SrTiO}_{3}$ Interfaces by Aberration-Corrected STEM," Microsc. Microanal. 17, 1450-1451 (2011).

16. M. Basletic, J.-L. Maurice, C. Carrétéro, G. Herranz, O. Copie, M. Bibes, E. Jacquet, K. Bouzehouane, S. Fusil, and A. Barthélémy, "Mapping the spatial distribution of charge carriers in $\mathrm{LaAlO}_{3} / \mathrm{SrTiO}_{3}$ heterostructures.," Nat. Mater. 7, 621-5 (2008).

17. G. Khalsa and A. H. MacDonald, "Theory of the $\mathrm{SrTiO}_{3}$ surface state twodimensional electron gas," Phys. Rev. B 86, 125121 (2012).

18. M. Takizawa, H. Wadati, K. Tanaka, M. Hashimoto, T. Yoshida, A. Fujimori, A. Chikamatsu, H. Kumigashira, M. Oshima, K. Shibuya, T. Mihara, T. Ohnishi, M. Lippmaa, M. Kawasaki, H. Koinuma, S. Okamoto, and A. Millis, "Photoemission from Buried Interfaces in $\mathrm{SrTiO}_{3} / \mathrm{LaTiO}_{3}$ Superlattices," Phys. Rev. Lett. 97, 57601 (2006).

19. S. Okamoto, A. Millis, and N. Spaldin, "Lattice Relaxation in Oxide Heterostructures: $\mathrm{LaTiO}_{3} / \mathrm{SrTiO}_{3}$ Superlattices," Phys. Rev. Lett. 97, 56802 (2006).

20. A. Ohtomo, D. A. Muller, J. L. Grazul, and H. Y. Hwang, "Artificial chargemodulationin atomic-scale perovskite titanate superlattices.," Nature 419, 378-80 (2002).

21. R. Pentcheva and W. Pickett, "Avoiding the Polarization Catastrophe in $\mathrm{LaAlO}_{3}$ Overlayers on $\mathrm{SrTiO}_{3}(001)$ through Polar Distortion," Phys. Rev. Lett. 102, 107602 (2009).

22. R. Pentcheva and W. E. Pickett, "Charge localization or itineracy at $\mathrm{LaAlO}_{3} \mathrm{SrTiO}_{3}$ interfaces: Hole polarons, oxygen vacancies, and mobile electrons," Phys. Rev. B 74, 35112 (2006).

23. J. Lee and A. A. Demkov, "Charge origin and localization at the n-type 
$\mathrm{SrTiO}_{3} / \mathrm{LaAlO}_{3}$ interface," Phys. Rev. B 78, 193104 (2008).

24. N. Reyren, S. Thiel, A. D. Caviglia, L. F. Kourkoutis, G. Hammerl, C. Richter, C. W. Schneider, T. Kopp, A.-S. Rüetschi, D. Jaccard, M. Gabay, D. A. Muller, J.-M. Triscone, and J. Mannhart, "Superconducting interfaces between insulating oxides.," Science 317, 1196-9 (2007).

25. A. D. Caviglia, S. Gariglio, N. Reyren, D. Jaccard, T. Schneider, M. Gabay, S. Thiel, G. Hammerl, J. Mannhart, and J.-M. Triscone, "Electric field control of the $\mathrm{LaAlO}_{3} / \mathrm{SrTiO}_{3}$ interface ground state.," Nature 456, 624-7 (2008).

26. A. Manchon and S. Zhang, "Theory of nonequilibrium intrinsic spin torque in a single nanomagnet," Phys. Rev. B 78, 212405 (2008).

27. G. Khalsa, B. Lee, and A. H. MacDonald, "Theory of $t_{2 \mathrm{~g}}$ electron-gas Rashba interactions," Phys. Rev. B 88, 41302 (2013).

28. A. D. Caviglia, M. Gabay, S. Gariglio, N. Reyren, C. Cancellieri, and J.-M. Triscone, "Tunable Rashba Spin-Orbit Interaction at Oxide Interfaces," Phys. Rev. Lett. 104, 126803 (2010).

29. R. Arras, V. G. Ruiz, W. E. Pickett, and R. Pentcheva, "Tuning the twodimensional electron gas at the $\mathrm{LaAlO}_{3} / \mathrm{SrTiO}_{3}(001)$ interface by metallic contacts," Phys. Rev. B 85, 125404 (2012).

30. J. W. Park, D. F. Bogorin, C. Cen, D. a Felker, Y. Zhang, C. T. Nelson, C. W. Bark, C. M. Folkman, X. Q. Pan, M. S. Rzchowski, J. Levy, and C. B. Eom, "Creation of a two-dimensional electron gas at an oxide interface on silicon.," Nat. Commun. 1, 94 (2010).

31. C. Cen, S. Thiel, J. Mannhart, and J. Levy, "Oxide nanoelectronics on demand.," Science 323, 1026-30 (2009).

32. C. Cen, S. Thiel, G. Hammerl, C. W. Schneider, K. E. Andersen, C. S. Hellberg, J. Mannhart, and J. Levy, "Nanoscale control of an interfacial metal-insulator transition at room temperature.," Nat. Mater. 7, 298-302 (2008).

33. J. Goniakowski, F. Finocchi, and C. Noguera, "Polarity of oxide surfaces and nanostructures," Reports Prog. Phys. 71, 16501 (2008).

34. W. Harrison, E. Kraut, J. Waldrop, and R. Grant, "Polar heterojunction interfaces," Phys. Rev. B 18, 4402-4410 (1978).

35. H. Y. Hwang, Y. Iwasa, M. Kawasaki, B. Keimer, N. Nagaosa, and Y. Tokura, "Emergent phenomena at oxide interfaces.," Nat. Mater. 11, 103-13 (2012). 
36. J. N. Eckstein, "Oxide interfaces: watch out for the lack of oxygen.," Nat. Mater. 6, 473-4 (2007).

37. G. Herranz, F. Sánchez, N. Dix, M. Scigaj, and J. Fontcuberta, "High mobility conduction at (110) and (111) $\mathrm{LaAlO}_{3} / \mathrm{SrTiO}_{3}$ interfaces.," Sci. Rep. 2, 758 (2012).

38. Z. Q. Liu, C. J. Li, W. M. Lü, X. H. Huang, Z. Huang, S. W. Zeng, X. P. Qiu, L. S. Huang, A. Annadi, J. S. Chen, J. M. D. Coey, and T. Venkatesan, "Origin of the Two-Dimensional Electron Gas at $\mathrm{LaAlO}_{3} / \mathrm{SrTiO}_{3}$ Interfaces: The Role of Oxygen Vacancies and Electronic Reconstruction," Phys. Rev. X 3, 21010 (2013).

39. L. Yu and A. Zunger, "A unified mechanism for conductivity and magnetism at interfaces of insulating nonmagnetic oxides," Nat. Commun. 5, 5118 (2014).

40. H. Seo and A. A. Demkov, "First-principles study of polar $\mathrm{LaAlO}_{3}(001)$ surface stabilization by point defects," Phys. Rev. B 84, 45440 (2011).

41. M. Weissmann and V. Ferrari, "Ab initio study of a $\mathrm{TiO}_{2} / \mathrm{LaAlO}_{3}$ heterostructure," J. Phys. Conf. Ser. 167, 12060 (2009).

42. V. Ferrari and M. Weissmann, "Tuning the insulator-metal transition in oxide interfaces: An ab initio study exploring the role of oxygen vacancies and cation interdiffusion," Phys. status solidi 251, 1601-1607 (2014).

43. S. Amoruso, C. Aruta, P. Aurino, R. Bruzzese, X. Wang, F. M. Granozio, and U. Scotti di Uccio, "Oxygen background gas influence on pulsed laser deposition process of $\mathrm{LaAlO}_{3}$ and $\mathrm{LaGaO}_{3}$," Appl. Surf. Sci. 258, 9116-9122 (2012).

44. Y. Chen, N. Pryds, J. E. Kleibeuker, G. Koster, J. Sun, E. Stamate, B. Shen, G. Rijnders, and S. Linderoth, "Metallic and insulating interfaces of amorphous $\mathrm{SrTiO}_{3}$-based oxide heterostructures.," Nano Lett. 11, 3774-8 (2011).

45. S. W. Lee, Y. Liu, J. Heo, and R. G. Gordon, "Creation and control of twodimensional electron gas using Al-based amorphous oxides $/ \mathrm{SrTiO}_{3}$ heterostructures grown by atomic layer deposition.," Nano Lett. 12, 4775-83 (2012).

46. B. Wul and I. M. Goldmand, "No Title," C. R. Acad. Sci. URSS 51, (1946).

47. J. Valasek, "Piezo-Electric and Allied Phenomena in Rochelle Salt," Phys. Rev. 17, 475-481 (1921).

48. J. Thurnauer, H., Deaderick, "Insulating Material," (1947).

49. F. Pockels, Lehrbuch Der Kristalloptik (Leipzig und Berlin, 1906). 
50. E. L. Wooten, K. M. Kissa, A. Yi-Yan, E. J. Murphy, D. A. Lafaw, P. F. Hallemeier, D. Maack, D. V. Attanasio, D. J. Fritz, G. J. McBrien, and D. E. Bossi, "A review of lithium niobate modulators for fiber-optic communications systems," IEEE J. Sel. Top. Quantum Electron. 6, 69-82 (2000).

51. A. Chen and E. J. Murphy, Broadband Optical Modulators : Science, Technology, and Applications (CRC Press/Taylor \& Francis Group, 2012).

52. J. W. Goodman, F. J. Leonberger, Sun-Yuan Kung, and R. A. Athale, "Optical interconnections for VLSI systems," Proc. IEEE 72, 850-866 (1984).

53. K. Yamada, Y. Urino, T. Nakamura, and Y. Arakawa, "Integrated silicon-based optical interconnect for fast, compact, energy-efficient electronic circuit systems," NTT Tech. Rev. 11, (2013).

54. D. A. B. Miller, "Optical interconnects to electronic chips," Appl. Opt. 49, F59 (2010).

55. J. Gourlay, M. Forbes, and M. Desmulliez, "Optically interconnected electronic chips: a tutorial and review of the technology," Electron. Commun. Eng. J. 13, 221-232 (2001).

56. A. F. Benner, M. Ignatowski, J. A. Kash, D. M. Kuchta, and M. B. Ritter, "Exploitation of optical interconnects in future server architectures," IBM J. Res. Dev. 49, 755-775 (2005).

57. M. R. Feldman, S. C. Esener, C. C. Guest, and S. H. Lee, "Comparison between optical and electrical interconnects based on power and speed considerations," Appl. Opt. 27, 1742 (1988).

58. P. Yeh, Introduction to Photorefractive Nonlinear Optics (Wiley, 1993).

59. S. I. Association, "2015 ITRS Section 5: More Moore," 1-52 (2015).

60. G. T. Reed, G. Mashanovich, F. Y. Gardes, and D. J. Thomson, "Silicon optical modulators," Nat. Photonics 4, 518-526 (2010).

61. C. Schriever, C. Bohley, J. Schilling, and R. B. Wehrspohn, "Strained Silicon Photonics," Materials (Basel). 5, 889-908 (2012).

62. M. J. R. Heck, H.-W. Chen, A. W. Fang, B. R. Koch, D. Liang, H. Park, M. N. Sysak, and J. E. Bowers, "Hybrid Silicon Photonics for Optical Interconnects," IEEE J. Sel. Top. Quantum Electron. 17, 333-346 (2011).

63. S. Abel, T. Stoferle, C. Marchiori, D. Caimi, L. Czornomaz, M. Stuckelberger, M. 
Sousa, B. J. Offrein, and J. Fompeyrine, "A Hybrid Barium Titanate-Silicon Photonics Platform for Ultraefficient Electro-Optic Tuning," J. Light. Technol. 34, 1688-1693 (2016).

64. K. Vandoorne, P. Mechet, T. Van Vaerenbergh, M. Fiers, G. Morthier, D. Verstraeten, B. Schrauwen, J. Dambre, and P. Bienstman, "Experimental demonstration of reservoir computing on a silicon photonics chip.," Nat. Commun. 5, 3541 (2014)

65. P. Rabiei, J. Ma, S. Khan, J. Chiles, and S. Fathpour, "Heterogeneous lithium niobate photonics on silicon substrates," Opt. Express 21, 114-115 (2013).

66. R. S. Jacobsen, K. N. Andersen, P. I. Borel, J. Fage-Pedersen, L. H. Frandsen, O. Hansen, M. Kristensen, A. V Lavrinenko, G. Moulin, H. Ou, C. Peucheret, B. Zsigri, and A. Bjarklev, "Strained silicon as a new electro-optic material.," Nature 441, 199-202 (2006).

67. S. Abel, T. Stöferle, C. Marchiori, C. Rossel, M. D. Rossell, R. Erni, D. Caimi, M. Sousa, A. Chelnokov, B. J. Offrein, and J. Fompeyrine, "A strong electro-optically active lead-free ferroelectric integrated on silicon," Nat. Commun. 4, 1671 (2013).

68. M. Lorenz, M. S. Ramachandra Rao, T. Venkatesan, E. Fortunato, P. Barquinha, R. Branquinho, D. Salgueiro, R. Martins, E. Carlos, A. Liu, F. K. Shan, M. Grundmann, H. Boschker, J. Mukherjee, M. Priyadarshini, N. DasGupta, D. J. Rogers, F. H. Teherani, E. V Sandana, P. Bove, K. Rietwyk, A. Zaban, A. Veziridis, A. Weidenkaff, M. Muralidhar, M. Murakami, S. Abel, J. Fompeyrine, J. Zuniga-Perez, R. Ramesh, N. A. Spaldin, S. Ostanin, V. Borisov, I. Mertig, V. Lazenka, G. Srinivasan, W. Prellier, M. Uchida, M. Kawasaki, R. Pentcheva, P. Gegenwart, F. Miletto Granozio, J. Fontcuberta, and N. Pryds, "The 2016 oxide electronic materials and oxide interfaces roadmap," J. Phys. D. Appl. Phys. 49, 433001 (2016).

69. C. Merckling, Z. Liu, M. Hsu, S. Hasan, S. Jiang, S. El Kazzi, G. Boccardi, N. Waldron, Z. Wang, B. Tian, M. Pantouvaki, J. Van Campenhout, N. Collaert, M. Heyns, D. Van Thourhout, W. Vandervorst, and A. Thean, "(Invited) III-V Selective Area Growth and Epitaxial Functional Oxides on Si: From Electronic to Photonic Devices," ECS Trans. 72, 59-69 (2016).

70. H. Ma, A. K.-Y. Jen, and L. R. Dalton, "Polymer-Based Optical Waveguides: Materials, Processing, and Devices," Adv. Mater. 14, 1339-1365 (2002).

71. A. Melikyan, L. Alloatti, A. Muslija, D. Hillerkuss, P. C. Schindler, J. Li, R. Palmer, D. Korn, S. Muehlbrandt, D. Van Thourhout, B. Chen, R. Dinu, M. Sommer, C. Koos, M. Kohl, W. Freude, and J. Leuthold, "High-speed plasmonic 
phase modulators," Nat. Photonics 8, 229-233 (2014).

72. C. Xiong, W. H. P. Pernice, J. H. Ngai, J. W. Reiner, D. Kumah, F. J. Walker, C. H. Ahn, and H. X. Tang, "Active Silicon Integrated Nanophotonics: Ferroelectric $\mathrm{BaTiO}_{3}$ Devices," Nano Lett. 14, 1419-1425 (2014).

73. B. Chmielak, M. Waldow, C. Matheisen, C. Ripperda, J. Bolten, T. Wahlbrink, M. Nagel, F. Merget, and H. Kurz, "Pockels effect based fully integrated, strained silicon electro-optic modulator.," Opt. Express 19, 17212-9 (2011).

74. B. W. Wessels, "Ferroelectric Epitaxial Thin Films for Integrated Optics," Annu. Rev. Mater. Res. 37, 659-679 (2007).

75. P. Bernasconi, M. Zgonik, and P. Günter, "Temperature dependence and dispersion of electro-optic and elasto-optic effect in perovskite crystals," J. Appl. Phys. 78, 2651 (1995).

76. F. J. Walker, R. A. McKee, H. Yen, and D. E. Zelmon, "Optical clarity and waveguide performance of thin film perovskites on $\mathrm{MgO}$," Appl. Phys. Lett. 65, 1495-1497 (1994).

77. D. M. Gill, C. W. Conrad, G. Ford, B. W. Wessels, and S. T. Ho, "Thin-film channel waveguide electro-optic modulator in epitaxial $\mathrm{BaTiO}_{3}$," Appl. Phys. Lett. 71, 1783-1785 (1997).

78. P. Tang, A. L. Meier, D. J. Towner, and B. W. Wessels, "BaTiO 3 thin-film waveguide modulator with a low voltage-length product at near-infrared wavelengths of 098 and $155 \mu \mathrm{m}$," Opt. Lett. 30, 254 (2005).

79. R. A. McKee, F. J. Walker, and M. F. Chisholm, "Crystalline Oxides on Silicon: The First Five Monolayers," Phys. Rev. Lett. 81, 3014-3017 (1998).

80. C. Dubourdieu, J. Bruley, T. M. Arruda, A. Posadas, J. Jordan-Sweet, M. M. Frank, E. Cartier, D. J. Frank, S. V Kalinin, A. A. Demkov, and V. Narayanan, "Switching of ferroelectric polarization in epitaxial $\mathrm{BaTiO}_{3}$ films on silicon without a conducting bottom electrode," Nat. Nanotechnol. 8, 748-754 (2013).

81. F. Eltes, D. Caimi, F. Fallegger, M. Sousa, E. O’Connor, M. D. Rossell, B. Offrein, J. Fompeyrine, and S. Abel, "Low-Loss $\mathrm{BaTiO}_{3}-\mathrm{Si}$ Waveguides for Nonlinear Integrated Photonics," ACS Photonics 3, 1698-1703 (2016).

82. A. Y. Cho and J. R. Arthur, "Molecular beam epitaxy," Prog. Solid State Chem. 10, 157-191 (1975).

83. A. Ichimiya and P. I. Cohen, Reflection High-Energy Electron Diffraction 
(Cambridge University Press, 2004).

84. R. C. Hatch, M. Choi, A. B. Posadas, and A. A. Demkov, "Comparison of acidand non-acid-based surface preparations of Nb-doped $\mathrm{SrTiO}_{3}(001)$," J. Vac. Sci. Technol. B, Nanotechnol. Microelectron. Mater. Process. Meas. Phenom. 33, 61204 (2015).

85. S. Abel, D. Caimi, M. Sousa, T. Stöferle, C. Rossel, C. Marchiori, A. Chelnokov, and J. Fompeyrine, "Electro-optical properties of barium titanate films epitaxially grown on silicon," SPIE OPTO 8263, 82630Y (2012).

86. D. H. Reitze, E. Haton, R. Ramesh, S. Etemad, D. E. Leaird, T. Sands, Z. Karim, and A. R. Tanguay, "Electro-optic properties of single crystalline ferroelectric thin films," Appl. Phys. Lett. 63, 596-598 (1993).

87. K. J. Kormondy, A. B. Posadas, A. Slepko, A. Dhamdhere, D. J. Smith, K. N. Mitchell, T. I. Willett-gies, S. Zollner, L. G. Marshall, J. Zhou, and A. A. Demkov, "Epitaxy of polar semiconductor $\mathrm{Co}_{3} \mathrm{O}_{4}$ (110): Growth, structure, and characterization," J. Appl. Phys. 115, 243708 (2014).

88. G. Eranna, B. C. Joshi, D. P. Runthala, and R. P. Gupta, "Oxide Materials for Development of Integrated Gas Sensors-A Comprehensive Review," Crit. Rev. Solid State Mater. Sci. 29, 111-188 (2004).

89. W. Y. Li, L. N. Xu, and J. Chen, " $\mathrm{Co}_{3} \mathrm{O}_{4}$ Nanomaterials in Lithium-Ion Batteries and Gas Sensors," Adv. Funct. Mater. 15, 851-857 (2005).

90. J. A. Moyer, C. A. F. Vaz, D. A. Arena, D. Kumah, E. Negusse, and V. E. Henrich, "Magnetic structure of Fe-doped $\mathrm{CoFe}_{2} \mathrm{O}_{4}$ probed by x-ray magnetic spectroscopies," Phys. Rev. B 84, 54447 (2011).

91. M. S. Martín-González, J. F. Fernández, F. Rubio-Marcos, I. Lorite, J. L. CostaKrämer, A. Quesada, M. A. Bañares, and J. L. G. Fierro, "Insights into the room temperature magnetism of $\mathrm{ZnO} / \mathrm{Co}_{3} \mathrm{O}_{4}$ mixtures," J. Appl. Phys. 103, 83905 (2008).

92. A. Posadas, M. Berg, H. Seo, D. J. Smith, A. P. Kirk, D. Zhernokletov, R. M. Wallace, A. de Lozanne, and A. A. Demkov, "Strain-induced ferromagnetism in $\mathrm{LaCoO}_{3}$ : Theory and growth on $\mathrm{Si}(100), "$ Microelectron. Eng. 88, 1444-1447 (2011).

93. A. Posadas, M. Berg, H. Seo, A. de Lozanne, A. A. Demkov, D. J. Smith, A. P. Kirk, D. Zhernokletov, and R. M. Wallace, "Epitaxial integration of ferromagnetic correlated oxide $\mathrm{LaCoO}_{3}$ with Si (100)," Appl. Phys. Lett. 98, 53104 (2011). 
94. J. Wöllenstein, M. Burgmair, G. Plescher, T. Sulima, J. Hildenbrand, H. Böttner, and I. Eisele, "Cobalt oxide based gas sensors on silicon substrate for operation at low temperatures," Sensors Actuators B Chem. 93, 442-448 (2003).

95. M. M. Natile and A. Glisenti, "Study of surface reactivity of cobalt oxides: Interaction with methanol," Chem. Mater. 14, 3090-3099 (2002).

96. H. Sun, H. M. Ang, M. O. Tadé, and S. Wang, " $\mathrm{Co}_{3} \mathrm{O}_{4}$ nanocrystals with predominantly exposed facets: synthesis, environmental and energy applications," J. Mater. Chem. A 1, 14427 (2013).

97. X. Xie, Y. Li, Z.-Q. Liu, M. Haruta, and W. Shen, "Low-temperature oxidation of $\mathrm{CO}$ catalysed by $\mathrm{Co}_{3} \mathrm{O}_{4}$ nanorods.," Nature 458, 746-9 (2009).

98. F. Jiao and H. Frei, "Nanostructured cobalt oxide clusters in mesoporous silica as efficient oxygen-evolving catalysts.," Angew. Chemie 48, 1841-1844 (2009).

99. A. Agiral, H. S. Soo, and H. Frei, "Visible Light Induced Hole Transport from Sensitizer to $\mathrm{Co}_{3} \mathrm{O}_{4}$ Water Oxidation Catalyst across Nanoscale Silica Barrier with Embedded Molecular Wires," Chem. Mater. 25, 2264-2273 (2013).

100. Y. Liang, Y. Li, H. Wang, J. Zhou, J. Wang, T. Regier, and H. Dai, " $\mathrm{Co}_{3} \mathrm{O}_{4}$ nanocrystals on graphene as a synergistic catalyst for oxygen reduction reaction.," Nat. Mater. 10, 780-6 (2011).

101. T. Q. Ngo, A. Posadas, H. Seo, S. Hoang, M. D. McDaniel, D. Utess, D. H. Triyoso, C. Buddie Mullins, A. A. Demkov, and J. G. Ekerdt, "Atomic layer deposition of photoactive $\mathrm{CoO} / \mathrm{SrTiO}_{3}$ and $\mathrm{CoO} / \mathrm{TiO}_{2}$ on $\mathrm{Si}(001)$ for visible light driven photoelectrochemical water oxidation," J. Appl. Phys. 114, 84901 (2013).

102. S. C. Petitto and M. A. Langell, "Surface composition and structure of $\mathrm{Co}_{3} \mathrm{O}_{4}$ (110) and the effect of impurity segregation," J. Vac. Sci. Technol. A 22, 1690 (2004).

103. Z.-X. Shen, J. W. Allen, P. A. P. Lindberg, D. S. Dessau, B. O. Wells, A. Borg, W. Ellis, J. S. Kang, S.-J. Oh, I. Lindau, and W. E. Spicer, "Photoemission study of CoO," Phys. Rev. B 42, 1817-1828 (1990).

104. M. A. Langell, M. D. Anderson, G. A. Carson, L. Peng, and S. Smith, "Valenceband electronic structure of $\mathrm{Co}_{3} \mathrm{O}_{4}$ epitaxy on $\mathrm{CoO}(100)$," Phys. Rev. B 59, 47914798 (1999).

105. L. Qiao, H. Y. Xiao, H. M. Meyer, J. N. Sun, C. M. Rouleau, A. A. Puretzky, D. B. Geohegan, I. N. Ivanov, M. Yoon, W. J. Weber, and M. D. Biegalski, "Nature of the band gap and origin of the electro-/photo-activity of $\mathrm{Co}_{3} \mathrm{O}_{4}$," J. Mater. 
Chem. C 1, 4628 (2013).

106. K. J. Kim and Y. R. Park, "Optical investigation of charge-transfer transitions in spinel $\mathrm{Co}_{3} \mathrm{O}_{4}$," Solid State Commun. 127, 25-28 (2003).

107. H. Shirai, Y. Morioka, and I. Nakagawa, "Infrared and Raman spectra and lattice vibrations of some oxide spinels," J. Phys. Soc. Jpn 51, 592-597 (1982).

108. G. N. Maslennikova, "Pigments of the spinel type," Glas. Ceram. 58, 216-220 (2001).

109. M. Bouchard and A. Gambardella, "Raman microscopy study of synthetic cobalt blue spinels used in the field of art," J. Raman Spectrosc. 41, 1477-1485 (2010).

110. W. L. Roth, "The magnetic structure of $\mathrm{Co}_{3} \mathrm{O}_{4}$," J. Phys. Chem. Solids 25, 1-10 (1964).

111. P. Dutta, M. S. Seehra, S. Thota, and J. Kumar, "A comparative study of the magnetic properties of bulk and nanocrystalline $\mathrm{Co}_{3} \mathrm{O}_{4}$," J. Phys. Condens. Matter 20, 15218 (2008).

112. T. Mousavand, T. Naka, K. Sato, S. Ohara, M. Umetsu, S. Takami, T. Nakane, A. Matsushita, and T. Adschiri, "Crystal size and magnetic field effects in $\mathrm{Co}_{3} \mathrm{O}_{4}$ antiferromagnetic nanocrystals," Phys. Rev. B 79, 144411 (2009).

113. H. Yoshikawa, K. Hayashida, Y. Kozuka, A. Horiguchi, K. Awaga, S. Bandow, and S. Iijima, "Preparation and magnetic properties of hollow nano-spheres of cobalt and cobalt oxide: Drastic cooling-field effects on remnant magnetization of antiferromagnet," Appl. Phys. Lett. 85, 5287 (2004).

114. C. Noguera, "Polar oxide surfaces," J. Phys. Condens. Matter 12, R367 (2000).

115. U. Diebold, S.-C. Li, and M. Schmid, "Oxide surface science.," Annu. Rev. Phys. Chem. 61, 129-48 (2010).

116. Y. Z. Chen, N. Bovet, F. Trier, D. V. Christensen, F. M. Qu, N. H. Andersen, T. Kasama, W. Zhang, R. Giraud, J. Dufouleur, T. S. Jespersen, J. R. Sun, A. Smith, J. Nygård, L. Lu, B. Büchner, B. G. Shen, S. Linderoth, and N. Pryds, "A highmobility two-dimensional electron gas at the spinel/perovskite interface of $\gamma$ $\mathrm{Al}_{2} \mathrm{O}_{3} / \mathrm{SrTiO}_{3}$.," Nat. Commun. 4, 1371 (2013).

117. W. Meyer, K. Biedermann, M. Gubo, L. Hammer, and K. Heinz, "Surface structure of polar $\mathrm{Co}_{3} \mathrm{O}_{4}$ (111) films grown epitaxially on $\operatorname{Ir}(100)-(1 \times 1)$.," J. Phys. Condens. Matter 20, 265011 (2008). 
118. K. B. Klepper, O. Nilsen, and H. Fjellvåg, "Growth of thin films of $\mathrm{Co}_{3} \mathrm{O}_{4}$ by atomic layer deposition," Thin Solid Films 515, 7772-7781 (2007).

119. M. E. Donders, H. C. M. Knoops, M. C. M. Van, W. M. M. Kessels, and P. H. L. Notten, "Remote Plasma Atomic Layer Deposition of $\mathrm{Co}_{3} \mathrm{O}_{4}$ Thin Films," J. Electrochem. Soc. 158, G92 (2011).

120. K. B. B. Klepper, O. Nilsen, and H. Fjellvåg, "Epitaxial growth of cobalt oxide by atomic layer deposition," J. Cryst. Growth 307, 457-465 (2007).

121. K. Shalini, A. U. Mane, S. A. Shivashankar, M. Rajeswari, and S. Choopun, "Epitaxial growth of $\mathrm{Co}_{3} \mathrm{O}_{4}$ films by low temperature, low pressure chemical vapour deposition," J. Cryst. Growth 231, 242-247 (2001).

122. D. Barreca, A. Devi, R. A. Fischer, D. Bekermann, A. Gasparotto, M. Gavagnin, C. Maccato, E. Tondello, E. Bontempi, L. E. Depero, and C. Sada, "Strongly oriented $\mathrm{Co}_{3} \mathrm{O}_{4}$ thin films on $\mathrm{MgO}(100)$ and $\mathrm{MgAl}_{2} \mathrm{O}_{4}(100)$ substrates by PECVD," CrystEngComm 13, 3670 (2011).

123. E. Fujii, H. Torii, A. Tomozawa, R. Takayama, and T. Hirao, "Preparation of cobalt oxide films by plasma-enhanced metalorganic chemical vapour deposition," J. Mater. Sci. 30, 6013-6018 (1995).

124. A. U. Mane, K. Shalini, and A. Wohlfart, "Strongly oriented thin films of $\mathrm{Co}_{3} \mathrm{O}_{4}$ deposited on single-crystal $\mathrm{MgO}$ (100) by low-pressure, low-temperature MOCVD," J. Cryst. Growth 240, 157-163 (2002).

125. R. J. Kennedy, "The growth of iron oxide, nickel oxide and cobalt oxide thin films by laser ablation from metal targets," IEEE Trans. Magn. 31, 3829-3831 (1995).

126. C. A. F. Vaz, D. Prabhakaran, E. I. Altman, and V. E. Henrich, "Experimental study of the interfacial cobalt oxide in $\mathrm{Co}_{3} \mathrm{O}_{4} / \alpha-\mathrm{Al}_{2} \mathrm{O}_{3}(0001)$ epitaxial films," Phys. Rev. B 80, 155457 (2009).

127. C. A. F. Vaz, H.-Q. Wang, C. H. Ahn, V. E. Henrich, M. Z. Baykara, T. C. Schwendemann, N. Pilet, B. J. Albers, U. D. Schwarz, L. H. Zhang, Y. Zhu, J. Wang, and E. I. Altman, "Interface and electronic characterization of thin epitaxial $\mathrm{Co}_{3} \mathrm{O}_{4}$ films," Surf. Sci. 603, 291-297 (2009).

128. C. A. F. Vaz, E. I. Altman, and V. E. Henrich, "Exchange bias and interface electronic structure in $\mathrm{Ni} / \mathrm{Co}_{3} \mathrm{O}_{4}$ (011)," Phys. Rev. B 81, 104428 (2010).

129. C. J. Zollner, T. I. Willett-Gies, S. Zollner, and S. Choi, "Infrared to vacuumultraviolet ellipsometry studies of spinel $\left(\mathrm{MgAl}_{2} \mathrm{O}_{4}\right)$," Thin Solid Films 571, 689694 (2014). 
130. H. G. Tompkins and W. A. McGahan, Spectroscopic Ellipsometry and Reflectometry: A User's Guide (Wiley, 1999).

131. H. Fujiwara, Spectroscopic Ellipsometry (Wiley, 2007).

132. H. Zheng, M. Gruyters, E. Pehlke, and R. Berndt, "“Magic" Vicinal Zinc Oxide Surfaces," Phys. Rev. Lett. 111, 86101 (2013).

133. S. A. Chambers, T. Ohsawa, C. M. Wang, I. Lyubinetsky, and J. E. Jaffe, "Band offsets at the epitaxial anatase $\mathrm{TiO}_{2} / \mathrm{n}-\mathrm{SrTiO}_{3}$ (001) interface," Surf. Sci. 603, 771780 (2009).

134. R. C. Hatch and H. Höchst, "Evolution of interface properties of the Pentacene/Bi(0001) system," Surf. Sci. 604, 1684-1687 (2010).

135. S. A. Chambers, Y. Liang, Z. Yu, R. Droopad, J. Ramdani, and K. Eisenbeiser, "Band discontinuities at epitaxial $\mathrm{SrTiO}_{3} / \mathrm{Si}(001)$ heterojunctions," Appl. Phys. Lett. 77, 1662 (2000).

136. X.-L. Xu, Z.-H. Chen, Y. Li, W.-K. Chen, and J.-Q. Li, "Bulk and surface properties of spinel $\mathrm{Co}_{3} \mathrm{O}_{4}$ by density functional calculations," Surf. Sci. 603, 653658 (2009).

137. J. Chen, $\mathrm{X}$. Wu, and A. Selloni, "Electronic structure and bonding properties of cobalt oxide in the spinel structure," Phys. Rev. B 83, 245204 (2011).

138. J. Chen and A. Selloni, "Electronic states and magnetic structure at the $\mathrm{Co}_{3} \mathrm{O}_{4}$ (110) surface: A first-principles study," Phys. Rev. B 85, 85306 (2012).

139. J. C. Green and P. Decleva, "Photoionization cross-sections: a guide to electronic structure," Coord. Chem. Rev. 249, 209-228 (2005).

140. M. Lenglet and C. K. Jørgensen, "Reinvestigation of the optical properties of Co3O4," Chem. Phys. Lett. 229, 616-620 (1994).

141. E. M. Marsh, S. C. Petitto, G. S. Harbison, K. W. Wulser, and M. A. Langell, "Deconvolution of the $\mathrm{Co}_{3} \mathrm{O}_{4}$ (110) Fuchs-Kliewer phonon spectrum," J. Vac. Sci. Technol. A 23, 1061 (2005).

142. M. Khalid, A. Setzer, M. Ziese, P. Esquinazi, D. Spemann, A. Pöppl, and E. Goering, "Ubiquity of ferromagnetic signals in common diamagnetic oxide crystals," Phys. Rev. B 81, 214414 (2010).

143. D. Bloch, "The 103 law for the volume dependence of superexchange," J. Phys. Chem. Solids 27, 881-885 (1966). 
144. Y. Ikedo, J. Sugiyama, H. Nozaki, K. Mukai, H. Itahara, P. L. Russo, D. Andreica, and A. Amato, "High pressure study on cobalt oxide spinel," Phys. B Condens. Matter 404, 652-655 (2009).

145. K. J. Kormondy, A. B. Posadas, T. Q. Ngo, S. Lu, N. Goble, J. Jordan-Sweet, X. P. A. Gao, D. J. Smith, M. R. McCartney, J. G. Ekerdt, and A. A. Demkov, "Quasitwo-dimensional electron gas at the epitaxial alumina/ $/ \mathrm{SrTiO}_{3}$ interface: Control of oxygen vacancies," J. Appl. Phys. 117, 95303 (2015).

146. E. Y. Tsymbal, E. R. A. Dagotto, C.-B. Eom, and R. Ramamoorthy, Multifunctional Oxide Heterostructures (Oxford University Press, 2012).

147. A. A. Demkov and A. B. Posadas, Integration of Functional Oxides with Semiconductors (Springer New York, 2014).

148. C. Hu, K. W. Park, A. Posadas, J. L. Jordan-Sweet, A. A. Demkov, and E. T. Yu, "Voltage-controlled ferromagnetism and magnetoresistance in $\mathrm{LaCoO}_{3} / \mathrm{SrTiO}_{3}$ heterostructures," J. Appl. Phys. 114, 183909 (2013).

149. C. B. Eom, "Epitaxial thin films of isotropic conductive oxides for devices," JOM 49, 47-50 (1997).

150. B. T. Liu, K. Maki, Y. So, V. Nagarajan, R. Ramesh, J. Lettieri, J. H. Haeni, D. G. Schlom, W. Tian, X. Q. Pan, F. J. Walker, and R. A. McKee, "Epitaxial La-doped $\mathrm{SrTiO}_{3}$ on silicon: A conductive template for epitaxial ferroelectrics on silicon," Appl. Phys. Lett. 80, 4801 (2002).

151. K. Ueno, S. Nakamura, H. Shimotani, A. Ohtomo, N. Kimura, T. Nojima, H. Aoki, Y. Iwasa, and M. Kawasaki, "Electric-field-induced superconductivity in an insulator.," Nat. Mater. 7, 855-8 (2008).

152. J. Mannhart and D. G. Schlom, "Oxide interfaces--an opportunity for electronics.," Science 327, 1607-1611 (2010).

153. P. Zubko, S. Gariglio, M. Gabay, P. Ghosez, and J.-M. Triscone, "Interface Physics in Complex Oxide Heterostructures," Annu. Rev. Condens. Matter Phys. 2, 141-165 (2011).

154. N. Pavlenko, T. Kopp, E. Y. Tsymbal, J. Mannhart, and G. A. Sawatzky, "Oxygen vacancies at titanate interfaces: Two-dimensional magnetism and orbital reconstruction," Phys. Rev. B 86, 64431 (2012).

155. Y. Z. Chen, N. Bovet, T. Kasama, W. W. Gao, S. Yazdi, C. Ma, N. Pryds, and S. Linderoth, "Room temperature formation of high-mobility two-dimensional electron gases at crystalline complex oxide interfaces.," Adv. Mater. 26, 1462-7 
(2014).

156. S. W. Lee, J. Heo, and R. G. Gordon, "Origin of the self-limited electron densities at $\mathrm{Al}_{2} \mathrm{O}_{3} / \mathrm{SrTiO}_{3}$ heterostructures grown by atomic layer deposition - oxygen diffusion model.," Nanoscale 5, 8940-4 (2013).

157. C. Lin and A. A. Demkov, "Electron Correlation in Oxygen Vacancy in $\mathrm{SrTiO}_{3}$," Phys. Rev. Lett. 111, 217601 (2013).

158. C. Mitra, C. Lin, J. Robertson, and A. A. Demkov, "Electronic structure of oxygen vacancies in $\mathrm{SrTiO}_{3}$ and $\mathrm{LaAlO}_{3}$," Phys. Rev. B 86, 155105 (2012).

159. T. Nabatame, T. Yasuda, M. Nishizawa, M. Ikeda, T. Horikawa, and A. Toriumi, "Comparative Studies on Oxygen Diffusion Coefficients for Amorphous and $\gamma$ $\mathrm{Al}_{2} \mathrm{O}_{3}$ Films using 18 O Isotope," Jpn. J. Appl. Phys. 42, 7205-7208 (2003).

160. Y. Oishi and W. D. Kingery, "Self-Diffusion of Oxygen in Single Crystal and Polycrystalline Aluminum Oxide," J. Chem. Phys. 33, 480 (1960).

161. M. Choi, A. B. Posadas, C. A. Rodriguez, A. O’Hara, H. Seinige, A. J. Kellock, M. M. Frank, M. Tsoi, S. Zollner, V. Narayanan, and A. A. Demkov, "Structural, optical, and electrical properties of strained La-doped $\mathrm{SrTiO}_{3}$ films," J. Appl. Phys. 116, 43705 (2014).

162. T. Riste, E. J. Samuelsen, K. Otnes, and J. Feder, "Critical behaviour of $\mathrm{SrTiO}_{3}$ near the $105^{\circ} \mathrm{K}$ phase transition," Solid State Commun. 9, 1455-1458 (1971).

163. C. Merckling, M. El-Kazzi, G. Saint-Girons, G. Hollinger, L. Largeau, G. Patriarche, V. Favre-Nicolin, and O. Marty, "Growth of crystalline $\gamma-\mathrm{Al}_{2} \mathrm{O}_{3}$ on $\mathrm{Si}$ by molecular beam epitaxy: Influence of the substrate orientation," J. Appl. Phys. 102, 24101 (2007).

164. C. Merckling, M. El-Kazzi, V. Favre-Nicolin, M. Gendry, Y. Robach, G. Grenet, and G. Hollinger, "Epitaxial growth and relaxation of $\gamma-\mathrm{Al}_{2} \mathrm{O}_{3}$ on silicon," Thin Solid Films 515, 6479-6483 (2007).

165. L. Qiao, T. C. Droubay, T. C. Kaspar, P. V Sushko, and S. A. Chambers, "Cation mixing, band offsets and electric fields at $\mathrm{LaAlO}_{3} / \mathrm{SrTiO}_{3}(001)$ heterojunctions with variable La:Al atom ratio," Surf. Sci. 605, 1381-1387 (2011).

166. S. A. Chambers, L. Qiao, T. C. Droubay, T. C. Kaspar, B. W. Arey, and P. V. Sushko, "Band Alignment, Built-In Potential, and the Absence of Conductivity at the $\mathrm{LaCrO}_{3} / \mathrm{SrTiO}_{3}$ (001) Heterojunction," Phys. Rev. Lett. 107, 206802 (2011).

167. J. A. Rotole, "Gamma-Alumina $\left(\gamma-\mathrm{Al}_{2} \mathrm{O}_{3}\right)$ by XPS," Surf. Sci. Spectra 5, 18 
(1998).

168. J. G. Bednorz and K. A. Müller, "Possible high Tc superconductivity in the Ba-LaCu-O system," Zeitschrift für Phys. B Condens. Matter 64, 189-193 (1986).

169. R. Von Helmolt, J. Wecker, B. Holzapfel, L. Schultz, and K. Samwer, "Giant negative magnetoresistance in perovskitelike $\mathrm{La}_{2 / 3} \mathrm{Ba}_{1 / 3} \mathrm{MnO}_{\mathrm{x}}$ ferromagnetic films," Phys. Rev. Lett. 71, 2331-2333 (1993).

170. J. B. Goodenough, "Theory of the role of covalence in the perovskite-type manganites [La,M(II)] $\mathrm{MnO}_{3}$," Phys. Rev. 100, 564-573 (1955).

171. R. E. Cohen, "Origin of ferroelectricity in perovskite oxides," Nature 358, 136138 (1992).

172. W. Eerenstein, N. D. Mathur, and J. F. Scott, "Multiferroic and magnetoelectric materials," Nature 442759, 759-765 (2006).

173. Y. Saito, H. Takao, T. Tani, T. Nonoyama, K. Takatori, T. Homma, T. Nagaya, and M. Nakamura, "Lead-free piezoceramics," Nature 432, 84-87 (2004).

174. H. Ohta, S. Kim, Y. Mune, T. Mizoguchi, K. Nomura, S. Ohta, T. Nomura, Y. Nakanishi, Y. Ikuhara, M. Hirano, H. Hosono, and K. Koumoto, "Giant thermoelectric Seebeck coefficient of a two-dimensional electron gas in $\mathrm{SrTiO}_{3}$," Nat. Mater. 6, 129-34 (2007).

175. J. Schoenes and P. Wachter, "Exchange optics in Gd-doped EuO," Phys. Rev. B 9, 3097-3105 (1974).

176. P. G. Steeneken, L. H. Tjeng, I. Elfimov, G. A. Sawatzky, G. Ghiringhelli, N. B. Brookes, and D.-J. Huang, "Exchange Splitting and Charge Carrier Spin Polarization in EuO," Phys. Rev. Lett. 88, 47201 (2002).

177. P. M. Tedrow, J. E. Tkaczyk, and A. Kumar, "Spin-Polarized Electron Tunneling Study of an Artificially Layered Superconductor with Internal Magnetic Field: EuO-Al," Phys. Rev. Lett. 56, 1746-1749 (1986).

178. Y. G. Semenov, K. W. Kim, and J. M. Zavada, "Spin field effect transistor with a graphene channel," Appl. Phys. Lett. 91, 153105 (2007).

179. J. Qi, X. Li, Q. Niu, and J. Feng, "Giant and tunable valley degeneracy splitting in $\mathrm{MoTe}_{2}$," Phys. Rev. B - Condens. Matter Mater. Phys. 92, 1-5 (2015).

180. Y. Wang, M. K. Niranjan, J. D. Burton, J. M. An, K. D. Belashchenko, and E. Y. Tsymbal, "Prediction of a spin-polarized two-dimensional electron gas at the 
$\mathrm{LaAlO}_{3} / \mathrm{EuO}$ (001) interface," Phys. Rev. B 79, 212408 (2009).

181. J. Lee, N. Sai, and A. A. Demkov, "Spin-polarized two-dimensional electron gas through electrostatic doping in $\mathrm{LaAlO}_{3} / \mathrm{EuO}$ heterostructures," Phys. Rev. B 82, 235305 (2010).

182. L. B. Pankratz, Thermodynamic Properties of Elements and Oxides / (U.S. Dept. of the Interior, Bureau of Mines :, 1982).

183. M. Choi, C. Lin, M. Butcher, C. Rodriguez, Q. He, A. B. Posadas, A. Y. Borisevich, S. Zollner, and A. A. Demkov, "Quantum confinement in transition metal oxide quantum wells," Appl. Phys. Lett. 106, 192902 (2015).

184. A. B. Posadas, K. J. Kormondy, W. Guo, P. Ponath, J. Geler-Kremer, T. Hadamek, and A. A. Demkov, "Scavenging of oxygen from $\mathrm{SrTiO}_{3}$ during oxide thin film deposition and the formation of interfacial 2DEGs," J. Appl. Phys. 121, 105302 (2017).

185. K. J. Kormondy, S. Abel, F. Fallegger, Y. Popoff, P. Ponath, A. B. Posadas, M. Sousa, D. Caimi, H. Siegwart, E. Uccelli, L. Czornomaz, C. Marchiori, J. Fompeyrine, and A. A. Demkov, "Analysis of the Pockels effect in ferroelectric barium titanate thin films on Si(001)," Microelectron. Eng. 147, 215-218 (2015).

186. J. P. Perdew, K. Burke, and M. Ernzerhof, "Generalized Gradient Approximation Made Simple," Phys. Rev. Lett. 77, 3865-3868 (1996).

187. P. E. Blöchl, "Projector augmented-wave method," Phys. Rev. B 50, 17953 (1994).

188. G. Kresse and J. Furthmüller, "Efficient iterative schemes for ab initio total-energy calculations using a plane-wave basis set," Phys. Rev. B 54, 11169-11186 (1996).

189. S. L. Dudarev, S. Y. Savrasov, C. J. Humphreys, and a. P. Sutton, "Electronenergy-loss spectra and the structural stability of nickel oxide: An LSDA+U study," Phys. Rev. B 57, 1505-1509 (1998).

190. H. J. Monkhorst and J. D. Pack, "Special points for Brillouin-zone integrations," Phys. Rev. B 13, 5188-5192 (1976).

191. V. N. Strocov, X. Wang, M. Shi, M. Kobayashi, J. Krempasky, C. Hess, T. Schmitt, and L. Patthey, "Soft-X-ray ARPES facility at the ADRESS beamline of the SLS: Concepts, technical realisation and scientific applications," J. Synchrotron Radiat. 21, 32-44 (2014).

192. V. N. Strocov, T. Schmitt, U. Flechsig, T. Schmidt, A. Imhof, Q. Chen, J. Raabe, R. Betemps, D. Zimoch, J. Krempasky, X. Wang, M. Grioni, A. Piazzalunga, L. 
Patthey, D. B., and P. L., "High-resolution soft X-ray beamline ADRESS at the Swiss Light Source for resonant inelastic X-ray scattering and angle-resolved photoelectron spectroscopies," J. Synchrotron Radiat. 17, 631-643 (2010).

193. J. Braun, J. Minár, S. Mankovsky, V. N. Strocov, N. B. Brookes, L. Plucinski, C. M. Schneider, C. S. Fadley, and H. Ebert, "Exploring the XPS limit in soft and hard X-ray angle-resolved photoemission using a temperature-dependent one-step theory," Phys. Rev. B 88, 205409 (2013).

194. T. Mairoser, J. A. Mundy, A. Melville, D. Hodash, P. Cueva, R. Held, A. Glavic, J. Schubert, D. A. Muller, D. G. Schlom, and A. Schmehl, "High-quality EuO thin films the easy way via topotactic transformation," Nat. Commun. 6, 7716 (2015).

195. S. Arajs and R. V. Colvin, "Paramagnetic Susceptibility of $\mathrm{Eu}_{2} \mathrm{O}_{3}$ from $300^{\circ}$ to $1300^{\circ}$ K," J. Appl. Phys. 35, 1181-1183 (1964).

196. P. Villars and K. Cenzual, eds., "EuO Crystal Structure: Datasheet from "PAULING FILE Multinaries Edition - 2012" in SpringerMaterials (http://materials.springer.com/isp/crystallographic/docs/sd_0553841)," (n.d.).

197. D. A. Muller, N. Nakagawa, A. Ohtomo, J. L. Grazul, and H. Y. Hwang, "Atomicscale imaging of nanoengineered oxygen vacancy profiles in $\mathrm{SrTiO}_{3}$," Nature 430, 657-61 (2004).

198. Y. Onose, N. Takeshita, C. Terakura, H. Takagi, and Y. Tokura, "Doping dependence of transport properties in $\mathrm{Fe}_{1-\mathrm{x}} \mathrm{Co}_{\mathrm{x}} \mathrm{Si}$," Phys. Rev. B 72, 224431 (2005).

199. H. X. Yang, A. Hallal, D. Terrade, and X. Waintal, "Proximity Effects Induced in Graphene by Magnetic Insulators: First-Principles Calculations on Spin Filtering and Exchange-Splitting Gaps," Phys. Rev. Lett. 46603, 1-5 (2013).

200. D. Ghosh, M. De, and S. De, "Electronic structure and magneto-optical properties of magnetic semiconductors: Europium monochalcogenides," Phys. Rev. B 70, 17 (2004).

201. C. Lin, C. Mitra, and A. A. Demkov, "Orbital ordering under reduced symmetry in transition metal perovskites: Oxygen vacancy in $\mathrm{SrTiO}_{3}$," Phys. Rev. B 86, 161102 (2012).

202. A. A. Demkov, L. R. C. Fonseca, E. Verret, J. Tomfohr, and O. F. Sankey, "Complex band structure and the band alignment problem at the Si high-k dielectric interface," Phys. Rev. B - Condens. Matter Mater. Phys. 71, 1-8 (2005).

203. Z. Hou and K. Terakura, "Defect states induced by oxygen vacancies in cubic 
$\mathrm{SrTiO}_{3}$ : first-principles calculations," J. Phys. Soc. Japan 79, 1-6 (2010).

204. P. A. Lee and T. V. Ramakrishnan, "Disordered electronic systems," Rev. Mod. Phys. 57, 287-337 (1985).

205. A. Gerber, I. Kishon, I. Y. Korenblit, O. Riss, A. Segal, M. Karpovski, and B. Raquet, "Linear Positive Magnetoresistance and Quantum Interference in Ferromagnetic Metals," Phys. Rev. Lett. 99, 27201 (2007).

206. C. Cancellieri, M. L. Reinle-Schmitt, M. Kobayashi, V. N. Strocov, T. Schmitt, P. R. Willmott, S. Gariglio, and J. M. Triscone, "Interface fermi states of $\mathrm{LaAlO}_{3} / \mathrm{SrTiO}_{3}$ and related heterostructures," Phys. Rev. Lett. 110, 137601 (2013).

207. C. Cancellieri, A. S. Mishchenko, U. Aschauer, A. Filippetti, C. Faber, O. S. Barišić, V. A. Rogalev, T. Schmitt, N. Nagaosa, and V. N. Strocov, "Polaronic metal state at the $\mathrm{LaAlO}_{3} / \mathrm{SrTiO}_{3}$ interface," Nat. Commun. 7, 10386 (2016).

208. Z. Wang, S. McKeown Walker, A. Tamai, Y. Wang, Z. Ristic, F. Y. Bruno, A. de la Torre, S. Riccò, N. C. Plumb, M. Shi, P. Hlawenka, J. Sánchez-Barriga, A. Varykhalov, T. K. Kim, M. Hoesch, P. D. C. King, W. Meevasana, U. Diebold, J. Mesot, B. Moritz, T. P. Devereaux, M. Radovic, and F. Baumberger, "Tailoring the nature and strength of electron-phonon interactions in the $\mathrm{SrTiO}_{3}(001) 2 \mathrm{D}$ electron liquid," Nat. Mater. 15, 835-839 (2016).

209. L. Dudy, M. Sing, P. Scheiderer, J. D. Denlinger, P. Schütz, J. Gabel, M. Buchwald, C. Schlueter, T.-L. Lee, and R. Claessen, "In Situ Control of Separate Electronic Phases on $\mathrm{SrTiO}_{3}$ Surfaces by Oxygen Dosing," Adv. Mater. 28, 74437449 (2016).

210. V. N. Strocov, C. Cancellieri, and A. S. Mishchenko, "Electrons and polarons at oxide interfaces explored by soft-X-ray ARPES," (2016).

211. R. A. McKee, F. J. Walker, and M. F. Chisholm, "Physical structure and inversion charge at a semiconductor interface with a crystalline oxide.," Science 293, 468471 (2001).

212. V. Vaithyanathan, J. Lettieri, W. Tian, A. Sharan, A. Vasudevarao, Y. L. Li, A. Kochhar, H. Ma, J. Levy, P. Zschack, J. C. Woicik, L. Q. Chen, V. Gopalan, and D. G. Schlom, "c-axis oriented epitaxial $\mathrm{BaTiO}_{3}$ films on (001) Si," J. Appl. Phys. 100, 24108 (2006).

213. F. Niu and B. W. Wessels, "Epitaxial growth and strain relaxation of $\mathrm{BaTiO}_{3}$ thin films on $\mathrm{SrTiO}_{3}$ buffered (001) $\mathrm{Si}$ by molecular beam epitaxy," J. Vac. Sci. Technol. B Microelectron. Nanom. Struct. 25, 1053 (2007). 
214. R. A. McKee, F. J. Walker, J. R. Conner, E. D. Specht, and D. E. Zelmon, "Molecular beam epitaxy growth of epitaxial barium silicide, barium oxide, and barium titanate on silicon," Appl. Phys. Lett. 59, 782 (1991).

215. R. Pan, Y. He, M. Li, P. Li, P. Liu, and Z. Xia, "Resistive switching in epitaxial $\mathrm{BaTiO}_{3}$ films grown on Nb-doped $\mathrm{SrTiO}_{3}$ by PLD," Mater. Sci. Eng. B 188, 84-88 (2014).

216. A. Barbier, C. Mocuta, D. Stanescu, P. Jegou, N. Jedrecy, and H. Magnan, "Surface composition of $\mathrm{BaTiO}_{3} / \mathrm{SrTiO}_{3}(001)$ films grown by atomic oxygen plasma assisted molecular beam epitaxy," J. Appl. Phys. 112, 114116 (2012).

217. R. C. Hatch, K. D. Fredrickson, M. Choi, C. Lin, H. Seo, A. B. Posadas, and A. A. Demkov, "Surface electronic structure for various surface preparations of $\mathrm{Nb}$ doped $\mathrm{SrTiO}_{3}$ (001)," J. Appl. Phys. 114, 103710 (2013).

218. C. Raisch, T. Chassé, C. Langheinrich, and A. Chassé, "Preparation and investigation of the A-site and B-site terminated $\mathrm{SrTiO}_{3}(001)$ surface: A combined experimental and theoretical x-ray photoelectron diffraction study," J. Appl. Phys. 112, 73505 (2012).

219. G. T. Reed and C. E. Jason Png, "Silicon optical modulators," Mater. Today 8, 4050 (2005).

220. P. Rabiei and P. Gunter, "Optical and electro-optical properties of submicrometer lithium niobate slab waveguides prepared by crystal ion slicing and wafer bonding," Appl. Phys. Lett. 85, 4603 (2004).

221. K. J. Kormondy, Y. Popoff, M. Sousa, F. Eltes, D. Caimi, M. D. Rossell, M. Fiebig, P. Hoffmann, C. Marchiori, M. Reinke, M. Trassin, A. A. Demkov, J. Fompeyrine, and S. Abel, "Microstructure and ferroelectricity of $\mathrm{BaTiO}_{3}$ thin films on Si for integrated photonics," Nanotechnology 28, 75706 (2017).

222. B. E. A. Saleh and M. C. Teich, Fundamentals of Photonics, Wiley Series in Pure and Applied Optics (Wiley, 1991).

223. S. Abel, M. Sousa, C. Rossel, D. Caimi, M. D. Rossell, R. Erni, J. Fompeyrine, and C. Marchiori, "Controlling tetragonality and crystalline orientation in $\mathrm{BaTiO}_{3}$ nano-layers grown on Si," Nanotechnology 24, 285701 (2013).

224. C. Marchiori, M. Sousa, A. Guiller, H. Siegwart, J.-P. Locquet, J. Fompeyrine, G. J. Norga, and J. W. Seo, "Thermal stability of the $\mathrm{SrTiO}_{3}(\mathrm{Ba}, \mathrm{Sr}) \mathrm{O}$ stacks epitaxially grown on Si," Appl. Phys. Lett. 88, 72913 (2006).

225. M. Reinke, Y. Kuzminykh, and P. Hoffmann, "Combinatorial HV-CVD survey of 137 
barium triisopropyl cyclopentadienyl and titanium tetraisopropoxide for the deposition of $\mathrm{BaTiO}_{3}$," Phys. status solidi 212, 1556-1562 (2015).

226. M. Veithen, X. Gonze, and P. Ghosez, "Nonlinear optical susceptibilities, Raman efficiencies, and electro-optic tensors from first-principles density functional perturbation theory," Phys. Rev. B 71, 125107 (2005).

227. K. J. Choi, M. Biegalski, Y. L. Li, A. Sharan, J. Schubert, R. Uecker, P. Reiche, Y. B. Chen, X. Q. Pan, V. Gopalan, L.-Q. Chen, D. G. Schlom, and C. B. Eom, "Enhancement of ferroelectricity in strained $\mathrm{BaTiO}_{3}$ thin films.," Science 306, 1005-1009 (2004).

228. J. Ravez, C. Broustera, and A. Simon, "Lead-free ferroelectric relaxor ceramics in the $\mathrm{BaTiO}_{3}-\mathrm{BaZrO}_{3}-\mathrm{CaTiO}_{3}$ system," J. Mater. Chem. 9, 1609-1613 (1999).

229. E. D. Palik, Handbook of Optical Constants of Solids II (Academic Press, 1991).

230. F. Jona and G. Shirane, Ferroelectric Crystals (Pergamon Press, 1962).

231. D. J. Michalak, J. M. Blackwell, J. M. Torres, A. Sengupta, L. E. Kreno, J. S. Clarke, and D. Pantuso, "Porosity scaling strategies for low-k films," J. Mater. Res. 30, 3363-3385 (2015).

232. F. Ambriz-Vargas, I. Velasco-Davalos, R. Thomas, and A. Ruediger, "Nucleation and growth of ultrathin $\mathrm{BaTiO}_{3}$ films on single terminated $\mathrm{Nb}: \mathrm{SrTiO}_{3}(100)$ substrates for ferroelectric tunnel junctions," J. Vac. Sci. Technol. B 34, 02M101 (2016).

233. L. A. Wills, B. W. Wessels, D. S. Richeson, and T. J. Marks, "Epitaxial growth of $\mathrm{BaTiO}_{3}$ thin films by organometallic chemical vapor deposition," Appl. Phys. Lett. 60, 41 (1992).

234. T. Q. Ngo, A. B. Posadas, M. D. McDaniel, C. Hu, J. Bruley, E. T. Yu, A. A. Demkov, and J. G. Ekerdt, "Epitaxial c-axis oriented $\mathrm{BaTiO}_{3}$ thin films on $\mathrm{SrTiO}_{3}$ buffered Si(001) by atomic layer deposition," Appl. Phys. Lett. 104, 82910 (2014).

235. M. D. McDaniel, A. Posadas, T. Q. Ngo, A. Dhamdhere, D. J. Smith, A. A. Demkov, and J. G. Ekerdt, "Epitaxial strontium titanate films grown by atomic layer deposition on $\mathrm{SrTiO}_{3}$-buffered $\mathrm{Si}(001)$ substrates," J. Vac. Sci. Technol. A 31, 01A136 (2013).

236. J. Y. Li, R. C. Rogan, E. Üstündag, and K. Bhattacharya, "Domain switching in polycrystalline ferroelectric ceramics," Nat. Mater. 4, 776-781 (2005).

237. C. M. Brooks, R. B. Wilson, A. Schäfer, J. A. Mundy, M. E. Holtz, D. A. Muller, 138 
J. Schubert, D. G. Cahill, and D. G. Schlom, "Tuning thermal conductivity in homoepitaxial $\mathrm{SrTiO}_{3}$ films via defects," Appl. Phys. Lett. 107, 51902 (2015).

238. J. W. Park, S. H. Baek, C. W. Bark, M. D. Biegalski, and C. B. Eom, "Quasisingle-crystal (001) $\mathrm{SrTiO}_{3}$ templates on Si," Appl. Phys. Lett. 95, 61902 (2009).

239. L. Zhang, Y. Wang, and R. Engel-Herbert, "Improving the structural quality and electrical resistance of $\mathrm{SrTiO}_{3}$ thin films on $\mathrm{Si}$ (001) via a two-step anneal," J. Appl. Phys. 119, 45301 (2016).

240. M. Zgonik, P. Bernasconi, M. Duelli, R. Schlesser, P. Günter, M. H. Garrett, D. Rytz, Y. Zhu, and X. Wu, "Dielectric, elastic, piezoelectric, electro-optic, and elasto-optic tensors of $\mathrm{BaTiO}_{3}$ crystals," Phys. Rev. B 50, 5941-5949 (1994).

241. N. F. Borrelli and M. M. Layton, "Electrooptic properties of transparent ferroelectric glass-Ceramic systems," IEEE Trans. Electron Devices 16, 511-514 (1969).

242. G. H. Haertling, "Ferroelectric Ceramics: History and Technology," J. Am. Ceram. Soc. 82, 797-818 (1999).

243. D. Damjanovic, "Hysteresis in piezoelectric and ferroelectric materials," Sci. Hysteresis 3, 337-465 (2006).

244. W. J. Merz, "Switching Time in Ferroelectric $\mathrm{BaTiO}_{3}$ and Its Dependence on Crystal Thickness," J. Appl. Phys. 27, 938 (1956).

245. H. A. Lu, L. A. Wills, and B. W. Wessels, "Electrical properties and poling of $\mathrm{BaTiO}_{3}$ thin films," Appl. Phys. Lett. 64, 2973 (1994).

246. K. Okazaki and K. Nagata, "Effects of Grain Size and Porosity on Electrical and Optical Properties of PLZT Ceramics," J. Am. Ceram. Soc. 56, 82-86 (1973).

247. G. Niu, S. Yin, G. Saint-Girons, B. Gautier, P. Lecoeur, V. Pillard, G. Hollinger, and $\mathrm{B}$. Vilquin, "Epitaxy of $\mathrm{BaTiO}_{3}$ thin film on $\mathrm{Si}(001)$ using a $\mathrm{SrTiO}_{3}$ buffer layer for non-volatile memory application," Microelectron. Eng. 88, 1232-1235 (2011).

248. H. A. Lu, L. A. Wills, B. W. Wessels, W. P. Lin, T. G. Zhang, G. K. Wong, D. A. Neumayer, and T. J. Marks, "Second-harmonic generation of poled $\mathrm{BaTiO}_{3}$ thin films," Appl. Phys. Lett. 62, 1314 (1993).

249. N. Ortega, A. Kumar, J. F. Scott, D. B. Chrisey, M. Tomazawa, S. Kumari, D. G. B. Diestra, and R. S. Katiyar, "Relaxor-ferroelectric superlattices: high energy density capacitors," J. Phys. Condens. Matter 24, 445901 (2012). 
250. G. A. Samara, "The relaxational properties of compositionally disordered $\mathrm{ABO}_{3}$ perovskites," J. Phys. Condens. Matter 15, R367-R411 (2003).

251. G. H. Haertling, "PLZT electrooptic materials and applications - a review," Ferroelectrics 75, 25-55 (1987).

252. M. C. Soriano, S. Ortín, D. Brunner, L. Larger, C. R. Mirasso, I. Fischer, and L. Pesquera, "Optoelectronic reservoir computing: tackling noise-induced performance degradation," Opt. Express 21, 12 (2013).

253. L. Appeltant, "Reservoir Computing Based On Delay-Dynamical Systems," (2012).

254. A. Gold, "Scattering time and single-particle relaxation time in a disordered twodimensional electron gas," Phys. Rev. B 38, 10798-10811 (1988).

255. R. Gottinger, a Gold, G. Abstreiter, G. Weimann, and W. Schlapp, "Interface Roughness Scattering and Electron Mobilities in Thin GaAs Quantum Wells," Europhys. Lett. 6, 183-188 (2007).

256. S. S. P. Parkin, "Origin of enhanced magnetoresistance of magnetic multilayers: Spin-dependent scattering from magnetic interface states," Phys. Rev. Lett. 71, 1641-1644 (1993).

257. P. Zahn, J. Binder, I. Mertig, R. Zeller, and P. H. Dederichs, "Origin of giant magnetoresistance: Bulk or interface scattering," Phys. Rev. Lett. 80, 4309 (1998). 